\title{
How plants balance competitive growth and defence an analysis of virtual plants in dynamic interactions
}

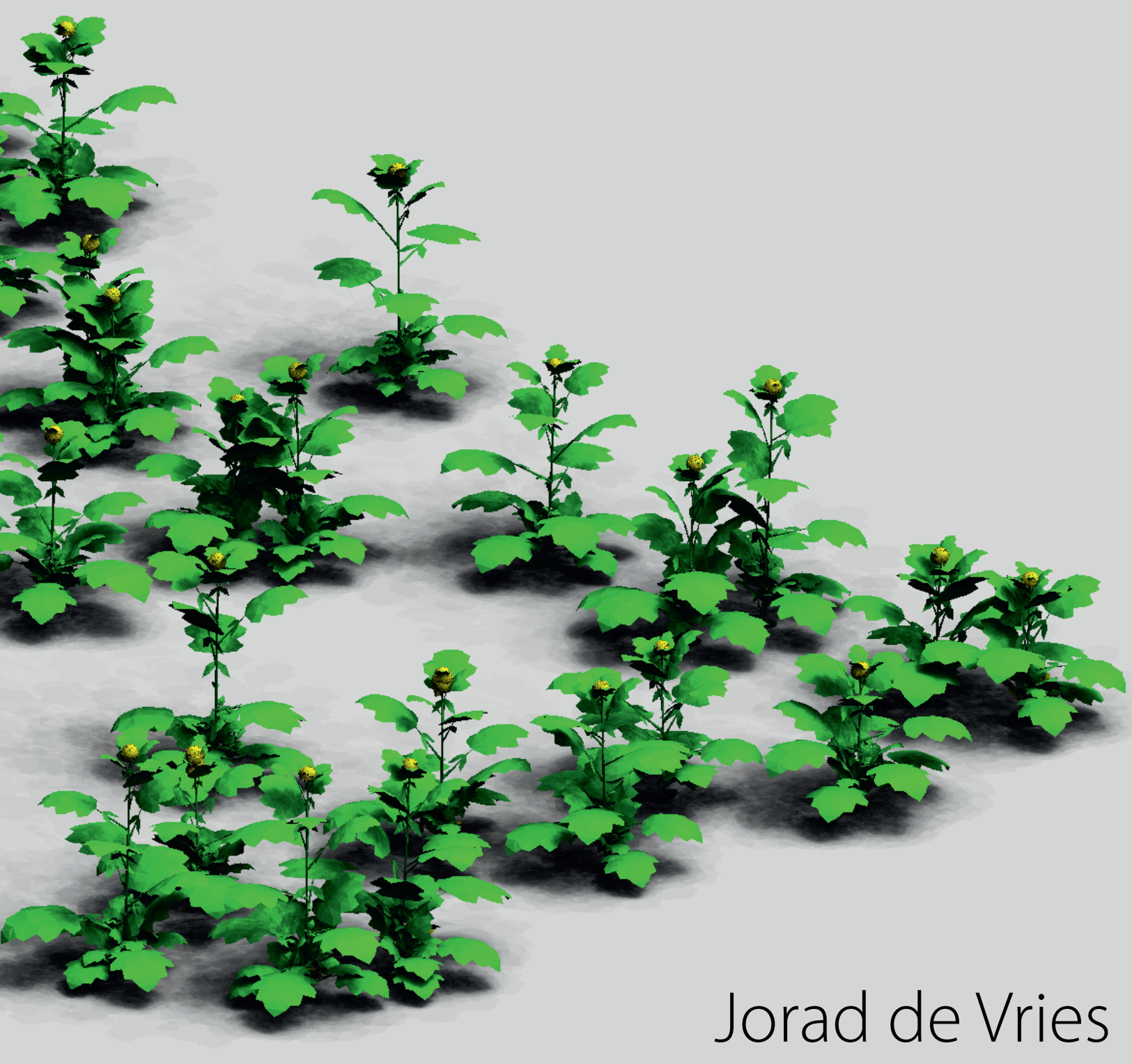



How plants balance competitive growth and defence: an analysis of virtual plants in dynamic interactions

Jorad de Vries 


\section{Thesis committee}

\section{Promotors}

Prof. Dr Marcel Dicke

Professor of Entomology

Wageningen University \& Research

Prof. Dr Niels P.R. Anten

Professor of Crop and Weed Ecology

Wageningen University \& Research

\section{Co-promotors}

Dr. Erik H. Poelman

Associate Professor, Laboratory of Entomology

Wageningen University \& Research

Dr. Jochem B. Evers

Associate Professor, Centre for Crop Systems Analysis

Wageningen University \& Research

\section{Other members}

Prof. Dr Gerlinde B. De Deyn, Wageningen University \& Research

Dr Frank J. Sterck, Wageningen University \& Research

Prof. Dr Ronald Pierik, Utrecht University

Dr Tobias Züst, University of Bern, Switzerland

This research was conducted under the auspices of the C.T. de Wit Graduate School for Production Ecology and Resource Conservation 


\title{
How plants balance competitive growth and defence: an analysis of virtual plants in dynamic interactions
}

\author{
Jorad de Vries
}

\section{Thesis}

submitted in fulfilment of the requirements for the degree of doctor

at Wageningen University

by the authority of the Rector Magnificus,

Prof. Dr A.P.J. Mol,

in the presence of the

Thesis Committee appointed by the Academic Board

to be defended in public

on Friday 8 February 2019

at 1.30 p.m. in the Aula. 
Jorad de Vries

How plants balance competitive growth and defence:

an analysis of virtual plants in dynamic interactions,

162 pages.

PhD thesis, Wageningen University, Wageningen, the Netherlands (2019)

With references, with summaries in English and Dutch

ISBN: 978-94-6343-392-1

DOI: https://doi.org/10.18174/466162 


\section{Table of Content}

Chapter $1 \quad$ General introduction

Chapter 2 Dynamic plant-plant-herbivore interactions govern plant growth- 9 defence integration

Chapter 3 Elucidating the interaction between light competition and herbivore feeding patterns using functional-structural plant modelling

Chapter 4 Ecological interactions shape the adaptive value of plant defence: 57 herbivore attack versus competition for light

Chapter 5 The ecological significance of light quality in optimising plant 75 defence

Chapter 6 Simulation of optimal defence against herbivores under resource 101 limitation and competition using an evolutionary functionalstructural plant model

Chapter $7 \quad$ General discussion

References

English summary

Nederlandse samenvatting

Acknowledgements

Curriculum vitae

Publications

PE\&RC Training and Education Statement 



\section{Chapter 1}

General introduction 
Plants grow in dynamic environments where their fitness is determined by biotic and abiotic conditions. These environments house complex webs of interactions in which plants must compete with neighbours for limited resources, while having to defend against a multitude of heterotrophic organisms such as insect herbivores. Plants have evolved adaptive responses to these stressors, allowing them to perceive and respond to environmental cues related to neighbour presence (Ballaré et al., 1990; Aphalo et al., 1999; Pierik et al., 2013) and herbivore attack (Heil \& Ton, 2008; Howe \& Jander, 2008; Pierik et al., 2014), which allow plants to maximise fitness in variable and dynamic environments (Sultan, 2000). The general consensus is that these plastic responses to signals related to neighbour presence and (future) herbivore attack are constrained by resource availability and compete for the limited pool of an individual's internal resources, resulting in trade-offs between the ability of a plant to compete and to defend (Herms \& Mattson, 1992; Ballaré \& Pierik, 2017; Züst \& Agrawal, 2017). The optimal expression of traits related to competitive growth and defence is not only dependent on environmental conditions such as nutrient availability, plant density and herbivore density, but also on the strategies adopted by herbivores and other plants in the environment (McNickle \& Dybzinski, 2013). This makes the balance between the expression of growth and defence traits highly dependent on ecological interactions with the plant and herbivore communities and therefore highly dynamic in both space and time (Heil, 2002; Strauss et al., 2002; Cipollini et al., 2014). However, it remains unclear how these ecological interactions shape the optimal balance between the expression of traits related to competitive growth and defence.

The aim of this thesis is to elucidate how ecological interactions drive selection for the optimal balance between the acquisition and protection of resources (Fig. 1). To reach this objective, I considered three levels in spatial and temporal integration; a physiological, ecological and evolutionary perspective. First, the physiological responses to competition and herbivory will be quantified and the impact of these responses on plant form and function will be assessed. Second, the impact of these physiological responses to the performance of a plant will be assessed in a dynamic environment, where the plant is influenced by herbivores and other plants and vice-versa. Finally, it is important to consider how these ecological interactions shape the adaptive value of competitive growth and defence in an evolutionary perspective. In this thesis I consider the aforementioned levels of integration by combining field experiments, a more detailed physiological functional-structural plant (FSP) model and an evolutionary FSP model that combines a simplified plant physiology with an evolutionary algorithm. In this general introduction I will introduce the balance between competitive growth and defence from 
a physiological, ecological and evolutionary perspective and introduce FSP modelling as the main tool to reach the objective of this thesis.

\section{The physiology of competitive growth and defence}

Plants respond to neighbour presence with a suite of morphological changes that function to maximise light capture in a competitive environment and includes upward movement of leaves, enhanced petiole and internode elongation, and reduced branching. This collection of morphological changes is named the shade avoidance response (SAS) (Fraser et al., 2016; Ballaré \& Pierik, 2017) and is mediated by a multitude of mechanical (de Wit et al., 2012), volatile (Pierik et al., 2004a; Pierik et al., 2004b) and light signals (Ballaré et al., 1990; Zhang et al., 2011; Keuskamp et al., 2012; Ulm \& Jenkins, 2015), of which the ratio of red to far-red in the light spectrum (R:FR) is one of the best documented. The R:FR ratio is a good predictor of impending competition, as plant tissues readily absorb red light for photosynthesis, while they reflect and transmit the majority of far-red light (Ballaré et al., 1990). Plants register a decrease in the R:FR ratio using phytochromes (Ballaré, 2009; Pierik \& de Wit, 2014) and are known to have specific sites of perception tied to a specific response, such as the leaf tip for a hyponastic response (Pantazopoulou et al., 2017) and stems for an elongation response (Ballaré et al., 1989; Ballaré et al., 1990). Additionally, a low R:FR ratio is known to negatively affect direct resistance to herbivores and pathogens by reducing plant sensitivity to jasmonic acid (JA), one of the essential phytohormones in the regulation of plant defence (Moreno et al., 2009; Cerrudo et al., 2012; de Wit et al., 2013; Campos et al., 2016). This linkage of R:FR and defence hypothetically allows the plant to express different levels of defence in different environments, favouring competitive growth over defence in competitive environments with a high plant density (Ballaré, 2014). Additionally, this linkage allows the plant to concentrate its defences on organs that contribute most to plant fitness through a favourable positioning with respect to the light gradient in the canopy (Izaguirre et al., 2013). Plants are known to induce a stronger defence response in young leaves (Koricheva \& Barton, 2012), which are also favoured in the allocation of nitrogen to maximise photosynthetic gain with respect to the light gradient (Hirose et al., 1987; Anten et al., 1995; Hikosaka et al., 2016). However, this increased investment of limited nutrients also makes these young leaves prime targets for the herbivores attacking the plant (Feeny, 1976; Cates, 1980; Schoonhoven et al., 2005). Understanding how the linkage between competitive growth and defence sits within the framework of other physiological mechanisms is vital to understand how competitive growth and defence affect plant performance and competitive ability. 


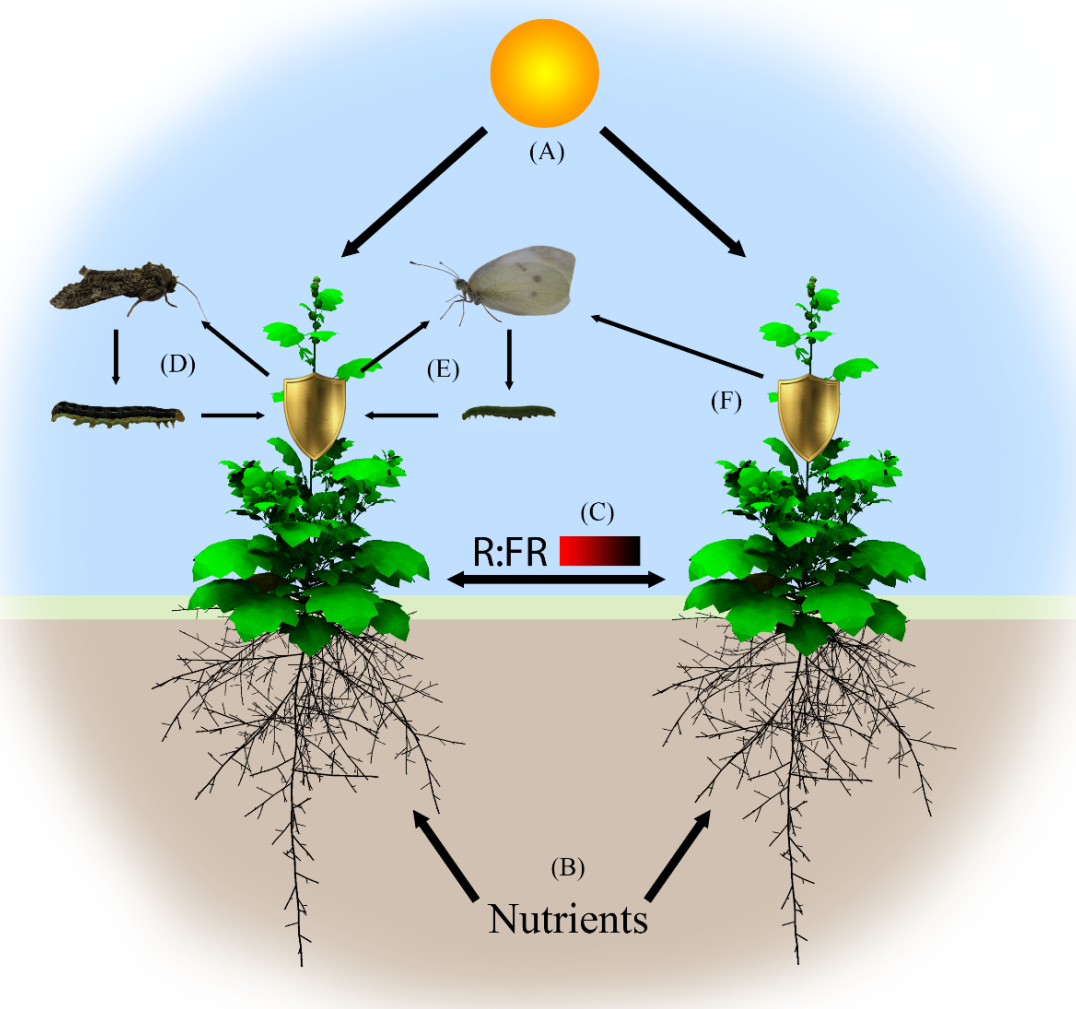

Fig 1. Plants compete for resources that are essential for maintaining growth and fitness, such as light (A) and nutrients (B). To optimise their competiveness, plants alter their morphology and physiology when neighbours are present, through the perception of neighbour detection cues such as the R:FR ratio (C). These cues also downregulate the plants ability to defend itself against insect herbivores, that respond to and induce plant defence expression (D). The induction of plant defence by an attacker influences how subsequent attackers perceive and colonise the plant (E). Additionally, the colonisation of herbivores is not only dependent on the plant defence expression, but also on the defence expression of its neighbours (F).

\section{The ecology of competitive growth and defence}

Changes in plant growth and defence expression are often related to changes in biotic or abiotic environmental conditions (Sultan, 2000), which allows plants to balance the costs and benefits of these physiological mechanisms in their ecological context. It is apparent that plant defence mechanisms carry substantial costs, including the machinery for the synthesis, modification, transport, maintenance and storage of secondary metabolites (Gershenzon, 1994; Bekaert et al., 2012). These direct costs do not necessarily result in a fitness loss and are potentially only 
relevant under certain ecological conditions such as resource limitation, competition for resources or the presence of herbivores and pathogens (Heil, 2002; Koricheva, 2002; Strauss et al., 2002; Dicke \& Hilker, 2003; Cipollini et al., 2014). A reduction in plant competitiveness through an investment in defence can have profound consequences for plant fitness, as competition between plants for a limited resource can be highly asymmetric. Asymmetric competition means that stronger competitors take a disproportionate share of resources and thereby achieve a disproportionately high fitness, which is exemplified in the competition for light (Weiner, 1990; Freckleton \& Watkinson, 2001).

Another important ecological interaction to be considered is the effect that plant defence expression has on the herbivore community and vice-versa. Different herbivore species are known to induce different defence responses in their host plant, governed by their method of feeding and their level of specialisation (Glazebrook, 2005; Schoonhoven et al., 2005). The herbivore community can be roughly classified into generalist and specialist species based on herbivore susceptibility to taxon-specific secondary metabolites (Bennett \& Wallsgrove, 1994). Generalist herbivores can feed from a wide variety of host plants but are not particularly resistant to any one secondary metabolite, causing them to avoid host plants with high levels of secondary metabolites (Feeny, 1976). Specialist herbivores have adapted their metabolism to tolerate a particular taxon's secondary metabolite, but are restricted in their host selection to plants of that particular taxon (Feeny, 1976). Additionally, specialist herbivores prefer host plants with high levels of secondary metabolites as these provide a reliable host-identification cue (Jaenike, 1990; Wheat et al., 2007; Poelman et al., 2008a). This difference in resistance to secondary metabolites allows specialist herbivores to feed from highly nutritious but well defended young leaves whereas generalist herbivores tend to feed from older and less defended leaves (Cates, 1980; Schoonhoven et al., 2005). Plant defence expression thereby mediates the distribution of herbivores within and between plants and can shape the composition of the herbivore community experienced by the plant (Whitham et al., 2006; Whitham et al., 2012). Induction of plant defence by a particular herbivore affects the colonisation of subsequent herbivores, meaning that the individuals in the herbivore community also shape that community as they interact with other herbivores through their effects on the plant (Poelman et al., 2008a; Poelman \& Dicke, 2014; Stam et al., 2014). This can lead to particular herbivore species playing a disproportionately large role in determining plant fitness through their effect on the herbivore community experienced by the plant (Poelman \& Kessler, 2016). To understand the balance between competitive growth and defence it is vital to place these physiological 
mechanisms in their ecological context and quantify the relative importance of interactions within the plant and insect communities, as well as interactions between the two.

\section{The evolution of competitive growth and defence}

Game theoretical studies of density- and frequency-dependent interactions between plants have shown that plant fitness is often not determined by the absolute value of a trait, but by its value relative to the trait values of neighbouring plants (McNickle \& Dybzinski, 2013). This can lead to what is called a tragedy of the commons (Falster \& Westoby, 2003; McNickle \& Dybzinski, 2013), where the expression of a trait is driven away from the population-level optimum to maximise the fitness of the individual, to the detriment of the community as a whole. These tragedies are prevalent in environments where plants compete for limited resources, and overexpression of traits related to resource acquisition has been shown both aboveground (King, 1990; Oksanen, 1990; Zhang et al., 1999; Anten, 2002; Falster \& Westoby, 2003; Dybzinski et al., 2011) and belowground (Givnish, 1995; Gersani et al., 2001; O'Brien et al., 2005; O'Brien et al., 2007; Dybzinski et al., 2011; but see Chen et al., 2015). The overexpression of traits related to resource acquisition may go at the expense of other functions such as defence, leading to the aforementioned trade-offs between competitive growth and defence (Züst \& Agrawal, 2017). To understand the balance between competitive growth and defence, it is imperative to consider the evolutionary dynamics that have led to the linkage between these mechanisms that we observe in plants today. Therefore, it is crucial to understand when natural selection favours traits that promote the acquisition or protection of resources and how this can lead to tragedies of the commons or trade-offs between growth and defence.

\section{Functional-structural plant modelling of competitive growth and defence}

While research in recent years has elucidated much of the physiological mechanisms that link competitive growth and defence (Ballaré, 2014; Mazza \& Ballaré, 2015; Ballaré \& Pierik, 2017), the ecological implications and evolutionary history of this linkage remains largely unexplored. Experimentally studying growth-defence integration on ecological and evolutionary scales is exceedingly challenging, which provides opportunities for complex modelling approaches that can place physiological mechanisms in an ecological and evolutionary context. FSP models simulate three dimensional (3D) plant canopies, where individual plants or plant organs are explicitly represented with their own structure and orientation in the 3D scene (Vos et al., 2010; Evers et al., 2018). These models can range from purely descriptive representations of plant architecture (Zhu et al., 2015) to highly dynamic and 
mechanistic models where plant form and function depend on the interactions between plant physiological processes and the environment (Evers \& Bastiaans, 2016; Bongers et al., 2018). These structural traits and physiological processes can then be scaled to whole-plant phenotype, performance and ultimately plant fitness. Aboveground interactions between plants are generally modelled through the light environment, simulating absorbed photosynthetically active radiation (PAR) and the perceived R:FR ratio of individual leaves based on canopy structure as a result of plant architecture. The R:FR ratio can be used to calculate shade avoidance and defence responses, mediating changes in plant architecture and subsequent changes in light conditions. Belowground, plants compete for resources such as nitrogen, which is needed by the plant to grow and photosynthesise. The acquisition of above- and belowground resources can be used to calculate rates of photosynthesis and subsequent plant growth through source-sink dynamics. This foundation of plant structure and functional mechanisms make FSP models a strong tool to study the physiological, ecological and evolutionary functioning of growth-defence integration in plants, providing crucial insights and guiding future experimental work.

\section{This thesis}

In this thesis, I will analyse how ecological interactions shape the optimal balance between competitive growth and defence in a dynamic environment. I will use the annual weed Brassica nigra as a model plant, as it occurs in a wide range of plant densities (Lankau \& Strauss, 2008), exhibits a strong morphological response to competition, resulting in distinctly different plant phenotypes (Ballaré \& Pierik, 2017; Pantazopoulou et al., 2017), and is widely used as a model plant in the field of plant-herbivore interactions (van Dam \& Raaijmakers, 2006; Lankau \& Strauss, 2008; Broekgaarden et al., 2011). The low-density phenotype of B. nigra is highly branched with large, flat leaves while the high-density phenotype is a single stem with small, upwards oriented leaves. Additionally, B. nigra occurring in natural settings host a wide variety of herbivorous insects that include sap-sucking and chewing herbivores of different levels of specialisation (Stam et al., 2014).

In Chapter 2, I outline my view on the importance of considering dynamic interactions within and between the plant and insect communities when looking at the trade-off between competitive growth and defence. I propose the use of FSP modelling as a tool to simulate these interactions from physiological to ecological scales and impact the trade-off between competitive growth and defence. 
Chapter 3 presents an FSP model of B. nigra that combines mechanistic simulation of plant growth and shade avoidance responses, which were parameterised and validated using field experiments. The simulated plants are infested with an agent-based representation of chewing herbivores that impact the dynamic feedback between plant architecture and the light environment. With this model I analyse the impact of different herbivore feeding locations related to herbivore specialisation on plant fitness in a dynamic and competitive environment.

In Chapter 4, I focus on the costs and benefits of plant defence in a dynamic environment, expanding on the physiological FSP model of B. nigra presented in chapter 3. With this model I analyse how direct and indirect costs that are imposed through ecological interactions impact plant fitness. Additionally, I analyse how the plant benefits from an investment in plant defence through either a reduction in herbivore damage or a dispersion of herbivore feeding within the plant.

In Chapter 5, I focus on the ecological function of the R:FR mediation of plant defence in an evolutionary perspective. Expanding on the $B$. nigra FSP model presented in chapter 4, I analyse whether the R:FR mediation of plant defence functions to optimise whole-plant defence expression over different densities, or to optimise the distribution of defence within the plant. I perform this analysis from the perspectives of simple and competitive optimisation (e.g. in mono-stands and mixtures, respectively), and discuss the consequences of de-coupling R:FR mediation of plant defence in crop plants.

Chapter 6 presents a new FSP model linked to an evolutionary algorithm that simulates how a population of plants evolves over multiple generations as the plants compete for resources and are under attack by insect herbivores. With this model I analyse how biomass allocation patterns relate to plant density, nitrogen availability and herbivore pressure, and how these factors impact the optimal balance between the acquisition and protection of resources.

In the general discussion (Chapter 7), I discuss the conclusions of this study in relation to the main objective, emphasising the importance of considering dynamic interactions between the plant and the biotic and abiotic factors in the environment. I discuss future perspectives in the development of FSP models and how these models may be utilised in research that scales from physiological mechanisms to eco-evolutionary dynamics. Finally, I will present some additional simulations conducted with the evolutionary FSP model presented in chapter 6 to illustrate some of the ideas presented in this discussion. 


\title{
Chapter 2
}

\section{Dynamic plant-plant-herbivore interactions govern plant growth-defence integration}

\author{
Jorad de Vries ${ }^{1,2}$, Jochem B. Evers ${ }^{2}$ and Erik H. Poelman ${ }^{1}$ \\ ${ }^{1}$ Wageningen University, Laboratory of Entomology, Wageningen, The Netherlands \\ ${ }^{2}$ Wageningen University, Centre for Crop System Analysis, Wageningen, The Netherlands
}




\title{
Chapter 2
}

\begin{abstract}
Plants down-regulate their defences against insect herbivores upon impending competition for light. This has long been considered a resource trade-off, but recent advances in plant physiology and ecology suggest this mechanism is more complex. Here we propose that to understand why plans regulate and balance growth and defence, the complex dynamics in plant-plant competition and plant-herbivore interactions needs to be considered. Induced growth-defence responses affect plant competition and herbivore colonisation in space and time, which has consequences for the adaptive value of these responses. Assessing these complex interactions strongly benefits from advanced modelling tools that can model multi-trophic interactions in space and time. Such an exercise will allow a critical reevaluation why and how plants integrate defence and competition for light.
\end{abstract}




\section{Growth and defence in a dynamic context}

To maximise its fitness a plant relies on the ability to perceive and respond to its surroundings, which are highly dynamic and can pose a multitude of threats as well as opportunities. In dense vegetation plants have to both compete with neighbour plants for resources and defend themselves against herbivores and pathogens. These constraints force plants to balance their investment in growth and defence, which has been widely described as growth-defence tradeoffs (see Glossary). One particular example of such a trade-off that is prevalent in weedy plants that grow in direct sunlight of open vegetation, is a plant's response to downregulate its defences when competing for light. This mechanism was first described as a resource trade-off between the plant growth response to light competition, the shade avoidance syndrome (SAS) (Franklin, 2008; Pierik \& de Wit, 2014; Fraser et al., 2016)(see Glossary), and defence (Herms $\&$ Mattson, 1992). However, recent advances have shown how light signals, most notably the ratio of red to far-red light (R:FR, see Glossary), directly mediate plant defences (de Wit et al., 2013; Ballaré, 2014; Campos et al., 2016) (see Box 1). Although the downregulation of defences by R:FR seems to be universal for a broad range of plant species under attack by different types of attackers from pathogens to insects (Moreno et al., 2009; Cerrudo et al., 2012; Izaguirre et al., 2013; Kegge et al., 2015), the effect of this response on the dynamics of plant and attacker communities can be very specific and exert strong feedback on the adaptive value of this response. However, the downregulation of defences by R:FR is still mostly considered in the generic and static context of resource trade-offs (Ballaré, 2014), rather than in the context of highly dynamic plant and attacker communities that may impose ecological costs to a growth-defence response. The consequences of these species-specific dynamics on the interaction between mechanisms of growth and defence are still largely unknown, but may encompass an important factor in driving plant physiological adaptations in regulation of growth and defence. In this opinion paper we consider the downregulation of defences by R:FR in the context of dynamic plant-plant-herbivore interactions.

Recent advances have shown that dynamics in herbivore-plant interactions can have a profound impact on the adaptive value of inducible plant defences because of feedback loops between induced defence and insect community assembly (Poelman \& Kessler, 2016). Combining these advances in the fields of plant physiology and insect community dynamics will change the way we view the adaptive value of plant defences in a competitive environment, making it timely to question the ecological function of the physiological linkage between shade 


\section{Box 2.1. Plant defences and light}

Plants defend themselves against herbivore attack by producing (taxon specific) secondary metabolites that hinder herbivore growth. Upon herbivore damage defences are induced by both the mechanical wounding (Berger et al., 1995) as well as the chemical signal from the insects saliva (Howe \& Jander, 2008). Plant defences are mediated by a hormonal backbone primarily consisting of the hormones Jasmonic acid (JA), Salicylic acid (SA) and Ethylene (ET) (Pieterse et al., 2012), allowing the plant to tailor its defences to different types of attackers (Ballaré, 2011; Thaler et al., 2012). Plant defences and plant responses to light are physiologically linked: defences can be negatively or positively mediated by light signals, depending on the information of the light signal (Figure I). Defences are negatively regulated by R:FR, leading to negative regulation of defences by simultaneously desensitising the plant for JA and SA (de Wit et al., 2013). Defences are positively regulated by light signals that indicate a favourable light environment: PAR and UV-B radiation (Ballaré, 2014). These signals are known to induce changes in leaf morphology to increase light capture, such as increased leaf thickness, which increases resistance to chewing herbivores (Schoonhoven et al., 2005). Additionally, high light capture increases the rate of photosynthesis and subsequently the level of soluble sugars in the leaf, which also acts as a positive mediator of defences (Berger et al., 1995). The amplified levels of photosynthesis require an increase in transpiration, which leads to an increased influx of xylem-transported cytokinins. These cytokinins are an important growth hormone that positively mediates plant defences as well as primary cell growth (Giron et al., 2013; Ballaré, 2014). These positive regulations of defences are in parallel with an upregulation of photosynthesis and nitrogen allocation, also mediated by light signals (Niinemets \& Anten, 2009). However, a positive mediation of defences by light signals requires a working JA signalling pathway. The desensitization of plant tissues to JA by low R:FR will supress the light mediated upregulation of defences, denoting that the signal for impending competition overrules currently favourable light conditions (Ballaré, 2014).

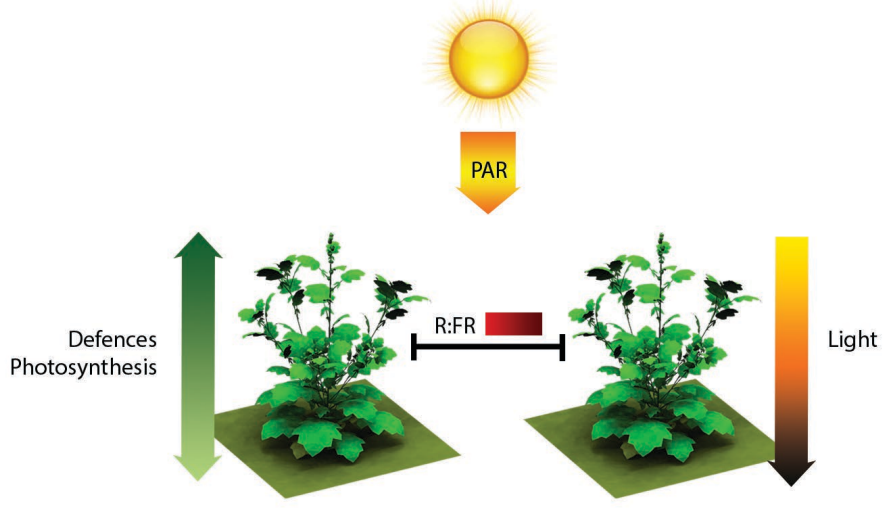

Figure 2.I. The allocation of both defences and photosynthetic capacity follows the light gradient in the canopy, mediated by transpiration driven transport of cytokinins. The leaves in the top of the canopy will intercept more light and subsequently have a higher transpiration rate to sustain a higher rate of photosynthesis. This increased transpiration increases the influx of cytokinins into leaves which will enhance growth as well as elevate the level of defence. Both defences and photosynthetic capacity are negatively mediated by the ratio of red to far-red light (R:FR), a signals that denotes competition. 


\section{Box 2.2. Glossary}

Functional Structural Plant (FSP) Modelling: This 3D modelling approach is an established method that describes plant architectural development (i.e. production of new organs at a certain rate with certain geometrical properties like orientation), assimilate production and plant growth (Vos et al., 2010).

Growth-defence trade-off: Describe trade-offs between a plants ability to grow and defend against attackers, often in the context of constitutive plant strategies. The dilemma of plants (Herms \& Mattson, 1992) was first described as a trade-off between shade avoidance growth and defences. Now we know the down-regulation of plant defences under the influence of competition is directly mediated by a drop in the red to far-red ratio (Moreno et al., 2009; Campos et al., 2016) (see shade avoidance response and R:FR).

Optimal defence theory (ODT): States that plants concentrate the allocation of defences towards those parts where these defences are most beneficial to the plant, e.g. parts of high value or likely to be attacked by herbivores, under the assumption that defences are costly yet beneficial to the plant. (McKey, 1974; Fagerstrom et al., 1987)

R:FR: The ratio of red to far-red light intensities. Plant tissues absorb red light, while they reflect and transmit far-red light. Therefore, the presence of neighbouring plants results in a drop in the ratio between red and far-red light, making it a robust signal for impending competition.

Shade avoidance syndrome (SAS): A set of responses that allows the plant to combat the competition for light in dense canopies. Plants integrate a number of above and belowground cues to detect the presence of neighbours (Pierik et al., 2013; Pierik \& de Wit, 2014; Fraser et al., 2016), of which the red to far-red ratio (see R:FR) in the light spectrum is the most important (Ballaré et al., 1990). Upon neighbour detection the plant will alter branching patterns, angle leaves upwards, promote stem elongation and flower earlier in order to enhance light capture and minimise fitness losses. (reviewed by Franklin (2008))

avoidance and defence. In this opinion paper we therefore argue the need to step away from considering the interaction between shade avoidance growth and defence in a static context of a single ecological setting at a single time point, and start considering complex dynamics of plant-plant-herbivore interactions through space and time. Importantly, the trade-off between growth and defence may play out differently over plant ontogeny and in the context of feedback loops in plant-insect communities. We argue that the first challenge lies in understanding how the regulatory mechanisms of induced defences impact the plant community in a competitive environment. The second challenge lies in understanding how induced defences impact insect community dynamics and how these dynamics feed back to determine plant fitness in a competitive environment (Figure 1.). In this opinion paper we discuss four possible mechanisms that could explain the function of light mediated downregulation of defences from the perspective of plant-herbivore interactions in open vegetation; 1) Downregulation of defences by light is in concordance with optimal defence theory (ODT) (see Glossary), and is a mechanism to focus defences on valuable plant tissues. 2) Feeding patterns of specialist 


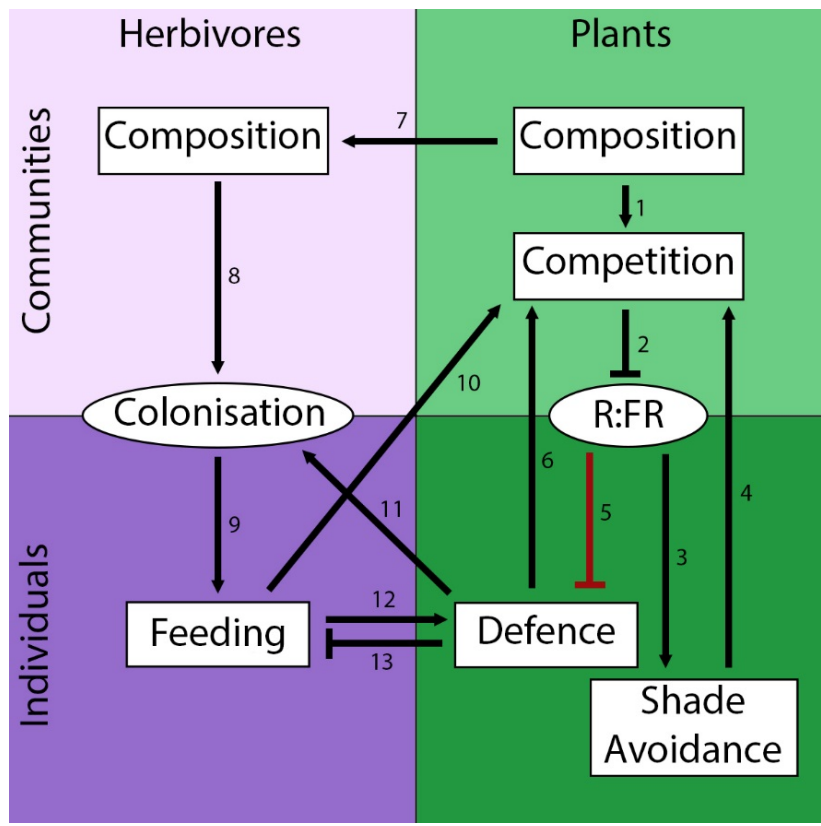

Figure 2.1. A cause-effect diagram of the interactions between plants (green) and herbivores (violet) on the community (light) and individual (dark) scales. The plant community composition in terms of species and density determines the level of competition in the vegetation (1), which translates into an R:FR signal that can be registered by the individual plants (2). This R:FR signal initiates the shade avoidance response (3), which affects the severity and outcome of the competition within the vegetation (4). Additionally, the R:FR signal downregulates plant defences (5), which is the focus of this paper. These defences are induced by damage by plant attackers such as insect herbivores feeding on the plant (12) and act to reduce the damage done by these inducing herbivores (13). Induction of these defences does carry some direct metabolic cost, which affects the plants competitiveness and therefore affects the outcome of competition on the community level (6). These costs (6) are seen as the reason for the downregulation of defences by R:FR (5) to exist when looking at the system from a resource trade-off perspective. However, community processes such as feedback loops or indirect interactions among community members may encompass important costs that are overlooked in a resource-driven trade-off. The damage done by the individual herbivores will remove leaf area and thereby change the outcome of the competition on the vegetation level (10). Moreover, the attacker community will respond to the induced plant phenotype (11). For insect herbivores these responses may be specific for the type of herbivore (8) and modulated by the composition of the plant community surrounding the induced plant (7). We hypothesise that the interactions between plant communities and herbivore communities (7), plant defences and herbivore colonisation (11) and herbivore damage and competition (10) play an important role in regulation of defences in competition (5).

herbivores in combination with the specialists use of plant defences as a host detection cue may have driven selection away from inducible defences to protect valuable tissues. 3) The attraction of a specific herbivore species by changes in plant phenotype due to an earlier infestation may 
have driven selection toward negative regulation of defence when competing for light. 4) Plants could downregulate defence to prevent providing neighbouring plans with information that would induce growth or defence responses, increasing the neighbours competitiveness. We propose to utilize a three-dimensional modelling technique to simulate the temporal and spatial dynamics in plant competition and herbivory. This modelling technique allows us to evaluate the impact and sensitivity of individual parameters in a wide range of complex ecological settings, which reaches beyond experimental feasibility. This approach can assess the value of plant defences in complex and dynamic interactions with the light environment, competition with neighbouring plants and the herbivore community, guiding our experimental approach to validate model predictions. This exercise will lead us to re-evaluate the selective forces impacting on plants in a competitive environment and understanding the role of these forces in balancing shade avoidance growth and defence.

\section{Light drives optimal defence allocation}

In the context of resource trade-offs, Ballaré hypothesised that light signals perceived by a leaf may be indicative of the value of that leaf to the plant (Ballaré, 2014). Due to shading within the vegetation, plants typically have sunlit and shaded leaves where the former are more valuable to the plant in terms of photosynthetic gain. Based on the ODT (McKey, 1974), it could thus be argued that sun-lit leaves should also be better defended. Additionally, horizontal reflectance of low R:FR by neighbour plants indicates a leaf is facing impending competition and may become shaded in the future. Such a leaf may therefore become of low value to the plant when it indeed will be shaded by other leaves and should be less defended, even when the R:FR signal occurs in conjunction with high light conditions (Izaguirre et al., 2013). This integration of light signals allows the plant to allocate its defences along a future light gradient and towards its most valuable organs. Leaves at the top of the canopy are crucial for the plant to stay competitive in the battle for light with neighbouring plants. The penalty for losing these valuable leaves can be disproportionally large, while spending resources on defending a leaf that has little value to the plant is marginally beneficial, especially in a competitive environment. However, research in other study systems with different attackers (molluscs and aphids) and a limited level of competition indicate that plant defence allocation might disagree with ODT in the presence of competition (Lankau \& Kliebenstein, 2009) and that investment in defence might be favoured in interspecific competition (Lankau \& Strauss, 2008). Therefore, we argue the need to consider the downregulation of defence by R:FR in a broader ecological context to investigate the mechanisms conformity with ODT. 


\section{Costs and resource trade-offs}

One of the main assumptions of ODT and resource trade-offs is that the production and maintenance of plant defences carry high costs to the plant. However, considering these costs in their dynamic ecological context offers a different perspective than viewing these costs at a single moment in a static environment. This is exemplified by the asymmetry of light competition; a small difference in competitiveness between two neighbouring plants can lead to a difference in resource gain which amplifies the difference in competitiveness between the two plants. The direct costs of induced plant defences come in two forms. First, the plant invests resources in the synthesis and transport of secondary metabolites to combat the herbivore threat. Second, the plant redistributes valuable resources such as nitrogen in RuBisCo and soluble proteins away from damaged leaves upon herbivore damage, while redistributing secondary metabolites towards these damaged leaves (Nabity et al., 2009; Ullmann-Zeunert et al., 2013). Although a local induction of defences might be costly, it is also beneficial to the plant as it reduces herbivore damage (Baldwin, 1998), and potentially affect plant competitiveness by serving additional functions such as in primary metabolism (Jones et al., 2006; Lankau \& Kliebenstein, 2009). After the initial defence response the plant can mobilize a secondary response in systemic tissues, either by inducing defences or priming for future induction. Priming reduces the costs of defence in the absence of herbivory and allows plants to respond quicker and stronger upon herbivore infestation (Conrath et al., 2006), making priming a very cost effective mechanism in plant defence (van Hulten et al., 2006). The fitness costs of induced defences are highly dependent on the plants dynamic environment, and are hard to quantify because of complexity in the functioning of primary and secondary metabolism and how this interacts with the environment (Neilson et al., 2013). All costs, be it biosynthetic or ecological, eventually wind down to fitness costs that are ecological in nature and should therefore be considered in a dynamic context. We recognise that the balancing of growth and defence is to optimise fitness and that in a dynamic context, even low costs can be detrimental to fitness because of the asymmetry of light competition. However, without clear evidence that the costs of defence outweigh its benefits we argue that to explain the function of the downregulation of defences by R:FR we need to look further than the context of resource trade-offs and ODT and consider a broader ecological context.

\section{Herbivore colonisation at the plant level}

The dynamic processes of induced growth and defence responses affect plant competition and herbivore colonisation in space and time, which has consequences for the feedback between 
plant defences and the herbivore community. The relative size and defence phenotype of a plant in the community determines the likelihood of colonisation by different herbivores. Relatively larger plants may be exposed more to herbivory as they are more conspicuous in the plant community or because herbivores select larger plants with more biomass for egg deposition (Feeny, 1976). Similarly, the defence phenotype determines the apparency of a plant to herbivores and particularly affects the type of herbivore species that colonize the plant. Herbivorous insects can be roughly classified into two types: generalist and specialist species, although herbivore food plant specialisation has a continuous spectrum. Specialist herbivores have adapted their metabolism to be more tolerant to taxon-specific secondary plant metabolites, but require a host of that particular taxon. Therefore, specialist herbivores require reliable cues to identify their host and find these in the volatile and non-volatile host plant specific chemistry. In contrast, generalist species have toxin avoidance mechanisms not particularly resistant to any one secondary metabolite but can feed from a variety of host plants, often avoiding those with high levels of secondary chemistry (Feeny, 1976). As a result, the behavioural preferences of the herbivore reflect their susceptibility to the plants defences: generalists tend to prefer less defended plants for oviposition while specialists tend to prefer more defended plants for oviposition (Poelman et al., 2008a; Stam et al., 2014) (Figure 2). Similarly, generalists have to feed on less defended leaves lower in the canopy while specialists can target young leaves in the top of the canopy, which have a higher nutritional value but are also better defended. While specialists do have a feeding preference for lower-defended plants if given the choice (Kegge et al., 2013), they are capable of feeding on the most nutritional leaves within the plant despite elevated defences and frequently initiate feeding here because of oviposition preferences by the adult. These nutritional leaves are often young leaves at the top of the canopy that are hypothesised to be vital to the plants survival in a competitive environment, suggesting that specialist herbivores pose a possible threat to plant fitness in such an environment. The presence of specialist herbivores has been shown to affect the selective value of defence allocation within the plant, increasing the defence of valuable young leaves (Alba et al., 2012). In dense plant populations, there might be selective advantage for an individual plant in supressing defence as this makes the plant relatively less attractive for oviposition to specialist herbivores, which would instead colonise neighbouring plants. Conversely, for isolated plants this suppression would be less beneficial as there are no neighbouring plants to offer the herbivores an alternative choice. Alternatively, the plant could attempt to reduce its attractiveness to specialist herbivores in a competitive environment, 


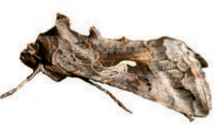

Generalist

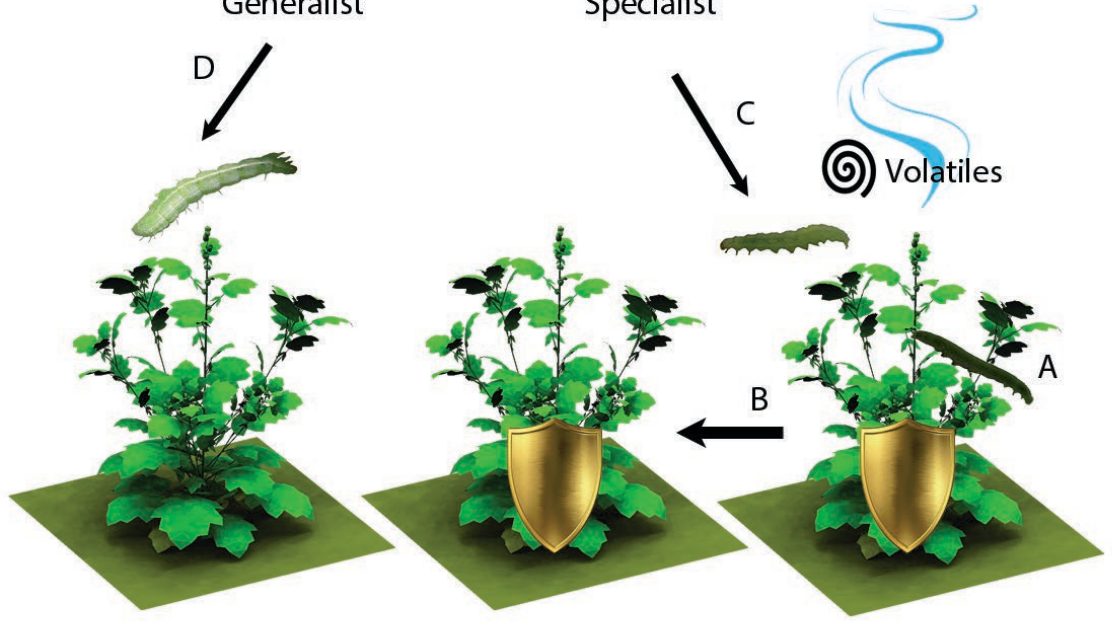

Figure 2.2. Interactions between a plant under herbivore attack (A) and its environment. The attacked plant will induce its defences and send out a volatile signal containing information that can be used by a neighbouring plant to induce its defences (B). The same volatile signal attracts specialist species that will oviposit on these induced plants rather than another, noninduced plant $(\mathrm{C})$, but deter a generalist species, which will rather oviposit on another, noninduced plant (D).

potentially by supressing the defence responses that serve as an important host detection cue for these herbivores (Van Der Meijden, 1996; Poelman \& Kessler, 2016).

\section{Plant defence and the insect community composition}

Plant defences not only drive the spatial distribution of herbivores within the plant, but also determine herbivore distribution dynamics on a larger spatial scale. Induced defences in response to herbivory increase plant resistance to the current attacker, but also change the likelihood that the plant is attacked by subsequent attackers (Viswanathan et al., 2005; Bukovinszky et al., 2010; Poelman et al., 2010; Tack \& Dicke, 2013). The effect of this induced response on the likelihood of subsequent infestation varies with the herbivore inducer as the plant response may be specific to the attacker (Van Zandt \& Agrawal, 2004). Therefore, each herbivore that colonises a plant has a unique effect on the composition of the insect community on the host plant through the induced plant phenotype (Poelman, 2015). The impact of the 
plant's phenotype in terms of growth and defence on the distribution and composition of the insect community feeds back to the host plant to drive selection on plant traits. Therefore, the way plant defences interact with the insect community can pose an indirect cost on induced plant defences. Certain herbivore species might have a more profound effect on the composition of the insect community than other herbivores. These species can exert a positive or negative selection pressure on plant defences through their indirect plant-mediated effect on the rest of the insect community (Poelman \& Kessler, 2016). This concept shows that the information component of plant defences goes beyond their defensive function and has been recognised to be of great ecological significance in shaping both the plant and insect community (Kessler, 2015). This information based perspective suggests selection on plant defences is driven by complex and dynamic interactions between the plant and insect communities. These herbivoreplant-herbivore interactions can be regarded as an additional, ecological, cost to plant defences that can select for or against induced plant defences (McArt et al., 2013) and are thus incorporated in trade-offs between growth and defence.

\section{Growth-defence interactions in an ecological perspective}

Shade avoidance and plant defences have evolved in a highly dynamic context and impact a multitude of species and trophic levels over time and space. It has been suggested that dynamics in both the plant and insect communities determine the selective value of plant defences (Agrawal et al., 2006; Lankau \& Strauss, 2008). In that dynamic context we should consider the defensive function of traits, but also their functioning as competitive traits (Jones et al., 2006; Lankau \& Kliebenstein, 2009) or as a source of information (Kessler, 2015). This information can offer plants a means of defence when it primes systemic tissues or attracts parasitoids. However, the information can also be an important mediator of the insect community composition, and can be used by neighbouring plants to upregulate their defences before they themselves become the victim of herbivore attack (Baldwin \& Schultz, 1983; Heil $\&$ Ton, 2008) (Figure 2). This means of communication between plants will impact herbivore distribution patterns, which can play an important role in the evolution of induced plant defences (Anderson et al., 2015). Competition drives selection towards traits that improve resource acquisition or use efficiency, but also makes plants more susceptible to herbivore damage as the plants ability to compensate damage declines. Additionally, the attraction of specialist herbivores can increase as the density of host plants increases (Schoonhoven et al., 2005). The added pressure of competition makes the information function of induced plant defences more important to plant fitness, and depending on the receiver and its response to the 
information there could be selection for or against induced defences. We expect the informative role of plant defences to have played a key role in the evolution of plant defences, and be a plausible foundation for its down regulation by R:FR.

\section{Suggested approach; 3D plant modelling}

The questions that arise from this complex system cannot be solved with experiments alone, but require the support of novel modelling approaches. We propose the use of functionalstructural plant (FSP) modelling (Vos et al., 2010) (see Glossary) because it allows us to simulate the plant plasticity and spatial heterogeneity that drive plant-plant-herbivore interactions. One of the main strengths of this method is its ability to model the interaction between the light environment and plant morphology (Evers et al., 2011; Bongers et al., 2014). FSP models can simulate the dynamics in plant-plant and plant-herbivore interactions as emergent model behaviour that arises from the interaction between three major mechanistic components. First, light has to be the driving force behind plant morphology and growth, including mechanisms of photosynthesis and shade avoidance (Bongers et al., 2014). Second, to model the relationship between plant growth and defence plant defences need to be incorporated within the FSP modelling framework, simulating its costs, benefits and physiological mediation by light signals. Third, we have to simulate herbivores and their interaction with plant morphology and physiology. The behaviour of the simulated herbivores can be linked with plant phenotypical traits such as leaf age and defensive status. In turn, the herbivores will alter plant functioning at the organ level by interfering with source-sink dynamics of the simulated plants (Anten \& Pierik, 2010), change the light environment through leaf area removal and induce plant defences. This allows us to assess how competition induced differences in plant architecture and leaf physiology and their impact on susceptibility to different types of herbivores (Figure 3.), for example an increased susceptibility to specialist herbivores in a competitive environment. The next step would be to simulate different herbivore and plant communities composed of herbivores with different levels of specialisation and plants with different defence strategies. These models allow us to design virtual plants that integrate growth and defence in different ways and assess their performance in a wide range of ecological settings in a way that experiments cannot. For instance, we can simulate a plant where defences are not regulated by light or where a R:FR signal upregulates rather than downregulates defences. Exercises such as this allow us to generate hypotheses and identify possible ecological drivers that have selected for the downregulation of defences by R:FR. These hypotheses and drivers can guide future experimental work and eventually lead us to reshape the current 


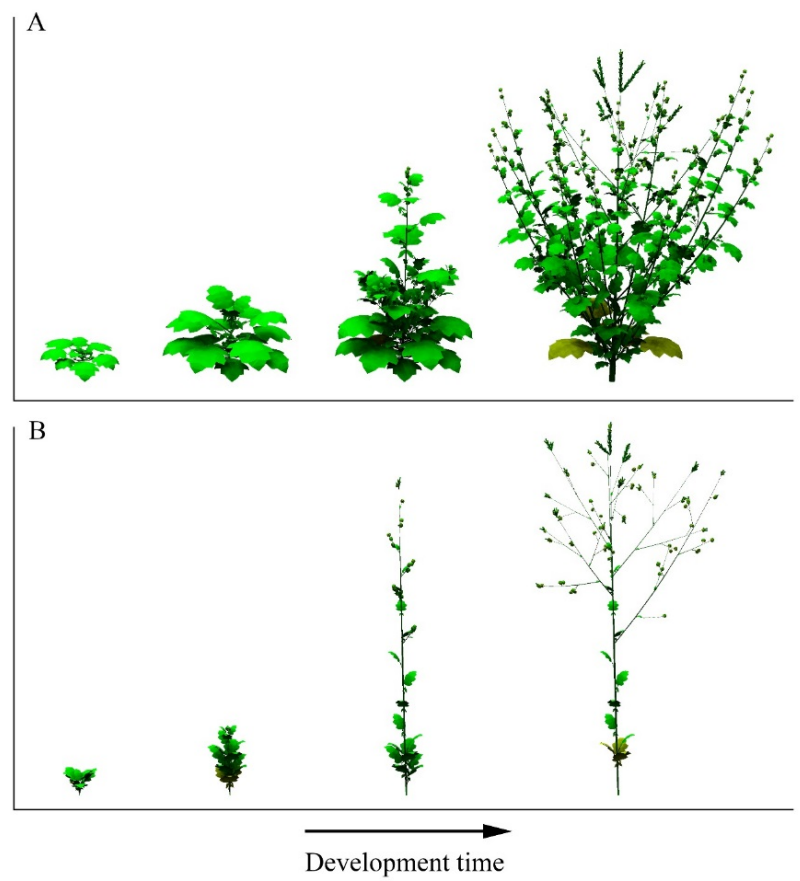

Figure 2.3. Visual output of a mechanistic FSP model of Brassica nigra (de Vries et al., $2018 \mathrm{~b}$ ) showing architectural development over time of a simulated plant growing in a low (A) and a high (B) plant density. These plants will be differentially affected by simulated herbivore damage because of the differences in plant architecture, leaf area and potential seed set in combination with differences in light availability due to competition.

paradigm on shade avoidance growth and defence. FSP modelling complementing experimental approaches is therefore an invaluable tool to help elucidate the origin of the growth-defence trade-off where experiments or other modelling approaches fall short.

\section{Concluding remarks}

When assessing the downregulation of defences by R:FR it is important to consider growth and defence in the entire range of dynamic interactions, rather than the static context of resource trade-offs. We hypothesise that the downregulation of defences by R:FR is not a mechanism to counteract a resource trade-off, but is the result of selection against induced defences due to the interaction between plant competition and insect community dynamics. Because of the difficulty of testing these hypotheses experimentally we propose FSP modelling as the optimal tool to investigate all levels of growth-defence interactions: from small scale plant physiology and insect behaviour to interactions and implications on an ecological scale. In our argumentation, we have come up with alternative hypotheses for down regulation of defences 


\section{Chapter 2}

by R:FR in the growth-defence trade-off for weedy plants that are exposed to direct sunlight. FSP models may allow to manipulate the light environment as well as functioning of R:FR signalling to further predict adaptations to optimize growth-defence responses in other plant species, such as understory weedy plants and woody plants. Future advances that consider growth and defence in a dynamic environment will shed new light on the question why the growth-defence trade-off exists in plants (see outstanding questions). This is a fundamental question in the field of ecology that can be applied to enhance crop resistance to herbivores though breeding or management practices. Understanding how growth and defence are physiologically and ecologically entwined would allow to lift the compromised levels of resistance in dense agricultural cropping systems.

\section{Acknowledgements}

We thank Marcel Dicke, Niels Anten and three anonymous reviewers for constructive comments on an earlier version of the manuscript. This work was supported by the Netherlands Organization for Scientific Research (NWO) (project number: 823.01.004). 


\title{
Chapter 3
}

\section{Elucidating the interaction between light competition and herbivore feeding patterns using functional-structural plant modelling}

\author{
Jorad de Vries ${ }^{1,2}$, Erik H. Poelman ${ }^{1}$, Niels P.R. Anten² and Jochem B. Evers ${ }^{2}$ \\ ${ }^{1}$ Wageningen University, Laboratory of Entomology, Wageningen, The Netherlands \\ ${ }^{2}$ Wageningen University, Centre for Crop System Analysis, Wageningen, The Netherlands
}




\title{
Chapter 3
}

\begin{abstract}

\section{Background and Aims}

Plants usually compete with neighbouring plants for resources such as light as well as defend themselves against herbivorous insects. This requires investment of limiting resources, resulting in optimal resource distribution patterns and trade-offs between growth and defence related traits. A plant's competitive success is determined by the spatial distribution of its resources in the canopy. The spatial distribution of herbivory in the canopy in turn differs between herbivore species as the level of herbivore specialisation determines their response to the distribution of resources and defences in the canopy. Here, we investigated to what extent competition for light affects plant susceptibility to herbivores with different feeding preferences.
\end{abstract}

\section{Methods}

To quantify interactions between herbivory and competition we developed and evaluated a 3D spatially explicit functional-structural plant model for Brassica nigra that mechanistically simulates competition in a dynamic light environment, and also explicitly models leaf area removal by herbivores with different feeding preferences. With this novel approach we can quantitatively explore the extent to which herbivore feeding location and light competition interact in their effect on plant performance.

\section{Key Results}

Our results indicate that there is indeed a strong interaction between levels of plant-plant competition and herbivore feeding preference. When plants did not compete, herbivory had relatively small effects irrespective of feeding preference. Conversely, when plants competed, herbivores with a preference for young leaves had a strong negative effect on the competitiveness and subsequent performance of the plant, whereas herbivores with a preference for old leaves did not.

\section{Conclusions}

Our study predicts how plant susceptibility to herbivory depends on the composition of the herbivore community and the level of plant competition, and highlights the importance of considering the full range of dynamics in plant-plant-herbivore interactions. 


\section{Introduction}

Plants face a multitude of threats over the course of their development and have to balance resource allocation in response to these threats to maximise their fitness. Plants have to compete with neighbouring plants for resources such as light as well as defend against defoliation by herbivorous insects. Both these mechanisms require an investment of limiting resources such as nitrogen and can drive selection towards an optimal distribution of these resources in the canopy (Stockhoff, 1994; Hikosaka et al., 2016). Additionally, due to the potential costs of defences and the strong selective pressure of competition, plants are subjected to trade-offs in the allocation of limiting resources to either growth or defence-related traits (Züst \& Agrawal, 2017). These growth-defence trade-offs are strongly determined by spatiotemporal processes driven by interactions between plants and the plant and insect communities individual plants are part of (de Vries et al., 2017). Plants have developed a highly variable ontogenetic trajectory in growth-defence allocation because plant tolerance to herbivory, the relative costs of defences as well as the selective pressure of competition fluctuate over the course of plant development (Barton \& Boege, 2017). Resource-limiting conditions promote allocation of resources towards organs that are most favourably positioned relative to resource distributions (McKey, 1974). There has been a whole body of literature that has analysed the spatial allocation of resources in relation to maximising canopy photosynthesis, growth and competitive ability (e.g. see reviews by Hirose (2005) and Hikosaka et al. (2016)). In dense vegetation, plants generally allocate most nutrients and especially nitrogen to leaves that are in the highest, most illuminated parts of the canopy and therefore contribute most to photosynthesis (Hirose et al., 1987). This selective allocation pattern is believed to maximise whole-plant photosynthesis and is believed to be especially important in a competitive environment (Boonman et al., 2006; Izaguirre et al., 2013) where resource gradients are more pronounced, as exemplified by the increased light extinction in dense canopies. Leaves with a high light capture are vital to plant performance in a competitive environment due to their high contribution to plant carbon gain. This high light capture leads to selective investment of limiting resources to maximise the carbon gain from these leaves, further increasing their value. Models based on these principles are now widely used in basic plant ecological research (see Hirose (2005)), as well as in e.g. crop scientific and climate change studies (see Dewar et al. (2009)). However, an issue that these studies often overlook is the feedback that these resource allocation patterns in the canopy have on the distribution of herbivores and defences in the canopy.

The heterogeneity in nutritional value and defensive status of leaves also affects the 
spatial distribution of herbivores in the canopy, potentially threatening plants with the removal of leaves vital to their performance. Herbivore species differ in their susceptibility to taxonspecific secondary metabolites (Bennett \& Wallsgrove, 1994) and can be roughly classified into two types: generalist and specialist species. Generalist species are not particularly resistant to any one secondary metabolite but can feed from a variety of host plants, avoiding those with high levels of secondary chemistry (Feeny, 1976). In contrast, specialist herbivores have adapted their metabolism to be more tolerant to taxon-specific secondary plant metabolites, but require a host of that particular taxon. Similarly, generalists are known to feed on less defended leaves lower in the canopy due to their lower resistance to plant defences, which are concentrated in young leaves in the top of the canopy (Cates, 1980). Conversely, a specialist's increased resistance to plant defences allows them to target the young leaves that in dense vegetation are usually produced in the top of the canopy, which have a higher nutritional value but are also better defended. Therefore, generalist and specialist herbivores potentially have a different impact on the optimal allocation of defences and how growth-defence trade-offs affect plant fitness. However, it is unknown how the spatial distribution of herbivores interacts with competition driven dynamics in resource gain and allocation, and how this impacts plant defence responses and growth-defence trade-offs.

Leaves at the top of the canopy that are preferred by specialist herbivores are vital to plant survival in a competitive environment, potentially aggravating the negative effect of specialist herbivores on plant fitness in such a competitive environment (de Vries et al., 2017). Plants respond to impending competition with a set of morphological changes that maximise light capture in a competitive environment, including enhanced elongation of internodes and petioles, increased leaf angles and reduced branching. This set of responses is termed the shade avoidance syndrome (SAS) (Fraser et al., 2016; Ballaré \& Pierik, 2017), and is mediated by the ratio of red to far-red light in the light spectrum (R:FR), among other signals. This light signal is a solid predictor of impending competition as plant tissues absorb red light, while they reflect and transmit most of the far-red light (Ballaré et al., 1990; Ballaré \& Pierik, 2017). A low R:FR ratio indicates that light conditions are likely to be unfavourable in the near future and is known to negatively affect plant defences (Moreno et al., 2009; de Wit et al., 2013; Izaguirre et al., 2013; Ballaré, 2014; Campos et al., 2016). Competition creates heterogeneity in the light conditions and therefore creates heterogeneity in the distribution of defences in the canopy (Ballaré et al., 2012; Ballaré, 2014). This competition-induced heterogeneity in nutritional value and defensive status of leaves in turn affects the spatial distribution of herbivore damage 
depending on the herbivore species attacking the plant.

The interaction between plant-plant competition and herbivory can thus only be understood in a spatially dynamic context, which is not supported in conventional modelling tools, hampering quantitative analysis. To overcome this, we used the three-dimensional modelling techniques of functional structural plant (FSP) modelling (Vos et al., 2010) as a tool to assess how competition affects plant susceptibility to herbivores with different levels of specialization. FSP modelling is a promising tool to study plant-plant-herbivore interactions (de Vries et al., 2017). The FSP model used in this study is novel through the incorporation of several interacting dynamic processes which are applied to the novel field of modelling plantherbivore interactions. The model dynamically simulates plant-plant-herbivore interactions by implementing mechanistic source-sink-driven plant growth (Evers, 2016), R:FR-mediated architectural development (Bongers et al., 2014) and the introduction of herbivores using agentbased modelling concepts. This allowed us to model both the direct effects of competition and herbivory on plant performance, as well as the indirect effects resulting from the interaction between herbivory and competition. First, we define our model and then address the question how generalist and specialist herbivores affect plant fitness at varying levels of competition.

\section{Materials and Methods}

In this study we conducted two field experiments that serve to parameterise and validate the functional-structural plant (FSP) model on plant growth and herbivory, and one greenhouse experiment to parameterise caterpillar feeding and growth. Model performance was evaluated by comparing simulations with experimental data, after which the model was used to elucidate the interaction effect of herbivory and plant competition for light.

\section{Experimental design}

To parameterise and validate the model we conducted 2 field experiments in 2014 and 2015 in Wageningen, the Netherlands. Plants of B. nigra were planted in three densities $(1,4$ and 25 plants $\mathrm{m}^{-2}$ ) in plots of $2 \times 2$ meters, with 3 replicates per density. Seeds were germinated in a greenhouse compartment with a 16/8 light/dark photoperiod $\left(20-22{ }^{\circ} \mathrm{C}, 50-70 \%\right.$ relative humidity) and transferred to soil cubes as seedlings before being planted at 2 weeks old on the $21^{\text {st }}$ of May (both years). Plots were weeded and sprayed with herbicides and pesticides weekly to exclude interspecific competition and herbivores. In 2014 , three plants per plot ( $\mathrm{n}=9$ plots per treatment) were randomly selected for weekly non-destructive measurements of plant height, total number of leaves along the main stem, the length and width of those leaves and the 


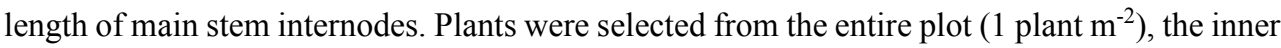
$3 \times 3$ plants $\left(4\right.$ plants $\left.\mathrm{m}^{-2}\right)$ or the inner $4 \times 4$ plants $\left(25\right.$ plants $\left.\mathrm{m}^{-2}\right)$ to minimize border effects on measured values. Single measurements on leaf angles, branching angles, ear development, photosynthetic capacity and light quality (R:FR) were conducted on different moments during the experiment (see Methods S1). At the end of the experiment we counted the number of branches per plant, measured internode thickness along the main stem and at branch bases and harvested seeds to determine yield and average seed weight. Gathering seeds for yield measurements prevented us from sampling plants for destructive measurements during the first experiment, which is why we repeated the experiment in 2015. In this year we quantified plant biomass at 124 days after sowing, which was after the onset of flowering but before all leaves senesced (Figure S3.1). We also measured internode tissue density, which was done by measuring the internodes length and radius to determine the internode volume and weighing the internodes after a 16 hour drying period in a $70{ }^{\circ} \mathrm{C}$ oven.

Additionally we conducted a greenhouse experiment to parameterise caterpillar feeding and growth based on the small cabbage white (Pieris rapae; Pieridae). This is a specialist on Brassicaceae and a caterpillar average in terms of size and its feeding damage compared to other insect herbivores on $B$. nigra. The caterpillars originated from the stock rearing of the Laboratory of Entomology, Wageningen University, where they are maintained on Brussel sprouts plants (B. oleracea var. gemmifera L. cultivar Cyrus) in climatised rooms at $20-22{ }^{\circ} \mathrm{C}$, $50-70 \%$ relative humidity and a 16/8 light/dark photoperiod. We measured plant damage and subsequent growth of 30 individual caterpillars in their development from first instar to pupation while feeding on leaves of B. nigra, which were grown in the same conditions as the Brussel sprouts the caterpillars were reared on. The caterpillars were kept in Petri dishes in a greenhouse compartment with a $16 / 8$ light/dark photoperiod and a $20: 16^{\circ} \mathrm{C}$ temperature cycle. We placed a young leaf cut from a B. nigra plant grown in the same greenhouse in the Petri dishes, with a piece of wet cloth wrapped around the stem to maintain leaf quality. The leaves were photographed before being placed in the Petri dishes and after two days of feeding so that the initial and damaged leaf area could be determined using ImageJ (Figure S3.2). Until pupation, every two days the caterpillars were weighed and the leaves refreshed. Only the caterpillars that turned into healthy pupa (determined by colour and weight) were used for data analysis. The measurements were used to determine herbivore feeding rate in $\mathrm{m}^{2}$ leaf damage per g caterpillar mass per growing degree day (to correct for the effect of temperature on feeding rate), the caterpillar growth in $\mathrm{g}$ per $\mathrm{m}^{2}$ of consumed leaf area, the caterpillar weight at which 
pupation was initiated and the time to pupation (Table 2.).

\section{Model design}

The model used in this study was designed to mechanistically simulate plant-plant-herbivore interactions using a three-dimensional FSP modelling approach (Vos et al., 2010). The model was implemented in the platform GroImp v1.5 (Hemmerling et al., 2008) and simulated individual plants growing at various densities in which plants compete with their neighbours for light. The distribution of light interception in the 3D scene was simulated using a MonteCarlo pathtracer embedded in GroImp (Hemmerling et al., 2008). The light environment was modelled using both randomly arranged diffuse light sources and direct light sources spread over the solar path that takes latitude and the simulated day of year into account (Evers 2010). Border effects in the light environment were eliminated by replicating a plot of simulated plants 25 times in the $\mathrm{x}$ and $\mathrm{y}$ directions for the light model calculations. The light conditions experienced by the 625 copies of each individual plant were then averaged, effectively eliminating border effects. Limitation of growth by belowground resources such as water and nutrients was not considered. The model consisted of several components: plant architectural development, carbon assimilation, allocation and growth, shade avoidance, and herbivore behaviour and growth. An elaborate technical description of the implementation of these mechanisms can be found in the model design supplement (see Methods S2).

\section{Plant architectural development}

Following FSP modelling principles (Evers, 2016), the plant architecture was represented using a repetition of elementary units called phytomers, which consist of an internode, a leaf and an axillary meristem. Vegetative phytomers were sequentially produced at the top of the growth axis by the shoot apical meristem. After having produced a set number of vegetative phytomers, the plant went into flowering and the meristem produced an inflorescence, after which no further vegetative phytomers were produced on the shoot. Axillary meristems could grow and develop similar to the apical shoot meristem to form branches. Branch initiation and abortion was simulated using cues that are related to apical dominance, assimilate availability and light quality (Evers et al., 2011). The outgrowth of axillary meristems is delayed by the dominance of the apical meristem as long as it remained vegetative or until the axillary meristem reached a given age. Additionally, a meristem required favourable growing conditions (surplus assimilates) to break and develop into a branch, which could be aborted during its development in case of unfavourable conditions (low assimilates, low R:FR). Details on the mechanisms of 
branch initiation and abortion and their implementation can be found in supplement S4. Branching also determined internode thickness as this was modelled using a pipe model, where the cross-sectional surface area of an internode is equal to the sum of the cross-sectional area of daughter internodes. This was relevant for model performance as internode thickness drove allocation of assimilates to the stem, which was a major sink that competed for assimilates with leaves and seeds.

\section{Carbon assimilation, allocation and growth}

The leaf area of each plant in the simulated plot determined light interception and subsequent carbon assimilation through photosynthesis, which then fed back to plant growth and leaf area expansion. Therefore, the effect of competition for light on plant growth was an essential, yet emergent, property of this FSP model, and depended on plant density and canopy structure. The amount of photosynthetically active radiation (PAR) absorbed by each individual leaf was calculated using reflectance and transmittance coefficients generally applicable to plant tissue (Jacquemoud \& Baret, 1990). Leaf carbon assimilation was calculated from the amount of PAR absorbed by the leaf and its basic photosynthetic capacity, which itself was a function of the fraction PAR absorbed (Niinemets \& Anten, 2009; Hikosaka et al., 2016). Assimilates were then stored in a central carbon pool from which maintenance respiration and assimilates needed for growth were deducted, and any remainder was kept as stored resources. Assimilates were distributed within the plant based on the relative sink strengths of organs, e.g. the assimilates needed to achieve potential growth (sink) relative to the sum of sink strengths of all growing organs in the plant (Heuvelink, 1996). This assumed no hierarchy for assimilate allocation between organs and took the added costs of growth respiration into account. The potential growth of an organ was described using the beta function for sigmoid growth (Yin et al., 2003).

\section{Shade avoidance}

We used the light model to simulate the ratio between absorbed red and far-red light (R:FR) using reflectance and transmittance coefficients for red and far-red light generally applicable to plant tissue (Jacquemoud \& Baret, 1990). This R:FR signal was perceived by the plant on the leaf tip and used to mediate both local and systemic integration of the shade avoidance response. Local mediation happens at the phytomer level, where the R:FR perceived by a leaf tip (Pantazopoulou et al., 2017) mediated the shade avoidance response in that leaf as well as in the adjacent internode and lateral meristem. The R:FR signal mediated leaf width, leaf angle and internode length using a dose-response curve, while it mediated meristem outgrowth and 
abortion using a threshold method. A systemic integration of the combined R:FR signal of all main stem leaves regulated flowering time and potential leaf length, both using a threshold method of signal integration (Evers et al., 2007).

\section{Model calibration}

We measured a number of parameters regarding plant architecture, development and growth that describe the plant phenotype in two different plant densities and serve as input to the FSP model (Table 1). These parameter values serve as a baseline description of $B$. nigra growth and development, based on the low density phenotype. However, a number of growth parameters could not be measured directly in the field. To obtain values for these parameters we used an optimisation procedure where model output was fitted to measured data. Potential growth

Table 3.1. Model parameters describing plant architectural development obtained through measurements or optimisation.

\begin{tabular}{|c|c|c|c|}
\hline Parameter & Value & Unit & Acquisition \\
\hline Leaf growth period & 234 & gdd & Measured \\
\hline Leaf growth rate peak & 167 & gdd & Measured \\
\hline Leaf length/width ratio & $1.756(2.5)^{*}$ & dimensionless & Measured \\
\hline Leaf mass per unit area & 65.8 & $\mathrm{~g} \mathrm{~m}^{-2}$ & Measured \\
\hline Internode growth period & 258 & gdd & Measured \\
\hline Internode growth peak & 216 & gdd & Measured \\
\hline Initial internode radius & 0.0008 & $\mathrm{~m}$ & Measured \\
\hline Internode density & 176142 & $\mathrm{~g} \mathrm{~m}^{-3}$ & Measured \\
\hline Leaf appearance rate & 12 & gdd & Measured \\
\hline Number of phytomers on the main stem & $29 \pm 5^{* *}(27 \pm 1)^{*}$ & dimensionless & Measured \\
\hline Branch angle & 40 & degrees & Measured \\
\hline Leaf angle & $70(15)^{*}$ & degrees & Measured \\
\hline Base photosynthetic capacity & 30 & umol m $\mathrm{m}^{-2} \mathrm{~s}^{-1}$ & Measured \\
\hline Potential leaf length & $0.285 \pm 0.026^{* *}$ & $\mathrm{~m}$ & Optimised \\
\hline Leaf length reduction by RFR & 0.55 & dimensionless & Optimised \\
\hline Potential internode length & $0.045 \pm 0.01^{* * *}$ & $\mathrm{~m}$ & Optimised \\
\hline Internode length increase by RFR & 3.5 & dimensionless & Optimised \\
\hline Midpoint of the R:FR response & 0.85 & dimensionless & Optimised \\
\hline Steepness of the R:FR response & 30 & dimensionless & Optimised \\
\hline RFR threshold for branch abortion & 0.65 & dimensionless & Optimised \\
\hline
\end{tabular}

* A value in brackets denotes a separate parameter value is used for the high density phenotype.

** Parameter drawn from a normal distribution with a mean and a standard deviation, indicated with the mean \pm sd notation. 
Table 3.2. Model parameters describing herbivore feeding, growth and development based on Pieris rapae, a medium sized caterpillar commonly found on Brassica nigra plants. Caterpillar weight gain $\left(c_{w}\right)$ is modelled using a two parameter exponential function of caterpillar feeding rate $\left(c_{f}\right)\left(c_{w}=a_{w} c_{f}^{b}\right)$, and caterpillar feeding rate is modelled using a linear function of caterpillar leaf area intake $\left(c_{l}\right)\left(c_{f}=a_{f} c_{l}\right)$.

\begin{tabular}{lll}
\hline Parameter & Value & Unit \\
\hline$a_{w}$ & 10.61 & $\mathrm{~g} \mathrm{~m}^{-2}$ \\
$b$ & 0.7747 & unitless $^{-1}$ \\
$a_{f}$ & $7.68 \cdot 10^{-4}$ & $\mathrm{~m}^{2} \mathrm{~g}^{-1} \mathrm{gdd}^{-1}$ \\
Initial herbivore weight & $1.86 \cdot 10^{-5}$ & $\mathrm{~g}$ \\
Maximum herbivore weight & 0.178 & $\mathrm{~g}$ \\
Herbivore life span & 210 & $\mathrm{gdd}$ \\
\hline
\end{tabular}

parameters of leaves and internodes as well as the effect of R:FR on these parameters were optimised such that the simulated biomass, yield, plant height and leaf length distribution patterns mimicked observed values. Note that organ size and, therefore, plant size and leaf area were thus model output rather than input, as they were the combined result of several growth parameters, mediation by R:FR, as well as carbon availability throughout plant development.

\section{Herbivore behaviour and growth}

The simulated caterpillars were simulated as individual agents in the $3 \mathrm{D}$ scene, and used random probability selection to choose a host-plant leaf upon instantiation and movement events. Every leaf was given a value that represented the relative probability $(r P)$ that the leaf was selected by the individual herbivore. This value was described using a sigmoid curve based on temperature corrected leaf age (leafAge), in growing degree days ( $g d d$ ), the herbivore feeding preference $(h, 0-1)$ and the parameters $r P_{\max }$ (max relative probability, denotes the difference between the youngest and oldest leaves), $s$ (steepness parameter, determines the slope of the sigmoid curve for a given value of $h$ ) and $m$ (midpoint of the sigmoid curve) (Figure S3.3).

$$
r P=\frac{r P_{\max }}{1+\exp (s *(h-(1-h)) *(\text { leaf Age }-m))}
$$

Once the herbivore had chosen a host leaf, herbivore feeding rate was described using a linear relation with herbivore biomass (Table 2. Herbivore feeding rate). The herbivore then grew following a linear function based on the consumed leaf area (Table 2. Herbivore growth rate a \& b). When a leaf senesced, the herbivore moved to a new host leaf, had less biomass than the herbivore could consume during a time step, or in case the herbivore developed into the 
next instar, which happened four times during its development at regular intervals. The herbivore pupated when it reached pupal age (Table 2. Herbivore life span), or when the herbivores mass reached a pupation threshold (Table 2. Maximum herbivore weight), effectively removing the herbivore from the 3D scene. The simulated herbivore types differed in their feeding preference for older $(h<0.5)$ or younger leaves $(h>0.5)$, as is typical for the feeding behaviour of generalist and specialist herbivores respectively.

\section{Model evaluation}

To evaluate model performance, we compared model output with measured data from the field experiments on the optimised variables leaf length, internode length and number of branches, as well as yield and biomass. The latter were validation variables of a higher integration level that resulted from the functional mechanisms of plant morphology, carbon allocation and shade avoidance. For example, plant biomass was largely determined by leaf area and branching patterns (Figure S3.4) which were variables at a lower level of integration. Complementary to the low and high density we also used an intermediate plant density ( 4 plants $\left.\mathrm{m}^{-2}\right)$ that was not used in model calibration to evaluate model performance. The simulations for model evaluation mimicked the setup of the field experiments; we simulated three plant densities: low (1 plant $\left.\mathrm{m}^{-2}\right)$, intermediate $\left(4\right.$ plants $\left.\mathrm{m}^{-2}\right)$ and high $\left(25\right.$ plants $\left.\mathrm{m}^{-2}\right)$. The simulations were conducted with 4, 4 and 16 plants per plot for the respective plant densities. These were replicated 625 times for the calculation of light interception distribution, resulting in plots of 2500, 2500 and 1000 simulated plants. Simulations ran from the $31^{\text {st }}$ of March to the $2^{\text {nd }}$ of August (124 days), with average daily temperature, average daily insolation and solar angle typical for the Netherlands at a latitude of 52 degrees. The output gathered from these simulations were LAI and R:FR (daily), leaf lengths, plant height, biomass and yield (after 124 time steps). Output from all plants in the plot was averaged and 10 replicates were simulated. 


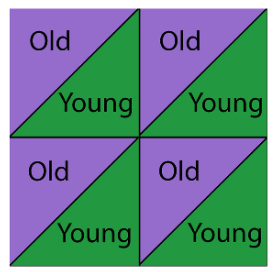

a

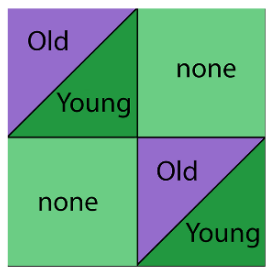

b

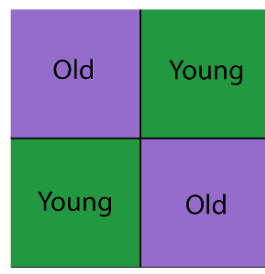

C

Figure 3.1. Three herbivore distribution patterns visualised with a block of 4 plants: a) A homogeneous herbivore distribution where all plants are infested with the same herbivore type (i.e., herbivores preferring either old or young leaves). b) A heterogeneous herbivore distribution following a checkerboard design of plants infested with the same herbivore type and non-infested plants. c) An alternating herbivore distribution following a checkerboard design of plants infested with herbivores preferring young leaves and plants infested with herbivores preferring old leaves.

\section{Simulations}

To investigate how herbivore feeding behaviour influences plant yield and interacts with plant competition for light we conducted a full factorial set of simulations. These consisted of two plant densities (low, 1 plant $\mathrm{m}^{-2}$; high, 25 plants $\mathrm{m}^{-2}$ ), two herbivore types with different feeding preferences (preference for old leaves, $h=0.2$; preference for young leaves, $h=0.8$,), and three herbivore distributions: i) A homogeneous herbivore distribution where all plants were infested with the same herbivore type to get a baseline effect of herbivory on plant phenotype at a given plant density (Figure 3.1a). ii) A heterogeneous herbivore distribution following a checkerboard design of plants infested with the same herbivore type and non-infested plants to test the interactive effect of herbivore damage and plant competition (Figure 3.1b). iii) An alternating herbivore distribution following a checkerboard design of plants infested with herbivores preferring young leaves and plants infested with herbivores preferring old leaves to test the effect of these two herbivore types on plant competitiveness (Figure 3.1c). Plots contained four plants in the low density and 16 plants in the high density, and simulations ran from the $31^{\text {st }}$ of March to the $2^{\text {nd }}$ of August (124 days), with average daily temperature, average daily insolation and solar angle typical for the Netherlands at a latitude of 52 degrees. 5, 10, 15 or 20 herbivores per plant were introduced after 60 days of growth $(230 \mathrm{gdd})$, at this time the plants had accumulated enough leaf area to sustain the herbivores and the plant phenotypes in low and high plant densities were still equal (Figure S3.5). The range in the number of simulated herbivores was chosen to represent the range of herbivores occurring in natural settings (Poelman et al., 2009). We kept the number of herbivores per plant constant 

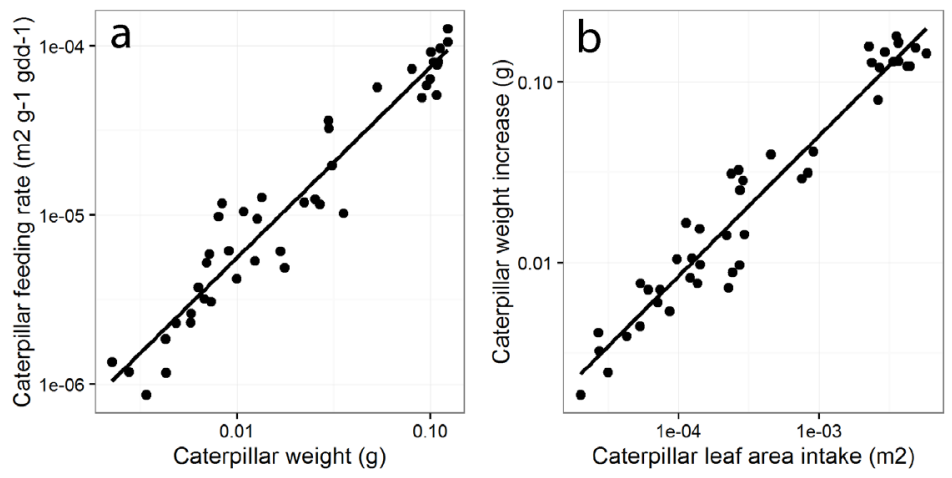

Figure 3.2. Correlation between measured data on caterpillar weight $(\mathrm{g})$ and caterpillar feeding rate $\left(\mathrm{m}^{2}\right.$ leaf area $\mathrm{g}$ caterpillar weight $\left.{ }^{-1} \mathrm{gdd}^{-1}, \mathrm{R}^{2}=0.92\right)(\mathrm{a})$ and between caterpillar leaf area intake $\left(\mathrm{m}^{2}\right)$ and subsequent caterpillar weight gain $\left(\mathrm{g}, \mathrm{R}^{2}=0.94\right)(\mathrm{b})$

and equal for the two densities. Alternatives such as keeping the number of herbivores constant per unit ground area would result in either unrealistically high herbivore numbers on low density plants (500 herbivores per plant) or irrelevantly low herbivore numbers on high density plants ( 0.2 herbivores per plant). The output gathered from these simulations was cumulative herbivore damage, number of branches, leaf area, plant height, biomass and yield (after 124 days). The output of all plants in the plot was averaged and each treatment replicated 5 times. The model output was tested for significance by conducting an analysis of variance (ANOVA) on the coefficients of a linear regression model. We tested for significance at the $5 \%$ probability level. All average values are accompanied by a standard error, either through error bars in graphs or in textual form (mean \pm se).

\section{Results}

\section{Model calibration}

A key feature of the model is simulation of the dynamic adjustment of the architectural phenotype in response to changes in plant density through changes in the light environment. Six variables that have a large impact on plant phenotype were observed to change with plant density in our experiments: leaf length distribution along the main stem, leaf angle, internode length, the number of phytomers in the main stem and the number of branches on the main stem. For our model, we assumed that these variables were all mediated by changes in R:FR as these responses fall within the framework of the shade avoidance syndrome (Ballaré \& Pierik, 2017). The model was capable of simulating distinct differences in the R:FR ratio over time 

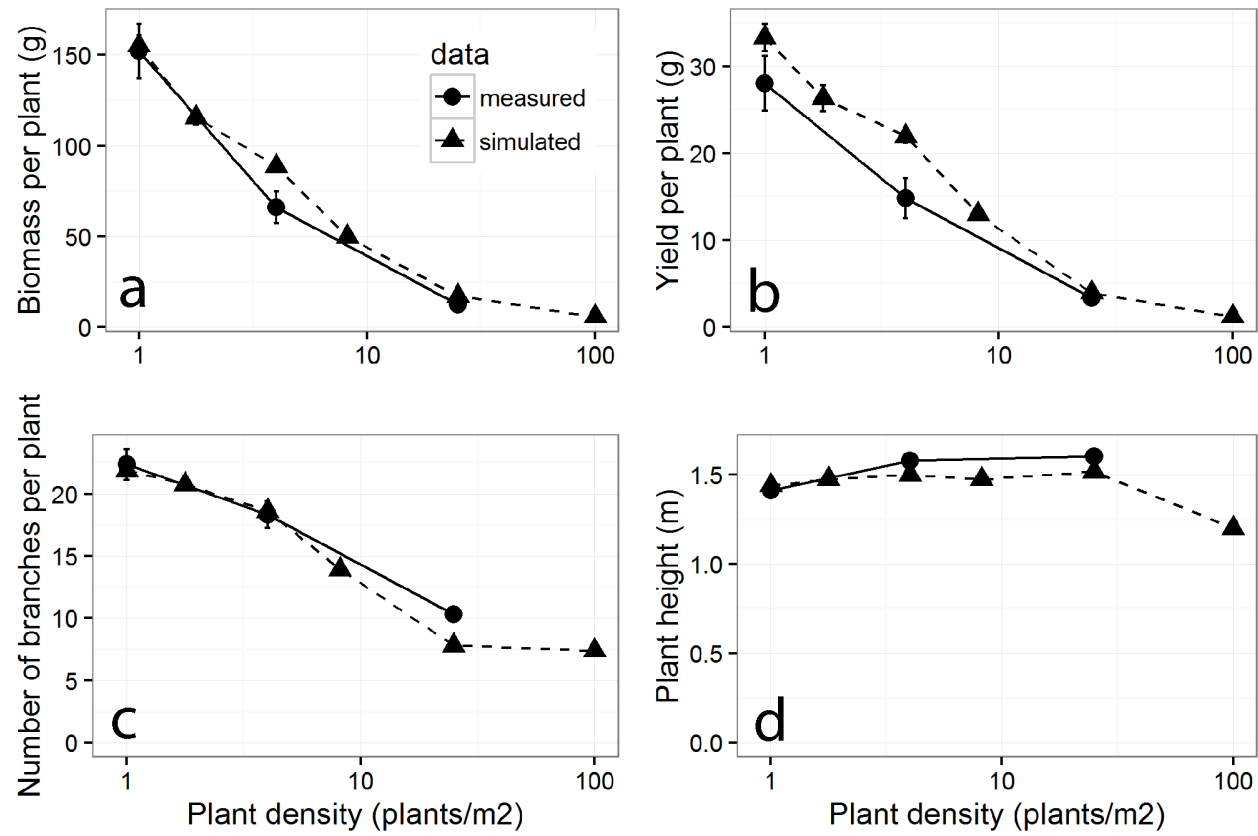

Figure 3.3. Comparison of measured (circles and solid lines) and simulated data (squares and dashed lines) on plant biomass (a), plant yield (b), number of branches (c) and plant height (d) as a function of plant density. The measured data is collected at 3 plant densities whereas the simulated data covers 6 plant densities, showing the strength of model predictions beyond the parameterised densities ( 1 and 25 plants $\mathrm{m}^{-2}$ ).

between different plant densities (Figure S3.6). We optimized the simulated plant responses to a change in R:FR (Table 1) so that the phenotype of a simulated plant in high density represented the high-density phenotype observed in the field experiment.

\section{Herbivore feeding}

Measured caterpillar weight $(\mathrm{g})$ was strongly correlated with caterpillar feeding rate $\left(\mathrm{m}^{2} \mathrm{~g}^{-1}\right.$ $\operatorname{gdd}^{-1}$, Figure 3.2a). Subsequently, the amount of leaf area consumed by the caterpillar $\left(\mathrm{m}^{2}\right)$ was strongly correlated with caterpillar weight gain (g, Figure 3.2b). We parameterised the simulated caterpillars by fitting a function to the measured data; we used a linear function $(\mathrm{y}=$ $a x$, Table 2.) to simulate caterpillar feeding rate as a function of caterpillar weight and a two parameter power function $\left(\mathrm{y}=a x^{b}\right.$, Table 2.) to simulate caterpillar weight gain as a function of caterpillar leaf area intake. 

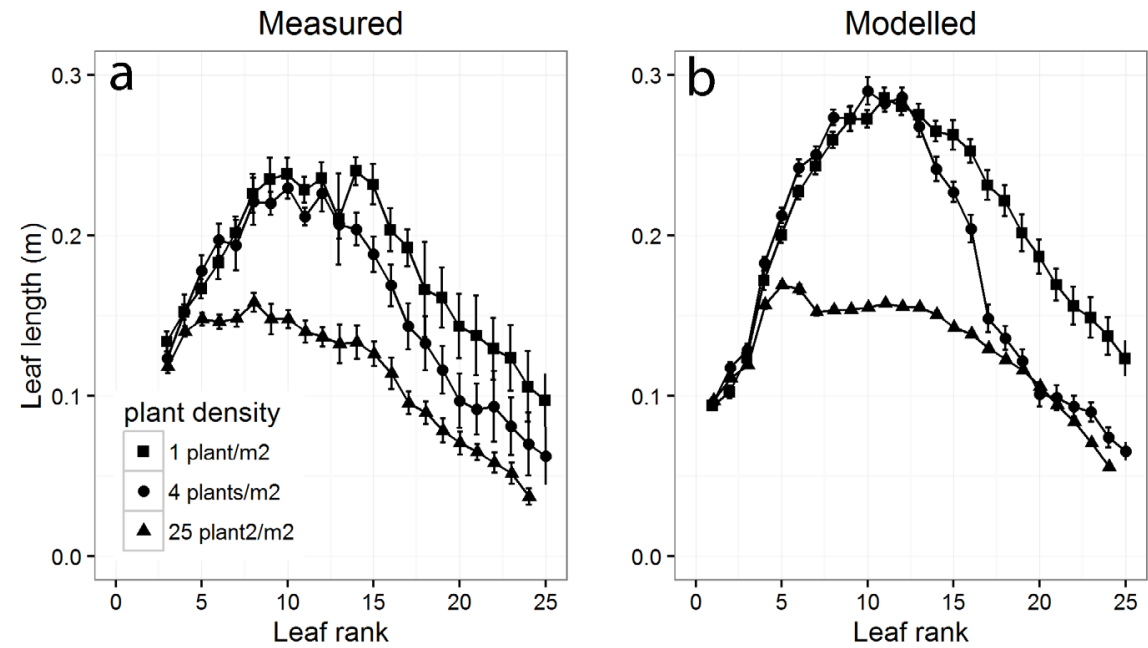

Figure 3.4. Leaf length per leaf rank on the main stem of Brassica nigra grown in three densities in the field (a) and simulated with the model (b). The high ( 25 plants $\left.\mathrm{m}^{-2}\right)$ and low densities $\left(1\right.$ plant $\left.\mathrm{m}^{-2}\right)$ have been used for model calibration while the middle density ( 4 plants $\mathrm{m}^{-2}$ ) served as validation. Up to leaf rank 25 is shown as not all plants produce enough phytomers to have leaves of higher ranks along the main stem.

\section{Evaluation of model performance}

Plant biomass and yield of seeds were used as proxies for plant performance and fitness and were therefore our targets for evaluation of model performance. Measured plant biomass ranged from an average $152.2 \pm 4.3 \mathrm{~g}$ dry weight at low density to $12.4 \pm 0.2 \mathrm{~g}$ at high density (Figure $3.3 \mathrm{a})$ and measured plant yield showed a similar pattern ranging from $28 \pm 3.2 \mathrm{~g}$ dry seed weight at the low density to $3.3 \pm 0.52 \mathrm{~g}$ at the high density (Figure $3.3 \mathrm{~b}$ ). Simulated plants showed comparable correlation between plant density and both plant biomass and yield, with a slight overestimation of biomass and yield at an intermediate density.

The number of branches on the main stem was one of the main determinants of simulated plant biomass $\left(\mathrm{R}^{2}=0.72\right.$, Figure S3.4) and yield of seeds $\left(\mathrm{R}^{2}=0.70\right.$, Figure S3.4). The measured number of branches decreased with plant density from $22.4 \pm 1.2$ at low density to $10.6 \pm 0.5$ at high density (Figure $3.3 \mathrm{c}$ ). Our FSP model was able to mimic the observed nonlinear decrease of the number of branches with plant density by mediating the abortion of developing branches by the drop in simulated R:FR associated with increasing plant density (Figure S3.6). 

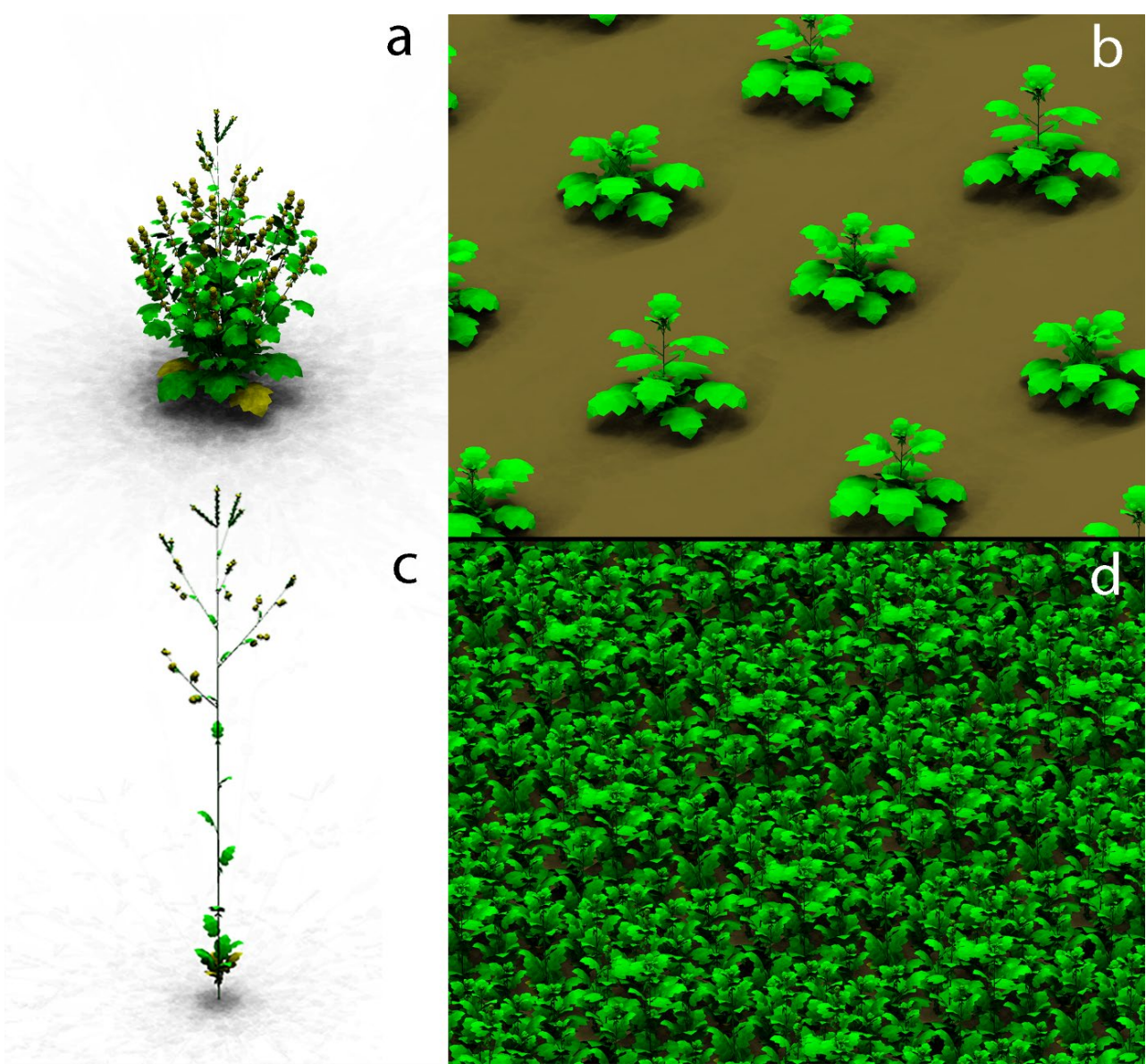

Figure 3.5. Simulated plant phenotype in a low density of 1 plant $/ \mathrm{m} 2(\mathrm{a}, \mathrm{b})$ and a high density of 25 plants $/ \mathrm{m} 2(\mathrm{c}, \mathrm{d})$, showing isolated plants in the generative stage $(\mathrm{a}, \mathrm{c})$ and a field of simulated plants in the vegetative stage $(b, d)$.

Measured plant height ranged from $1.41 \pm 0.04 \mathrm{~m}$ in the low plant density to $1.6 \pm 0.04 \mathrm{~m}$ in the high density. The simulated plants had a height of $1.48 \mathrm{~m}$ at low density to $1.28 \mathrm{~m}$ at high density (Figure 3.3d). Model underestimation of plant height at higher densities was potentially due to a border effect that was present in the measured plants but was absent in the model simulations. However, plant height contributed relatively little to the accumulation of biomass $\left(\mathrm{R}^{2}=0.066\right.$, Figure S3.4) and yield $\left(\mathrm{R}^{2}=0.12\right.$, Figure S3.4) and was therefore given less weight when optimising model performance.

Leaf area was another main determinant of simulated plant biomass $\left(\mathrm{R}^{2}=0.94\right.$, Figure S3.4) and seed yield $\left(\mathrm{R}^{2}=0.92\right.$, Figure S3.4) in the model. In field grown plants we observed 
differences in leaf length distributions along the main stem between densities; plants grown at high density had shorter leaves and a different length distribution along the main stem than plants grown in low density (Figure 3.4). The leaf rank at which the leaf length values of plants from different densities deviated from each other give a relative indication of the stage in plant development at which canopy closure initiated shade avoidance (Figure S3.6, S3.7). Although leaf lengths were slightly overestimated by the model, the simulated pattern in the effect of plant density on leaf length distributions closely matched field data. Accurate depiction of this density effect on leaf length distributions is instrumental to evaluate the interaction between herbivory and plant density.

In conclusion, the effect of plant density on the simulated plants was comparable to the effect of plant density on real plants. Both the simulated yields, which we used as a proxy for plant fitness, as well as the simulated variables that underlie the accumulation of yield (leaf length, branching patterns, biomass, Figure S3.4) showed patterns comparable to real plants. Therefore we conclude that the model satisfactorily simulated the most important mechanisms that determined changes in plant phenotype in response to changes in plant density (Figure 3.5).

\section{The effect of herbivory on plant performance}

Using the model, we tested how herbivore feeding behaviour influenced plant yield and interacts with plant competition for light by simulating different levels of herbivory in two plant densities, two herbivore feeding preferences and three herbivore distribution patterns.

From our simulations a clear interaction effect between herbivory and plant density emerged (Figure 3.6). Herbivory had no significant effect on simulated plant yield in any of the low density treatments (Figure 3.6a,c,e), whereas herbivory had a significant negative effect on simulated plant yield in all high density treatments (Figure 3.6b,d,f). This interaction between herbivory and density can be explained by three factors. First, low density plants had considerably more leaf area (Figure S3.5), branches and biomass (Figure 3.3a,c) than the high density plants. These size differences decreased the relative impact of a single herbivore on low density plants, which allowed the low-density plants to compensate damage more readily than 


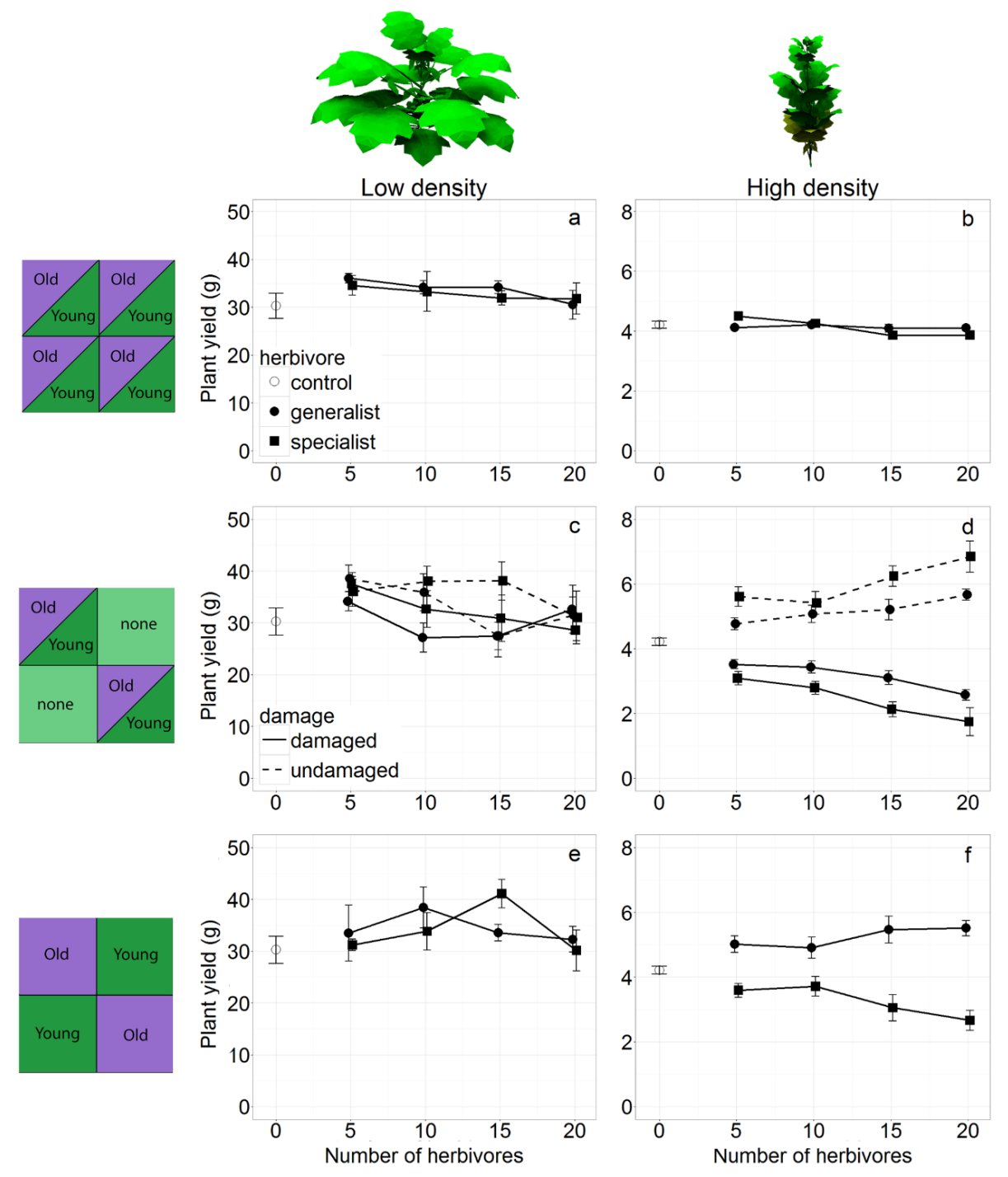

Figure 3.6. Simulated plant yield at day 124 plotted against the number of herbivores per plant in two densities (low: 1 plant $\mathrm{m}^{-2}$, high: 25 plants $\mathrm{m}^{-2}$ ) and three herbivore distribution patterns: Homogeneous distribution of herbivores preferring either old or young leaves $(a \& b)$, a checkered pattern of undamaged plants (none) and plants infested by herbivores preferring old or young leaves (c \& d) and a checkered pattern of plants infested by herbivores preferring old or young leaves (e \& f). $\mathrm{N}=5$ in all cases.

the high density plants. Second, the number of herbivores per plant was kept constant, resulting in more herbivores per unit ground area and plant biomass at the high density. Third, the increased competition among plants in high densities magnified small herbivore-induced 
differences in plant biomass between neighbouring plants because of the resulting asymmetry in competitive strength. Additionally, what happened to the light interception lost by leaves because of herbivore damage should be considered. In low densities some of that light was not intercepted by the plants at all, but most was intercepted by other leaves of the same plant instead. In the high plant density however, part of that light was intercepted by other leaves of the same plant, whereas another part of that light was intercepted by leaves of other plants. This spillage of light to neighbouring plants gave them a competitive advantage over the damaged plant and caused asymmetry in the competition between these plants.

The number of herbivores and the herbivore feeding preference had no effect on plant yield in a low plant density, regardless of the distribution of the herbivores. In a homogeneous distribution of herbivores in a high plant density (Figure 3.6b), the number of herbivores had a small negative effect on plant yield $(\mathrm{F}=14.97, \mathrm{P}<0.01)$ and herbivore feeding preference had a small effect $(\mathrm{F}=6.41, \mathrm{P}<0.05)$. Additionally, herbivore density and feeding preference interacted $(\mathrm{F}=7.02, \mathrm{P}<0.01)$, where herbivores preferring old leaves suppressed yield more than herbivores preferring young leaves at low herbivore abundance and vice versa at high herbivore abundance. Although all these effects are highly significant, the relevance of these effects can be disputed as the magnitude of these effects was not very large. When infested plants were competing with uninfested neighbours (Figure 3.6d) the number of herbivores suppressed the yield of infested plants $(\mathrm{F}=28.11, \mathrm{P}<0.001)$. The herbivore feeding preference also affected the yield of infested plants $(\mathrm{F}=19.05, \mathrm{P}<0.001)$, where herbivores preferring young leaves supressed yield more than herbivores preferring old leaves. However, the number of herbivores and herbivore feeding preference did not interact. Having both infested and uninfested plants within a plot created a competitive disadvantage for infested plants and a competitive advantage for uninfested plants, because the uninfested plants had more leaf area than the infested plants.

This variation in leaf area and, consequently, in competitive strength was magnified by competition and translated in a yield difference that increased with the number of herbivores per plant. Additionally, this effect was greater for plants infested with herbivores preferring young leaves at the top of the canopy compared to herbivores preferring old leaves in the bottom of the canopy ( $h, \mathrm{Eq} 1)$. With a checkered distribution of plants infested with herbivores preferring young leaves and herbivores preferring old leaves (Figure 3.6f) the herbivore feeding preference affected plant yield $(\mathrm{F}=80.48, \mathrm{P}<0.001)$ and interacted with the number of herbivores $(\mathrm{F}=7.79, \mathrm{P}<0.01)$. The herbivores preferring old leaves had a positive effect on plant 
yield that increased with the number of herbivores whereas the herbivores preferring young leaves had a negative effect on plant yield that increased with the number of herbivores. The herbivores feeding on young leaves in the top of the canopy caused their host plants to have a competitive disadvantage compared to the plants infested with herbivores that fed on older leaves in the bottom of the canopy $(h, \mathrm{Eq} 1)$.

\section{Discussion}

Our results indicate that the costs of herbivory for plants may strongly depend on the type of herbivore attacking the plant and the extent of plant-plant competition under which plants are attacked by herbivores. Our model predicts that damage either by herbivores feeding on younger leaves or herbivores feeding on older leaves can be tolerated by plants growing in a low density. In a high plant density the costs of herbivore attack are shaped by the interaction of herbivore feeding location and distribution of herbivory among neighbouring plants. Damage to both young and old leaves can be tolerated when neighbouring plants are equally damaged in both feeding location and density of the herbivores. However, damage to young leaves is more costly to the plant than damage to old leaves when neighbouring plants are less affected by herbivore damage, which causes a competitive advantage and a subsequent fitness benefit for the neighbouring plants. These conclusions show the need to link research on herbivory to research on plant-plant interactions, as the fitness costs and benefits of herbivory and defence are tightly linked to the plant's competitive context.

Many experimental studies have reported that tissue loss from aboveground herbivores affects plant-plant competition by decreasing the competitive ability of one of the competitors (Rees \& Brown, 1992; Hambäck \& Beckerman, 2003; Haag et al., 2004; Schadler et al., 2007). Other studies have shown that preferential feeding of the herbivore impacted plant-plant interactions by increasing the competitive asymmetry between competing plants (Bentley \& Whittaker, 1979; Kim et al., 2013; Borgström et al., 2016). These findings are in concordance with our results that show how a heterogeneous distribution of herbivores between plants impacts plant fitness by affecting the outcome of plant-plant competition. However, aboveground feeding by a generalist herbivore has been shown to affect plant performance without affecting the outcome of plant-plant competition (Jing et al., 2015). These finding are in line with our model predictions as herbivores with a preference for older leaves can be seen as analogous to the feeding behaviour of a generalist herbivore. Future experimental work will have to further validate our model predictions and explore their implications in a complex 
ecological setting.

Young leaves at the top of the canopy represent current resource investment and future resource capture and are, therefore, of high value to the plant. An increase in plant density reduces light capture of older leaves located in lower strata of the canopy and thereby increases the plant's dependency on young leaves for future resource capture. Removal of these disproportionally valuable leaves by a herbivore robs the plant of multiple important resources (e.g. light, nitrogen), which potentially creates a strong competitive disadvantage relative to the neighbouring plants if those are not or less severely attacked. Even a small competitive disadvantage can lead to a fitness loss for the damaged plant through the asymmetry of plant competition. Our results show both the disproportionally high value of young leaves under severe competition and the enhanced negative effect through the asymmetry of light competition when losing these leaves (Figure 3.6). However, current models that relate resource allocation among leave to canopy photosynthesis, growth and competition (e.g. see review Hirose (2005)) generally do not consider the risks and impact of herbivory. Additionally, we can consider how digestion of older versus younger leaves has potential consequences for nutrient competition among plants through changes in litter composition. Secondary metabolites have been shown to serve alternative ecosystem functions, such as the role of tannins in nutrient cycling and retention through their effect on litter decomposition rates (Hättenschwiler \& Vitousek, 2000). Therefore we advocate a stronger quantitative integration of herbivory and defence into existing resource allocation models, models of plant-plant competition and nutrient flux models.

FSP models have been suggested as a useful tool to model dynamic interactions between plants (Vos et al., 2010; Bongers et al., 2014; de Vries et al., 2017). However, most studies to date utilise a limited range of the possibilities provided by FSP modelling: Some studies used descriptive FSP models to test static architectural traits (Chen et al., 2014; Zhu et al., 2015) or a dynamic response to an environmental variable of one architectural trait such as tillering to R:FR (Evers et al., 2007). Other studies included dynamic growth rules based on carbon acquisition and allocation following source-sink principles, but do not include responses to environmental or neighbour-induced signals (Evers et al., 2010; Lopez et al., 2010; Evers \& Bastiaans, 2016) or simulate plants in a static context (Kang et al., 2012). For the present study we developed a fully dynamic FSP model that simulated multiple plants competing for light, both through carbon acquisition and source-sink principles as well as responses to a 
dynamically changing R:FR signal. This way we captured the key mechanisms underlying growth and development of Brassica nigra under intraspecific aboveground competition, which allowed us to elucidate how leaf removal by an insect herbivore impacts plant fitness through the changes in light climate and the interaction with dynamic plant responses to neighbours. Plants respond to damage by insect herbivores by eliciting the production of secondary metabolites that serve as a defensive mechanism to deter or hamper the growth of the attacking herbivore. Our current model does not incorporate this defensive mechanism as parameterisation on both the plant and herbivores were done using non-elicited plants. Plant defence elicitation has a potentially large effect on model predictions and is a logical next step in the development of our plant-plant-herbivore FSP model.

Plant competition for light and defence against herbivory are known to interact through the same signal that induces shade avoidance growth, R:FR, which also downregulates plant defences (Moreno et al., 2009; de Wit et al., 2013; Izaguirre et al., 2013; Ballaré, 2014; Campos et al., 2016; Cortes et al., 2016). This mechanism potentially allows the plant to optimise the allocation of its defences by prioritising leaves that are of high value to the plant (Izaguirre et al., 2013; de Vries et al., 2017). Plants are known to elicit a stronger defence response in younger leaves (Koricheva \& Barton, 2012), which is in line with our model predictions on the disproportionally high contribution of these leaves to plant competitiveness. This increase in secondary metabolism in young leaves is regulated by phytohormones such as cytokinins that also positively regulate primary metabolism (Giron et al., 2013; Brütting et al., 2017), hinting to a link between optimal resource and optimal defence allocation. When considering optimal defence allocation we should take into account that defence allocation patterns change over the course of plant development and that these whole-plant defence trajectories are much more variable than can be expected from the ontogenetic defence trajectory at the leaf level (Barton $\&$ Boege, 2017). This is in part due to changes in the need to prioritize growth, reproduction, competitive strength or defence over the plant's development (Boege \& Marquis, 2005). The optimal balance between growth- and defence-related traits depends on the current plant phenotype and on external selective forces such as herbivory and light competition. Future elucidation of whole-plant phytohormonal regulation of both primary and secondary metabolism is needed to fully understand the functioning of growth-defence trade-offs and put the predictions of the FSP modelling into perspective. Additionally, future developments of the FSP model to include regulation of primary and secondary metabolism can help elucidate the adaptive value of these regulatory mechanisms and how ontogenetic trajectories in optimal 
defence allocation affect growth-defence trade-offs.

Specialist and generalist herbivores not only differ in their feeding preferences (Cates, 1980), but also in their colonisation of a host plant. Specialist herbivores are known to preferentially oviposit on defended plants as they use plant defence chemicals as a host detection cue, whereas generalists often forgo these high defence phenotypes (Poelman et al., 2008a; Stam et al., 2014). The feedback between plant defences and the herbivore community colonising the plant can be an important driver of plant defence allocation (Poelman \& Kessler, 2016). The herbivore community experienced by a plant is the result of complex interaction between the behaviour and abundance of different herbivore species, as well as the phenotype and ecological context of the plant. Our results suggest that the way a given herbivore community impacts plant fitness depends on the composition of that herbivore community as well as the level of competition the plant is facing. These results emphasise the importance of considering the full range of dynamics in plant-plant-herbivore interactions when looking at growth-defence trade-offs (de Vries et al., 2017). A future challenge lies in elucidating the interaction of plant competition and herbivore community dynamics and how this interaction affect plant fitness. This challenge can be met by expanding our FSP model with plant defences and linking the plant defence phenotype to herbivore behavioural patterns on oviposition and feeding. These model developments add a level of dynamics to this already highly dynamic modelling approach and allow us to further elucidate the effect of dynamic plant-plantherbivore interactions on plant fitness.

\section{Acknowledgements}

This work was supported by the Netherlands Organization for Scientific Research (NWO) (project number: 823.01.004) and the European Research Council (ERC) under the European Union`s Horizon 2020 research and innovation programme (grant agreement nr: 677139 to EHP). We thank Marcel Dicke, Stefano Papazian and two anonymous reviewers for comments on an earlier version of the manuscript. 


\section{Supporting Information}

\section{Methods S1; Experimental supplementary}

Leaf angles - leaf angles were measured using ImageJ from pictures taken in the field in July 2014.

Branching angles - branching angles were measured using ImageJ from pictures taken in the field in July 2014.

Ear development - on the $14^{\text {th }}$ and $21^{\text {st }}$ of July 2014, we counted the number of pods on the main stem ear of the 9 selected plants in the three densities. The plants made on average $23.8 \pm 0.2$ pods (no difference between densities) over this 7 day period, which spanned 160.5 growing degree days (gdd) and translates to 6.74 gdd per pod. We counted the number of flowers per ear, which averaged at $11.4 \pm 0.15$ flowers per ear and translates to a flower life time of 77 gdd (11.4 flowers * 6.74 gdd per pod).

Photosynthetic capacity - in July 2014 and 2015 we selected one plant in the low density and used a LICOR (LI-6400XT, LI-COR Biosciences, Lincoln, Nebraska,USA) to measure the photosynthetic capacity of 9 mature leaves along the main stem. The photosynthetic capacity ranged from $32 \mu \mathrm{mol} \mathrm{m} \mathrm{m}^{-2} \mathrm{~s}^{-1}$ to $2.1 \mu \mathrm{mol} \mathrm{m}^{-2} \mathrm{~s}^{-1}$, which led us to use a base $\mathrm{A}_{\max }$ of $30 \mu \mathrm{mol}$ $\mathrm{m}^{-2} \mathrm{~s}^{-1}$ for out model.

Light quality $(R F R)$ - on the $1^{\text {st }}$ of July 2014 we measured the R:FR ratio in the three densities using a Skye SKR100/116 Fibre Optic Probe Measuring System (Skye Instruments Ltd, Powys, UK). We randomly selected 5 plants per density and measured the R:FR in four directions at 5 and $20 \mathrm{~cm}$ from the ground while pointing the sensor away from the selected plant.

\section{Methods S2; Model design supplementary}

A leaf can adjust its maximum rate of photosynthesis $\left(A_{\max }\right)$ based on the relative light interception (relPAR, dimentionless) and the maximum rate of photosynthesis of light grown leaves $\left(A_{\max 0}\right)$.

$$
A_{\max }=\left(A_{\max 0}\right)^{\text {relPAR*0.4 }}
$$

Gross photosynthesis rate $\left(A_{G}, \mu m o l \quad \mathrm{CO}_{2} \mathrm{~m}^{-2} \mathrm{~s}^{-1}\right)$ is calculated using the absorbed photosynthetically active radiation $(A P A R, \mu m o l)$, the initial slope of the light response curve $\left(\varepsilon, \mu \mathrm{mol} \mathrm{CO} \mathrm{Cmol}^{-1}\right)$ and the maximum rate of photosynthesis $\left(A_{\max } \mathrm{gCO}_{2} \mathrm{~m}^{-2} \mathrm{~d}^{-1}\right)$. 


$$
A_{G}=A_{\max } *\left(1-\exp \left(-\varepsilon * \frac{A P A R}{A_{\max }}\right)\right)
$$

Net photosynthesis $\left(A_{N}\right.$, $\left.\mu m o l \mathrm{CO}_{2} m^{-2} s^{1}\right)$ accounts for dark respiration $\left(R_{d}, \mu m o l \mathrm{CO}_{2}\right)$.

$$
A_{N}=A_{G}-R_{d}
$$

The assimilated $\mathrm{CO}_{2}$ is converted to produce sugars, a conversion parameter $(C)$ converts from $\mu \mathrm{mol} \mathrm{CO} 2$ to grams of glucose and seconds to hours, which is then multiplied by the daylength (hours).

$$
\frac{d \text { Sugar }}{d t}=C * \text { daylength } * A_{N}
$$

The assimilated carbon is distributed over the plant architecture to facilitate plant growth. The model distributes assimilates over the plant architecture by using a centralised carbon pool $(S$, gram sugar). Each growing organ will have a demand for assimilates expressed as a sink strength (Sink, g sugar), that includes assimilates needed to achieve potential growth

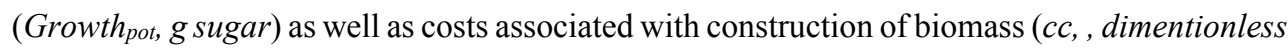
$>1)$ and respiration ( $r m$, dimentionless):

$$
\text { Sink }=\text { Growth }_{\text {pot }} * c c+\text { biomass } * r m
$$

Potential growth is calculated differently for different types of organs, depending on their geometrical and biological properties. The change in leaf length $(d L / d t, m)$ is calculated using the Yin functions for sigmoid growth (Yin 2003), where $t_{\mathrm{e}}$ represents the growth duration ( $g d d$ ), $\mathrm{t}_{\mathrm{m}}$ the point at which growth is maximal $(g d d), \mathrm{cm}$ is the growth at age $\mathrm{t}_{\mathrm{m}} . \mathrm{W}_{\max }$ is the potential size of the organ $(m)$ and $t$ is the organ age $(g d d)$.

$$
\begin{gathered}
\frac{d L}{d t}=c_{m}\left(\left(\frac{t_{e}-t}{t_{e}-t_{m}}\right)\left(\frac{t}{t_{m}}\right)^{\frac{t_{m}}{t_{e}-t_{m}}}\right) \\
c m=w_{\max }\left(\left(\frac{2 * t_{e}-t_{m}}{t_{e}\left(t_{e}-t_{m}\right)}\right)\left(\frac{t_{m}}{t_{e}}\right)^{\frac{t_{m}}{t_{e}-t_{m}}}\right)
\end{gathered}
$$

The value of $w_{\max }$ is based on empirical data of leaf length-rank profiles $\left(w_{\max 0}, m\right)$ and density effects. The lowest of the local or systemic R:FR signal $(r f r)$ and a number of parameters such as a leaf growth inhibition parameter $(L L r f r=0.4)$ calculate the eventual value of $w_{\max }$. 


$$
w_{\max }=w_{\max 0} *\left(1-\frac{1-L L r f r}{1+\exp (s *(r f r-m))}\right)
$$

Potential growth is calculated using a width:length ratio parameter $\left(r_{w l}\right)$ based on the leafs R:FR signal, the current and potential new leaf length $\left(L_{0}\right.$ and $\left.L_{1}, m\right)$, the current and potential new leaf width $\left(W_{0}\right.$ and $\left.W_{l}, m\right)$, a leaf shape conversion factor $\left(L_{\text {area }}\right)$ and the leaf mass per unit area $\left(L M A, g^{-2}\right)$.

$$
\begin{gathered}
L_{1}=L_{0}+\frac{d L}{d t} \\
W_{1}=\max \left(W_{0}, r_{w l} * L_{1}\right) \\
\text { Growt }_{\text {pot }}=\left(L_{1} * W_{1}-L_{0} * W_{0}\right) * L_{\text {area }} * L M A \\
r_{w l}=W L_{\max }-\frac{W L_{\text {max }}-W L_{\text {min }}}{1+\exp (s *(r f r-m))}
\end{gathered}
$$

Carbon allocation $(C A, g)$ is proportional to the sum of sinks (ss, $g$ sugar). This ensures that in carbon limiting conditions all growing organs have their growth reduced with an equal percentage.

$$
C A=\min \left(\operatorname{Sink}, S * \frac{\operatorname{Sink}}{S S}\right)
$$

The amount of carbon available for growth (CFlux, g sugar) then has to account for construction costs and maintenance respiration.

$$
\text { CFlux }=\frac{\text { CA-biomass } * r m}{c c}
$$

The carbon allocated to the organ leads to a change in biomass, which will translate in a change in organ size.

$$
\begin{gathered}
\frac{d \text { biomass }}{d t}=\text { CFlux } \\
\text { Area }=\text { biomass } / \text { LMA } \\
W_{\text {new }}=\sqrt{r_{w l} * \frac{\text { Area }}{L_{\text {area }}}}
\end{gathered}
$$




$$
L_{n e w}=\frac{W}{r_{w l}}
$$

However, since the width:length ratio is variable depending on light conditions we must account for the fact that leaves cannot become shorter or narrower.

$$
\begin{gathered}
W=\frac{\text { Area }}{L_{\text {area }}} * \frac{1}{L} \text { if } L_{\text {new }}<L \\
L=\frac{\text { Area }}{L_{\text {area }}} * \frac{1}{W} \text { if } W_{\text {new }}<W
\end{gathered}
$$

Internode growth is described using two independent mechanisms; internode length growth, which is affected by local rfr conditions, and internode radial growth, which is described using a pipe model. Internode length is described using the Yin function, with potential length (wmax, $g$ ) being calculated using the potential size in low densities $\left(w_{\max 0}, g\right)$ multiplied by an elongation parameter (Elong, dimentionless $>1$ ) dependent on local rfr signalling.

$$
w \max =w_{\max 0} * \text { Elong } \quad \text { if } \quad r f r<\text { threshold }
$$

The pipe model assumes that the cross-sectional surface area of an internode equals the sum of the cross-sectional surface area of its daughter internodes. The new radius of and internode $\left(r_{l}\right.$, $m$ ) thus depends on the radii of its daughter internodes $\left(r_{a}\right.$ and $\left.r_{b}, m\right)$ as follows;

$$
r_{1}=\sqrt{\frac{\pi r_{a}^{2}+\pi r_{b}^{2}}{\pi}}
$$

The potential growth of the internode is the difference between current and new volume multiplied by the internode tissue density $\left(T D_{I}, \mathrm{~g} \mathrm{~m}^{-3}\right)$.

$$
\text { Growth }_{\text {pot }}=\left(L_{1} * 2 * \pi * r_{1}-L_{0} * 2 * \pi * r_{0}\right) * T D_{I}
$$

The potential growth is translated to a sink strength and carbon flux in the same way as happens with the leaves. The allocated assimilates then lead to a change in internode size proportional to the ratio between the assimilates available for growth and the demand for those assimilates.

$$
\begin{aligned}
& \frac{\text { dlength }}{d t}=\left(L_{1}-L_{0}\right) * \frac{\text { CA }- \text { biomass } * r m}{\text { Sink }- \text { biomass } * r m} \\
& \frac{\text { dradius }}{d t}=\left(r_{1}-r_{0}\right) * \frac{C A-\text { biomass } * r m}{\text { Sink }- \text { biomass } * r m}
\end{aligned}
$$


Axillary meristems can grow and develop similar to the apical shoot meristem to form a branch. Branch initiation and abortion is simulated using cues that correspond to apical dominance, assimilate availability and light quality. Apical dominance is represented as a period of time after initiation of an axillary meristem during which it is not allowed to grow out into a branch (age $>$ MeristemDev), or when the dominance of the apical meristem is lifted because it becomes generative. Additionally, a bud needs enough sugar (SugarStatus $>$ SugarThreshold) to break and develop into a branch. Detection of neighbouring plants through an R:FR signal (see shade avoidance) or senescence or removal of the maternal leaf can abort a branch between the stage where it has formed three leaves (personal observation) up to when the axillary meristem becomes generative.

The potential growth of an ear is determined by the flower appearance rate $(6.7 \mathrm{gdd}$ per flower), the time it takes a flower to mature into a pod (77 gdd), as well as the potential seed mass of a pod. An ear can make a maximum of 80 pods, which contain 8 seeds with a potential weight of $8.37 \cdot 10^{-5} \mathrm{~g}$ per seed. 


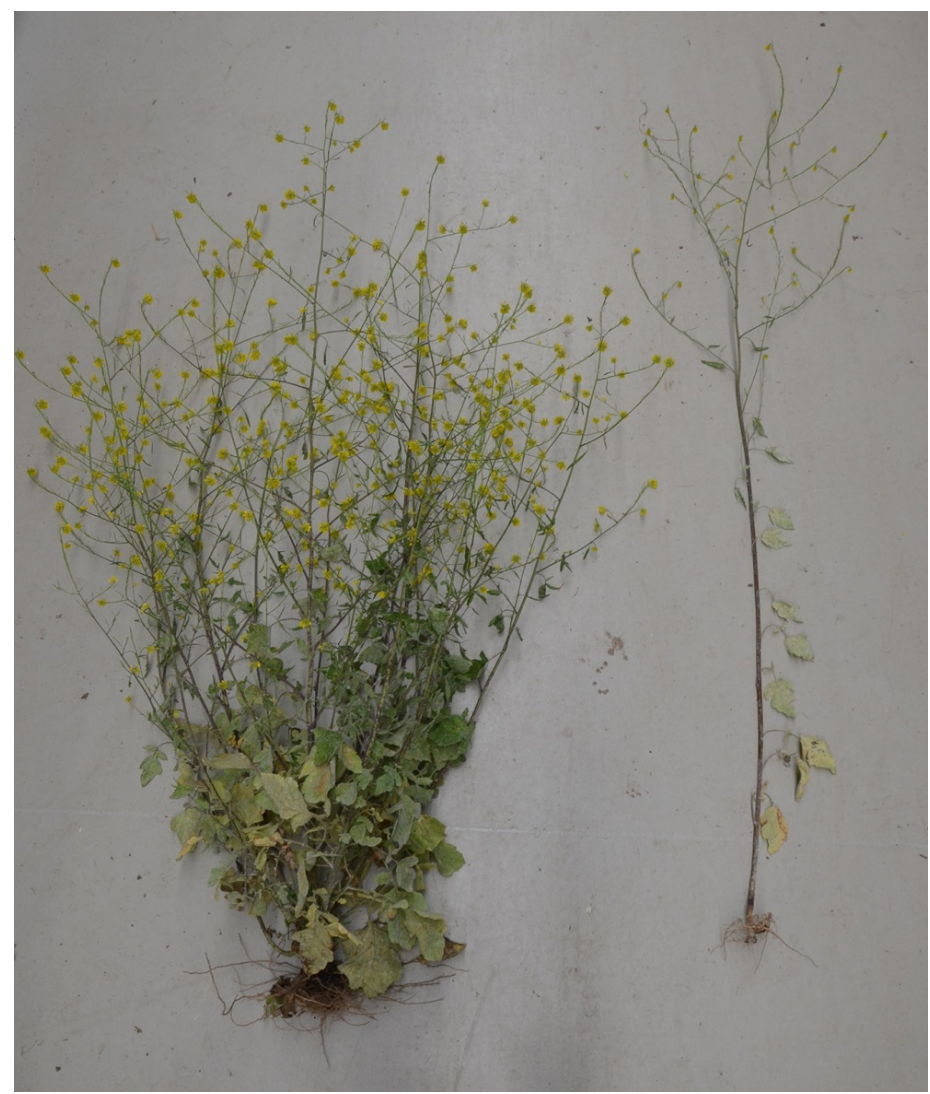

Figure S3.1. Field plants grown in a low density of $1 \mathrm{plant} / \mathrm{m}^{2}$ (left) and high density of 25 plants $/ \mathrm{m}^{2}$ (right) after 124 days of growth. 


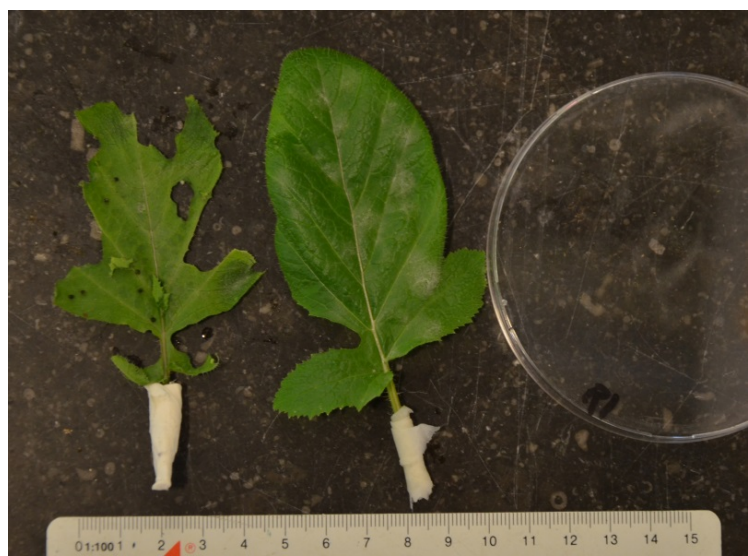

Figure S3.2. Two leaves used in the caterpillar greenhouse experiment, one after two days of caterpillar feeding (left) and one fresh leaf (right).

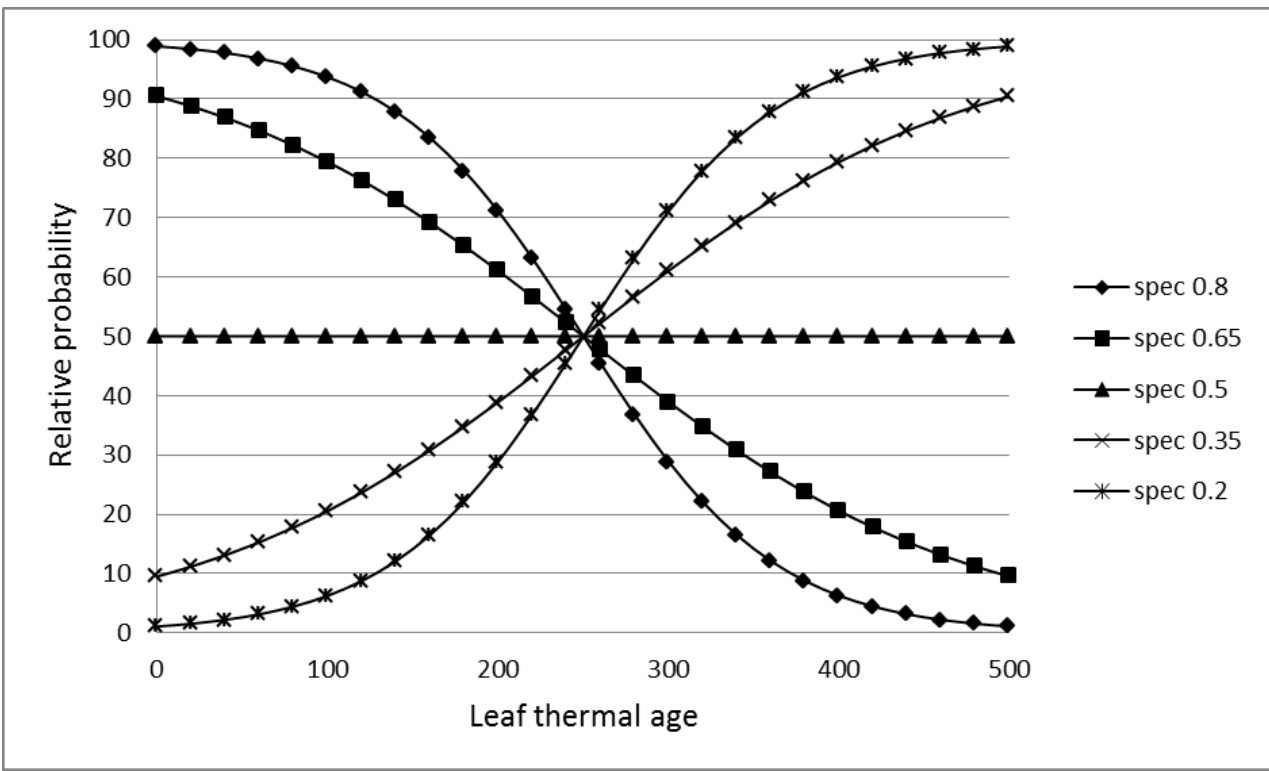

Figure S3.3. The relative probability a leaf is selected for feeding by a herbivore of a given level of specialisation $(h)$ as a function of the leafs age in degree days. A high $h$ parameter denotes a high level of herbivore specialisation and a high preference for young leaves, while a low spec parameter denotes a generalist herbivore with a feeding preference for older leaves. 
Low density

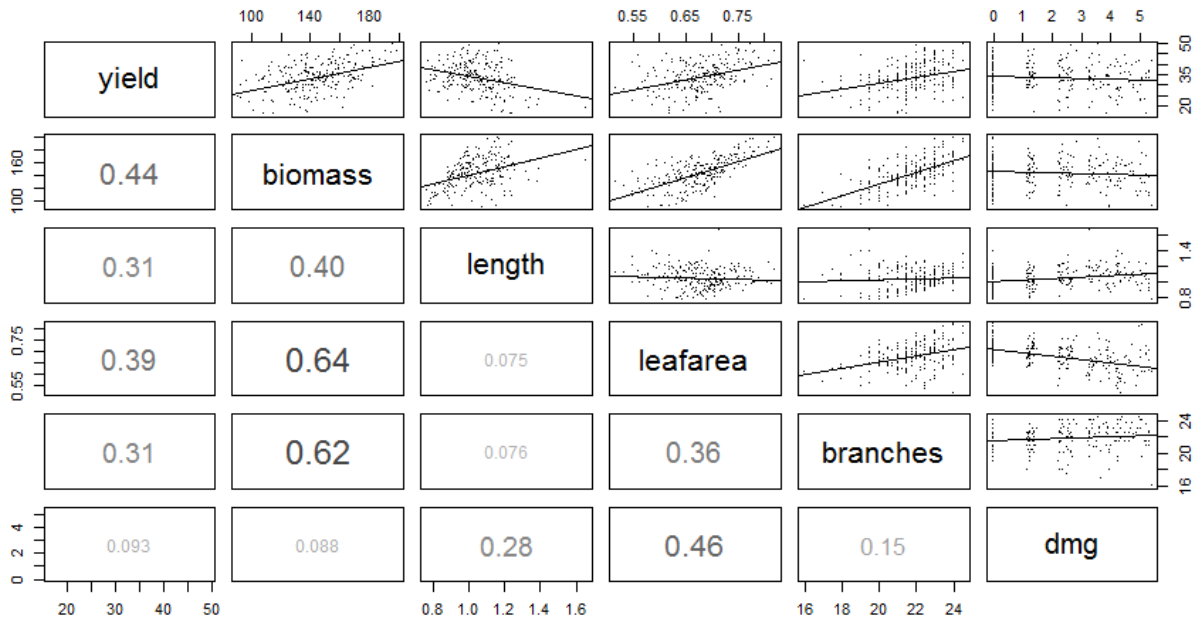

High density

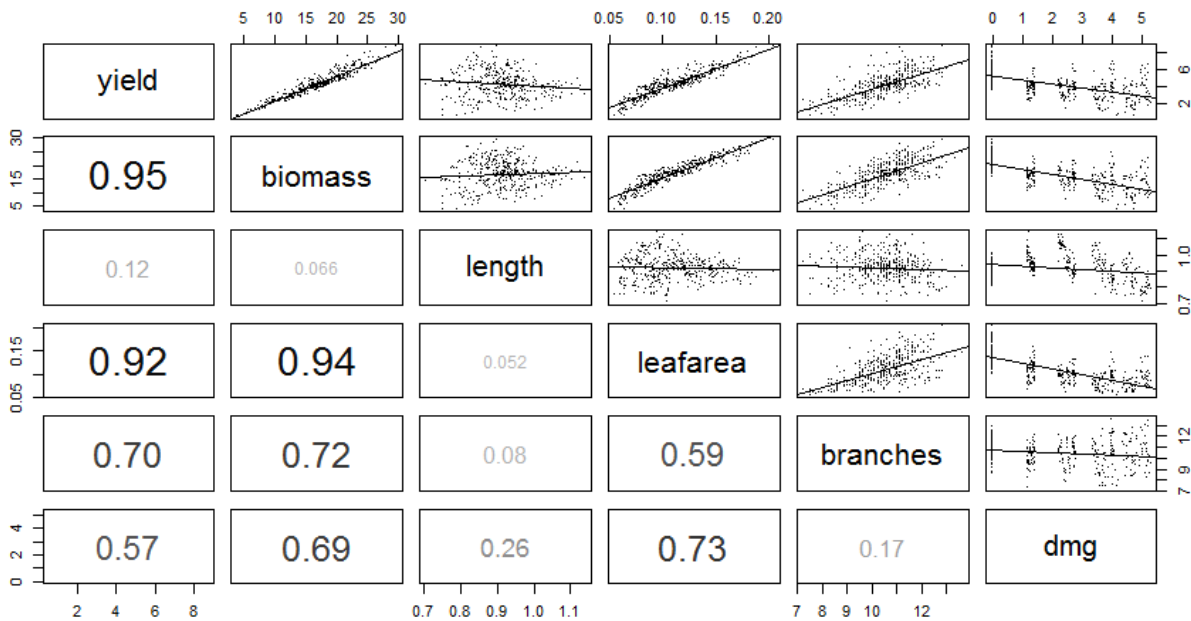

Figure S3.4. Correlations between six plant variables of an increasing level of integration within the modelling structure: herbivore damage (dmg), the number of branches (branches), max leaf area (leafarea), plant height (length), plant biomass (biomass), plant yield (yield). Regression lines are drawn through the point clouds plotted in the top panels. Corresponding $\mathrm{R}^{2}$ values are plotted in the bottom panels and show how much of the variation in the variable of a higher level of integration is explained by the variable of the lower level of integration. 


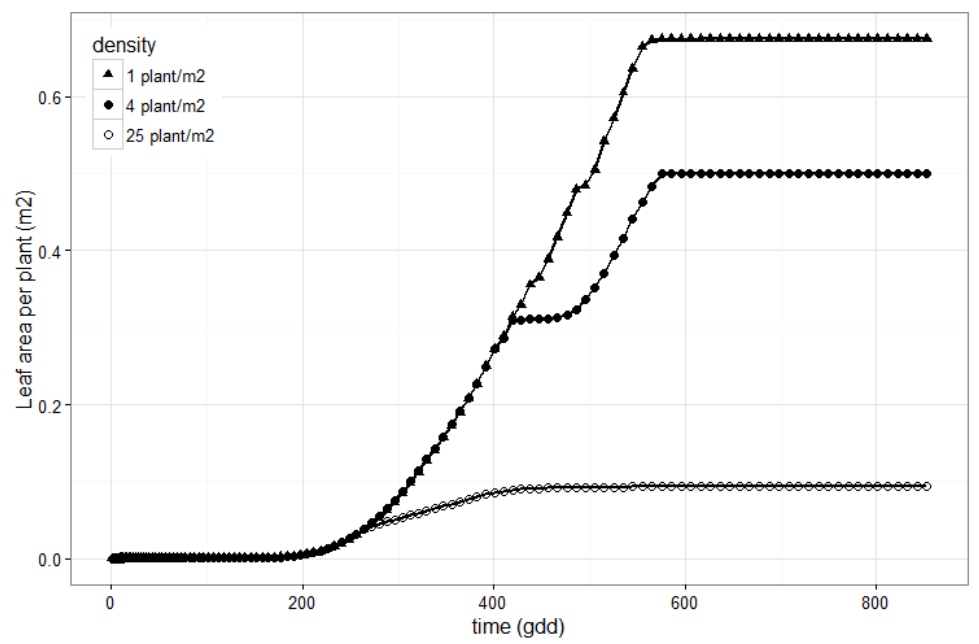

Figure S3.5. Simulated leaf area per plant $\left(\mathrm{m}^{2}\right)$ over time (gdd) in three densities $\left(1,4\right.$ and 25 plants $\left./ \mathrm{m}^{2}\right)$.

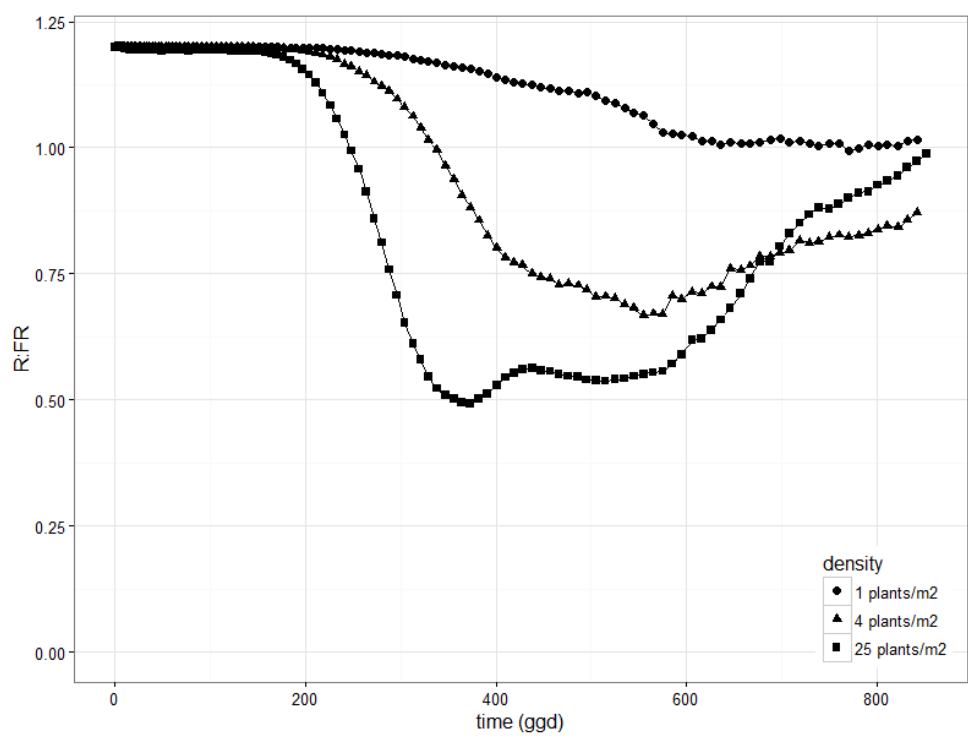

Figure S3.6. Simulated R:FR ratio over time (gdd) in three densities $(1,4$ and 25 plants $/ \mathrm{m}^{2}$ ). 


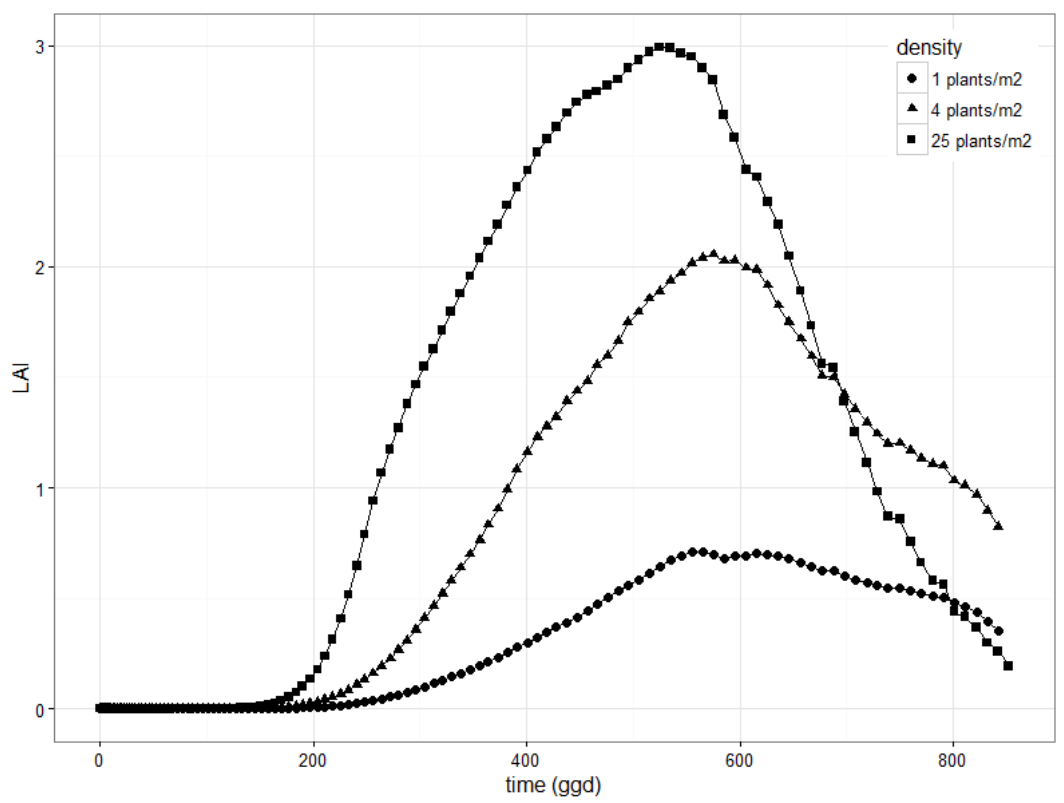

Figure S3.7. Simulated leaf area index (LAI) over time (gdd) in three densities $\left(1,4\right.$ and 25 plants $\left./ \mathrm{m}^{2}\right)$. 



\section{Chapter 4}

\section{Ecological interactions shape the adaptive value of plant defence:} herbivore attack versus competition for light

\footnotetext{
Jorad de Vries ${ }^{1,2}$, Jochem B. Evers ${ }^{2}$, Marcel Dicke ${ }^{1}$ and Erik H. Poelman ${ }^{1}$

${ }^{1}$ Wageningen University, Laboratory of Entomology, Wageningen, The Netherlands

${ }^{2}$ Wageningen University, Centre for Crop System Analysis, Wageningen, The Netherlands
} 


\section{Chapter 4}

\section{Abstract}

Plants defend themselves against diverse communities of herbivorous insects. This requires an investment of limited resources, for which plants also compete with neighbours. The consequences of an investment in defence are determined by the metabolic costs of defence as well as indirect or ecological costs through interactions with other organisms. These ecological costs have a potentially strong impact on the evolution of defensive traits, but have proven to be difficult to quantify.

We aimed to quantify the relative impact of the direct and indirect or ecological costs and benefits of an investment in plant defence in relation to herbivory and inter-genotypic competition for light. Additionally, we evaluated how the benefits of plant defence balance its costs in the context of herbivory and inter-genotypic competition.

To this end, we utilised a functional-structural plant (FSP) model of Brassica nigra that simulates plant growth and development, morphogenesis, herbivory and plant defence. In the model, a simulated investment in defences affected plant growth by competing with other plant organs for resources and affected the level and distribution of herbivore damage.

Our results show that the ecological costs of inter-genotypic competition for light are highly detrimental to the fitness of defended plants, as it amplifies the size difference between defended and undefended plants. This leads to herbivore damage counteracting the effects of inter-genotypic competition under the assumption that herbivore damage scales with plant size. Additionally, we show that plant defence relies on reducing herbivore damage rather than the dispersion of herbivore damage, which is only beneficial under high levels of herbivore damage.

We conclude that the adaptive value of plant defence is highly dependent on ecological interactions and is predominantly determined by the outcome of competition for light. 


\section{Introduction}

In natural settings, plants are part of complex communities of herbivores and neighbouring plants that shape the adaptive value of growth and defence-related traits (Lankau \& Strauss, 2008; Poelman, 2015; de Vries et al., 2017). The interactions within these communities give rise to trade-offs in growth and defence that maximise fitness while responding to a variable environment (Züst \& Agrawal, 2017). Direct competition between two plant traits over the limiting pool of an individual's internal resources is perhaps the most commonly considered driver of the trade-offs observed between a plant's ability to defend against herbivorous insects and the mechanisms that allow the plant to compete for light with neighbouring plants (Herms \& Mattson, 1992; Züst et al., 2011; Ballaré, 2014; Züst \& Agrawal, 2017). It is apparent that defensive mechanisms bring substantial metabolic costs that include costs of the machinery for the synthesis, modification, transport, maintenance and storage of the plant secondary metabolites (Gershenzon, 1994; Bekaert et al., 2012). However, these direct costs do not always result in a loss of fitness and might only be relevant under certain ecological settings such as resource limitation, competition for resources, the presence of herbivores and pathogens or when the plant's mutualists are affected (Heil \& Baldwin, 2002; Koricheva, 2002; Strauss et al., 2002; Dicke \& Hilker, 2003; Cipollini et al., 2014). The expression of costs through interactions between the plant and biotic or abiotic conditions in its environment can be defined as ecological costs. These ecological costs can have a substantial impact on plant fitness and are, therefore, important drivers of evolution (Heil, 2002; Dicke \& Hilker, 2003). However, identifying and quantifying the ecological costs associated with plant defence is complicated by the myriad of possible effects of the plant defence trait on other community members (Heil \& Baldwin, 2002; Stam et al., 2014). Even when isolating a single interaction in an experimental setup, discriminating ecological costs from metabolic costs is often complicated due to the complex and interwoven nature of the physiological and ecological mechanisms driving plant-plant-herbivore interactions (de Vries et al., 2017).

The interaction between physiological and ecological costs is apparent in the synthesis and allocation of plant chemical defences against insect herbivores. Plants are known to exhibit a stronger defence response in younger leaves (Koricheva \& Barton, 2012), which follows the allocation of key nutrients such as nitrogen towards plant parts that are most favourably positioned relative to resource gradients (McKey, 1974; Hirose et al., 1987; Hirose, 2005; Hikosaka et al., 2016). This local pattern of defence expression offers potential benefits to the 
plant if it results in dispersing herbivore damage within the plant and away from most valuable tissues (Cipollini et al., 2014). However, the response of a herbivore to a plant's defence expression depends on that herbivore's sensitivity to plant-taxon-specific secondary metabolites, which differs greatly between herbivore species (Bennett \& Wallsgrove, 1994). Those that have specialised on a particular host-plant taxon are more resistant to the defensive mechanisms adopted by that taxon, making them less susceptible to the toxic or digestive reducing function of the defensive secondary metabolite. This differentiation in host-plant specialisation makes the composition of the insect community attacking the plant a strong determinant of the benefits the plant receives for its investment in defence. Heterogeneity in the distribution of nutritional and defensive value of leaves in the canopy is expected to result in different herbivore distribution patterns depending on the level of specialisation of the members of the herbivore community. The increased resistance of specialised herbivores to defensive compounds allows them to feed from the more nutritious, yet better defended parts of their host plant such as young leaves, buds and seeds (Feeny, 1976; Cates, 1980; Schoonhoven et al., 2005). Conversely, the elevated levels of defence in these important plant parts deter the more generalist herbivore species that are then forced to feed on less defended but also less nutritional plant tissues such as mature leaves (Feeny, 1976; Cates, 1980; Schoonhoven et al., 2005). The distribution pattern of the herbivore community resulting from these differences in herbivore sensitivity to plant defence in turn have a strong impact on plant fitness. This is especially true in a competitive environment where the removal of young leaves decreases plant competitive ability and consequentially fitness more than the removal of mature leaves (de Vries et al., 2018b). Therefore, we expect the adaptive value of plant defence to be more dependent on the ecological costs through the effect on the plant's competitive ability and herbivore damage than on the metabolic costs of these defences (Agrawal, 2000; Heil et al., 2000; Van Dam \& Baldwin, 2001).

In this paper we aim to (i) quantify the direct costs of plant defence as well as the ecological costs imposed by herbivore damage and inter-genotypic competition for light. We expect the ecological costs imposed by herbivore damage and inter-genotypic competition for light to exceed the direct costs of plant defence, resulting in a stronger impact on plant fitness. We also aim to (ii) quantify the direct benefits of plant defence through a reduction or redistribution of herbivore damage, as well as the ecological effect of this benefit under intergenotypic competition for light. Finally (iii), we evaluate the level of benefits required to outweigh the direct and ecological costs of plant defence, at which point plant defence becomes 


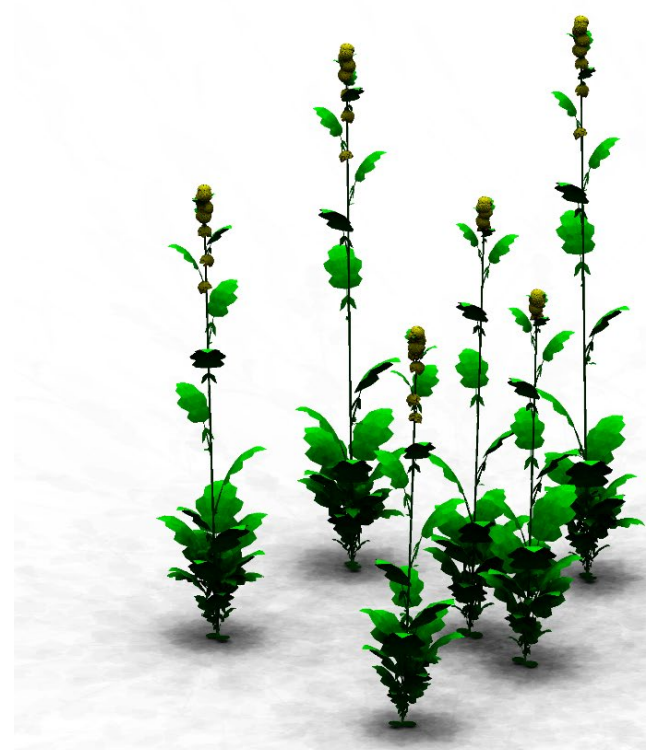

Undamaged

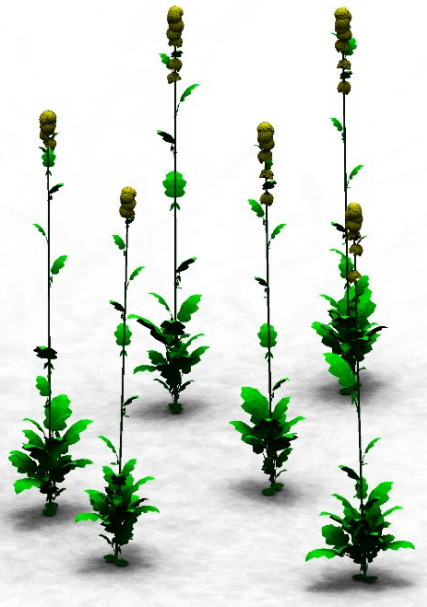

Damaged

Figure 4.1 Visual representation of the FSP model, showing undamaged and damaged plants. Herbivore damage reduces leaf area, which affects canopy structure and subsequent light climate. For visualisation purposes, the plant density in the Figure is lower than the plant density used in the simulations.

adaptive to the plant. We expect plant defence to be especially effective if it results in dispersion as well as reduction of herbivore damage. Here, we study the interaction between defence investment, competition for light and herbivory using a modelling approach called functionalstructural plant (FSP) modelling (Vos et al., 2010; Evers, 2016). This three-dimensionally explicit modelling approach allows for the simulation of individual plants that grow and compete for resources with neighbouring plants. FSP modelling has proven to be a powerful tool to simulate plant competition for light and the associated effects on source-sink dynamics (Evers et al., 2010; Evers \& Bastiaans, 2016) and architectural responses (Evers et al., 2007; Zhu et al., 2015; Bongers et al., 2018).

\section{Methods}

\section{Model description}

To elucidate the interaction between plant competition for light, herbivory and plant defence 
we expanded the plant-herbivore FSP model of Brassica nigra described previously (de Vries et al., 2018b), which is developed in the modelling platform GroIMP (Hemmerling et al., 2008). This model has been parameterised and validated using detailed field measurements on Brassica nigra architecture, growth and development, biomass and seed yield. In summary, this three-dimensional model mechanistically simulates aboveground plant growth and competition for light through source-sink dynamics in carbon assimilation and allocation and light-driven mediation of plant architecture (for a detailed model description see de Vries et al. (2018b)). We expanded the existing model by adding a plant defence module that impacts herbivore damage and acts as a carbon sink, interacting with plant growth through the source-sink dynamics of the plant. These additions are described in detail below.

In the model, we simplified plant defence to an on/off mechanism where defended plants allocate a fixed percentage (D) of the assimilates produced with photosynthesis $\left(A_{\text {total }}\right.$, in $\left.g\right)$ towards the biosynthesis and maintenance of plant defence. The remaining assimilates $\left(\mathrm{A}_{\text {growth }}\right.$, in $\mathrm{g}$ ) are allocated to the maintenance of standing biomass and the growth of new biomass.

$$
A_{\text {growth }}=\left(1-\frac{D}{100}\right) * A_{\text {total }}
$$

In the model, herbivory is represented by a rate of damage to an individual leaf over time as a function of leaf area (Feeny, 1976; Johnson \& Agrawal, 2005; Schoonhoven et al., 2005), capturing the damage done by a community of chewing herbivores. This rate of herbivore damage reduces both the current size of a leaf, representing the actual removal of area, as well as the potential size of a leaf, limiting the further growth of a damaged leaf (see Figure 4.1 for the results of herbivore damage on plant architecture). The rate of herbivore damage is fixed on the plant level, assuming no dispersal of herbivores between plants as a result of a plant's defence investment. The herbivore damage suffered by a plant is simulated using a sigmoidal function that describes the total amount and distribution of herbivore damage within the plant (eq. 4.2). The rate of damage by herbivory on the leaf level per growing degree day (GDD) (dmg, g leaf biomass $\mathrm{GDD}^{-1}$ ) is calculated using the leaf biomass (b, in $\mathrm{g}$ ), the relative leaf rank ( $\mathrm{r}$ ), the herbivore distribution ( $h$, value from 0 to 1 , see Figure $4.3 \& 4.4$ ), the base rate of leaf removal (c, fraction of leaf biomass $\mathrm{GDD}^{-1}$ ) and the damage reduction by plant defence $(\mathrm{d}, \%)$ : 


$$
d m g=\frac{c *\left(1-\frac{d}{100}\right) * b}{1+\exp (10 *(h-(1-h)) *(r-0.5))}
$$

Leaf rank was used to number the leaves and is an indicator of the leaf's position along the main stem. The relative rank of a leaf (r) calculated using the absolute rank of the leaf $\left(\mathrm{r}_{\mathrm{a}}\right)$, the highest $\left(r_{\max }\right)$ and lowest $\left(r_{\min }\right)$ ranked leaf on the same plant:

$$
r=\frac{r_{a}-r_{\min }}{r_{\max }-r_{\min }}
$$

Equation 2 was simplified for undefended plants where the damage reduction by plant defence equals 0 and the herbivore distribution parameter equals 0.2 , which represents herbivore preference for young leaves in the absence of defence (Cates, 1980; Schoonhoven et al., 2005):

$$
d m g=\frac{c * b}{1+\exp (-6 *(r-0.5))}
$$

This function allows for simulation of different scenarios of costs and benefits of plant defence, which are described in the next section. Plant defence affects the total amount of herbivore damage, describing the reduction of herbivore growth and the subsequent reduction of herbivore damage. The model does not explicitly describe defence expression at the leaf level, but implicitly assumes that the presence of defence can affect the distribution of herbivore damage (depending on the scenario), reflecting how different herbivore species in the herbivore community might respond to plant defence. In undefended plants, we assume a distribution of herbivore damage that favours young leaves due to these being more nutritional than older leaves (which is not explicitly represented in the model). In defended plants, we expect a dispersal of generalist herbivore species towards older leaves (Cates, 1980; Schoonhoven et al., 2005), which is modelled through a shift in the shape of the sigmoidal function described in eq. 4.2 by increasing the value of $h$.

\section{General simulation setup}

Plants were simulated in plots of 16 plants at a density of 100 plants $\mathrm{m}^{-2}$. This small plot was cloned 625 times to construct a large field with 10,000 plants for light model calculations, where every individual plant is represented 625 times at regular intervals. The light interception of the 
625 clones of each individual plant was averaged every time step to get the light interception of each individual plant, this method evens out border effects that would otherwise be prevalent in a small plot. Simulations ran from the $31^{\text {st }}$ of March to the $2^{\text {nd }}$ of August (124 days), with average daily temperature, average daily insolation and solar angle typical for the Netherlands at a latitude of 52 degrees (Evers et al., 2010; Zhu et al., 2015).

\section{Scenarios: Direct and ecological costs of plant defence (i)}

To quantify the impact of plant defence on plant fitness we imposed five levels of photosynthetic costs on defending plants $(\mathrm{D}=5,10,15,20$ and $25 \%$ of assimilates produced by photosynthesis in eq. 4.1), which spans the range of direct costs found in a multitude of plant species (Strauss et al., 2002; Bekaert et al., 2012).

The treatments were set up as follows:

1) We first simulated mono-stands of undefended, undamaged plants that act as a control, providing a baseline measure of plant fitness to which the following treatments are compared.

2) To quantify the direct (metabolic) costs of plant defences we simulated mono-stands of defended plants in the absence of herbivore damage.

3) To quantify the ecological costs of herbivore damage, we simulated mono-stands of plants that invested in defence and suffered low $(\mathrm{c}=0.005)$ or high $(\mathrm{c}=0.01)$ herbivore damage, without receiving benefits for their investment in defence $(h=0.2, d=0$, eq. 4.2).

4) To quantify the ecological costs imposed by inter-genotypic competition, we simulated mixed stands of defended and undefended plants in the absence of herbivore damage.

5) To quantify the combined effect of herbivore damage and inter-genotypic competition we then simulated mixed stands of defended and undefended plants in which all plants suffered low $(\mathrm{c}=0.005)$ or high $(\mathrm{c}=0.01)$ herbivore damage, without the defending plants receiving a benefit for their investment in defence $(h=0.2, d=0$, eq. 4.2).

\section{Scenarios: Direct and ecological benefits of plant defence (ii)}

To quantify the direct and ecological benefits of plant defence we simulated defended plants that did not pay the metabolic costs associated to this defence investment. These plants were simulated in mono-stands to determine the direct benefits of plant defence and in mixtures to determine the ecological benefits of plant defence. Both the mono-stands and mixtures were 
subjected to low $(\mathrm{c}=0.005)$ or high $(\mathrm{c}=0.01)$ herbivore damage and we simulated six levels of herbivore damage reduction for defended plants $(d=0,10,20,30,40,50 \%$, eq. 4.2$)$ and three herbivore distributions for defended plants $(h=0.2,0.5,0.8$, eq. 4.2$)$ in a full factorial design.

\section{Scenarios: Costs and benefits of plant defence (iii)}

To quantify when the benefits of plant defence outweigh the total costs of plant defence, we simulated mixtures of defended and undefended plants where all plants suffered low $(c=0.005)$ or high $(\mathrm{c}=0.01)$ herbivore damage and where the defended plants allocated $15 \%$ of the assimilates produced by photosynthesis to defence (Bekaert et al., 2012). We assumed that plant defence can reduce damage as well as change the distribution of damage within the plant from younger towards older leaves. To quantify the importance of reducing the total amount of damage and the distribution of damage, we simulated six levels of herbivore damage reduction for defended plants $(d=0,10,20,30,40,50 \%$, eq. 4.2) and tree herbivore distributions for defended plants $(\mathrm{h}=0.2,0.5,0.8$, eq. 4.2$)$ in a full factorial design. The undefended plants suffered the baseline level of herbivore damage $(d=0)$, the distribution of which was skewed towards young leaves $(\mathrm{h}=0.2)$.

\section{Output}

The simulated seed yield (e.g. the investment of biomass into seeds) per plant was used as a proxy for plant fitness. Seed yield is an emergent property of the model that arises from the interaction between source-sink dynamics, herbivore damage and competition for light. We use one of three types of output to show our results:

1. To quantify the direct costs of plant defences, we simulated mono-stands of undefended, undamaged control plants to act as a baseline for plant fitness. To quantify the ecological costs of plant defences we simulated mixtures of defended and undefended plants, using the undefended plants to act as a baseline for the fitness of the defended plants. We calculate the costs imposed by a given treatment (C, \% yield decrease) by comparing the yield of the treatment (Yield ${ }_{\mathrm{T}}$ ) to the baseline yield of the control plants $\left(\right.$ Yield $\left._{C}\right)$.

$$
C=\left(1-\frac{\text { Yield }_{T}}{\text { Yield }_{C}}\right) * 100
$$

2. To quantify the direct benefits of plant defences we simulated mono-stands of 
undefended plants facing low or high herbivore damage to act as a baseline for plant fitness. To quantify the ecological benefits of plant defences, we simulated mixtures of defended and undefended plants facing low or high herbivore damage, and used the undefended plants to act as a baseline for the fitness of the defended plants. We calculate the benefits provided by a given treatment (B, \% yield increase) by comparing the yield of the treatment $\left(\right.$ Yield $\left._{\mathrm{T}}\right)$ to the baseline yield of the control plants $\left(\right.$ Yield $\left._{C}\right)$.

$$
B=\left(\frac{\text { Yield }_{T}}{\text { Yield }_{C}}-1\right) * 100
$$

The model output was tested for significance at the $5 \%$ probability level by conducting an analysis of variance (ANOVA). Values reported in the text are shown as (mean \pm se unit) and error bars in graphs represent the standard error of the mean.

\section{Results}

Direct and ecological costs of plant defence (i)

The direct effect of investing in plant defence on plant fitness was proportional to the percentage of photosynthesis that was invested in defence (Figure 4.2a). Inter-genotypic competition with undefended plants had a disproportionately strong negative effect on the yield of defended plants in the absence of herbivory $(\mathrm{F}=64.9, \mathrm{P}<0.001$; Figure $4.2 \mathrm{a})$. The direct effect of herbivore damage decreased with an increasing investment in defence (difference between the solid/dotted line and the dashed line in Figure 4.2b,c, $\mathrm{F}=5.0, \mathrm{P}<0.05)$. This decrease in the effect of herbivory was caused by a reduction of plant size with an increasing investment into defence in conjunction with herbivore damage being proportional to leaf area. This mechanism also led to herbivore damage reducing the negative effect of inter-genotypic competition $(\mathrm{F}=27.6$, $\mathrm{P}<0.001$; Figure $4.2 \mathrm{~b}, \mathrm{c})$. Herbivore damage balanced the yield differences between defended and undefended plants that emerged from inter-genotypic competition, as competitively strong plants suffered more herbivore damage due to their larger size. Inter-genotypic competition still reduced the yield of defended plants when suffering low rates of herbivore damage $(\mathrm{F}=13.7$, $\mathrm{P}<0.001$; Figure $4.2 \mathrm{~b}$ ), but had no effect on the yield of defended plants under high rates of herbivore damage $(\mathrm{F}=0.9, \mathrm{P}=0.35$; Figure $4.2 \mathrm{c})$.

\section{Benefits of plant defence (ii)}

The direct benefits of plant defence, illustrated by a plant facing intra-genotypic competition, 


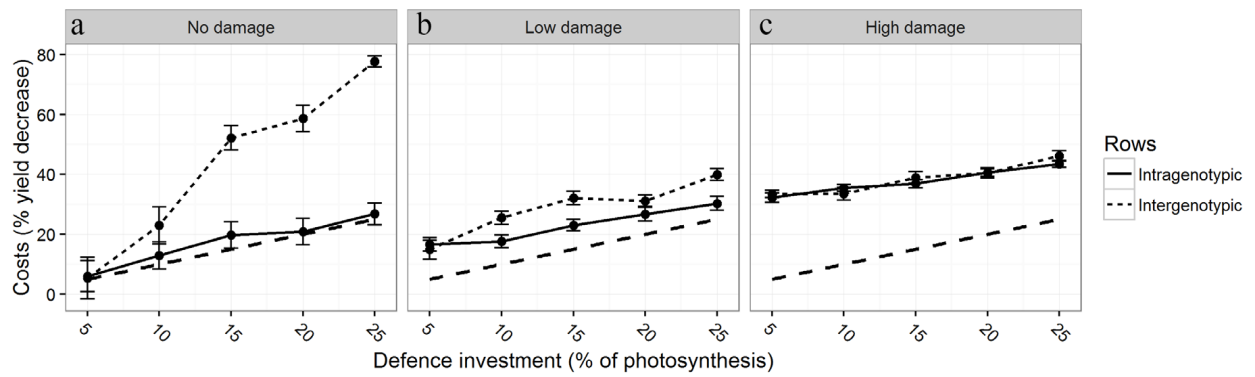

Figure 4.2 The costs (\% yield decrease, y-axis) imposed by five levels of defence investment ( $\%$ of photosynthesis, $\mathrm{D}$ in eq. 1 , $\mathrm{x}$-axis) for defended plants facing intra-genotypic competition with other plants of the same defence type (solid line) or inter-genotypic competition with undefended plants (dotted line) and either no (a), low (b) or high (c) herbivore damage. The dashed line represents the line where the yield decrease is proportional to the investment in plant defence. Error bars show standard error of the mean.

were apparent both when reducing $(\mathrm{F}=53.7, \mathrm{P}<0.001$; Figure $4.3 \mathrm{a}, \mathrm{b}, \mathrm{c})$ and redistributing $(\mathrm{F}=25.6, \mathrm{P}<0.001$; Figure 4.3a,b,c) herbivore damage, but the fitness benefits were far more substantial under high than under low levels of herbivore damage $(\mathrm{F}=323, \mathrm{P}<0.001$; Figure $4.3 \mathrm{a}, \mathrm{b}, \mathrm{c})$. In plants that faced low levels of herbivore damage and inter-genotypic competition with undefended plants, these direct benefits translated to an indirect benefit when reducing herbivore damage $(\mathrm{F}=148, \mathrm{P}<0.001$; Figure $4.3 \mathrm{~d}, \mathrm{e}, \mathrm{f})$, but not when redistributing herbivore damage $(\mathrm{F}=2.2, \mathrm{P}=0.11$; Figure $4.3 \mathrm{~d}, \mathrm{e}, \mathrm{f})$. Under high levels of herbivore damage, the direct benefits translated to an indirect benefit both when reducing herbivore damage $(F=210$, $\mathrm{P}<0.001$; Figure 4.3d,e,f), and when redistributing herbivore damage ( $\mathrm{F}=3.1, \mathrm{P}<0.05$; Figure $4.3 \mathrm{~d}, \mathrm{e}, \mathrm{f})$.

\section{Costs vs benefits of plant defence (iii)}

To quantify the level of benefits required to balance the investment costs of plant defence, we simulated mixed stands of defended and undefended plants in which plant defence changed the distribution and/or amount of herbivore damage, assuming a damage investment percentage of $15 \%$. Our results show that under low levels of herbivory, defended plants outcompeted their undefended neighbours, resulting in a positive net benefit, when their defence resulted in at least a $30 \%$ reduction in herbivore damage (Figure 4.4). Under high levels of herbivory, defended plants outcompeted their undefended neighbours, resulting in a positive net benefit, when their defence resulted in at least a 10\% reduction in herbivore damage (Figure 4.4). Except at a herbivore distribution skewed towards old leaves, where the defending plants always 


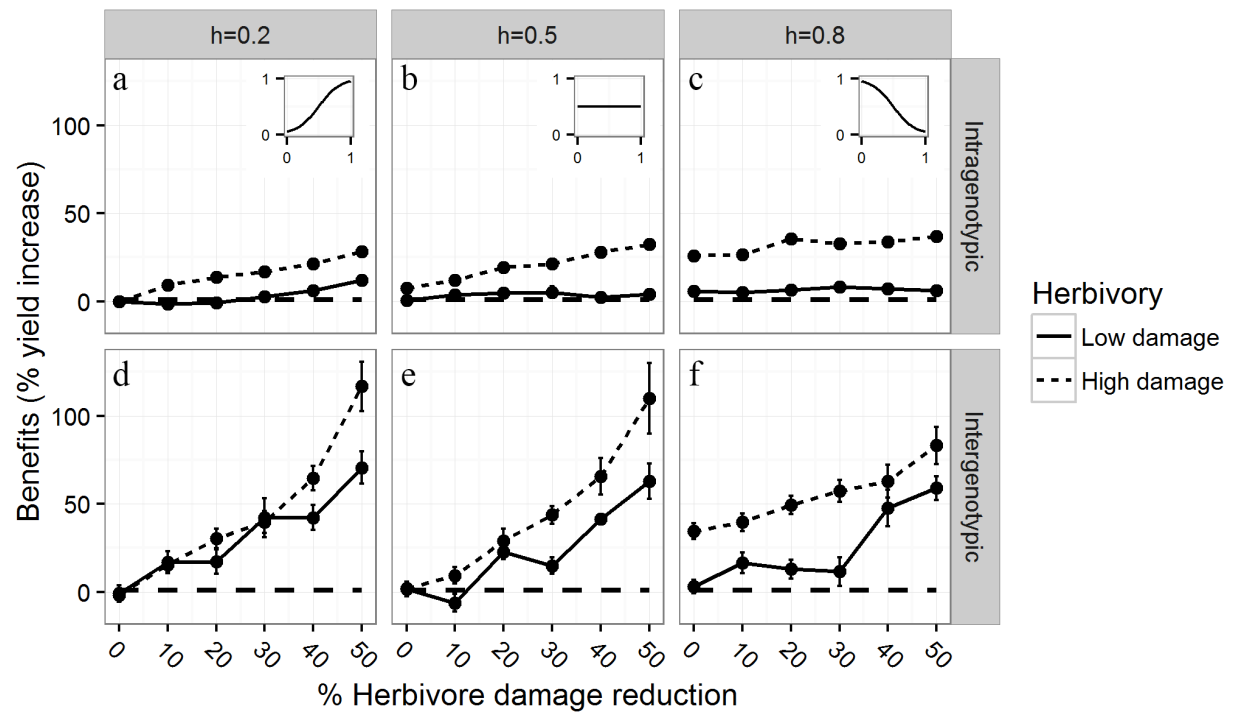

Figure 4.3. The benefits (\% yield increase, y-axis) as a result of a reduction in herbivore damage (x-axis) or a redistribution of herbivore damage (columns, $\mathrm{h}$ in eq 3 ) for defended plants that payed no costs for their defence investment and faced either intragenotypic competition with other defended plants $(a, b, c)$ or intergenotypic competition with undefended plants $(\mathrm{d}, \mathrm{e}, \mathrm{f})$. The dashed horizontal line represents the level above which the defended plants outcompete undefended plants. The subplots show the herbivore distribution (y-axis) as a function of relative leaf rank ( $\mathrm{x}$-axis, lowest leaf rank $=0$, highest leaf rank $=1)$ for the corresponding value of $h$. Error bars show standard error of the mean.

outcompeted their undefended neighbours $(\mathrm{h}=0.8$, Figure $4.4 \mathrm{e})$. These tipping points cannot be explained by the direct costs and benefits of defence as the fitness decrease as a result of a defence investment (15\%) was higher than the direct fitness benefit of reducing herbivore damage at the tipping points (low herbivory: $2.7-8.5 \%$ yield increase at $30 \%$ damage reduction; high herbivory: $9.2-12 \%$ yield increase at $10 \%$ damage reduction, see Figure 4.3 ). The differences in herbivore distributions did not lead to differences in plant fitness under low levels of herbivore damage ( $\mathrm{P}=0.43$, Figure 4.4), but could lead to an increase in plant fitness under high levels of herbivore damage $(\mathrm{F}=9.5, \mathrm{P}<0.005$, Figure 4.4). These differences in herbivore distribution did affect the plant, as shown by the final biomass of leaves, which was affected by the herbivore distribution such that the final leaf biomass was inversely correlated with herbivore damage distribution (Figure S4.1).

\section{Discussion}

We show that the indirect costs and benefits of plant defence through ecological interactions 


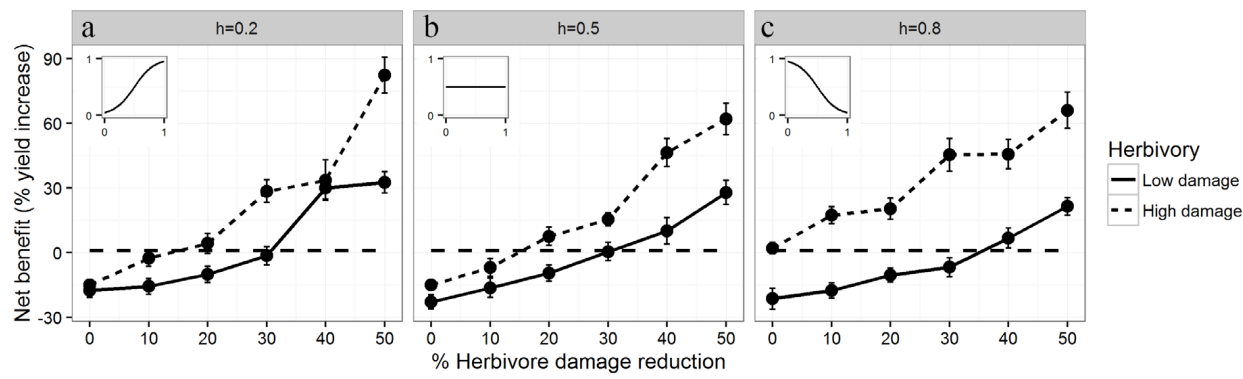

Figure 4.4 The net benefit (y-axis, \% yield increase) provided by a defence investment while competing with undefended plants competing in mixed stands. The defended plants benefitted from their defences by reducing herbivore damage (x-axis, $\%$ herbivore damage reduction, $\mathrm{d}$ in eq. 2) and/or changing the distribution of herbivore damage (panels, $h$ in eq 3 ). The dashed horizontal line represents the level above which the defended plants outcompete their undefended neighbours. The subplots show the herbivore distribution (y-axis) as a function of relative leaf rank ( $\mathrm{x}$-axis, lowest leaf rank $=0$, highest leaf rank $=1$ ) for the corresponding value of $h$. Error bars show standard error of the mean.

are more important than, and disproportionate to, the effects of direct costs and benefits of plant defence on plant fitness. Our results show that the direct costs of a defence investment were proportional to the size of the investment. However, the indirect effects through ecological interactions with herbivores and neighbouring competitors were highly context dependent and not proportional to the direct effect on plant fitness. Our results further show that the ecological costs of inter-genotypic competition had the strongest impact on plant fitness among the tested treatment (Figure 4.2d). The ecological costs of competition for light scaled disproportionately with the investment in plant defences, which was caused by the asymmetric nature of competition for light (e.g. stronger competitors taking a disproportionate share of resources (Weiner, 1990; Freckleton \& Watkinson, 2001)). The model assumed that herbivore damage scaled with leaf area, making it dependent on plant size (Feeny, 1976; Johnson \& Agrawal, 2005; Schoonhoven et al., 2005). This led to a decrease in the effect of herbivore damage on yield with an increasing investment in defence (difference between the solid/dotted lines and the dashed lines in Figure 4.2b,c), and a decrease in the negative effect of a defence investment under inter-genotypic competition due to herbivore damage (difference between the dotted lines in Figure 4.2a and Figure 4.2b,c). If we were to assume that herbivore damage did not scale with leaf area and remained constant regardless of plant size, herbivore damage would likely amplify the asymmetry of competition for light. In this case the amount of damage inflicted by the herbivores relative to plant size is higher for under-performing plants, giving them a further disadvantage compared to their over-performing neighbours. 
Our results show that the dispersion of herbivore damage from young leaves to older leaves netted the defended plants a fitness benefit only under high levels of herbivore damage (Figure 4.3 \& 4.4). An earlier study addressing an isolated plant-herbivore interaction rather than the aggregated effect of an entire herbivore community found that damage to young leaves was more detrimental to plant fitness than damage to old leaves (de Vries et al., 2018b). This earlier study simulated severe herbivore damage, as the herbivore damage was concentrated in a small period of time rather than spread over the entire development of the plant. This shows that the isolated effect of a single plant-herbivore interaction at a given point during the plant's development can be very different from the aggregated effect of an average herbivore community over the entirety of the season. In the context of temporally dispersed rather than temporally concentrated herbivore damage, our results show that the adaptive value of plant defence in a competitive environment relies on reducing herbivore damage rather than dispersing herbivore damage (Figure 4.4). Generalist herbivores are highly susceptible to plant defence and are, therefore, severely hampered in their growth and survival by taxon-specific secondary metabolites (Gols et al., 2008; Poelman et al., 2008a). However, most specialist herbivores are mildly hampered by the plant's defence (Poelman et al., 2008a; Wei et al., 2015), despite feeding from well defended yet highly nutritional young leaves (Feeny, 1976; Cates, 1980). This leads us to predict that defence is disfavoured when plants are under attack by specialist herbivores as the benefits of defending against a specialist herbivore are less likely to outweigh the costs of the defence investment. This prediction supports data by Lankau (2007), who showed that generalist and specialist herbivores exert opposing selection pressures on plant defence, where high levels of defence are favoured in the absence of specialist herbivores and disfavoured in the absence of generalist herbivores. The negative selection pressure of specialist herbivores is further strengthened by the role of secondary metabolites in food-plant selection by specialists, making plants with a high level of defence more vulnerable to attack by specialist herbivores (Poelman et al., 2008a; Badenes-Pérez et al., 2010; Poelman et al., 2010; BadenesPerez et al., 2014). The study of invasive plant species might shed light on the selective pressure exerted by generalist and specialist herbivores on plant defence as invasive plant species experience herbivore communities that often lack their native specialist herbivores. These invasive plant species show increased competitive ability, are more resistant to generalist herbivores but are less resistant to specialist herbivores compared to their native conspecifics (Lin et al., 2015). This might indicate that not only the level but also the complexity of plant defence is under different selection by herbivore species with different levels of specialisation 
(Lankau \& Strauss, 2008). A more complex blend of secondary metabolites is costlier for the plant to produce, while the benefits are dependent on the attacking herbivore. As a result, the complexity in secondary metabolites potentially plays an important role in determining the adaptive value of plant defence.

Plants growing in high densities maximise their ability to compete for light through a suite of morphological changes such as increased internode elongation and leaf hyponasty, termed the shade avoidance syndrome (Fraser et al., 2016; Ballaré \& Pierik, 2017). These morphological changes are regulated by the ratio of red to far-red (R:FR) light in the spectrum reflected within a canopy, which is a robust signal of neighbour presence as plant tissues readily absorb red light while the far-red light is reflected or transmitted (Ballaré et al., 1990). This low R:FR signal also reduces the plant's defensive capabilities by desensitising the plant to jasmonic acid (JA), one of the essential phytohormones that regulate plant defence (Moreno et al., 2009; de Wit et al., 2013; Ballaré, 2014; Campos et al., 2016). The mediation of defences by R: FR is indicative of an interactive effect on selection pressure between herbivory and competition and multiple functions of this mechanism to regulate the plant phenotype have been identified (de Vries et al., 2017). The most obvious function of this mechanism is a resource-driven tradeoff between the ability of a plant to defend against attackers and its ability to outcompete its neighbours (Herms \& Mattson, 1992; Ballaré, 2014), as is apparent when comparing strategies of different plant species. Alternatively, this mechanism could be a means of optimal defence partitioning within the canopy (McKey, 1974; Izaguirre et al., 2013), increasing defence investment towards leaves that represent the highest investment in current and future resource acquisition. This gradient in defence partitioning could function to disperse herbivores within the canopy, driving generalist herbivores away from younger and more valuable leaves. A third possibility is a mechanism to reduce plant defence expression as a whole, to decrease plant attractiveness to specialist herbivores (Poelman \& Kessler, 2016), which are potentially more harmful to plant competitiveness than generalist herbivores (de Vries et al., 2018b). Each of these non-exclusive hypotheses is a potential explanation for and may have contributed to the downregulation of defences by R:FR. Our results have shown that inter-genotypic competition for light is a highly impactful driver of plant fitness that potentially plays a strong role in determining the adaptive value of plant defence. Our results also suggest that the most likely function of the downregulation of defences by R:FR lies in reducing overall costs of defence while optimising the benefits provided, rather than shaping the distribution of herbivores within the plant. However, our model does not regard single plant-herbivore interactions, which are 
more variable in space and time and therefore might have a much stronger impact on the adaptive value of plant defence than suggested by the results of this study (Poelman \& Kessler, 2016; de Vries et al., 2018b).

In this study, we focussed on one possible ecological interaction in a single ecological setting to highlight the importance of studying these interactions to understand the way plants function. We focussed on Brassica nigra as a model plant, which warrants the chosen set of conditions as it often occurs in dense mono-stands. However, the growing conditions faced by other plant species are likely very different from those of Brassica nigra. We focussed on a single form of competition, for light, while plants compete for a plethora of other critical resources such as water, nitrogen and phosphorous. Knowledge on the physiology and ecology of competition for light is well established (Ballaré \& Pierik, 2017) and great progress is being made on the physiology of root architectural responses to nutrient availability (Bisseling \& Scheres, 2014) and their effects on nutrient competition (Rasmussen et al., 2017). This study focusses on a generic plant-herbivore-interaction while we know from studies on plantherbivore communities (Poelman \& Kessler, 2016) and the rhizosphere microbiome (Berendsen et al., 2012; Philippot et al., 2013; Mommer et al., 2016) that the individual interactions in these complex communities can be highly species-specific yet play a major role in plant performance (Berendsen et al., 2018).

A next step in elucidating how the plant balances growth and defence is to place more emphasis on the temporal aspects of plant-herbivore interactions. The expression of defences on the plant level changes during the development of the plant and is more variable than can be expected based on the ontogenetic defence trajectory at the leaf level (Barton \& Boege, 2017). The costs and benefits of plant defence as well as the impact of herbivore damage are all relative to the plant's developmental stage (Boege \& Marquis, 2005). Herbivore infestation early in development is potentially far more devastating to plant fitness as well as the plant's ability to outcompete neighbours than an infestation in later stages of development. Additionally, a herbivore can move to a neighbouring plant during the most voracious stage in its development to avoid both the induced defences and reduced feeding potential of its host plant (van Dam \& Baldwin, 1998), a dynamic that is not included in the scope of this paper. When assessing the costs and benefits of defences on plant competitiveness, we should consider that the costs are paid at an earlier moment during plant development than the benefits are reaped. The time between these events and the predictability of this time interval are also potentially important 
drivers of selection towards induced or constitutive defences. These temporal interactions are another potential source of ecological costs in addition to competition for light and infestation with generalist or specialist herbivores, which highlights how the costs and benefits of plant defence are primarily dependent on ecological interactions.

\section{Acknowledgements}

This work was supported by the Netherlands Organization for Scientific Research (NWO) (project number: 823.01.004) and the European Research Council (ERC) under the European Union`s Horizon 2020 research and innovation programme (grant agreement nr: 677139 to EHP). We thank Niels Anten and the reviewers for comments on an earlier version of the manuscript.

\section{Supporting Information}

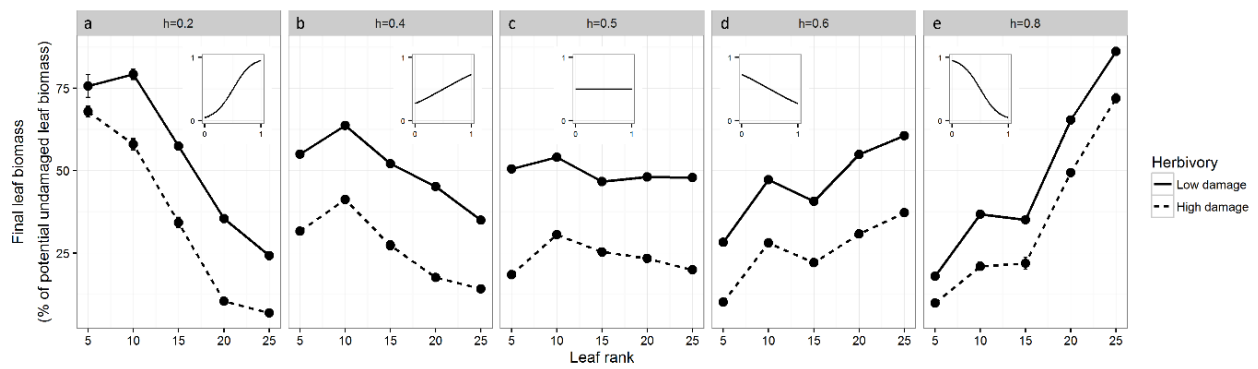

Figure S4.1. The final leaf biomass as a percentage of potential undamaged leaf biomass (yaxis) of different leaf ranks (x-axis, $5=$ old leaves at the bottom of the canopy, $25=$ young leaves at the top of the canopy) for different levels of herbivore damage (low, high) and different herbivore distribution patterns (panels, $\mathrm{h}$ in eq. 4.3 ) at a $15 \%$ plant defence investment. The subplots show the herbivore distribution (y-axis) as a function of relative leaf rank (x-axis, lowest leaf rank $=0$, highest leaf rank $=1$ ) for the corresponding value of $h$. Error bars show standard error of the mean. 



\title{
Chapter 5
}

\section{The ecological significance of light quality in optimising plant defence}

\author{
Jorad de Vries ${ }^{1,2 \S}$, Jacob C. Douma ${ }^{1,2, * \S, ~ E r i k ~ H . ~ P o e l m a n ~}{ }^{1}$, Marcel Dicke ${ }^{1}$, \\ Niels P.R. Anten², Jochem B. Evers ${ }^{2}$ \\ ${ }^{1}$ Wageningen University, Laboratory of Entomology, Wageningen, The Netherlands \\ ${ }^{2}$ Wageningen University, Centre for Crop System Analysis, Wageningen, The Netherlands \\ $\S \mathrm{JdV}$ and JCD should be considered joint first author
}




\title{
Chapter 5
}

\begin{abstract}
Plants balance the allocation of resources between growth and defence to optimise fitness in a competitive environment. Perception of neighbour detection cues, such as a low ratio of red to far-red radiation (R:FR), activates a suite of shade-avoidance responses that include stem elongation and upward leaf movement, while simultaneously downregulating defence. This downregulation is hypothesized to benefit the plant either by mediating the growth-defence balance in favour of growth in high plant densities, or, alternatively, by mediating defence of individual leaves such that those most photosynthetically productive are best protected. To test these hypotheses, we used a 3D functionalstructural plant model of Brassica nigra that mechanistically simulates the interactions between plant architecture, herbivory and the light environment. Our results show that plant-level defence expression is a strong determinant of plant fitness and that leaf-level defence mediation by R:FR can provide a fitness benefit in high densities. However, optimal plant-level defence expression does not decrease monotonically with plant density, indicating that R:FR mediation of defence alone is not enough to optimise defence between densities. Therefore, assessing the ecological significance of R:FR mediated defence is paramount to better understand the evolution of this physiological linkage and its implications for crop breeding.
\end{abstract}




\section{Introduction}

Plants growing in high densities suffer more from pests and diseases compared to plants in low densities (Burdon \& Chilvers, 1982). As many plants in nature and most of our food crops grow at high densities, exploring the causes and functionality of this phenomenon is of both scientific and societal interest. A number of explanations have been proposed for this phenomenon, such as the relation between plant density and light availability, microclimate and dispersal ability of pathogens or pests (Ratnadass et al., 2012). Recently, evidence has accumulated that the same density-dependent light cues play an important role in regulating defence levels as well as shade avoidance responses (Ballaré \& Pierik, 2017). To avoid future shading by their neighbours, plants exhibit a suite of responses to bring leaves into a favourable position with respect to the light gradient. These responses are termed the shade avoidance syndrome (SAS) and include, but are not restricted to, stem elongation, an upward change of the leaf angle, and petiole elongation (Fraser et al., 2016; Ballaré \& Pierik, 2017).

An important light cue that is used by plants to detect future shading is the red to farred ratio (R:FR) (Ballaré et al., 1990). Red light is readily absorbed by plant tissues while most far-red radiation is reflected and transmitted. Hence, the R:FR ratio in a developing canopy decreases over time, and R:FR will be lower in dense canopies than in open canopies. In addition to inducing a shade avoidance response, a low R:FR ratio represses the activity of the jasmonate pathway - a phytohormonal pathway involved in plant defence against necrotrophic pathogens and chewing insects (Leone et al., 2014). In this way, shade-avoidance responses and defence are intertwined at the level of signal transduction networks through a common light cue, R:FR, resulting in an increased susceptibility to pathogens and insects in competitive environments characterized by a low R:FR (Moreno et al., 2009; de Wit et al., 2013; Leone et al., 2014).

Two important hypotheses have been put forward why plants downregulate their defence when perceiving a low R:FR (de Vries et al., 2017). First, it is hypothesised that defence is suppressed by a low R:FR ratio to balance growth and defence, such that competitive strength through SAS is favoured over defence at high plant density (Ballaré \& Pierik, 2017). This downregulation may be understood from the perspective of evolutionary game theory (EGT) (Maynard Smith, 1982; Anten \& Vermeulen, 2016). EGT predicts that in a competitive environment natural selection can drive expression of plant traits away from the population level optimum (hereafter called simple optimum) and towards a competitive optimum (Parker 
\& Smith, 1990), which is termed a tragedy of the commons (TOC) (Falster \& Westoby, 2003; McNickle \& Dybzinski, 2013). Competition for resources may lead to overexpression of plant traits related to resource acquisition at the expense of other traits, such as defence against herbivores, leading to trade-offs between growth and defence related traits (Züst \& Agrawal, 2017). Thus, one would expect the optimal level of defence in high plant densities to be lower than the optimal level of defence at lower plant densities, making the R:FR ratio a signal that can potentially regulate plant defence expression between plant densities.

Second, it is hypothesized that R:FR regulates defence expression to optimise the distribution of defences within the plant. The locally perceived R:FR ratio signals the future value of a leaf in terms of photosynthetic gain, warranting the regulation of leaf defence expression by R:FR (Izaguirre et al., 2013; Ballaré, 2014). Plants are known to vary the defence investment of a leaf as a function of the value of that leaf in terms of the current investment of limited resources and the future acquisition of those resources (Koricheva \& Barton, 2012), as predicted by optimal defence theory (McKey, 1974; Cipollini et al., 2014). Differences in leaf value arise from the light gradient in the canopy, with leaves in the bottom of the canopy generally being of lower value due to them intercepting less light and therefore having less potential for photosynthetic gain. Furthermore, leaf defence levels are often correlated to leaf age as plants move secondary metabolites away from older leaves and towards younger leaves that are generally situated in areas of high light intensity (van Dam et al., 1995; Lambdon et al., 2003), but see (Zangerl, 1986).

The downregulation of defences by a low R:FR ratio can have negative consequences for crops if this response makes their defence levels fall below the simple optimum in high plant densities, in which crops are typically grown (Campos et al., 2016). This suboptimal defence expression at the population level can potentially be the result of a TOC driven by an internal resource trade-off between defence and shade avoidance growth. To counteract this potential for suboptimal defence expression in crop plants, it has been suggested to decouple defence from neighbour proximity perception in crops so that their defence expression is not lowered in high planting densities (Campos et al., 2016; Ballaré \& Pierik, 2017). This decoupling potentially increases crop performance by elevating plant defence expression towards the simple optimum, and can reduce pesticide use by elevating the plant's natural ability to defend itself. However, decoupling defence from R:FR signalling potentially impairs the ability of the plant to distribute defences within the canopy according to leaf value. Optimising defence 
distribution in the canopy can increase plant performance by maximising defence expression in the most valuable leaves while reducing costs by minimising defence expression in less valuable leaves.

The aim of this study is to elucidate the role of R:FR in mediating defence expression across densities and within a canopy to optimise plant fitness in an evolutionary setting and how this translates to crop performance. Therefore, we aim to quantify i) the optimum level of whole-plant defence expression under intra-genotypic competition, ii) the optimum level of whole-plant defence expression under inter-genotypic competition, iii) how defence mediation by locally perceived R:FR cues impacts plant fitness under inter-genotypic competition and iv) how decoupling R:FR regulation of defence affects plant fitness in mono-stands.

The interaction between plant competition for light, and its impact on the optimum level of defence expression under herbivore attack is studied using functional-structural plant (FSP) modelling (Vos et al., 2010; Evers, 2016; Evers et al., 2018). FSP models simulate the growth and development of individual plants in three dimensions using source-sink dynamics (Evers et al., 2010; Evers \& Bastiaans, 2016) and functional responses to light cues (Evers et al., 2007; Bongers et al., 2018; de Vries et al., 2018b), as they compete for light. FSP modelling is the only approach that mechanistically simulates the feedback between plant architecture and light cues such as R:FR, that change plant architecture, which in turn modifies the R:FR cue, etc.

\section{Methods}

To elucidate the effect of R:FR regulation of plant defence on plant fitness in a competitive environment, we used Brassica nigra as model species. B. nigra occurs in a wide range of densities and is known to elicit strong architectural responses to changes in R:FR, making it an exellent model plant for this study. We expanded an FSP model of $B$. nigra growth and development (de Vries et al., 2018b), which has been developed in the FSP modelling platform GroImp (Hemmerling et al., 2008). The model mechanistically simulated plant growth and development through light-driven source-sink dynamics and functional responses of plant architecture to R:FR cues (for a detailed model description see de Vries et al. (2018b)). The architectural responses to changes in R:FR that determine stem elongation, branching, leaf size and leaf angle were parameterised and tested using detailed field measurements on architecture, biomass and seed production (de Vries et al., 2018b). Plant performance and fitness were emergent properties of the model that arose from dynamic interactions between plants and the feedback between plant architecure and the light environment, as altered by plant growth and 
leaf herbivory.

We expanded the existing model with a plant defence module that described defence expression at the leaf level as a function of local R:FR perception. The investment in plant defence interacted with plant growth through source-sink dynamics, and reduced herbivore damage, as explained in the sections below.

\section{Plant defence}

The model described plant defence as the biomass allocated towards a pool of secondary metabolites that may be distributed over the leaves of the plant. The model distinguished between two groups of plant strategies with either 1) a constant leaf defence expression or 2) a leaf defence expression regulated by local R:FR perception. For the first group, the potential leaf defence expression ( $D_{\text {pot,i, }}$ g defence (e.g. secondary metabolites)) of leaf $i$ was a function of the biomass of the leaf $\left(L_{\mathrm{i}}, \mathrm{g}\right)$ and the maximum leaf defence expression $\left(D_{\max }, \mathrm{g}\right.$ defence $\mathrm{g}^{-}$ ${ }^{1}$ leaf), which is realised if resources are not limiting.

$$
D_{\text {pot }, i}=D_{\max } * L_{i}
$$

For the second group, in which the potential leaf level defence expression ( $D_{\text {pot,i, }}$ g defence) was regulated by the perceived R:FR, we assumed the potential level of defence of that leaf $\left(D_{p o t, i}\right)$ to be related to the R:FR perceived at the leaf tip (eq. 5.2)(Pantazopoulou et al., 2017). The functional form relating defence expression to R:FR is not known. For this reason, a sigmoidal relationship was chosen to ensure that $D_{p o t, i}$ does not reach levels below a minimum or above a maximum level of defence at low and high R:FR ratios respectively. Furthermore, we tested a range of parameter combinations of the logistic functions to simulate different defence responses to R:FR (see scenarios below). The potential level of defence was dependent on the maximum leaf defence expression at high R:FR ratios $\left(D_{\max }, \mathrm{g}\right.$ defence $\mathrm{g}^{-1}$ leaf $)$, the biomass of the leaf $\left(L_{\mathrm{i}}, \mathrm{g}\right)$, the R:FR perception on the leaf tip $\left(\mathrm{R}: \mathrm{FR}_{\mathrm{i}}\right)$ and a parameter that describes the R:FR ratio where $D_{\text {pot,i is }} 0.5^{*} D_{\max }$ (R:FR 0 ; eq. 5.1, Table 1 for parameter values). $D_{\text {pot,i }}$ reflected the defence level that is realised if resources are not limiting.

$$
D_{p o t, i}=\frac{D_{\max } * L_{i}}{1+e^{-10\left(R: F R_{i}-R: F R_{0}\right)}}
$$

At every time step, the potential biomass a plant allocated to defence was calculated from the difference between current whole plant defence biomass and potential defence expression of all 
leaves on the plant. The energy requirement of maintaining and growing the pool of secondary metabolites available to the plant was called the sink strength (Sink $k_{D}$ g glucose/d). Sink consisted of two components: a cost to construct new defensive compounds and a cost to maintain current defence (eq. 5.3). The construction costs included the machinery to synthesise, transport, re-allocate and store defensive compounds such as glucosinolates, and were calculated by multiplying the requested defence investment with the construction costs of defence ( $c c, \mathrm{~g}$ glucose $\mathrm{g}^{-1}$ defensive compound). The costs of maintenance were calculated from the current whole plant defence biomass $(D, \mathrm{~g})$ and a parameter for maintenance respiration

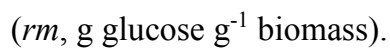

$$
\operatorname{Sink}_{D}=c c\left(\sum_{i=1}^{n} D_{p o t, i}-D\right)+r m D
$$

With $D_{\text {pot,i }}$ representing the potential defence investment in an individual leaf $i$, and $r m$ the maintenance costs of the current pool of secondary metabolites. Note that the suppression of defence by R:FR acted through $D_{\text {pot,i }}$ and hence lowered the defence sink strength.

The amount of substrate that could be allocated to defence $\left(C_{\mathrm{D}}, \mathrm{g}\right)$, depended on the available assimilates $(S, \mathrm{~g})$ and the combined sink strength of all sink organs in the plant (Sink, g). We assumed that, when assimilates were limiting, all plant organs received assimilates proportional to their demand (eq. 5.3) (Evers et al., 2010; Evers \& Bastiaans, 2016).

$$
C_{D}=\min \left(\operatorname{Sink}_{D}, \frac{S * \operatorname{Sink}_{D}}{\sum_{i=1}^{n} \operatorname{Sink}_{, i}}\right)
$$

The pool of secondary metabolites available for distribution in the next time step $\left(D_{\mathrm{t}+1}, \mathrm{~g}\right)$ was calculated from the pool of secondary metabolites of the current time step $(D, \mathrm{~g})$, adding the assimilates allocated to defence while subtracting maintenance and construction costs.

$$
D_{t+1}=D+\frac{C_{D}-D * r m}{c c}
$$

The pool of secondary metabolites was then distributed over the leaves, with the defence allocated to a leaf $\left(D_{\mathrm{i}}, \mathrm{g}\right)$ being proportionate to their contribution to the total potential plant defence expression $\left(\Sigma D_{\text {pot,i, }}, \mathrm{g}\right)$.

$$
D_{i}=\frac{D_{p o t, i} D_{t+1}}{\sum_{i=1}^{n} D_{p o t, i}}
$$




\section{Chapter 5}

\section{Herbivore damage}

Herbivore damage was simulated by assuming that potential leaf damage was proportional to the biomass of the leaf, irrespective of its position in the canopy. This baseline assumption was made to ensure that there was no a priori advantage of distributing leaf defence to a given position in the canopy or leaves of a given size. The actual leaf damage depended on the defence level of that leaf and the feeding rate of the insect herbivore. We assumed that feeding rate increased linearly with thermal time to match plant growth and development (tt GDD (growing degree days)) (Bale et al., 2002).

$$
h_{i}=h_{\text {pot }}\left(1-\frac{\frac{D_{L, i}}{L_{i}}}{\frac{D_{L, i}}{L_{i}}+c}\right) t t
$$

With herbivore damage on the leaf level ( $\mathrm{h}_{\mathrm{i}}$, dimensionless) being a function of the potential feeding rate per unit of growing degree days $\left(h_{\mathrm{pot}}, \mathrm{GDD}^{-1}\right)$, the thermal time of the current time step ( $t t, \mathrm{GDD})$, the defence present in the leaf $\left(D_{\mathrm{i}}, \mathrm{g}\right)$ relative to the biomass of the leaf $\left(L_{\mathrm{i}}, \mathrm{g}\right)$ and a parameter $c$ ( $\mathrm{g}$ defence $\mathrm{g}^{-1}$ leaf) that described the leaf defence level at which herbivore damage was reduced by half.

The proportion herbivore damage to a leaf $\left(h_{i}\right)$ reduced leaf biomass $\left(L_{\mathrm{i}}\right.$, g, eq. 5.7). We assumed that specific leaf area was constant over time and over leaf ranks and that defensive compounds were homogeneously distributed within a leaf, so $h$ was linearly related to a reduction in leaf area and leaf defence.

$$
\frac{d L_{i}}{d t}=-L_{i} h_{i}
$$

Table 5.1. Model parameters related for herbivory and plant defence.

\begin{tabular}{lllll}
\hline Description & Name & Value & Unit & eq. \\
\hline Maximum defence investment & Dmax & Variable $(0-0.2)$ & g defence g $^{-1}$ leaf & $5.1,5.2$ \\
RFR where $\mathrm{D}_{\text {pot }}=0.5^{*}$ Dmax & $R: F R 0$ & Variable $(0.45-0.85)$ & umol red umol $^{-1}$ farred & 5.2 \\
Construction costs of defence & $c c$ & 15 & g glucose g ${ }^{-1}$ biomass & $5.3,5.5$ \\
Maintenance respiration rate & $r m$ & 0.02 & g glucose g ${ }^{-1}$ biomass & $5.3,5.5$ \\
Potential herbivore damage & $h$ pot & 0.005 & dimensionless & 5.7 \\
Herbivore damage reduction coefficient & $c$ & 0.18 & g defence g $^{-1}$ leaf & 5.7 \\
\hline
\end{tabular}


1. What is the simple optimum of plants with defence homogeneously distributed over the leafs?

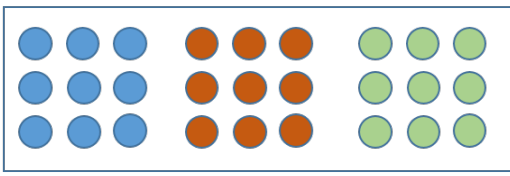

4. What is the seed production of a plants that regulate defence according to R:Fr when grown in mono-stands and how does it compare to the competitive optimum in step 2.

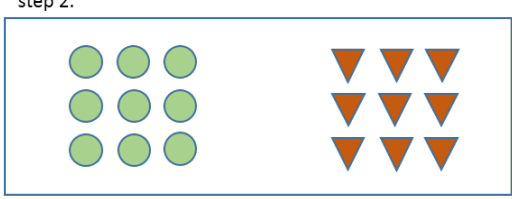

2. What is the competitive optimum of plants with defence homogeneously distributed over the leafs

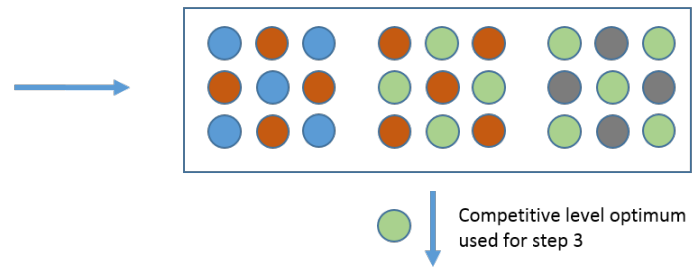

3. What is the competitive optimum of plants that regulate defence according to R:Fr when competing against plants with defence homogeneously distributed over the leafs?

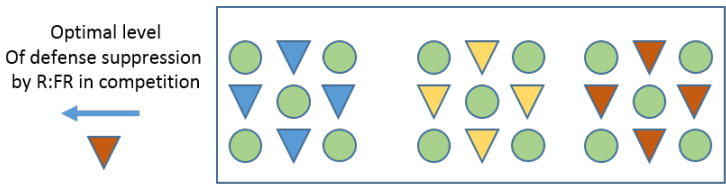

Legend

Homogenous defence across leafs

Leaf defence regulated by local R:FR

Figure 5.1 Schematic outline of the four model scenarios. In the first scenario (top left), we seek for the simple optimal level of defence of plants that homogeneously defend their leaves. In the second step, we test the competitive optimum level of defence, by competition between individuals that homogeneously distribute defence over the leaves that vary slightly in their level of defence. Competing strategies are positioned in a checkerboard design. The competitive optimum is selected to compete with plants that regulate defence by R:FR (scenario 3). Plants that regulate defence by R:FR and perform better compared to plants that homogeneously defend their leaves are grown in mono-stands to test if the benefit of regulating defence still pays-off when growing in competition with plants that have the same strategy (scenario 4). Each scenario is repeated in three densities to explore whether a plant that regulates defence by R:FR can approach the competitive optimum in each density through a single response curve. See main text for definitions of simple and competitive optimum.

\section{Simulations}

To test whether regulation of defence by R:FR is beneficial to plant fitness we compared the performance of plants that had a constant defence distribution over their leaves (group 1) with plants that regulated the leaf defence expression by local R:FR perception (group 2). We tested a variety of plant defence strategies that differed in the maximum level of defence expression (Dmax in eq 1 and 2. Ranging from 0, entailing no defence expression, to 0.2) and the distribution of defence over their leaves, which is either constant (group 1) or regulated by R:FR (group 2; R:FR ${ }_{0}$ in eq 2. ranging from 0.45 to 0.85 ). Seed production of a strategy after one growing season was taken as measure of its fitness, which was reasonable considering that we model an annual plant. The model simulated plots of four by four plants, where plants competed either with neighbours adopting the same strategy (mono-stands; intra-genotypic competition) 


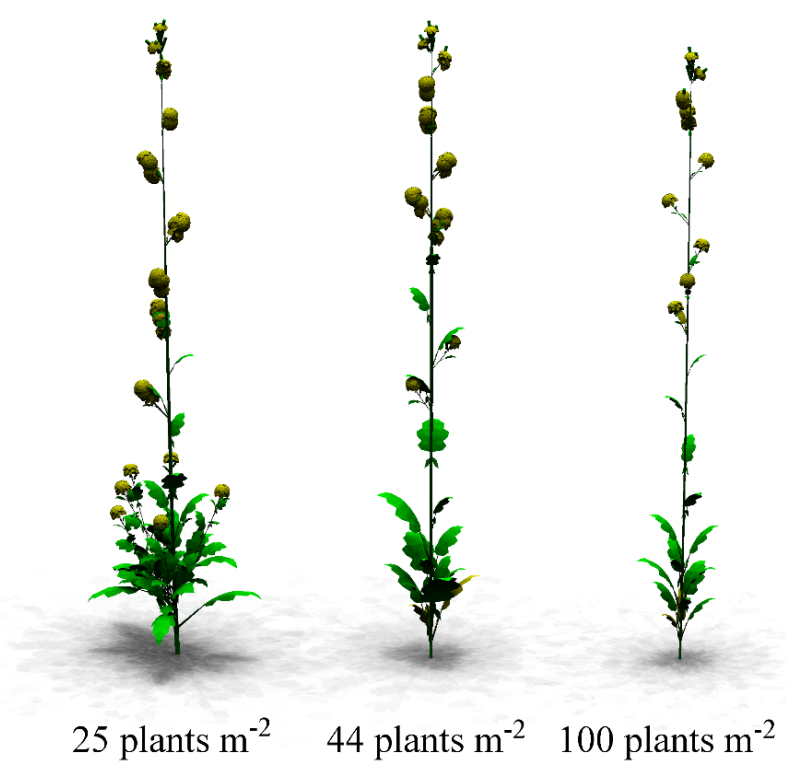

Figure 5.2 Visual representation of the B. nigra phenotype in three plant densities, showing the architectural changes related to branching dynamics, leaf size and leaf angle in response to plant density.

or neighbours adopting a different strategy with the two plant strategies arranged in a checkerboard pattern (mixed stands; inter-genotypic competition). This plot was cloned 625 times to simulate a large field of 10.000 plants for light model calculations. Every time step, the model calculated the light interception of an individual plant by averaging the light interception of its 625 clones. This approach was used to eliminate border effects in light conditions and their effect on plant growth. The simulations spanned a growing season of 124 days (31 March to 2 August), with average daily temperature, average daily insolation and solar angle typical for the Netherlands at a latitude of $52^{\circ}$.

\section{Scenarios}

We simulated four subsequent scenarios to elucidate the effect of defence regulation by R:FR in the context of natural selection and crop performance in a range of plant densities $(100,44$ and 25 plants $/ \mathrm{m}^{2}$ ) (see Figure 5.1 for an overview).

1. First, we determined the optimal level of defence for plants with a constant defence distribution over their leaves (group 1), growing in one of three plant densities $(25,44$ 
Table 5.2 Optimal level of defence at various plant densities and the respective seed output

\begin{tabular}{lllll}
\hline $\begin{array}{l}\text { Plant density } \\
\left(\text { plants } / \mathrm{m}^{2}\right)\end{array}$ & $\begin{array}{l}\text { Simple } \\
\text { optimum } \\
\text { defence }(\mathrm{g} / \mathrm{g})\end{array}$ & $\begin{array}{l}\text { Competitive } \\
\text { optimum } \\
\text { defence }(\mathrm{g} / \mathrm{g})\end{array}$ & $\begin{array}{l}\text { Seed yield } \\
(\text { simple } \\
\text { optimum) }\end{array}$ & $\begin{array}{l}\text { Seed yield } \\
\text { (competitive } \\
\text { optimum) }\end{array}$ \\
\hline 25 & 0.08 & 0.04 & 2.63 & 2.23 \\
44 & 0.12 & 0.06 & 1.49 & 1.28 \\
100 & 0.04 & 0.04 & 1.19 & 1.19 \\
\hline
\end{tabular}

and 100 plants $/ \mathrm{m}^{2}$ ) and competing with plants with the same level of defence. By varying the level of defence expression from $0.0-0.2 \mathrm{~g} / \mathrm{g}$ leaf $\left(D_{\max }\right.$, eq 1$)$ in all plants simultaneously, the level of defence that led to the highest seed production was assumed to be the population level optimum for that density (the simple optimum).

2. Second, we determined the optimal level of defence of plants with a constant distribution of defence over their leaves (group 1) growing in mixed stands in one of three plant densities $\left(25,44\right.$ and 100 plants $\left./ \mathrm{m}^{2}\right)$. We simulated seven strategies, each with an incremental increase in potential leaf defence expression (0-0.12 g/g leaf, with increments of 0.02), and simulated each strategy competing with a less defended strategy and with a better defended strategy (e.g. 0 vs $0.02,0.02$ vs $0.04,0.04$ vs 0.06 etc.). The strategy that outcompeted both the less defended and the better defended competitor was assumed to express the optimal level of defence under inter-genotypic competition at the given plant density (the competitive optimum).

3. Third, we tested the possible fitness benefit of regulating defence by R:FR for plants growing in mixed stands in one of three plant densities $\left(25,44\right.$ and 100 plants $\left./ \mathrm{m}^{2}\right)$. In each stand plants were arranged in a checkerboard pattern where plants that regulated defence by R:FR (group 2) were competing with plants with a constant leaf defence expression (group 1) that matched the competitive optimum obtained from scenario 2. We tested 20 strategies of R:FR regulating plants that differed in their maximum leaf defence expression ( $D_{\max }$, eq 2, 0.4-1.2 in steps of 0.2) and their sensitivity to the R:FR signal $\left(R: F R_{0}\right.$, eq 2, 0.45-0.85 in steps of 0.1$)$. By varying both parameters we explored the benefit of R:FR regulation as a way to distribute defence according to expected leaf 


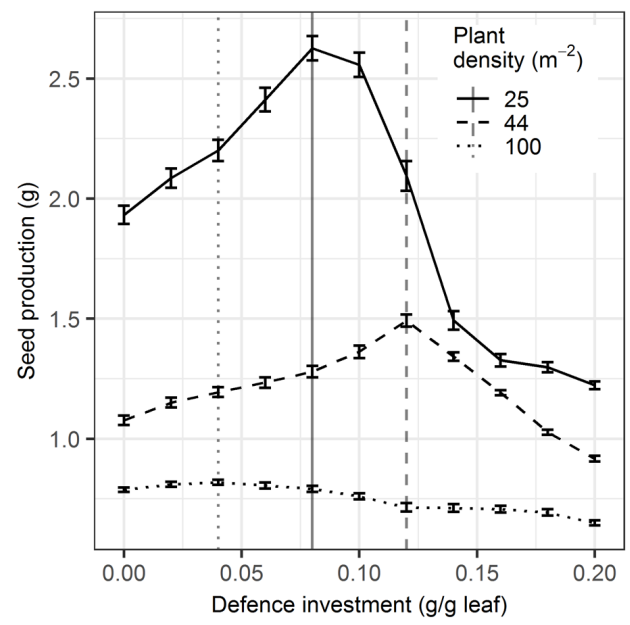

Figure 5.3 The relationship between investment in defence (g defence /g plant biomass) and seed production (grams) of plants that distribute defence homogeneously over leaves for three different densities (line type) and a given level of herbivory. The vertical lines denote the optimum levels of defence for each density. Error bars represent standard error of the mean seed output $(\mathrm{n}=20)$.

value and how this distribution of defence impacts the optimal level of whole-plant defence expression.

4. Fourth, to test the consequences of decoupling defence from the R:FR cue when plants are grown in monoculture, we selected the strategies of R:FR regulating plants that outcompeted the plants expressing the competitive optimum with a constant leaf defence expression. These plant strategies were grown in mono-stands and compared to the mono-stand performance of the competitive optimum established in scenario 2 to assess the effect of R:FR mediation of leaf level defence on crop performance.

To account for model stochasticity (caused by random variation in seed orientation, potential leaf and stem length, the maximum number of phytomers) we ran 20 simulation for scenario's 1, 2 and 4, and 40 replications for scenario 3.

\section{Results}

\section{Plant density}

Plant morphology changed with an increase in plant density, which reduced the number of branches and leaf size, and increased leaf angle (Figure 5.2). In addition, the R:FR ratio perceived by the plants differed across densities, both temporally and spatially (de Vries et al., 

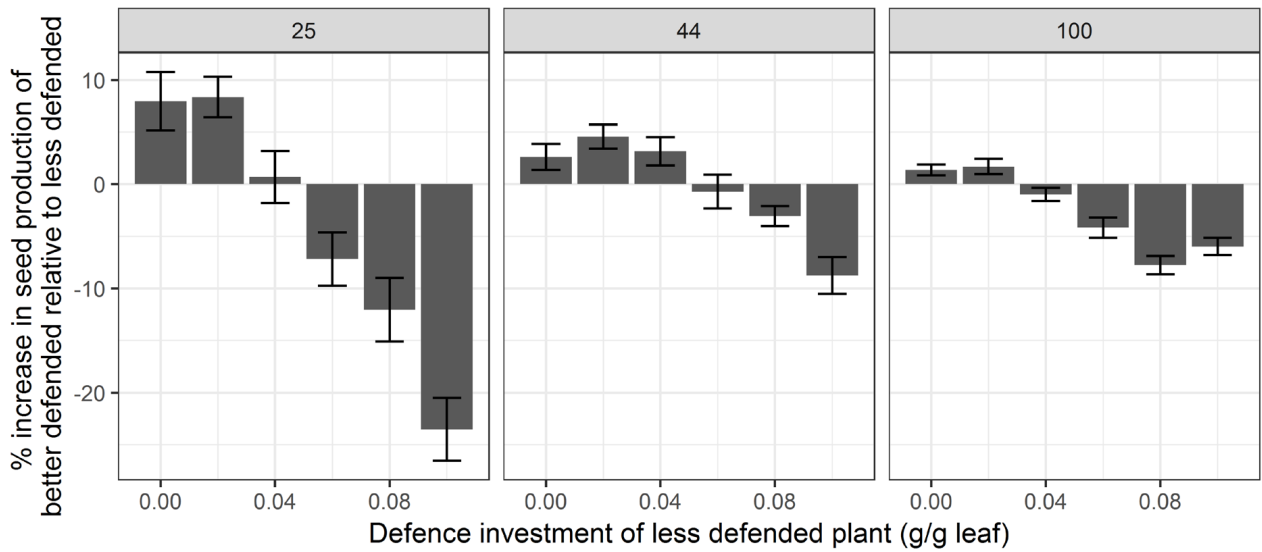

Figure 5.4 The relative change (\%) in seed production for plants that invest $0.02 \mathrm{~g} / \mathrm{g}$ more in defence compared to the defence investment $(\mathrm{g} / \mathrm{g})$ of the lesser defended competitors in three plant densities $\left(25,44\right.$ and 100 plants $\left./ \mathrm{m}^{2}\right)$. At low level of defence, it pays off to be better defended (increase in fitness). Increasing defence investment above an intermediate level of defence investment does not pay off anymore. This defence level is defined as the competitive optimum. Error bars represent standard error of the mean seed output $(n=20)$.

2018b). The initial drop in R:FR was stronger and came earlier with an increase in plant density, initiating the morphological changes associated to the increase in plant density (Figure S5.3).

\section{Scenario 1}

In the first scenario, the optimal defence level of plants that distribute defence homogeneously over their leaves was determined (simple optimum; Figure 5.3). At all densities, an optimum defence level was found that yielded highest seed production, represented by the peaks in the lines presented in Figure 5.3. The optimal level of defence was highest at 44 plants $/ \mathrm{m}^{2}$ at 0.12 gram defence per gram leaf. At the low density of 25 plants $/ \mathrm{m}^{2}$ and the high density of 100 plants $/ \mathrm{m}^{2}$, the optimum defence levels were lower: 0.08 and 0.04 gram defence per gram leaf, respectively.

\section{Scenario 2}

In the second scenario, the competitive optimum level of defence expression was determined by simulating mixtures of two plant strategies, one better defended than the other, with incremental increases in plant defence investment in both strategies. At lower defence investments, the better defended plants reached a higher fitness than the less defended plants, while at higher defence investments the less defended plants reached a higher fitness than the 


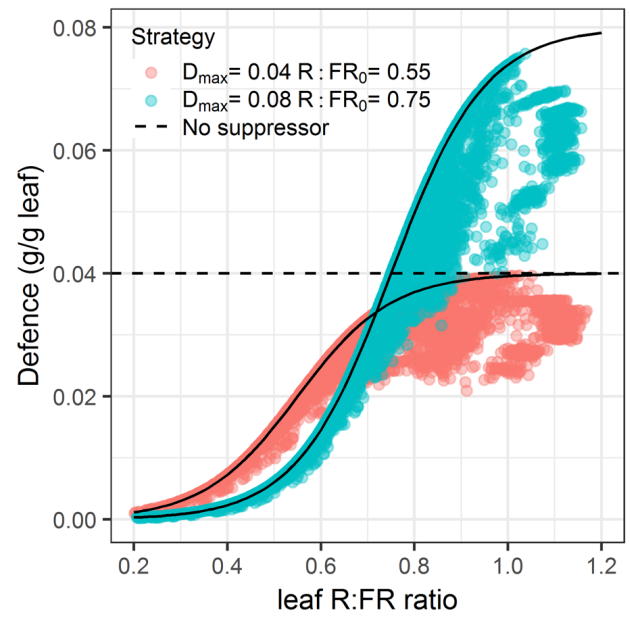

Figure 5.5 The relationship between R:FR ratio perceived by the leaf and the defence level (g/g leaf) the next day of two representative (out of 20 simulated) strategies that regulate defence by R:FR, one strategy that reduces the overall investment in defence through suppressing defence in leaves with low R:FR (red; $D_{\max } 0.04, \mathrm{R}: \mathrm{FR}_{0} 0.55$ ) and a strategy that has the same overall investment in defence as the strategy that does not regulate defence upon $\mathrm{R}: \mathrm{FR}$, but allocates defence according to the leaf R:FR (blue; $\mathrm{D}_{\max } 0.08, \mathrm{R}: \mathrm{FR}_{0} 0.75$ ). The black lines represent the potential level of defence in the leaf $\left(D_{\text {pot }}\right)$ and the coloured dots the realised level of defence in a leaf $\left(\mathrm{D}_{\mathrm{i}}\right)$. The dotted line represents the level of defence in a plant that distributes defence homogeneously over the leaves. See equation 2 for details.

better defended plants. (Figure 5.4). The transition point, defined as the competitive optimum, was found to depend on plant density and was estimated at 0.04 for a density of 25 and 100 plants $/ \mathrm{m}^{2}$ and 0.06 for plants in a density of 44 plants $/ \mathrm{m}^{2}$. Thus, the competitive optimum level of defence was lower than the simple optimum in two lower densities, while for the highest density the competitive optimum level of defence was equal to the simple defence optimum (Table 2).

\section{Scenario 3}

In plants that regulate their defence expression by R:FR, the potential defence investment at the leaf level was constrained by local R:FR perception of the leaf as described by eq. 5.2, while the actual defence investment was also dependent on the availability of substrates (see Figure 5.5). Moreover, leaf defence levels decreased with leaf rank, except for the youngest five leaves that are still clustered in buds and, therefore, perceive a lower R:FR than expected based on rank (Figure S5.4).

Regulating defence by R:FR was found to be slightly beneficial compared to plants that 

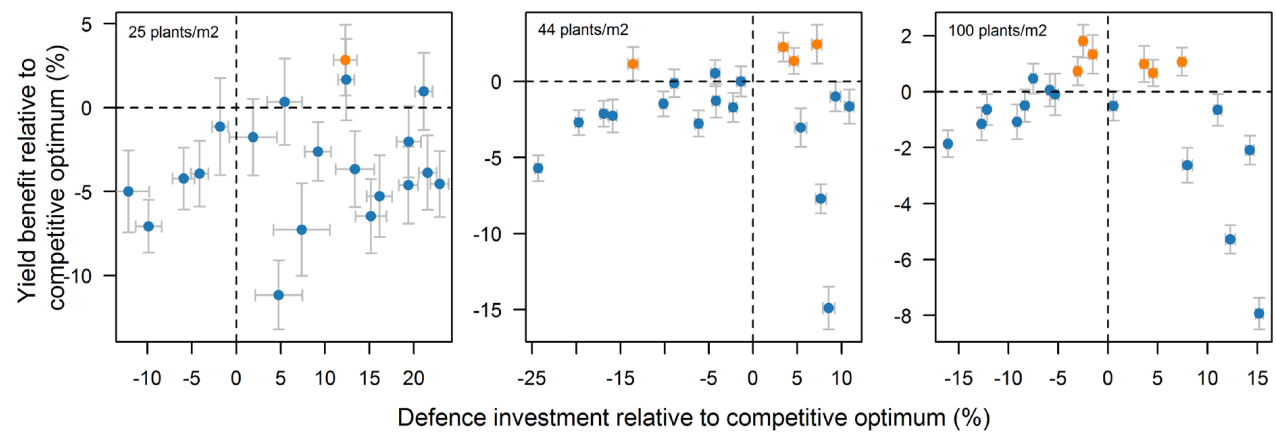

Figure 5.6 Relationship between the mean relative change in investment in defence (\%) and associated change in fitness (\%) of a strategy that regulated defence by R:FR relative to a strategy that distributes defence homogeneously over the canopy. The dots represent different combinations of $\mathrm{D}_{\max }$ (defence investment in leaves with high $\mathrm{R}: \mathrm{FR}$ ) and $\mathrm{R}: \mathrm{FR}_{0}$ representing the R:FR ratio where $D_{\max }$ is $0.5 * D_{\max }$. The orange dots represent strategies that have a fitness benefit and the blue dots strategies that perform equal or worse than a strategy that distributes defence homogeneously over the canopy. Error bars represent standard error of the means and are based on 40 replications).

distribute defence homogeneously over the leaves, but only for specific shapes of the R:FR response curve (eq. 5.2; Figure 5.6; Figure S5.1). Supressing defence in leaves perceiving a low $\mathrm{R}: F R$ ratio without raising the maximum level of defence did slightly pay off at the highest density ( $\mathrm{D}_{\max }=0.04, \mathrm{R}: \mathrm{FR}_{0}=0.45$ in Figure S5.1), but not at the lower densities. In contrast, the $\mathrm{R}: F R$ regulating strategies that outperformed the homogeneously defending plant increased defence investment in leaves perceiving a high R:FR ratio and decreased defence investment in leaves perceiving a low R:FR ratio. For example, at a density of 100 plants $/ \mathrm{m}^{2}$, leaves perceiving a high R:FR ratio (1.2) were defended a factor 1.5-2 higher, while leaves perceiving a low R:FR ratio (0.4) were defended 8-17 times less compared to a homogeneously defended plant.

The benefit of regulating defence in response to R:FR was different for the three densities. At the lowest density only one strategy ( $D_{\max } 0.06$ and $R: F_{0} 0.55$ in Figure $S 5.1$ ) was found to outperform the homogeneous defender, while at the two higher densities, various R:FR response curves were found to perform better than the homogeneous defender. In those densities, the best performing R:FR regulating strategies expressed a whole-plant defence level that was close to that of the competitive optimum level of defence expression (Figure 5.6; Figure S5.2).

\section{Scenario 4}

The strategy with a $\mathrm{D}_{\max }$ of 0.06 and an $\mathrm{R}: \mathrm{FR}_{0}$ ratio of 0.55 approximated the fitness of the best 
Table 5.3. Seed yield (grams \pm se) of the best performing strategies for three different plant densities. For these densities, the performance of the best homogeneous defender, the overall best R:FR regulating strategy across all densities and the best R:FR regulating strategy in a given density are shown.

\begin{tabular}{llll}
\hline Plant density & $\begin{array}{l}\text { Competitive optimum } \\
\text { (Homogeneous defender) }\end{array}$ & $\begin{array}{l}\text { Overall best R:FR regulator } \\
\text { across all densities }\end{array}$ & $\begin{array}{l}\text { Best R:FR regulator in a } \\
\text { given density }\end{array}$ \\
\hline 25 & $2.20 \pm 0.04$ & $2.24 \pm 0.03$ & $2.24 \pm 0.03$ \\
44 & $1.23 \pm 0.02$ & $1.18 \pm 0.02$ & $1.28 \pm 0.02$ \\
100 & $0.81 \pm 0.01$ & $0.81 \pm 0.02$ & $0.82 \pm 0.01$ \\
\hline
\end{tabular}

performing homogeneous defender in each of the three densities most closely, with an average $0.34 \%$ fitness difference. Furthermore, seed production of the two best performing strategies that regulate defence by R:FR when competing against similar individuals was not substantially higher compared to the optimal strategies that do not regulate defence by R:FR (Table 3 ). This shows that although R:FR-regulated defence gives an advantage when competing with plants that distribute defence homogenously over the plant (Figure 5.6), this benefit disappears when competing against plants that adopt the same R:FR regulating strategy.

\section{Discussion}

The aim of this study was to investigate the fitness benefits of regulating defence by R:FR as a mechanism to optimise either i) whole-plant defence expression over a range of densities or ii) the distribution of defence over the leaves such that those with the highest potential for future photosynthetic gain are better protected. The model simulations showed that plant-level defence investment is a strong determinant of plant fitness, and that the optimum defence level varies between densities. However, the optimal plant defence investment did not monotonically decrease with planting density, but was highest at intermediate densities. This suggests that R:FR mediation of plant defence alone is not sufficient to optimise plant level defence expression between densities as the relation between R:FR and plant density does not coincide with the relation between optimal plant-level defence expression and plant density. Our results further show that adjusting leaf-level defence according to the value predicted by its perceived $\mathrm{R}: F R$ ratio can provide a fitness benefit that is more pronounced at higher plant densities.

\section{The benefit of regulating defence by $R: F R$ across densities}

The model analysis showed that the simple and competitive optimum defence levels increased with plant density but decreased again when plant density further increased (scenarios 1 and 2). 
This non-linear relation between optimal defence expression and plant density is the result of the interplay between the costs and benefits of defence, which are in turn affected by the strength of competition, the light environment and density-determined plant phenotype (Figure 5.2). One might expect the highest level of defence at intermediate densities if the costs of defence increase faster with plant density than the benefits of defence. At low density, plants are able to tolerate herbivore damage through an increased capacity for regrowth (Cipollini et $a l ., 2014$ ) and a lower ratio of self to non-self-shading, i.e. removal of leaf area will likely lead to an increased light capture of the underlying leaves of the same plant (Trumble et al., 1993), enabling greater potenial for compensatory growth (Anten et al., 2003). Thus, at low densities, the optimal strategy may be to tolerate herbivory and minimize investment in defence (Strauss \& Agrawal, 1999; McNickle \& Evans, 2018). Conversely, at high densities the ratio of self to non-self-shading is much lower due to the exponential increase in non-self-shading with an increase in plant density and a decrease in plant size due to competition for limited resources. As a consequence, leaf area loss due to herbivory very likely increases the light interception of neighbouring plants rather than of the other leaves of the same plant. Hence, the benefits of defence will increase with plant density as the plant's ability to tolerate herbivory decreases with plant density. The relative direct costs of defence also increase with density, as the photosynthetic gain per unit leaf area decreases with density. Furthermore, any investment in defence comes at the expense of an investment in leaf area or stem growth and reduces future light capture (Douma et al., 2017). Thus, at low densities, plant defence may not pay off because of a strong ability for re-growth and a large degree of self-shading, while at very high densities defence may not pay off because investment in resource acquisition is more important. Hence, one would expect the highest levels of defence at intermediate densities, which is confirmed by the model simulations.

Our model results only partly agree with the hypothesis that plants regulate defence by R:FR to optimally grow and defend at different plant densities (Ballaré \& Pierik, 2017), as optimal defence expression did not decrease monotonically with plant density. Thus, no single strategy that regulated defence by R:FR realised a fitness equal to or higher than the competitive optimum at each of the three densities (Figure 5.6). However, in natural settings, the selective pressure on the sensitivity of defence to R:FR depends on the range and frequency in which possible densities are encountered by the plant, and the selective pressure exerted by these densities. Brassica nigra naturally grows in disturbed soils where initially it may grow solitary, while its seedlings occur at high densities $\left(>50\right.$ per $\left.\mathrm{m}^{2}\right)$ and mostly encounter intra-specific and 
inter-genotypic competition. However, individuals occurring at later successional stages mostly encounter inter-specific competition as other plant species have entered the plant community. The selection pressure exerted at high plant densities is not only dependent on the competitive pressure resulting from the high density, but also on the plant species present in the community and how these interact with other species in the community.

\section{The benefit of regulating defence within a canopy by $R: F R$}

Plants that mediated leaf level defence through locally perceived R:FR cues distributed defensive compounds such that leaves perceiving a high R:FR ratio are relatively better defended and leaves perceiving a low R:FR are relatively less defended compared to plants with a homogeneous defence distribution. This re-allocation of defence within the canopy resulted in a small (a few percent) but distinct fitness benefit that was more pronounced at higher plant densities. However, almost all strategies (14-19 out of the modelled 20) with R:FR mediation of leaf level defence yielded a lower fitness compared to plants that homogeneously defended the plant, most likely because it led to a suboptimal change in whole plant defence expression (Figure 5.6). Particularly at the two highest densities, the strategies that yielded a fitness benefit expressed a whole-plant defence level that was close to the competitive defence optimum. This suggests that the selection on whole-plant defence expression is an important constraint on the optimal distribution of defence within the canopy. Furthermore, the fitness benefit of distributing defence by local R:FR perception arose only in the case of inter-genotypic competition, while under intra-genotypic competition plants hardly benefitted from distributing leaf level defence in response to R:FR (scenario 4).

The optimal relation of leaf-level defence to R:FR was different for each density (Figure 5.6). First, the information relayed by a gradient in the light climate changes with plant density as the ratio of self to non-self-shading decreases with an increase in plant density. Thus, a given $\mathrm{R}: F R$ ratio implies more neighbour shading at high density compared to the same R:FR ratio at a low density, and to gain a competitive advantage at high density plants require a stronger response to R:FR. In reality, plants often use multiple cues, including volatiles, touching, and other light cues, to detect neighbour presence (Pierik et al., 2013) and have a localised perception for these neighbour detection cues (de Wit et al., 2012; Pantazopoulou et al., 2017), which may allow them to better differentiate between self and non-self-shading. Secondly, as the leaf area index increases with density, the gradient in R:FR is steeper in high densities compared to low densities (Figure S5.3). This results in a stronger gradient in leaf value and, 
therefore, stronger selection on a gradient in leaf defence expression. Finally, the variation in fitness benefit of R:FR mediation of defence increased with a decrease in plant density, resulting from a more heterogeneous light environment at the lowest density (as indicated by larger variation in leaf-level photosynthesis; Figure S5.3). This may cause processes such as branching into local canopy gaps to have a relatively big effect on plant fitness compared to the effects of R:FR mediation of defence.

Plants are known to express a higher level of defence in younger leaves (Koricheva \& Barton, 2012), following gradients in the allocation and acquisition of limited resources conform to optimal defence theory. The model developed in this study did not assume a relationship between leaf age and defence expression, but the simulated relation between defence expression and local R:FR perception did predict a qualitatively similar decrease in defence expression over the life span of a leaf as experimental and modelling studies that relate defence to leaf age (Van Dam et al., 1996; Ohnmeiss \& Baldwin, 2000), but see (Barto \& Cipollini, 2005) (Figure S5.4). In dicots such as B. nigra, a low R:FR ratio is expected to coincide with leaf age as the youngest leaves tend to be produced from apical meristems at the top of the canopy where R:FR ratio is high, as opposed to the situation for many grasses. To date, the effect of leaf age and plant development on leaf sensitivity to R:FR is unknown, but is needed to assess the significance of R:FR in regulating defence within the plant. Research on passion flower (Passiflora edulis) in which different branches were exposed to different light quality cues suggests that defence regulation by $\mathrm{R}: \mathrm{FR}$ is a local response that can exist alongside a leaf age regulation of defence expression (Izaguirre et al., 2013). However, in erect herbaceous species such as $B$. nigra, leaf age and leaf R:FR are correlated and the degree to which R:FR can regulate local defence expression independent of leaf age remains to be tested.

The above example illustrates that to fully appreciate the possible benefit of downregulating defence in response to a low R:FR ratio, the effect of plant ontogeny and physiology on sensitivity to R:FR needs to be taken into account. As the R:FR ratio drops quickly early in the development of canopies, and given the asymmetry of competition for light, investing in shade avoidance in that stage is probably more important than to invest in defence - particularly if attack is uncertain. Moreover, as the largest increase in plant defence is observed in the seedling stage (Barton \& Koricheva, 2010), R:FR-dependent regulation of defence may be particularly useful early on in plant development to optimally adapt to the growing conditions. Furthermore, as leaf area accumulates during the growing season, leading to a lower R:FR ratio, regulation 
of defence by R:FR will lead to reduced defence levels over the growing season which may be beneficial as the return on investment of leaves developed later in the season is lower (Douma et al., 2017).

\section{Consequences of decoupling R:FR and defence for crop growth}

It has been suggested that decoupling defence from R:FR in crops could avoid a level of defence expression that is suboptimal for the field-level performance of the crop (Campos et al., 2016; Ballaré \& Pierik, 2017). Decoupling defence from R:FR perception may be a relatively easy way to increase whole-plant defence levels because the JAZ genes responsible for this coupling have been identified in Arabidopsis. However, the benefit of such decoupling depends on the density at which the crop is planted and the difference between the simple and competitive optima in defence expression at that density. The model showed that plant fitness of the competitive optimum was lower compared to that of the simple optimum, pointing to a tragedy of the commons (Augner et al., 1991). Furthermore, the difference between simple and competitive optima was dependent on plant density. An increase in plant density initially increased the difference between simple and competitive optima, but brought the two closer together at high plant density. Therefore, the benefit of decoupling defence and R:FR in crop plants is likely to depend on the density in which the crop is planted. Furthermore, our results suggest that the changes in defence distribution within the canopy as a result of decoupling defence from R:FR hardy affects field level performance, contrary to our initial hypothesis (scenario 4). Yet, whether the decoupling indeed leads to a notable increase in plant defence in (field) crops depends on the importance of other processes governing leaf defence, such as plant development (Barton \& Koricheva, 2010), other light cues such as UV-B (Qi et al., 2018) and plant volatiles (Kegge \& Pierik, 2010; Karban et al., 2013; Cortes et al., 2016).

\section{Interactions between plant defence expression and the herbivore community.}

The model assumes that herbivore damage done to a plant is solely determined by temperature and leaf biomass and is, therefore, assumed to be independent of the interactions between the plant and herbivore communities. Plant-mediated changes in the composition and distribution of the herbivore community can be strong determinants of plant fitness and can, therefore, exert selection pressure on plant defence expression for three reasons (Stam et al., 2014; Poelman \& Kessler, 2016; de Vries et al., 2017). First, the gradient in leaf defence expression within a canopy as established by R:FR can benefit the plant by dispersing herbivore damage away from young leaves (Cipollini et al., 2014), which is shown to benefit plants in competition with 
conspecifics, but only under high herbivore pressure (de Vries et al., 2018a; de Vries et al., 2018b). If this effect would have been included in this study, it would have resulted in a bigger advantage of R:FR-mediated defence. Second, whole-plant defence expression is known to impact host-selection preference of insect herbivores, attracting specialist herbivores and repelling generalist herbivores (Badenes-Perez et al., 2014). Host selection by herbivores based on defence expression means that the herbivore pressure experienced by a plant is not only dependent on its own defence expression but that of its neighbours (Bustos-Segura et al., 2017). This theoretically results in a tragedy of the commons where defence expression exceeds or does not reach optimal values in to deflect herbivore pressure onto neighbouring plants (McNickle \& Dybzinski, 2013). However, we argue that this depends on the composition of the herbivore community. A herbivore community dominated by generalist herbivores should favour high defence expression while a herbivore community dominated by specialists should favour low defence expression (Lankau, 2007). These opposing selection pressures are also observed in invasive plant communities that no longer encounter their native specialist herbivores, making them lose resistance to these specialist herbivores while strengthening their competitive ability (Lin et al., 2015). How those selective forces interact with R:FR mediated defence in a canopy remains to be seen. Finally, the identity and abundance of insect herbivore species may change with planting density, which may lead to changes in the optimal level of defence and the defence response to R:FR predicted by our model (Rhainds \& English-Loeb, 2003; Otway et al., 2005; Joshi et al., 2008). If plants in high densities experience relatively lower herbivory per unit biomass compared to lower plant densities, the optimal level of defence may increase. Thus, the ecological significance of the role of the R:FR ratio in mediation of plant defence needs to be assessed in the light of the temporal and spatial dynamics and the composition of insect herbivore communities (de Vries et al., 2017). 


\section{Supporting Information}

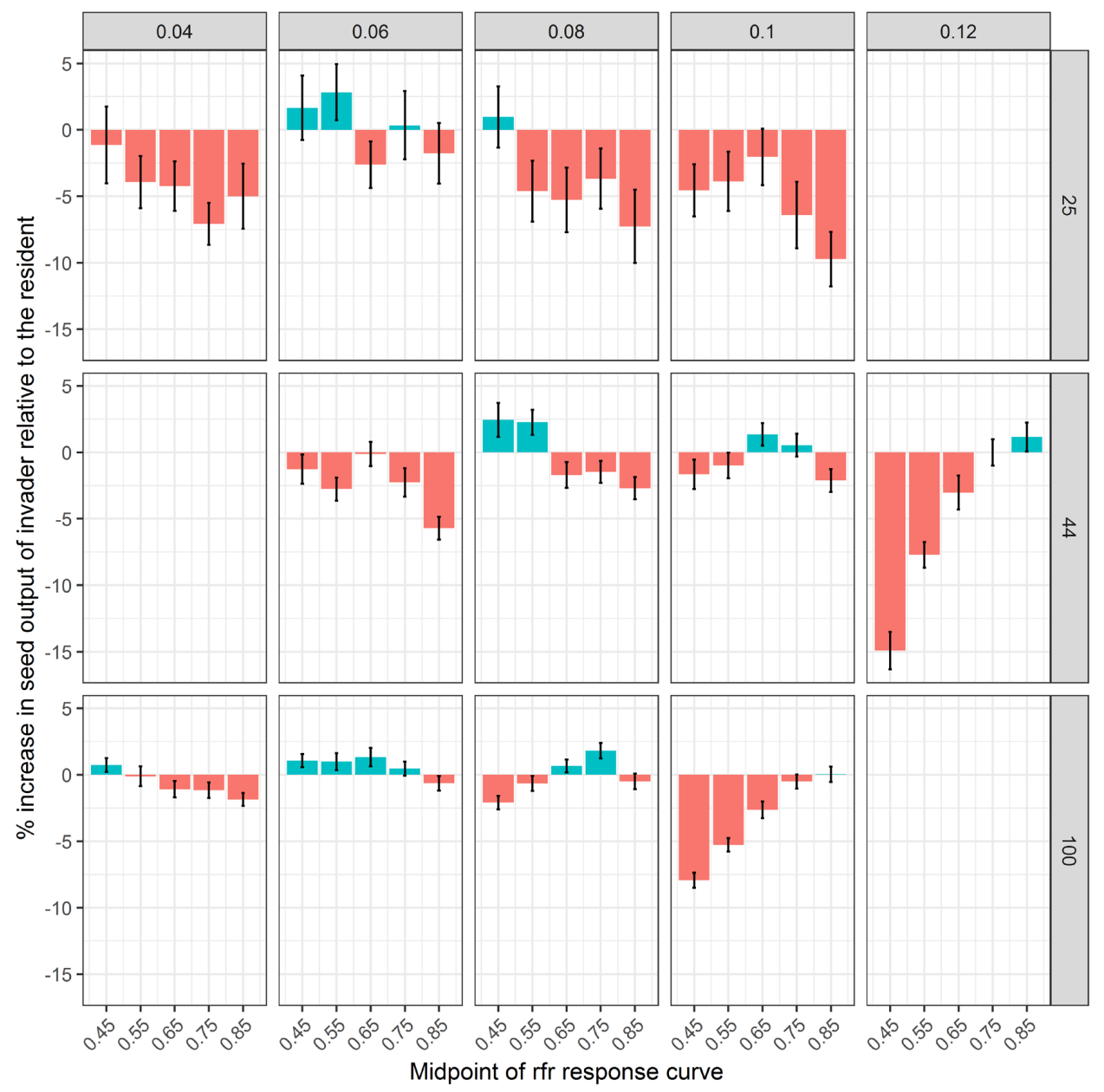

Figure S5.1. The relative increase in seed production (\%) for plants that regulate leaf-level defence by R:FR when competing against plants that distribute defence homogeneously over the canopy (blue: increase in fitness, red decrease in fitness) (scenario 3). Left panel: strategies that compromise total defence investment by suppressing defence in leaves with low R:FR (budget cut). The budget cut increases with increasing midpoint (R:FR $)$ values. Right panels: strategies that reallocate defence by R:FR with varying $\mathrm{D}_{\max }$ (columns). The rows represent three planting densities $\left(100,44\right.$ and 25 plants $\left./ \mathrm{m}^{2}\right)$. Error bars represent standard error of the mean seed output $(n=40)$. 

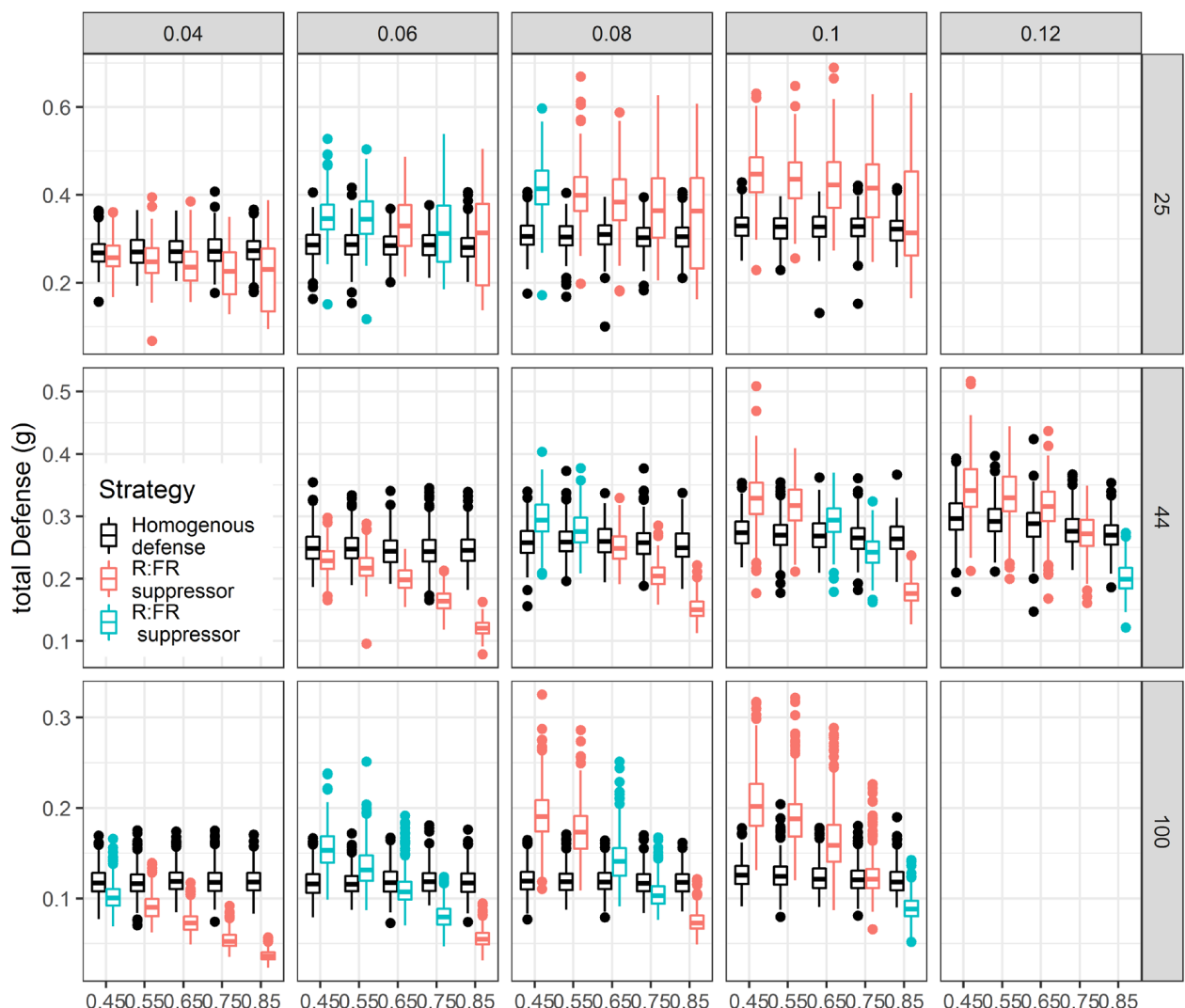

Midpoint of $\mathrm{R}: \mathrm{Fr}$ response curve $\left(\mathrm{R}: \mathrm{Fr}_{0}\right)$

Figure S5.2. The total amount of invested in defence for the homogenously defending strategy (black) and the by R:FR defence suppressing strategy (red and blue). Red color indicates that the strategy has a lower fitness compared to the homogeneous defender, while blue indicates a strategy with an average higher fitness compared to the homogeneous defender (see fig S1). The midpoints (x-axis) and the maximum (columns) of the defence-suppression curves are varied, in two densities (rows; plants $/ \mathrm{m}^{2}$ ). 

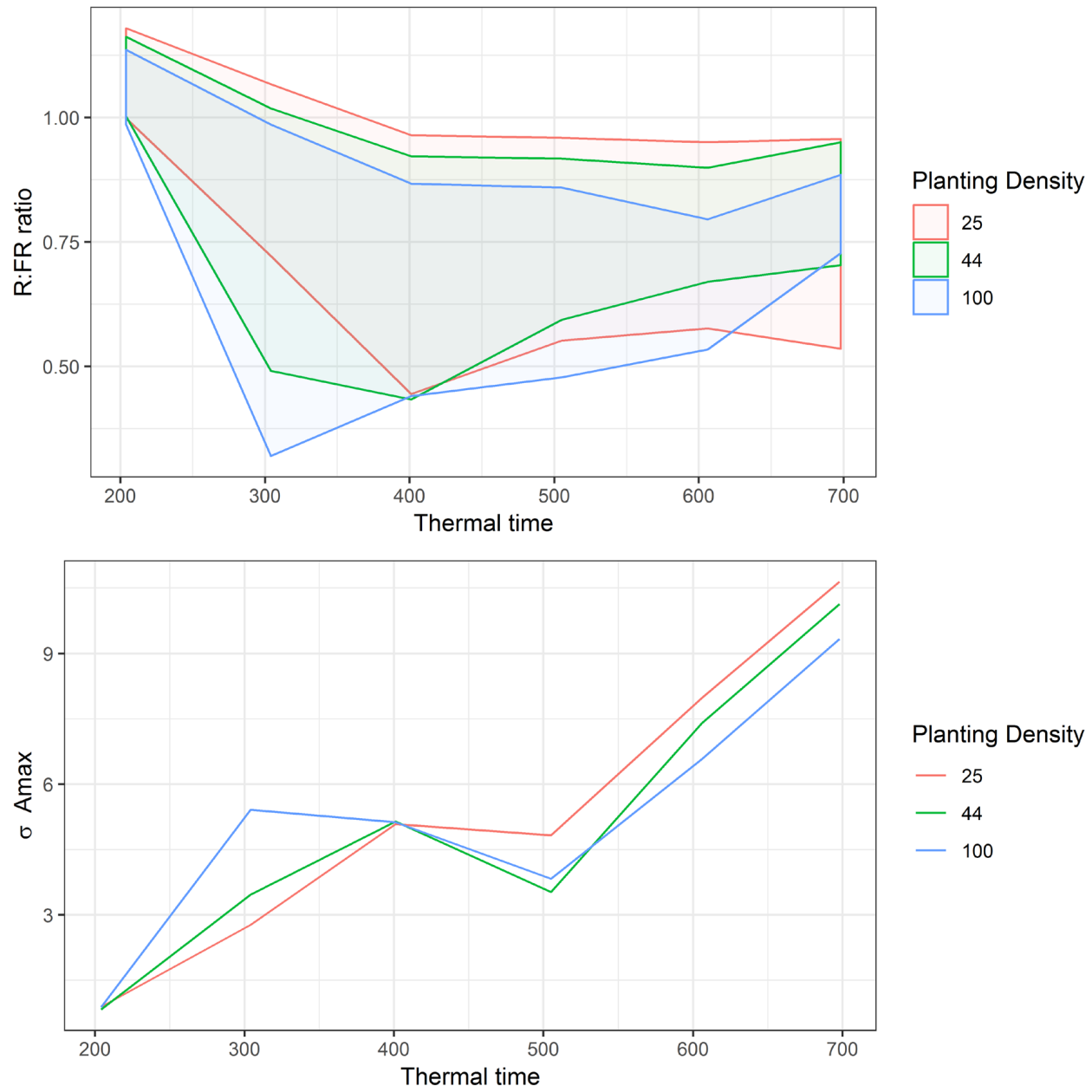

Figure S5.3. Relationship between thermal time and the range in R:FR ratio (upper $95 \%$ and lower $5 \%$ quantile) perceived by leafs in three different planting densities (upper panel), and the standard deviation in Amax. 

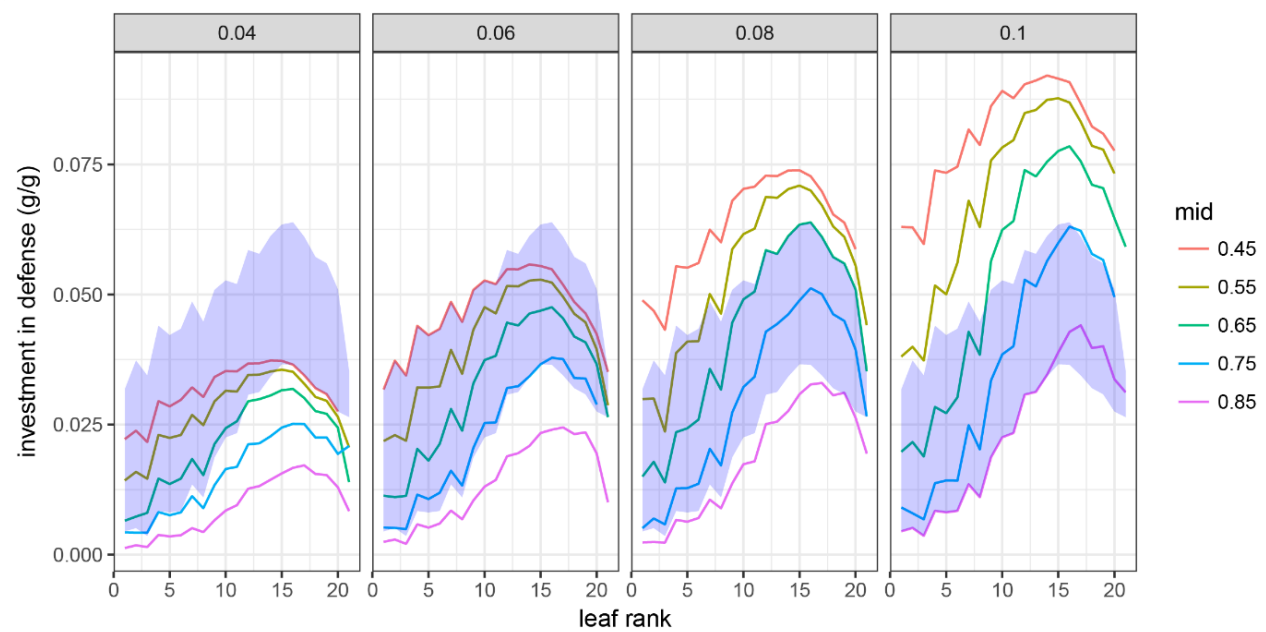

Figure S5.4. The relationship between leaf rank and the investment in defence ( $\mathrm{g} / \mathrm{g}$ leaf) for a plant density of 0.1 plants $/ \mathrm{m}^{2}$ and different $\mathrm{D}_{\max }$ (columns) and different midpoints (R:FR colours) of plants at day 80 of the growing season. The blue shaded area indicates the combination of $\mathrm{D}_{\max }$ and $\mathrm{R}: \mathrm{FR}_{0}$ that results in a higher fitness compared to a plant that does not suppress its defence in response to R:FR. 



\section{Chapter 6}

Simulation of optimal defence against herbivores under resource limitation and competition using an evolutionary functionalstructural plant model

\footnotetext{
Jorad de Vries ${ }^{1,2 *}$, Jochem B. Evers ${ }^{2}$, Erik H. Poelman ${ }^{1}$ and Niels P.R. Anten ${ }^{2}$

${ }^{1}$ Wageningen University, Laboratory of Entomology, Wageningen, The Netherlands

${ }^{2}$ Wageningen University, Centre for Crop System Analysis, Wageningen, The Netherlands
} 


\title{
Chapter 6
}

\begin{abstract}
Plants compete with their neighbours for limited resources such as light and nitrogen. This competition may drive selection towards a strong investment in competitive ability at the expense of investment in other functions such as defence against insect herbivory. The impact of this trade-off between competitive ability and defence on plant fitness is strongly influenced by dynamic interactions with environmental factors. However, understanding how defence traits impact plant fitness through this ecological network of interactions remains one of the challenges in the field of plant evolutionary ecology. In this study we aim to elucidate how the interactions between competition for light and nitrogen, soil nitrogen availability and herbivory drive selection on the balance between the acquisition and protection of resources. To this end we developed a novel simulation model that combines functional-structural plant (FSP) modelling of plant growth in a 3D light climate with a model of evolution that drives biomass allocation patterns. From the mechanisms in the model emerge the functional equilibria described by the optimal partitioning theory (OPT), which predicts resource limitation to increase biomass allocation towards plant parts that aid in acquiring that resource. Our results further show that the optimal level of defence negatively correlates with nitrogen availability and plant density at a fixed availability of nitrogen per plant, and positively correlates with plant density at a fixed availability of nitrogen per unit area. We conclude that the adaptive value of plant defence is dependent on the availability of, and competition for both above- and belowground resources.
\end{abstract}




\section{Introduction}

For many plant species, outcompeting neighbouring plants for resources such as light and nitrogen is vital to plant fitness. Therefore, plants have evolved strong plastic responses to increase their competitive ability in the presence of neighbouring plants, both above- (Ballaré $\&$ Pierik, 2017) and belowground (Mommer et al., 2012). These plastic responses function to optimise resource acquisition in environments that vary within and between generations (Sultan, 2000). However, game-theoretical studies show that under competition for resources, natural selection can drive the expression of resource acquisition traits away from the population level optimum, also referred to as a tragedy of the commons (Falster \& Westoby, 2003; McNickle \& Dybzinski, 2013). A key element in this theory is that plant fitness is not determined by the absolute value of a trait, but its relative value compared to the trait values of neighbouring plants (McNickle \& Dybzinski, 2013). This can lead to overexpression of traits that promote the acquisition of limiting resources, which has been shown both above- (King, 1990; Oksanen, 1990; Zhang et al., 1999; Anten, 2002; Falster \& Westoby, 2003; Dybzinski et al., 2011) and belowground (Givnish, 1995; Gersani et al., 2001; O'Brien et al., 2005; O'Brien et al., 2007; Dybzinski et al., 2011), although the latter is contested (Chen et al., 2015).

This investment in competitive ability may go at the expense of investment in other functions. The expression of traits related to competitive ability against neighbouring plants and defence against herbivores constitute a well-known example of such an internal resource trade-off (Züst \& Agrawal, 2017), which manifests as species-specific strategies or plastic responses to these environments (Herms \& Mattson, 1992; Züst et al., 2011; Ballaré, 2014; Züst $\&$ Agrawal, 2017). Plants use the red to far-red ratio (R:FR) in the light spectrum to detect neighbour presence (Ballaré et al., 1990), which elicits a range of growth-related responses (Franklin, 2008; Ballaré \& Pierik, 2017) as well as physiological responses that impede the plant's ability to defend itself (Herms \& Mattson, 1992; Ballaré, 2014; Züst \& Agrawal, 2017). Defensive mechanisms carry substantial metabolic costs (Gershenzon, 1994; Bekaert et al., 2012) that impact plant fitness under certain conditions such as resource limitation and competition for resources (Heil, 2002; Koricheva, 2002; Strauss et al., 2002; Cipollini et al., 2014). Plants are known to minimise the costs of an investment in defence by varying the defence investment in different organs proportional to the value of each organ (Koricheva \& Barton, 2012). The value of an organ is dependent on its position relative to local resource abundance, as well as on the investment of limiting resources in the organ. The investment of one limiting resource in organ growth can be determined by the presence of another important 
resource, as is the case with nitrogen and light. Plants are known to distribute nitrogen in the canopy following the light gradient to optimise photosynthetic gain (Hirose et al., 1987; Anten et al., 1995; Hikosaka et al., 2016). However, increased nitrogen investment that optimises the photosynthetic gain of leaves that experience high light conditions also makes these leaves prime targets for the herbivores attacking the plant (Feeny, 1976; Cates, 1980; Schoonhoven et al., 2005), which incentivises plants to express high levels of defence in these leaves (Koricheva $\&$ Barton, 2012). The impact of defence traits on plant fitness is strongly influenced by dynamic interactions with biotic and abiotic factors in the environment such as resource availability, competition pressure and herbivore community composition (Heil \& Baldwin, 2002; Strauss et al., 2002; Cipollini et al., 2014; de Vries et al., 2017). However, understanding how defence traits impact plant fitness through this ecological network of interactions and drive selection for optimally competing and defending phenotypes remains one of the current challenges in the field of plant evolutionary ecology (Poelman, 2015; Erb, 2018).

In this study, we aim to elucidate how competition for light and nitrogen, soil nitrogen availability and herbivore damage drive selection on the balance between the acquisition and protection of resources. To this end, we developed a novel functional-structural plant (FSP) model of plant growth in a 3D light climate and applied it using an evolutionary algorithm. Analytical models of evolutionary game theory (EGT) (McNickle \& Dybzinski, 2013) and three-dimensional models of plant growth (Evers et al., 2018) have existed for decades, yet merging of these two approaches has been suggested (Bongers et al., 2014; Bongers et al., 2018) but sparsely realised (Renton \& Poot, 2014; Yoshinaka et al., 2018). These examples of evolutionary FSP models are highly simplified from a mechanistic point of view, lacking the dynamic interaction between plants as they compete for resources. In analytical models of EGT, the adaptive value of a given strategy (trait expression) depends on how the performance level of a plant is affected by the strategy of neighbouring plants. In these analytical models, the interactions between plants through resource competition, signalling or other means are usually based on implicit assumptions. In contrast, FSP models can simulate these interactions explicitly since in FSP models plants are represented as individual entities that interact with one another as they grow over time. We used this merger of modelling approaches to investigate how a combination of three environmental factors (plant density, nitrogen availability and herbivory) affect i) biomass allocation patterns, ii) defence expression and iii) the distribution of nitrogen and defence in the canopy. 

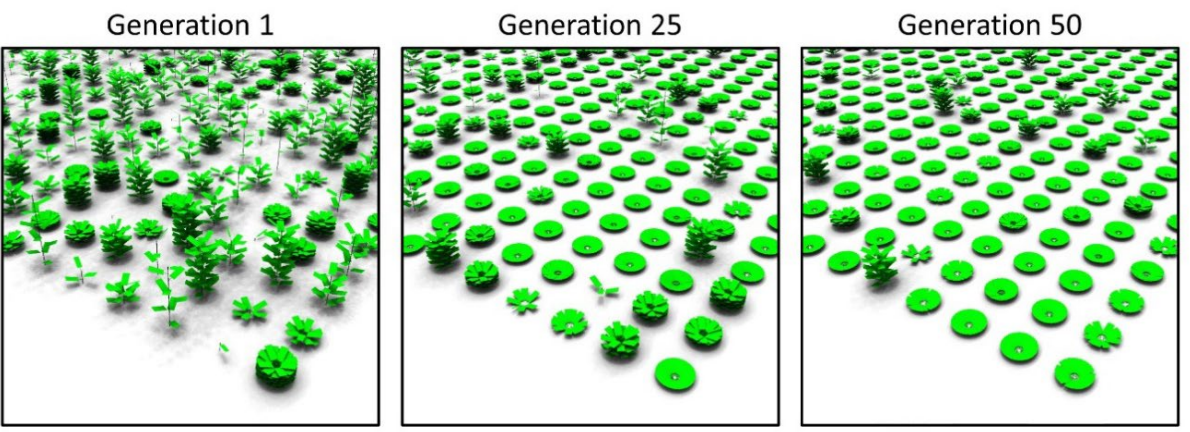

Figure 6.1 A visual representation of initial population (generation 1) of randomly generated genotypes and the population after 25 and 50 generations of evolution in a low density, low nitrogen environment in the absence of herbivory.

\section{Methods}

The model used in this study was designed to simulate the evolution of a population of plants over 75 generations using a three-dimensional (3D) FSP modelling approach (Vos et al., 2010), implemented in the platform GroImp v1.5 (Hemmerling et al., 2008). We chose to use a highly simplified plant growth model containing only the essential mechanisms to simulate competition for multiple resources above- and belowground. This choice was made to maximise the computational efficiency of the model and to keep the output comprehensible. The model simulates a population of 100 randomly generated plant genotypes facing different plant densities, levels of nitrogen availability and herbivore damage. This population is simulated over 75 generations, allowing the population to converge to a genotype that maximises fitness for the environmental conditions of the treatment combination. The model allows to assess how these environmental factors drive the allocation of biomass to leaves, stems, roots and defence as well as the distribution of nitrogen and defence in the canopy to maximise plant fitness. The model simulated the distribution of light interception in the canopy and, therefore, the outcome of competition for light between individual plants from an explicitly represented canopy structure. This canopy structure was described by the architectural characteristics of the individual plants such as plant height and number of leaves, and the density at which these plants grew (Figure 6.1). The plants were represented as simple as possible to maintain the computational efficiency needed for an evolutionary model, while still containing the essential characteristics needed to be able to grow and compete for light and nitrogen. The distribution 


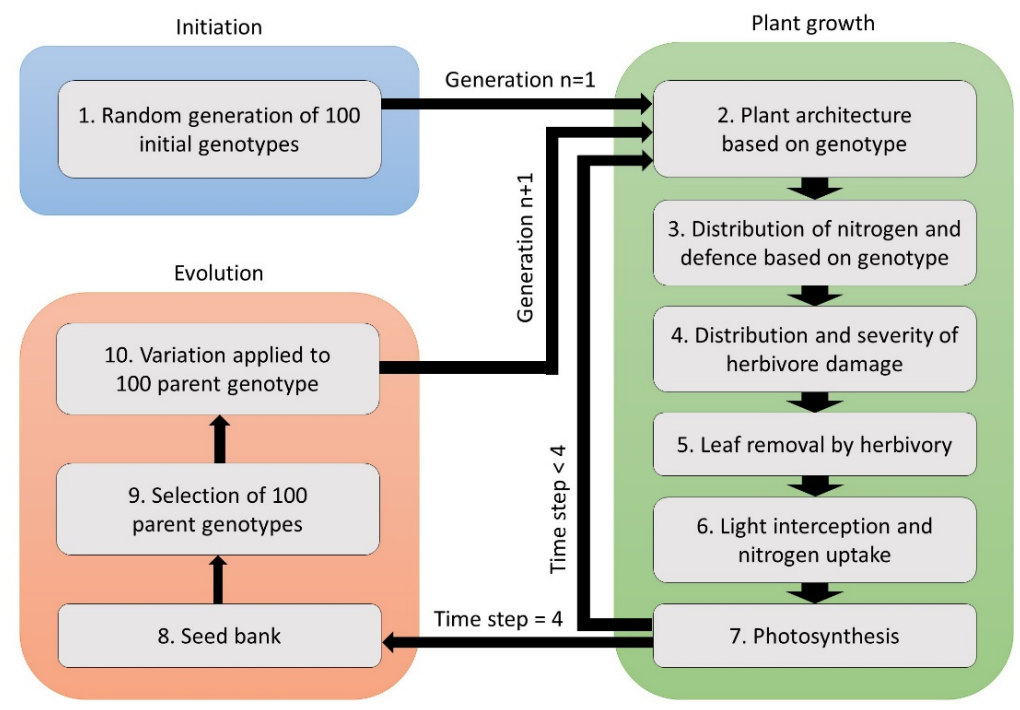

Figure 6.2 Flow chart of the model showing the steps involved in the initiation of the first population of 100 random genotypes (1), the plant growth part of the model that eventually determines plant fitness (2-7) and the evolutionary part of the model that determines how plant fitness translates to the next generation (8-10).

of light interception in the 3D-scene was simulated using a Monte-Carlo pathtracer embedded in GroImp (Hemmerling et al., 2008). The light environment was modelled using both randomly arranged diffuse light sources and direct light sources spread over the solar path at the height of summer (June $21^{\text {st }}$ ) at a latitude corresponding to The Netherlands (Evers 2010). Plots of simulated plants were replicated 25 times in the $\mathrm{x}$ and $\mathrm{y}$ directions for the light model calculations. The light conditions experienced by the 625 copies of each individual plant were then averaged, and this procedure effectively eliminated border effects on light interception. Nitrogen capture was simulated using a limited pool of soil nitrogen shared among plants, from which plants take up nitrogen proportionately to their investment in root biomass.

\section{Model summary}

The simulated plants were described using a genotype, which was characterised by four biomass allocation parameters (roots, stem, leaves and defence (e.g. secondary metabolites that can then be distributed over the plant)) and two distribution parameters for nitrogen and defence within a plant. A genotype of a given combination of values for these six allocation and distribution parameters thus represented a strategy in a game theoretical context. In every generation, plants started with five grams of biomass (e.g. carbon based assimilates) that was then divided among 
roots, stem, leaves and defence according to the biomass allocation parameters of the genotype. More biomass was accumulated over four model time steps spanning 14 days each. We chose this number of time steps balancing simulation time and accuracy (Figure S6.1). In each time step, the following calculations were made (Figure 6.2):

1. An initial population was established through random generation of the values for the allocation and distribution parameters for 100 genotypes.

2. The plant architecture was constructed by distributing the total plant biomass over leaves, stem, roots and defence using the four biomass allocation parameters.

3. Nitrogen and defences were distributed over the leaves according to the two distribution parameters.

4. The distribution and severity of herbivore damage was calculated based on the distribution of nitrogen and defence.

5. Reduced leaf biomass due to herbivory was calculated as well as the defence biomass and nitrogen present in the damaged leaf as these were consumed with the leaf.

6. Plant nitrogen uptake and light interception were calculated based on the plant architecture after herbivory.

7. Amounts of light and nitrogen captured was used to simulate carbon assimilation through photosynthesis, the maximum rate of which was dependent on the photosynthetically active nitrogen in a leaf. The carbon assimilated through photosynthesis was added to the total plant biomass to conclude one time step.

Steps 2-7 were repeated for a total of four time steps for every generation.

8. Finally, each genotype contributed the assimilates produced during the fourth time step to a virtual seed bank rather than to the total plant biomass.

9. A new population of genotypes was constructed by randomly drawing 100 genotypes from the seed bank, with the chance for a genotype to be drawn being proportionate to its presence in the seed bank.

10. The plants of the next generation inherit the genotypes randomly drawn from the seed bank with a fixed range of variation applied to all parameters that make up those genotypes.

This was repeated for 75 generations and ten replications were simulated per treatment combination. The components of the plant growth and evolutionary parts of the model and 
are explained in detail below.

\section{Plant architecture}

The model implements a highly simplified description of plant architecture that minimises computational demand while maintaining the essential characteristics to simulate competition for light and nitrogen. The plants consist of a single, branchless stem with rectangular leaves of a consistent size located equidistantly along the stem. The leaves are attached to the stem with a petiole of a fixed length (PLen, $\mathrm{m}$ ), with a consistent insertion angle (LAngle, degrees from vertical) and follow a spiral phyllotaxis (Phyl, degrees) along the length of the stem. The roots are described using root biomass only, disregarding any root architectural characteristics such as rooting depth, root mass density and specific root length. The model assumes no loss of biomass due to leaf senescence, root turnover or maintenance respiration.

The model also assumes no plastic responses to the environment such as shade avoidance responses or changes in specific leaf area, meaning that growth is the only mechanism through which plant architecture changes over time.

\section{Biomass allocation}

The biomass allocated to a plant tissue (Bio $o_{t, i}, t=l e a f$, stem,root,def, $\mathrm{g}$ ) is determined by the total plant biomass $\left(\mathrm{Bio}_{\mathrm{i}}, \mathrm{g}\right)$ and the biomass allocation parameter of the plant genotype corresponding to the plant tissue $\left(A_{t}, t=l e a f\right.$, stem,root,def $)$.

$$
\mathrm{Bio}_{t, i}=A_{t} * \mathrm{Bio}_{i}
$$

\section{Leaves}

For simplification purposes, the simulated leaves had a fixed length, so that the total number of leaves on a plant (NLeaves ${ }_{i}$ ) could be calculated from the plant's total leaf biomass (Bioleaf,i, g), and the parameters for leaf length (LLen, $\mathrm{m}$ ), leaf width:length ratio (WLR), and specific leaf area $\left(\right.$ SLA, $\left.\mathrm{m}^{2} \mathrm{~g}^{-1}\right)$.

$$
\text { Nleaves }_{i}=\frac{\text { Bio }_{\text {leaf }, i} * S L A}{{L L e n^{2}}^{2} W L R}
$$

Because this value would likely not be a integer, the top leaf of each plant had a reduced width (Width ${ }_{\mathrm{L}, \mathrm{top}}$, in $\mathrm{m}$ ), which was calculated using the plant's total number of leaves as an integer $\left(\mathrm{NrL}_{\mathrm{i}}\right)$. This ensured a discrete number of leaves and that all the leaf biomass was converted to leaf area. 


$$
\text { Width }_{L, t o p}=\left(\text { Nleaves }_{i}-N r L_{i}-1\right) * L L e n * W L R
$$

\section{Stem}

The stem length of a plant (StemLength ${ }_{i}$, in $\mathrm{m}$ ) was calculated by the plant's stem biomass $\left(\right.$ Bio $_{\text {stem, },}$, in $\mathrm{g}$ ) and a specific stem length parameter $\left(\mathrm{SSL}, \mathrm{m} \mathrm{g}^{-1}\right)$.

$$
\text { StemLength } h_{i}=\text { Bio }_{\text {stem }, i} * S S L
$$

The distance between the leaves on the plant (InternodeLength, $\mathrm{m}$ ) was then calculated by dividing the stem length by the number of leaves, resulting in leaves being positioned equidistantly along the stem.

$$
\text { InternodeLengt }_{i}=\frac{\text { StemLengt }_{i}}{\text { Nleaves }_{i}}
$$

\section{Roots and nitrogen}

The potential nitrogen uptake of a plant $\left(\mathrm{Nupt}_{\text {pot,i, }}\right.$, in $\left.\mathrm{g} \mathrm{day}^{-1}\right)$ was calculated using a nitrogen uptake rate parameter $\left(\mathrm{N}_{\mathrm{rt}}, \mathrm{g}_{\text {day }}{ }^{-1} \mathrm{~g}^{-1}\right.$ root DW, (Siddiqi et al., 1990; Malagoli et al., 2004)) and the plant's root biomass (Bioroot, in $\mathrm{g}$ ).

$$
N u t_{p o t, i}=N_{r t} * \text { Bio }_{\text {root }, i}
$$

The realised nitrogen uptake was added to the plant nitrogen pool $\left(\mathrm{dN}_{\mathrm{i}} / \mathrm{dt}\right.$, in $\left.\mathrm{g} \mathrm{day}^{-1}\right)$ and could be limited by 1) nitrogen availability if the sum of potential nitrogen uptake of all plants exceeds the available soil nitrogen $\left(\sum_{i=1}^{n} N u p t_{p o t, i}>\right.$ Nsoil), or 2) potential nitrogen uptake rate of the plant if the soil nitrogen availability was sufficient to satisfy the sum of potential nitrogen uptake of all plants $\left(\sum_{i=1}^{n} N u p t_{p o t, i}<\right.$ Nsoil). If soil nitrogen was limiting uptake, the available soil nitrogen (Nsoil, in g) was distributed over the plants based on their root biomass (Bioroot,i, in $\mathrm{g}$ ) compared to the total root biomass of all plants $\left(\sum_{i=1}^{n}\right.$ Bio $_{\text {root }, i}$, in $\left.\mathrm{g}\right)$.

$$
\frac{d N_{i}}{d t}=N_{\text {soil }} * \frac{\text { Bio }_{\text {root }, i}}{\sum_{i=1}^{n} \text { Bio }_{\text {root }, i}} \quad \text { if } \quad \sum_{i=1}^{n} N u p t_{\text {pot }, i}>N_{\text {soil }}
$$




$$
\frac{d N_{i}}{d t}=\text { Nupt }_{\text {pot }, i} \quad \text { if } \quad \sum_{i=1}^{n} N u p t_{\text {pot }, i}<N_{\text {soil }}
$$

The nitrogen in the plant was then distributed over its leaves based on the relative nitrogen value of a leaf $\left(\mathrm{rN}_{\mathrm{L}}\right)$ (indicating the leaf nitrogen investment relative to other leaves) that was calculated from the plant's nitrogen distribution parameter $\left(\mathrm{nDis}_{\mathrm{i}}\right)$, the leaf rank $\left(\mathrm{rank}_{\mathrm{L}}\right)$ and the total number of leaves on the plant $\left(\mathrm{NrL}_{\mathrm{i}}\right)$.

$$
r N_{L}=\frac{1}{1+\exp \left(10 *\left(n D i s_{i}-\left(1-n D i s_{i}\right)\right) *\left(\frac{\operatorname{rank}_{L}}{N r L_{i}}-0.5\right)\right)}
$$

This function describes the distribution of nitrogen as a sigmoid that is skewed towards low ranked (old) leaves at a low value of $\mathrm{nDis}(<0.5)$ and high ranked (young) leaves at a high value of $\mathrm{nDis}(>0.5)$. At an $\mathrm{nDis}$ of 0.5 , the function describes a horizontal line, representing an equal distribution of nitrogen over the leaves in the canopy.

The amount of nitrogen allocated to the leaves $\left(\mathrm{N}_{\text {leaf }, \mathrm{i}}, \mathrm{g}\right)$ was calculated from the total plant nitrogen $\left(\mathrm{N}_{\mathrm{i}}, \mathrm{g}\right)$, the minimum plant nitrogen requirement $\left(\mathrm{N}_{\min }, \mathrm{g} \mathrm{N} \mathrm{g}^{-1}\right.$ biomass, (Yin \& Laar, 2005)) and the biomass allocated to roots, stem and defence.

$$
N_{\text {leaf }, i}=N_{i}-N_{\text {min }} *\left(\text { Bio }_{\text {root }, i}+\text { Bio }_{\text {stem }, i}+\text { Bio }_{\text {def }, i}\right)
$$

The amount of nitrogen allocated to an individual leaf $\left(\mathrm{N}_{\mathrm{L}}, \mathrm{g}\right)$ was then calculated from the total nitrogen allocated to leaves $\left(\mathrm{N}_{\text {leaf, }, \mathrm{i}} \mathrm{g}\right)$ and the leaf relative nitrogen value compared to the sum of all relative leaf nitrogen values within the plant $\left(\sum_{L=1}^{n} r N_{L}\right)$.

$$
N_{L}=N_{\text {leaf }, i} * \frac{r N_{L}}{\sum_{L=1}^{n} r N_{L}}
$$

\section{Defence and herbivory}

The biomass allocated to defence was distributed over the leaves based on the relative defensive value of a leaf $\left(\mathrm{rD}_{\mathrm{L}}\right)$ (indicating how well a leaf is defended relative to other leaves) that was calculated from the plant's defence distribution parameter $\left(\mathrm{dDis}_{\mathrm{i}}\right)$, the leaf rank $\left(\operatorname{rank}_{\mathrm{L}}\right)$ and the 
total number of leaves on the plant $\left(\mathrm{NL}_{\mathrm{i}}\right)$.

$$
r D_{L}=\frac{1}{1+\exp \left(10 *\left(d D i s_{i}-\left(1-d D i s_{i}\right)\right) *\left(\frac{r a n k_{L}}{N L_{i}}-0.5\right)\right)}
$$

The amount of defences allocated to a leaf $\left(\mathrm{D}_{\mathrm{L}}, \mathrm{g}\right)$ was then calculated from the plant defence biomass $\left(\right.$ Bio $\left._{\text {def,i, }}, \mathrm{g}\right)$ and the leaf's relative defensive value compared to the total leaf defensive value of the plant $\left(\sum_{L=1}^{n} r D_{L}\right)$.

$$
D_{L}=B i o_{d e f, i} * \frac{r D_{L}}{\sum_{L=1}^{n} r D_{L}}
$$

The amount of herbivore damage was dependent on the leaf defence concentration $\left(\mathrm{cD}_{\mathrm{L}}\right)$, which was calculated with the biomass of a single leaf $\left(\mathrm{Bio}_{\mathrm{L}}, \mathrm{g}\right)$ and defences allocated to that leaf.

$$
c D_{L}=\frac{D_{L}}{B i o_{L}}
$$

The herbivore distribution within the plant $\left(\mathrm{hDis}_{\mathrm{i}}, 0-1\right)$ was determined by the distribution of nitrogen (nDis, $0-1)$ and defence (dDis, $0-1)$ in the canopy so that the herbivores were attracted to leaves with high nitrogen and repelled by leaves with high defences.

$$
h D i s_{i}=\frac{1}{2}\left(n D i s_{i}+\left(1-d D i s_{i}\right)\right)
$$

The potential damage done to a leaf $\left(\mathrm{Pdmg}_{\mathrm{L}}, 0-1\right)$ was calculated using a herbivore damage coefficient ( $\mathrm{h}$; 0-0.3 in treatments), the herbivore distribution within the plant $\left(\mathrm{hDis}_{\mathrm{i}}\right)$, the leaf rank $\left(\operatorname{rank}_{\mathrm{L}}\right)$ and the total number of leaves on the plant $\left(\mathrm{NrL}_{\mathrm{i}}\right)$ (see Figure S6.2).

$$
P d m g_{L}=\frac{2 * h}{1+\exp \left(10 *\left(h D i s_{i}-\left(1-h D i s_{i}\right)\right) *\left(\frac{r a n k_{L}}{N r L_{i}}-0.5\right)\right)}
$$

The relative damage done to a leaf $\left(\mathrm{dmg}_{\mathrm{L}}, 0-1\right)$ was then calculated using the potential damage done to the leaf $\left(\mathrm{Pdmg}_{\mathrm{L}}\right)$, the maximum damage reduction by defence $\left(\mathrm{R}_{\max }\right)$ and the value of 
leaf defence concentration at which half of the maximum damage reduction was realised $(\mathrm{Kr}$, $\mathrm{g}$ defence $\mathrm{g}^{-1}$ leaf biomass).

$$
d m g_{L}=P d m g_{L} *\left(1-\frac{R_{\max } * c D_{L}}{c D_{L}+K r}\right)
$$

Herbivore damage reduced the biomass ( $\left.\mathrm{Bio}_{\mathrm{L}}, \mathrm{g}\right)$, width $\left(\mathrm{Width}_{\mathrm{L}}, \mathrm{m}\right)$, and with that the defence $\left(\mathrm{D}_{\mathrm{L}}, \mathrm{g}\right)$ and nitrogen content $\left(\mathrm{N}_{\mathrm{L}}, \mathrm{g}\right)$ of the damaged leaf was also reduced.

$$
\begin{aligned}
\frac{d B i o_{L}}{d t} & =- \text { Bio }_{L} * d m g_{L} \\
\frac{d W i d t h_{L}}{d t} & =-W i d t h_{L} * d m g_{L} \\
\frac{d D_{L}}{d t} & =-D_{L} * d m g_{L} \\
\frac{d N_{L}}{d t} & =-N_{L} * d m g_{L}
\end{aligned}
$$

These reductions were taken into account for the calculations in the next time step by also reducing plant variables of leaf biomass, defence and nitrogen.

$$
\begin{gathered}
\frac{d \text { Bio }_{\text {leaf }, i}}{d t}=-\sum_{L=1}^{n}\left(\text { Bio }_{L} * d m g_{L}\right) \\
\frac{d B i o_{d e f, i}}{d t}=-\sum_{L=1}^{n}\left(D_{L} * d m g_{L}\right) \\
\frac{d N_{i}}{d t}=-\sum_{L=1}^{n}\left(N_{L} * d m g_{L}\right)
\end{gathered}
$$

\section{Photosynthesis}

Leaf photosynthesis was calculated using a light response curve that was dependent on the leaf nitrogen content ( $\mathrm{cN}_{\mathrm{L}}, \mathrm{g} \mathrm{N} \mathrm{g}^{-1}$ leaf biomass), which was calculated using leaf nitrogen $\left(\mathrm{N}_{\mathrm{L}}, \mathrm{g}\right.$ $\mathrm{N})$ and leaf biomass (BioL, $\mathrm{g})$.

$$
c N_{L}=\frac{N_{L}}{B i o_{L}}
$$


The light-saturated photosynthesis of a leaf (Amax $\left.\mathrm{L}_{\mathrm{L}} \mathrm{g} \mathrm{CO}_{2} \mathrm{~m}^{-2} \mathrm{~s}^{-1}\right)$ of a leaf was dependent on the maximum rate of photosynthesis (Amax0) and the leaf nitrogen content relative to the minimum nitrogen content of a leaf required to photosynthesise $\left(\mathrm{N}_{\min }, \mathrm{g} \mathrm{N} \mathrm{g}^{-1}\right.$ biomass (Yin \& Laar, 2005)) and the nitrogen content of a leaf at which photosynthesis is maximised $\left(\mathrm{N}_{\max }, \mathrm{g}\right.$ $\mathrm{N} \mathrm{g}^{-1}$ biomass (Yin \& Laar, 2005)).

$$
\begin{array}{ccc}
\operatorname{Amax}_{L}=0 & \text { if } & c N_{L}<N_{\text {min }} \\
\operatorname{Amax}_{L}=A \max 0 * \frac{c N_{L}-N_{\text {min }}}{N_{\max }-N_{\text {min }}} & \text { if } & N_{\text {min }}<c N_{L}<N_{\text {max }} \\
\operatorname{Amax}_{L}=A \max 0 & \text { if } & c N_{L}>N_{\max }
\end{array}
$$

The leaf's gross photosynthesis rate $\left(\mathrm{Ag}_{\mathrm{L}}, \mu \mathrm{mol} \mathrm{CO} \mathrm{Cm}^{-2} \mathrm{~s}^{-1}\right)$ was calculated using the absorbed photosynthetically active radiation by the leaf $\left(\mathrm{APAR}_{\mathrm{L}}, \mu \mathrm{mol}\right)$, the initial slope of the light response curve $\left(\varepsilon, \mu \mathrm{mol} \mathrm{CO} \mathrm{CO}_{2}{ }^{-1}\right)$ and the light-saturated rate of photosynthesis of the leaf $\left(\operatorname{Amax}_{\mathrm{L}} \mathrm{g} \mathrm{CO}_{2} \mathrm{~m}^{-2} \mathrm{~s}^{-1}\right)$.

$$
A g_{L}=\operatorname{Amax}_{L} *\left(1-\exp \left(-\varepsilon * \frac{A P A R_{L}}{A \max _{L}}\right)\right)
$$

Net photosynthesis of a leaf $\left(\mathrm{An}_{\mathrm{L}}, \mu \mathrm{mol} \mathrm{CO} \mathrm{CO}^{-2} \mathrm{~s}^{1}\right)$ accounts for dark respiration $(\mathrm{Rd}, \mu \mathrm{mol}$ $\mathrm{CO}_{2} \mathrm{~m}^{-2} \mathrm{~s}^{1}$ ), which was kept constant, regardless of Amax or leaf nitrogen content.

$$
A n_{L}=A g_{L}-R d
$$

The $\mathrm{CO}_{2}$ assimilated by the leaves was converted to produce plant biomass $\left(\mathrm{Bio}_{\mathrm{i}}, \mathrm{g}\right)$. A conversion parameter $(\mathrm{C})$ converted from $\mu \mathrm{mol} \mathrm{CO}_{2}$ to grams of glucose and seconds to days, a second conversion parameter accounted for the construction costs from glucose to biomass production (cc, g glucose $\mathrm{g}^{-1}$ biomass).

$$
\frac{d B i o_{i}}{d t}=C * \frac{\sum_{L=1}^{n} A n_{L}}{c c}
$$


Table 6.1 Model parameters describing plant architecture and resource acquisition.

\begin{tabular}{|c|c|c|c|c|}
\hline Description & Name & Value & Unit (reference) & Eq. \\
\hline Petiole length & PLen & 0.075 & $\mathrm{~m}$ & \\
\hline Leaf insertion angle & LAngle & 70 & Degrees from the vertical & \\
\hline Phyllotaxis & Phyl & 137.5 & Degrees & \\
\hline Specific leaf area & SLA & 0.03 & $\mathrm{~m}^{2} \mathrm{~g}^{-1}$ & 6.2 \\
\hline Leaf length & LLen & 0.25 & $\mathrm{~m}$ & $6.2,6.3$ \\
\hline Leaf width/length ratio & WLR & 0.5 & dimensionless & $6.2,6.3$ \\
\hline Specific Stem length & SSL & 0.07 & $\mathrm{~m} \mathrm{~g}^{-1}$ & 6.4 \\
\hline Nitrogen uptake rate & $\mathrm{N}_{\mathrm{rt}}$ & 0.0672 & $\begin{array}{l}\mathrm{g} \text { day }^{-1} \mathrm{~g}^{-1} \text { root DW, (Siddiqi et al., } \\
\text { 1990; Malagoli et al., 2004) }\end{array}$ & 6.6 \\
\hline Minimum leaf nitrogen content required to photosynthesise & $\mathrm{N}_{\min }$ & 0.01 & $\mathrm{~g} \mathrm{~N} \mathrm{~g}^{-1}$ biomass (Yin \& Laar, 2005) & $6.9,6.25$ \\
\hline Maximum damage reduction by defence & $\mathrm{R}_{\max }$ & 1 & dimensionless & 6.16 \\
\hline Leaf defence concentration at which half of $R_{\max }$ was realised & $\mathrm{Kr}$ & 0.1 & $\mathrm{~g}$ defence $\mathrm{g}^{-1}$ leaf biomass & 6.16 \\
\hline Maximum rate of photosynthesis & Amax 0 & 30 & $\mathrm{~g} \mathrm{CO}_{2} \mathrm{~m}^{-2} \mathrm{~s}^{-1}$ & 6.25 \\
\hline Leaf nitrogen content at which photosynthesis is maximised & $\mathrm{N}_{\max }$ & 0.05 & $\mathrm{~g} \mathrm{~N} \mathrm{~g}^{-1}$ biomass (Yin \& Laar, 2005) & 6.25 \\
\hline Initial slope of the light response curve & $\varepsilon$ & 0.06 & $\mu \mathrm{mol} \mathrm{CO} 2 \mu \mathrm{mol}^{-1}$ & 26 \\
\hline Dark respiration & $\mathrm{Rd}$ & 1.2 & $\mu \mathrm{mol} \mathrm{CO} 2 \mathrm{~m}^{-2} \mathrm{~s}^{1}$ & 27 \\
\hline Construction costs from glucose to biomass production & $\mathrm{cc}$ & 1.5 & g glucose $\mathrm{g}^{-1}$ biomass & 28 \\
\hline
\end{tabular}

\section{Evolutionary algorithm}

The initial generation of 100 plants had randomly generated genotypes, meaning that the plants in generation-1 had widely different allocation and distribution traits. After one iteration of the plant growth model (steps 2-7, Figure 6.2), the fitness of these genotypes was defined by the assimilates produced during the last simulation step, which was added to a virtual seed bank. The chance that the genotype of a plant was represented in the next generation was proportional to its contribution to the seed bank of the current generation. Parents were then randomly drawn from the seed bank for the 100 plants in the next generation, where every offspring had one parent and one parent could potentially produce multiple offspring. The plants in the next generation inherited the genotype of their parent, with random variation applied to that genotype to represent the genetic variation otherwise acquired through recombination and mutation. The variation of the distribution parameters was fixed at 0.05 , e.g. for a parent value of 0.4 the offspring values were uniformly distributed between 0.35 and 0.45 . The variation of the allocation parameters was also fixed at 0.05 , unless the parameter was 1 ) smaller than 0.05 , in which case the variation was equal to the value of the parameter, or 2) larger than 0.95 , in which case the variation was equal to one minus the value of the parameter. This allowed the allocation parameters to approximate zero if the conditions select for it, as could be expected for defence allocation in the absence of herbivory. The seed bank was then emptied so that every generation 


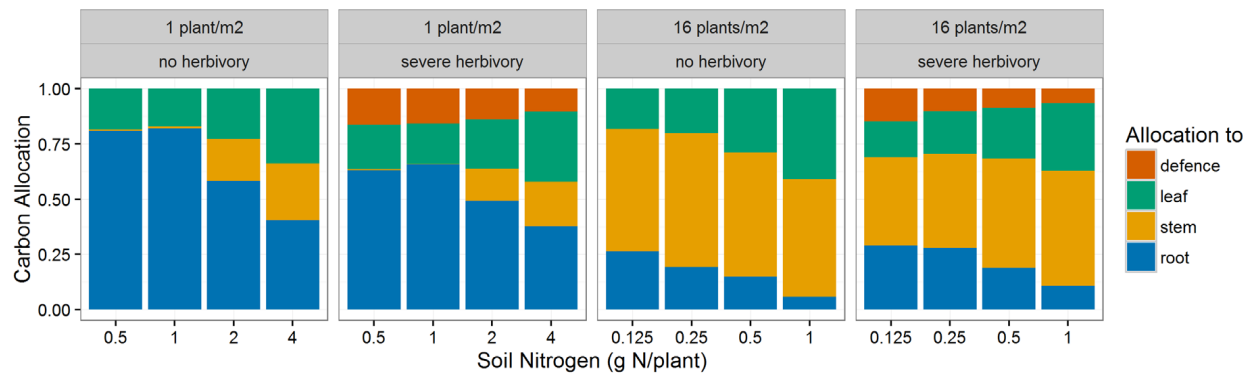

Figure 6.3 Average biomass allocated to leaf, stem and root in plant populations that evolved for 75 generations in the absence of herbivory $(\mathrm{h}=0$ in eq. 6.15) or under severe levels of herbivory ( $\mathrm{h}=0.3$ in eq. 6.15$)$, in low $(1$ plant $\mathrm{m}-2)$ or high $(16$ plant $\mathrm{m}-2)$ plant densities and different levels of soil nitrogen per plant (x-axis).

depended solely on the previous generation. This was repeated for 75 generations, which maximised computational efficiency while allowing the initial population of random genotypes to converge to a population of which the genotype was optimised to the environmental conditions presented by the treatment combination (Figure S6.3).

\section{Simulations}

Optimal genotypes were simulated for two levels of competition for light, represented by two population densities $\left(1\right.$ plant $\mathrm{m}^{-2}, 25$ plants $\left.\mathrm{m}^{-2}\right)$, a range of potential herbivore damage levels (0-0.3 with steps of 0.05 , fraction of leaf area) and four levels of soil nitrogen availability per plant density $\left(0.5,1,2\right.$ and $4 \mathrm{~g} \mathrm{~N}$ plant $^{-1}$ at 1 plant $\mathrm{m}^{-2}$ and $0.125,0.25,0.5$ and $1 \mathrm{~g} \mathrm{~N}$ plant $^{-1}$ at 16 plant $\mathrm{m}^{-2}$ ). The levels of soil nitrogen represent the nitrogen availability at the start of a growing season and are chosen such that two levels of nitrogen availability per plant are present in both densities $\left(0.5\right.$ and $1 \mathrm{~g} \mathrm{~N}$ plant $\left.^{-1}\right)$ and two levels of nitrogen availability per unit area are present in both plant densities ( 2 and $4 \mathrm{~g} \mathrm{~N} \mathrm{~m}^{-2}$, Table 6.2). When comparing the two densities the levels of nitrogen availability are shown as either the availability of nitrogen per plant $(0.5$ and $1 \mathrm{~g} \mathrm{~N}$ plant $^{-1}$ for both plant densities) or as the availability of nitrogen per unit area ( 2 and $4 \mathrm{~g} \mathrm{~N}_{\text {plant }}{ }^{-1}$ at 1 plant $\mathrm{m}^{-2}$ and 0.125 and $0.25 \mathrm{~g} \mathrm{~N}$ plant $^{-1}$ at 16 plant $\mathrm{m}^{-2}$ ). A fixed availability of nitrogen per plant simulates more fertile habitats also harbouring a higher plant density, while a fixed availability of nitrogen per unit area simulates the effect of variation in plant density at a given soil fertility level. The model output was tested for significance by conducting an analysis of variance (ANOVA) at the $5 \%$ probability level. 
Table 6.2 Nitrogen availability per plant and per unit area for the low $\left(1\right.$ plant $\left.\mathrm{m}^{-2}\right)$ and high $\left(16\right.$ plant $\mathrm{m}^{-2}$ ) plant densities.

\begin{tabular}{|c|c|c|c|c|c|}
\hline Density & g N plant ${ }^{-1}$ & g N m ${ }^{-2}$ & Density & g N plant ${ }^{-1}$ & g N m${ }^{-2}$ \\
\hline \multirow[t]{4}{*}{1 plant $\mathrm{m}^{-2}$} & 0.5 & 0.5 & 16 plant $\mathrm{m}^{-2}$ & 0.125 & 2 \\
\hline & 1 & 1 & & 0.25 & 4 \\
\hline & 2 & 2 & & 0.5 & 8 \\
\hline & 4 & 4 & & 1 & 16 \\
\hline
\end{tabular}

\section{Results}

\section{Carbon allocation to stems, leaves and roots}

To first illustrate the capacity of the model to find optimized genotypes with selection for biomass allocation traits as an emergent property, simulations were done in the absence of herbivory (Figure 6.3). This lack of herbivore pressure selected for plant genotypes that didn't invest in defence. The biomass allocation patterns show that an increase in plant density had a positive effect on the allocation to stems, resulting in taller plants, and a negative effect on the allocation to roots and leaves (Figure 6.3). This resulted in high-density plants having considerably lower root-shoot ratios than low-density plants. Plants invested less in roots with an increase in nitrogen availability (Figure 6.3), which increased the allocation to stems and leaves (Figure 6.3). These carbon allocation patterns persisted in plants subjected to herbivores, which increased their investment to defence (Figure 6.3).

\section{Allocation to defence}

Plant defence investment increased with increasing herbivore damage coefficient $h$ (Figure 6.4) and decreased with an increase in the availability of nitrogen per plant (Figure 6.4). When comparing the defence investment between plant densities, it is important to consider the way density affects the nitrogen availability to the plant. A fixed availability of nitrogen per plant (Figure 6.5a,b) simulates more fertile habitats also harbouring a higher plant density, while a fixed availability of nitrogen per unit area (Figure 6.5c,d) simulates the effect of variation in plant density at a given level of nitrogen availability. When the nitrogen availability per plant remains constant, the results showed a decrease in the optimal defence investment with an increase in plant density (Figure 6.5a,b). Conversely, when the nitrogen availability per unit 


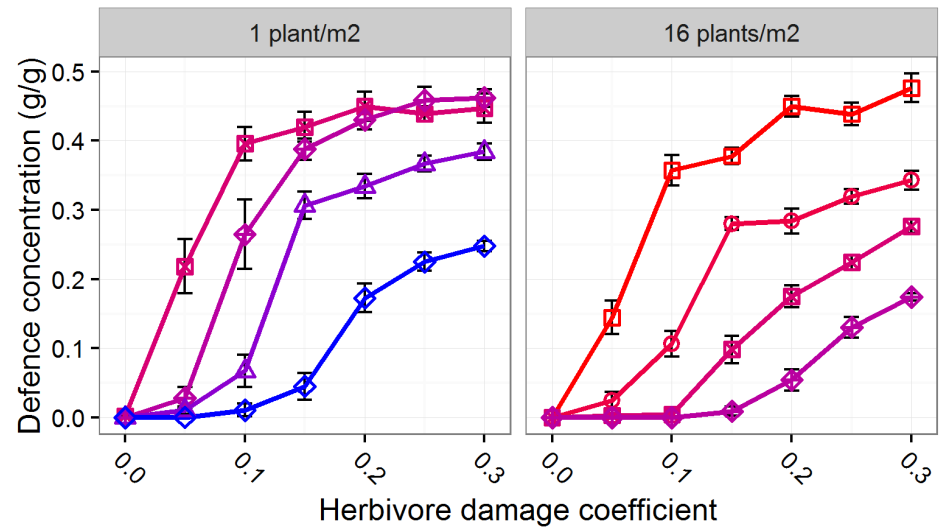

Nitrogen content

$\square \mathrm{N} 1: 0.125 \mathrm{~g} \mathrm{~N} /$ plant

N2: $0.25 \mathrm{~g} \mathrm{~N} /$ plant

N3: $0.5 \mathrm{~g} \mathrm{~N} /$ plant

$\uparrow \mathrm{N} 4: 1 \mathrm{~g} \mathrm{~N} /$ plant

$\triangle \mathrm{N} 5: 2 \mathrm{~g} \mathrm{~N} /$ plant

N6: $4 \mathrm{~g} \mathrm{~N} /$ plant

Figure 6.4 Average leaf defence concentration (grams of defence / grams of leaf mass) of plant populations that evolved for 75 generations in under low $\left(1\right.$ plant $\left.\mathrm{m}^{-2}\right)$ or high $\left(16\right.$ plant $\left.\mathrm{m}^{-2}\right)$ plant densities and difference levels of herbivore damage (x-axis, h in eq. 6.14), and a range of nitrogen contents (low $=$ red, high=blue).

area remains constant, the results showed an increase in the optimal defence investment with an increase in plant density (Figure 6.5c,d).

\section{Distribution of nitrogen and defence}

The model selected for an even distribution of nitrogen in the canopy in a low plant density, indicated by an average nDis value of 0.44 (eq. 6.8) and a peak in the frequency distribution around a nDis value of 0.5 (Figure 6.6). The model selected for a nitrogen distribution skewed towards young leaves in a high plant density, indicated by an average nDis value of 0.30 (eq. 6.8) and a narrow peak in the frequency distribution at nDis values around 0.30 (Figure 6.7). These nitrogen distributions were consistent at all levels of herbivore damage $(\mathrm{h}=0-0.3$, eq. 6.15 ) and both with a fixed availability of nitrogen per plant (Figure 6.6a,b) and a fixed availability of nitrogen per unit area (Figure 6.6c,d). The model did not select for a given distribution of defence in the canopy in the absence of herbivore damage as the plants did not invest in defence (Figure S6.4). In the presence of herbivore damage, the optimal defence distribution was unaffected by the level of herbivore damage $(\mathrm{F}=1.5, \mathrm{P}=0.21)$, but strongly affected by the plant density (Figure 6.7). The model selected for an even distribution of defences in a low plant density, indicated by an average dDis value of 0.45 (eq. 6.11) and a peak in the frequency distribution around a dDis value of 0.5 (Figure 6.7). The model selected for a defence distribution skewed towards young leaves in a high plant density, indicated by an average dDis value of 0.25 (eq. 6.11) and peaks in the frequency distribution at dDis values 


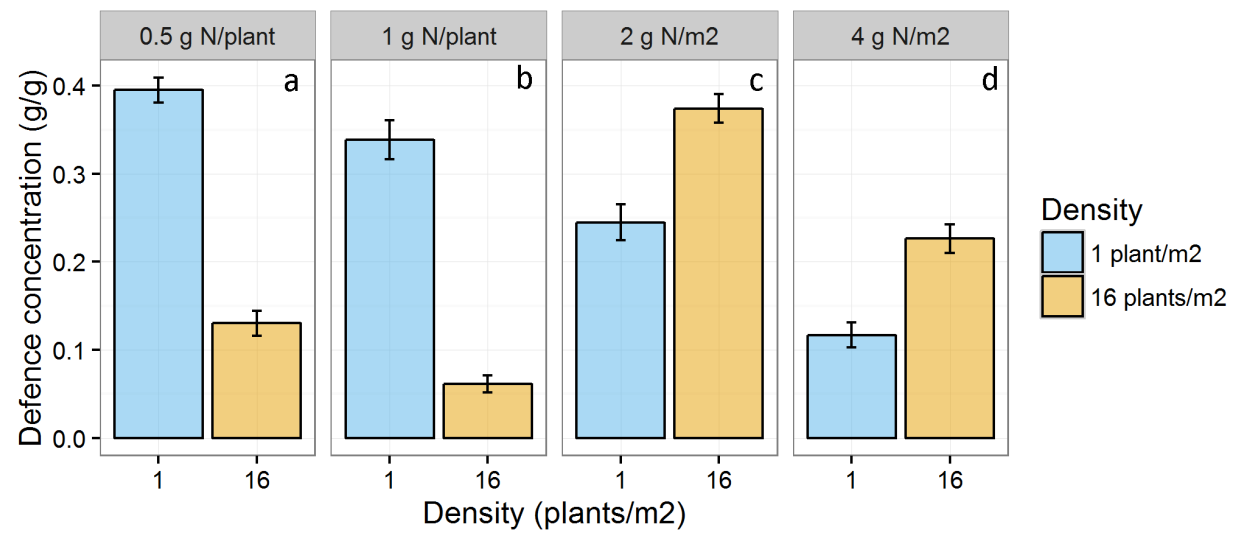

Figure 6.5 Average leaf defence concentration (grams of defence / grams of leaf mass) of plant populations that evolved for 75 generations in low (1 plant $\mathrm{m}-2)$ or high (16 plant $\mathrm{m}-2)$ plant densities (x-axis) with a fixed soil nitrogen content per plant $(a, b)$ or a fixed soil nitrogen content per unit area $(\mathrm{c}, \mathrm{d})$.

below 0.5 (Figure 6.7). The effects of density on the distribution of defence were consistent with a fixed availability of nitrogen per plant (Figure 6.7a,b) and per unit area (Figure 6.7c,d), which contrasts the effects of density and nitrogen availability on the optimal defence investment. The frequency distributions of the defence distribution parameter showed a large spread (Figure 6.7), which is indicative of a weak selection pressure on the distribution of defence compared to for instance, the selection pressure on the distribution of nitrogen. This spread reduces the relevance of a significant result on the mean values of the defence distribution. All reported differences were highly significant $(\mathrm{P}<0.001)$.

\section{Discussion}

\section{Biomass allocation patterns}

Our results on the optimal biomass allocation to leaves, stems and roots are consistent with the large body of literature on the optimal partitioning theory (OPT), which predicts that resource limitation increases biomass allocation towards plant parts that acquire that resource (Thornley, 1972; Bloom et al., 1985; Tilman, 1988). It has consistently been shown that biomass allocation towards roots increases with decreasing nitrogen availability and that biomass allocation to shoot, and especially stems, increases with decreasing light availability (Poorter et al., 2012). An extensive meta-analysis showed that light and nutrient availability are the predominant determinants of biomass allocation patterns, even after correcting for plant size (Poorter et al., 2012). These functional equilibria in biomass allocation patterns found in nature are an 


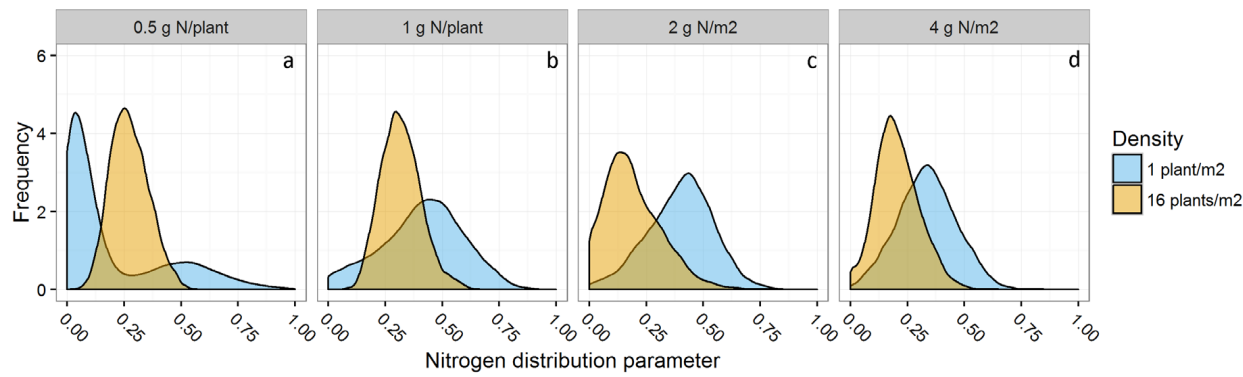

Figure 6.6 Frequency that a given nitrogen distribution (x-axis, distribution parameter values $<0.5$ represents a skew towards top leaves and values $>0.5$ the opposite) occurred in a plant populations that evolved for 75 generations in low $(1$ plant $\mathrm{m}-2)$ or high $(16$ plant $\mathrm{m}-2)$ plant densities with either a fixed soil nitrogen content per plant $(a, b)$ or a fixed soil nitrogen content per unit area $(\mathrm{c}, \mathrm{d})$.

emergent property of the evolutionary FSP model, resulting from the mechanisms of plant growth, competition for above- and belowground resources, and evolution.

\section{The effect of nitrogen availability and plant density on defence expression}

Our results show that the optimal level of defence increased with a decrease in nitrogen availability. This corresponds to proposed theories on the relation between low nutrient environments and plants that maintain a high level of defence due to the reduced capacity for re-growth (Cipollini et al., 2014). A meta-analysis showed that in high-nutrient environments an investment in plant defence has negative consequences for plant fitness while in low nutrient environments plant defence tends to have positive consequences for plant fitness (Koricheva, 2002). The results of the meta-analysis were consistent with both carbon and nitrogen based defences, which are predicted to be favoured in low (Tuomi et al., 1988; Herms \& Mattson, 1992) and high (Bryant et al., 1983) nutrient environments respectively. This consistency indicates that the shift in carbon-nitrogen balance associated with changing nitrogen availability is not responsible for the effect of nitrogen availability on the relation between defence and fitness (Koricheva, 2002). Little direct experimental evidence exists on the effect of nutrient availability on optimal defence levels. Our results show that an increase in plant density decreases the optimal level of defence expression if the nitrogen availability per plant is unaltered by the increase in plant density. This finding is in line with our current understanding of the linkage between plant density and plant defence through R:FR signalling, which mediates a decrease in plant defence expression at high plant densities (Ballaré, 2014; Ballaré \& Pierik, 2017). This plastic response allows plants to change their defence expression with the optimum 


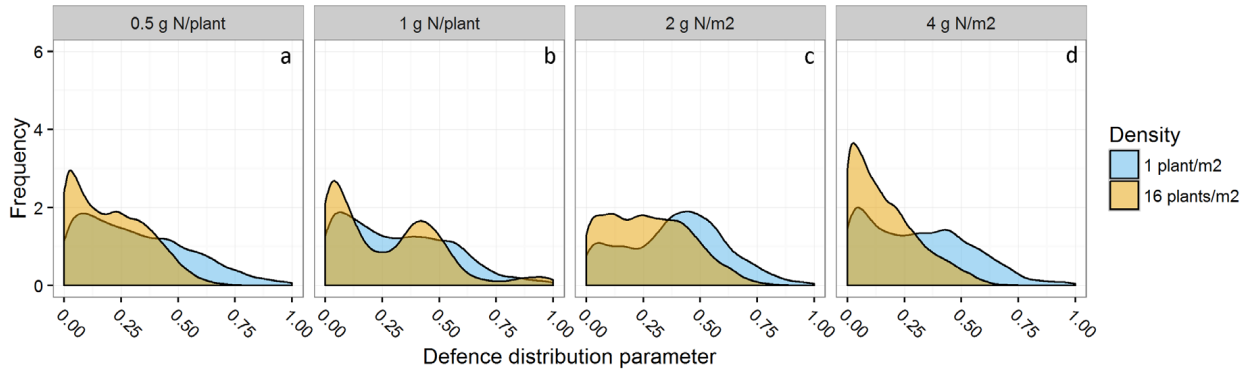

Figure 6.7 Frequency that a given defence distribution (x-axis, distribution parameter $<0.5$ represents a skew towards top leaves and values $>0.5$ the opposite) occurred in a plant populations that evolved for 75 generations in the presence of herbivore damage and in low (1 plant $\mathrm{m}-2)$ or high (16 plant $\mathrm{m}-2)$ plant densities with either a fixed soil nitrogen content per plant $(\mathrm{a}, \mathrm{b})$ or a fixed soil nitrogen content per unit area $(\mathrm{c}, \mathrm{d})$.

at any given plant density in an environment where the level of competition changes within and between generations. Research on the trade-off between investing in defence and investing in competitive ability has focussed on aboveground interactions rather than a combined effect of above- and belowground interactions. However, when considering that an increase in plant density also reduces the nitrogen availability per plant, our results show that the optimal level of defence increases with an increase in plant density. This suggests that light signal mediation of defence is beneficial to plant fitness under high nitrogen conditions, but not under low nitrogen conditions. We therefore expect low nitrogen environments to reduce the negative effect of R:FR on defence. While this effect of nitrogen on the R:FR mediation of defence has yet to be investigated experimentally, we do know of nitrogen affecting other R:FR responses. The impact of nitrogen on the R:FR mediation of tillering has been shown in rice (Zhong et al., 2002) and wheat (Sparkes et al., 2006), where low nitrogen availability increased the sensitivity of tiller death to R:FR. Belowground resources may thus play an important role in determining the adaptive value of plant defence in a competitive environment, but are often overshadowed by light effects in research on the effect of competition on optimal defence.

\section{Distribution of defence and nitrogen}

A second hypothesised benefit of the light mediation of plant defence is the optimisation of defence distribution in the canopy to protect the most valuable plant parts in terms of current resource allocation and future resource acquisition (Ballaré, 2014). In the model, the most valuable plant parts are the young leaves at the top of the canopy as they are most favourably positioned relative to the light gradient. This led the model to select for a distribution of nitrogen 
towards these young leaves in all treatment combinations, with a more non-uniform distribution in higher densities. This is in line with our understanding of the optimal distribution of nitrogen in the canopy, which is predicted and observed to favour plant parts that contribute most to resource acquisition (Hirose et al., 1987; Hirose, 2005; Hikosaka et al., 2016) and is more pronounced in dense canopies (Anten et al., 1995). The distribution of defence was expected to follow the distribution of nitrogen to optimise the defence of valuable young leaves and their nitrogen by reducing and redirecting herbivore damage. Our results show a weak selection on the distribution of defence in the canopy, slightly favouring plants that allocate defence towards young leaves. The direction of this selection is in line with our predictions based on optimal defence theory (McKey, 1974; Cipollini et al., 2014). However, the weak selection indicates that the benefits gained by optimising the distribution of defence in the canopy are much smaller than the benefits of optimising overall plant defence expression.

\section{Herbivore community dynamics and plant-herbivore interactions}

Our model assumes the herbivore damage on a plant to be independent from other plants, while herbivores are known to select their host based on defence expression (Schoonhoven et al., 2005; Viswanathan et al., 2005; Poelman et al., 2008b). Therefore, plant defence can be seen to function not only to reduce herbivore damage and to distribute it within the plant, but to deflect it to neighbouring plants. This function of defence makes the herbivore damage experienced by a plant dependent on the level of defence expression of its neighbours, and theoretically results in a tragedy of the commons where defence levels exceed their optimal value (McNickle \& Dybzinski, 2013). Accounting for the dynamics within a herbivore community and how it interacts with the plant community is a logical next step in the analysis of optimal defence (de Vries et al., 2017). However, this analysis becomes exceedingly challenging due to the complexity of herbivore communities and specificity of plant-insect interactions (Stam et al., 2014), the cross-talk between different systems of defence (Pieterse et $a l ., 2012$ ), and the occurrence of keystone herbivores in the insect community (Poelman \& Kessler, 2016).

\section{Plant architecture and plant plasticity}

To minimise the computational demand of the model we chose to simplify the plant architecture to the most basic shape that would retain the characteristics needed to simulate competition for above- and belowground resources. This basic plant architecture allowed us to study the general patterns in biomass allocation, defence expression and distribution of nitrogen and defence in 
the canopy. A model with a more complex representation of plant architecture is expected to retain the general patterns observed in this study, but would allow us to expand on the number of plant traits that make up a genotype in the model. This would make the plant genotype more complex, allowing for more interaction between plant traits, the emergence of more distinct strategies and potential co-existence of different genotypes or strategies in the simulated population. Many of the model simplifications are related to architectural traits that are known to be highly sensitive to changes in the light environment and nitrogen availability, such as branching (Domagalska \& Leyser, 2011) or leaf senescence (Lim et al., 2007). These morphological responses allow for better re-growth potential in low plant densities, potentially reducing the need to defend in favour of tolerating herbivore damage, especially in the absence of competition (McNickle \& Evans, 2018). In this study, we considered the optimal plant phenotype for a given set of environmental conditions that remain constant both within and over generations. However, plants growing under natural conditions face highly dynamic environmental conditions that change within and over generations. Plants have evolved plastic responses to express the multiple phenotypes in various environments, allowing plants to maximise their fitness over multiple generations (Bradshaw et al., 1965; Sultan, 2000). FSP modelling has shown that subtle variation in the sensitivity of a plastic response can have profound consequences for plant fitness (Bongers et al., 2018) and can therefore be a strong driver of evolution. Future development of our evolutionary FSP model can shed light on the optimal response of one or more plastic traits to an environmental condition that is set to vary within and between generations, such as plant density.

\section{Conclusions}

Here, we illustrated that the adaptive value of plant defence is dependent on the availability of, and competition for both above- and belowground resources. The value of investing in the acquisition or the protection of resources is a dynamic problem that is influenced by multiple ecological interactions, trade-offs and tragedies of the commons, and is therefore only relevant within an eco-evolutionary context. The model replicated the functional equilibria observed in nature as an emergent property that resulted from the mechanisms of plant growth, competition for above- and belowground resources, and evolution. This emergent nature makes the model a highly promising tool to simulate the evolution of plant-trait plasticity and community composition in dynamic and variable environments. 


\section{Supporting Information}

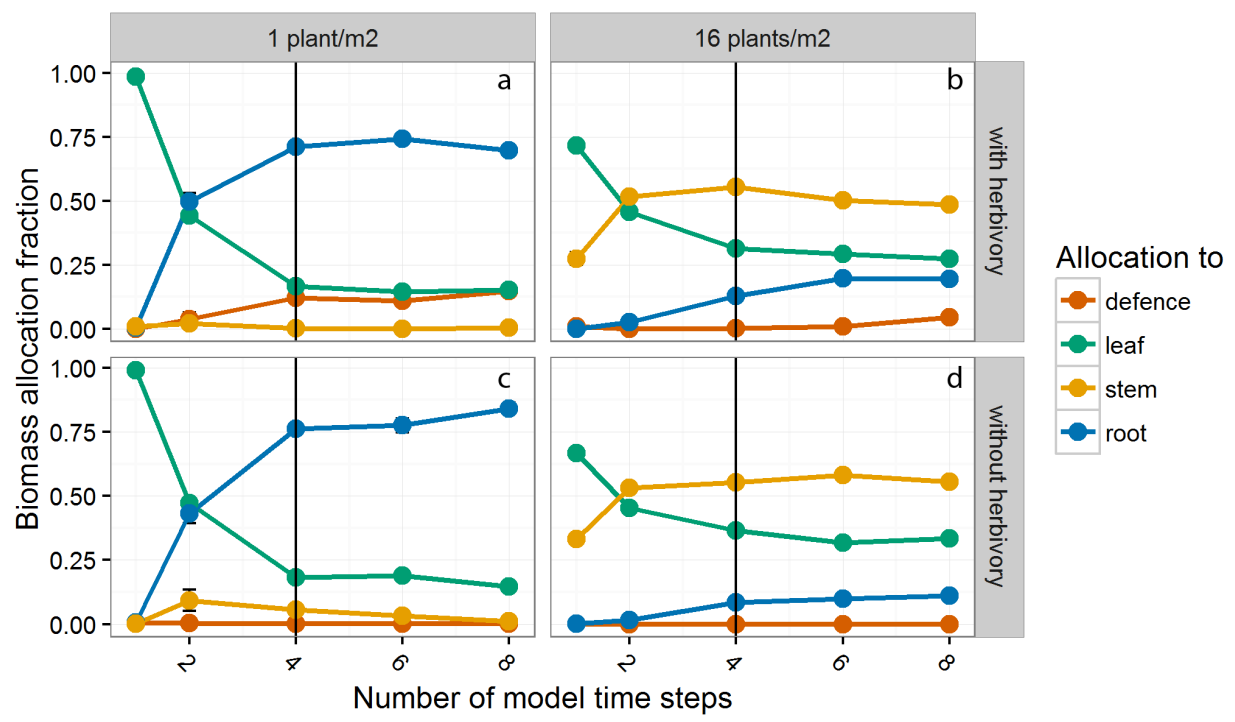

Figure S6.1. Sensitivity of biomass allocation patterns (y-axis) to the number of model time steps (x-axis) for plants in low (a,c; 1 plant $\mathrm{m}-2)$ and high (b,d; 16 plants $\mathrm{m}-2)$ plant density in the presence (a,b; $\mathrm{h}=0.1$ in eq. 6.15$)$ and absence (c, $\mathrm{d} ; \mathrm{h}=0$ in eq. 6.15$)$ of herbivory. A step size of four was chosen for the study, indicated by the vertical black line.
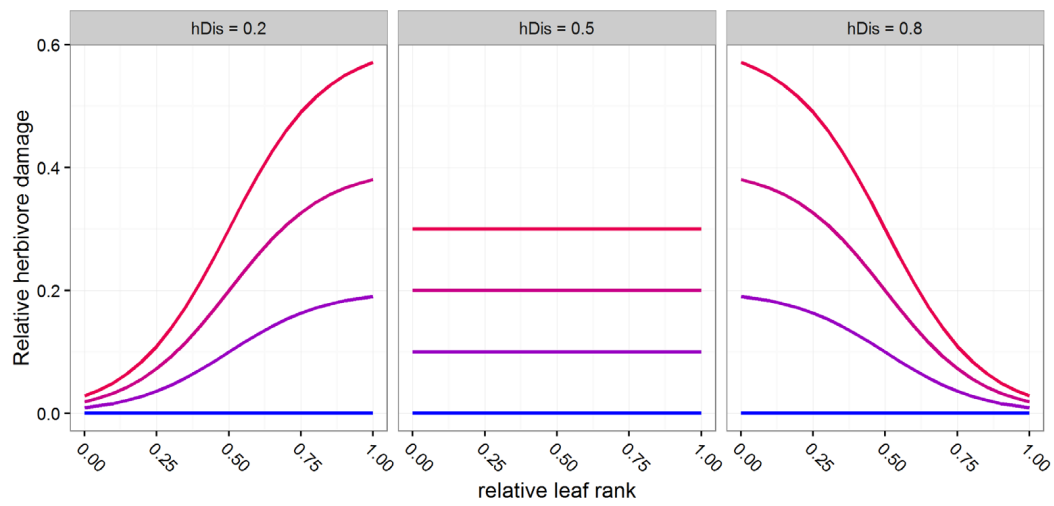

Herbivore damage coefficient

- $\mathrm{h}=0$

- $\mathrm{h}=0.1$

$-\mathrm{h}=0.2$

- $h=0.3$

Figure S6.2. Relative herbivore damage (y-axis) as a function of relative leaf rank (x-axis) for a range of herbivore damage coefficients ( $\mathrm{h}$ in eq. 6.15, low=blue, high=red) and herbivore distributions (hDis in eq 14,15 ) of $0.2,0.5$ and 0.8 , as described in eq. 6.15 . 


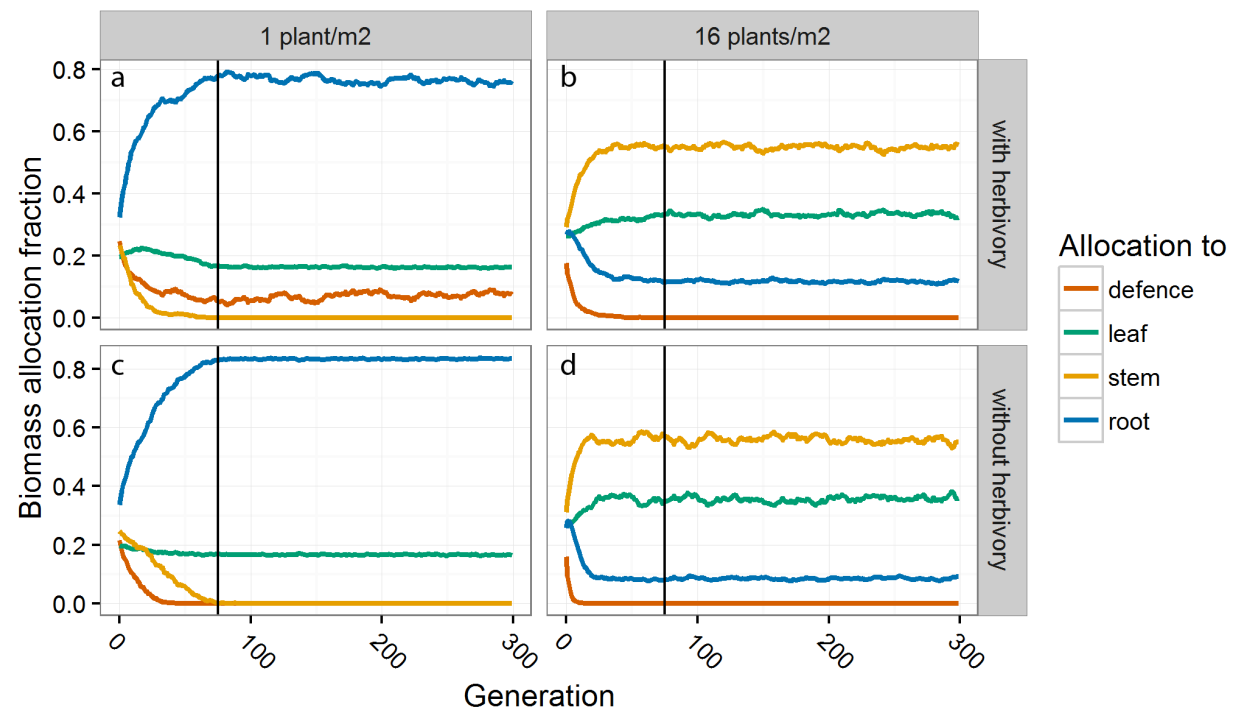

Figure S6.3. Changes in biomass allocation patterns (y-axis) over generations (x-axis) for plants in low (a,c; 1 plant m-2) and high (b.d; 16 plants $\mathrm{m}-2$ ) plant density in the presence (a,b; $\mathrm{h}=0.1$ in eq. 6.15$)$ and absence (c,d; $\mathrm{h}=0$ in eq. 6.15$)$ of herbivory. A model iteration length of 75 generations was chosen for the study, indicated by the vertical black line.

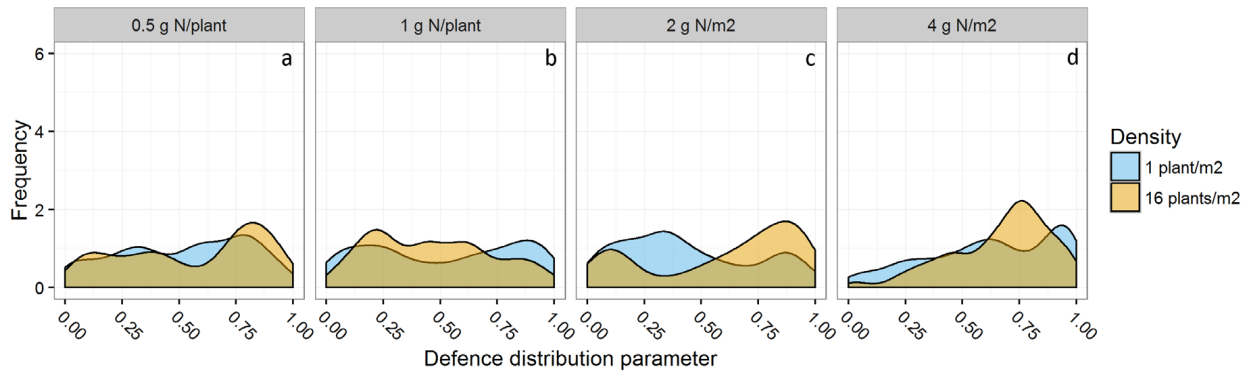

Figure S6.4. Frequency that a given defence distribution (x-axis, distribution parameter: values $<0.5$ represent a skew towards top leaves and values $>0.5$ the opposite) occurred in a plant populations that evolved in the absence of herbivore damage and in low $\left(1 \mathrm{plant} \mathrm{m}^{-2}\right)$ or high $\left(16\right.$ plant $\left.\mathrm{m}^{-2}\right)$ plant densities with either a fixed soil nitrogen content per plant $(\mathrm{a}, \mathrm{b})$ or a fixed soil nitrogen content per unit area $(\mathrm{c}, \mathrm{d})$. 


\title{
Chapter 7
}

\author{
General discussion
}




\section{Dynamic interactions shape growth-defence integration}

Plants grow in dynamic environments where they have to compete with other plants for resources and simultaneously protect themselves from heterotrophic organisms that feed on the plant. The optimal balance between the acquisition and protection of resources is dependent on ecological interactions within plant and insect herbivore communities, and is therefore highly dynamic in space and time (Heil, 2002; Strauss et al., 2002; Cipollini et al., 2014; Poelman \& Kessler, 2016; Ballaré \& Pierik, 2017; Züst \& Agrawal, 2017). It is largely unknown, however, how these dynamic ecological interactions drive natural selection, resulting in the physiological linkage between a neighbour proximity signal (i.e. the red to far-red ratio, R:FR) and direct defence expression commonly reported in plants (Moreno et al., 2009; Ballaré \& Pierik, 2017). In this thesis, I studied how localised damage by insect herbivores and competition between plants for limited resources such as light and nitrogen interact on ecological scales and drive natural selection on growth-defence integration in plants. In chapter 2, I made an argument for the importance of considering dynamic interactions that play out on ecological scales when studying the balance between competitive growth and defence. In subsequent chapters I utilised two functional structural plant (FSP) models that built on a mechanistic representation of plant physiology, ecological interactions and evolutionary dynamics. In chapter 3, I parameterised and validated a physiological FSP model of Brassica nigra that focussed on the mechanistic simulation of shade avoidance and defence responses and analysed the impact of herbivore distributions within the plant on plant fitness. In chapter 4, I used the same model to analyse the costs and benefits of plant defence in an ecological setting where plants competed with their neighbours for light and faced different levels of herbivory. In chapter 5, I analysed the function of R:FR mediation of defence in a dynamic environment of competition for light and herbivory and placed this physiological response into an evolutionary perspective. Finally, in chapter 6 , I utilised an evolutionary model of a generic dicot species that mechanistically simulates ecological and evolutionary dynamics to analyse how ecological interactions between plants drive natural selection on growth-defence integration. The FSP models that I used in this thesis, explicitly consider the 3D plant architecture and its interaction with the light environment to mechanistically simulate plant growth and development, from which the interaction between plants and its effect on plant fitness naturally emerge. The mechanistic nature of these models makes them great tools for quantifying both the direct effect of plant defence investment on plant fitness and how this plays out on ecological and evolutionary scales through interactions with other plants. 
In this general discussion, I will expand upon the conclusions and concepts discussed in this thesis by proposing additional ideas and providing some future perspectives in the fields of plant-insect ecology and FSP modelling. I will focus on current challenges and future perspectives of studying dynamic systems on eco-evolutionary scales, and highlight the role of FSP modelling in studying these dynamic systems. Furthermore, I will provide additional simulations that illustrate the concepts discussed here and end with concluding remarks.

\section{Using FSP models to simulate dynamic environments: challenges and opportunities}

When using a modelling approach to study how ecological interactions shape the integration of competitive growth and defence, mechanistic simulation of light competition is crucial. Both the physiological and evolutionary FSP models used in this thesis simulated the feedback from plant architecture to light interception, which in turn affected plant architecture through plant growth. Additionally, the physiological FSP model simulated the feedback between the light environment and plant architecture through shade avoidance responses to changes in the R:FR ratio (Ballaré \& Pierik, 2017) and simulated plant growth through organ level source-sink dynamics (Evers \& Bastiaans, 2016). The evolutionary FSP model forwent these mechanisms in favour of computational efficiency by implementing a smaller temporal resolution combined with a more descriptive representation of plant architecture. Plant growth was simulated through light interception and photosynthesis, but was driven by genotype dependent biomass allocation patterns rather than source-sink dynamics. These methodological changes allowed the evolutionary FSP model to simulate a plant population over generations and focus on the evolutionary dynamics of plant growth-defence integration. This evolutionary perspective is invaluable in understanding how ecological interactions in a dynamic environment impact natural selection, which is proven to be extremely difficult and time consuming with a physiological FSP model (see chapter 5 of this thesis).

One of the main challenges of physiological FSP models is placing their findings in an ecological or evolutionary context, as it is almost impossible to quantify the eco-evolutionary significance of different treatment combinations comparative to each other. Therefore, we require an evolutionary modelling approach to assess how the eco-evolutionary significance of a given set of environmental conditions depends on the frequency in which these conditions occur, and the selection pressure imposed by that environment. In environments were plants compete for limited resources, plants tend to overexpress traits related to resource acquisition compared to the population-level optimum to maximise individual performance, termed a 
tragedy of the commons (TOC) (Falster \& Westoby, 2003; McNickle \& Dybzinski, 2013). This overexpression of traits related to resource allocation may go at the expense of other functions such as defence (Züst \& Agrawal, 2017). The presence of these TOC's and trade-offs between plant traits can obscure what is the optimal strategy in a given environment, which is likely not static over time, but rather highly dynamic within and between generations. Therefore, a given phenotype observed in a natural system is not necessarily optimal for the environment in which it is observed, but rather adapted to a dynamic environment with variable selection pressures that might not be apparent at all times. This can certainly be the case for plant defence expression as herbivore abundance varies greatly from one year to the next, creating a varying landscape of selection pressures (Van Zandt \& Agrawal, 2004). Additionally, the level of competition experienced by $B$. nigra can vary greatly between growing seasons as it tends to grow in environments ranging from barren soil to dense monospecific stands and diverse grasslands with hetero-specific competitors (Lankau \& Strauss, 2008). How a dynamic environment changes the selection pressure experienced by a plant may be exemplified using an adaptation of the evolutionary model used in chapter 6 by changing plant density to vary between generations (see Box 7.1). The additional simulations illustrated in Box 7.1 show that a dynamic plant density selected for a high density phenotype, whereas a constant plant density selected for a low density phenotype (Figures 7.1 and 7.2). Plants are known to exhibit plastic responses to environmental signals to maximise their fitness in dynamic environments (Sultan, 2000). However, a plastic response will not necessarily allow the plant to express the optimal phenotype in all possible environments (see chapter 5, Bongers (2017)). This illustrates that plants fit on a scale between two extreme strategies, a jack-of-all trades that is able to maintain fitness in unfavourable environments, versus a master-of-some that is able to maximise fitness in favourable environments (Richards et al., 2006). These strategies exemplify once more that we must be cautious in assuming that a plant is expressing the optimal phenotype for the environment it is currently observed in, but rather consider how the environment changes within and between generations. This evolutionary FSP model is an excellent tool to assess the ecoevolutionary significance of a plant phenotype in a variable and dynamic environment. However, the model currently simulates a static rather than a plastic plant phenotype, which is important to consider in a variable environment. A key challenge in advancing evolutionary FSP models to simulate variable environments is the implementation of plant plasticity, as it requires a higher temporal resolution, while still maintaining the computational efficiency needed to simulate selection over generations. I expect future evolutionary FSP models to 


\section{Box 7.1 The effect of variable plant density on optimal stem allocation}

The model presented in chapter 6 was used to run additional simulations to show the effect of a variable plant density and variable plant distance on the optimal allocation of biomass to stems. As reference (Fixed density, Fixed distance) 100 plants were simulated in a field of $5 \times 5 \mathrm{~m}$ with a plant distance of $0.5 \mathrm{~m}$, resulting in a plant density of 4 plants $\mathrm{m}^{-2}$. Two other scenarios were simulated either with varying distance between plants (Fixed density, Variable distance, Figure 7.1) or with varying the size of the field, and hence, the plant density (Variable density, Fixed distance), at the start of a new generation. The average density over generations was equal in all of these scenarios, and the plants experienced intermediate levels of herbivore damage $(0.15)$ and nitrogen availability $\left(0.5 \mathrm{~g} \mathrm{~N}\right.$ plant $\left.^{-1}\right)$. The simulated plants in the variable density and distance treatments evolved a higher density phenotype, indicated by an increased investment in stems, compared to plants simulated in the reference scenario with a fixed density and distance (Figure 7.2).

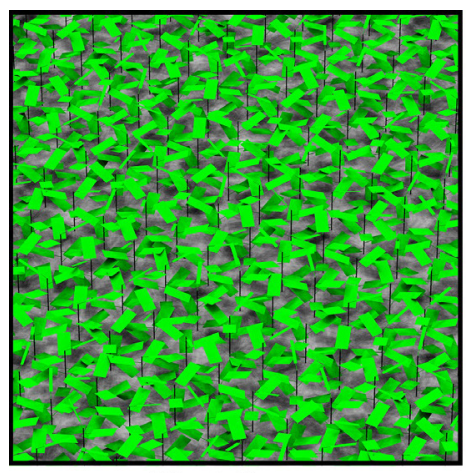

Fixed distance

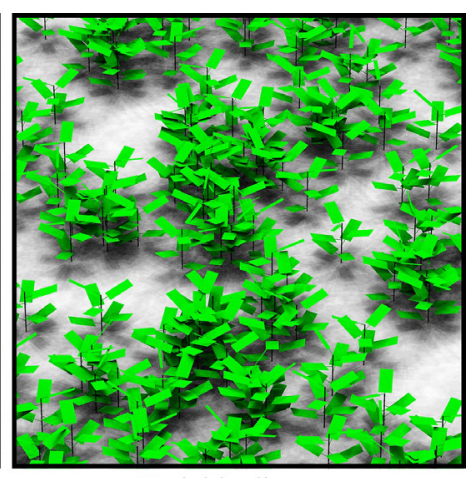

Variable distance

Figure 7.1 Two plant populations of equal plant density with either a fixed (a) or variable (b) distance between plants. The plant population consists of a single phenotype for visualisation purposes and is not representative for the plant populations of the simulations.

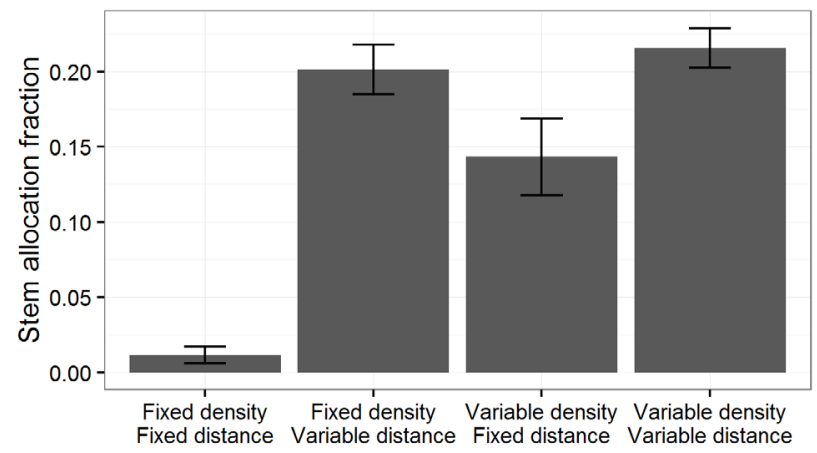

Figure 7.2 The average fraction of biomass allocated to stems (y-axis) of a population that evolved for 75 generations in a plot where the density and distance were either fixed or varied (x-axis). The reference scenario simulated a density of 4 plants $\mathrm{m}^{-2}$ at a plant distance of $0.5 \mathrm{~m}$. 
overcome the resolution-efficiency trade-off and evolve to a methodology that is able to scale from mechanistic representations of plant physiological to eco-evolutionary dynamics. The main strength of this approach lies in how the optimal phenotype for a given environment is an emergent property that arises not from a descriptive set of assumptions, but from a set of mechanisms that act on physiological, ecological and evolutionary scales.

\section{Tolerance versus defence: the importance of simulating plant form and function}

An alternative strategy to the production of costly secondary metabolites to defend against herbivory is the ability to regrow damaged tissues after herbivore attack, allowing plants to tolerate rather than prevent the damage done. Plants are able to increase tolerance to herbivore damage through traits that increase photosynthetic capacity, increase branching or tillering, change carbon allocation patterns after damage occurred, or promote the availability of preemptive storage (Rosenthal \& Kotanen, 1994; Strauss \& Agrawal, 1999; Stowe et al., 2000; Krimmel \& Pearse, 2016). All of these traits are severely hindered by competition with neighbouring plants because of a reduction in light availability, an inhibition of the plant's ability to branch and tiller, and stronger competition for belowground resources. Therefore, tolerance is a viable strategy in environments where competition pressure is low (McNickle \& Evans, 2018), whereas plants will have to rely on their ability to defend against herbivore damage in more competitive environments. Plants growing in low competition environments are inherently more tolerant to herbivore damage, due to the absence of non-self-shading. In these environments, the light capture lost through leaf area removal by herbivore damage may be compensated by other leaves of the same plant (Trumble et al., 1993). In more competitive environments, where plants experience higher levels of non-self-shading, the light capture lost through leaf area removal by herbivores may instead be captured by neighbouring plants, provided these have not been similarly damaged. Furthermore, tolerance and competitive ability may be negatively correlated as the allocation of pre-emptive storage to roots, which makes a plant more tolerant to herbivore damage but may inhibit the plant's ability to compete for light or other resources (Hochwender et al., 2000; Stowe et al., 2000). However, it is unclear if a trade-off between tolerance and resistance to herbivory exists, as evidence has been found to both support (Fineblum \& Rausher, 1995; Stowe, 1998) and refute (Fry, 1992; Rosenthal \& Kotanen, 1994) this hypothesis. Contrary to plant defence responses that are actively induced by herbivore feeding, tolerance to herbivore damage has been linked to intrinsic mechanisms rather than active responses (Strauss \& Agrawal, 1999; Cipollini, 2007). This suggests that a plant's ability to tolerate herbivore damage mostly emerges passively from mechanisms of light 
capture, source-sink relations and branching dynamics, rather than being an active response to herbivore damage beyond these indirect effects. While this implies no direct physiological linkage (e.g. an active response) between herbivory and a plants ability for regrowth, the two are linked from an evolutionary perspective. This can lead to strategies that makes plants more tolerant to herbivore damage through intrinsic differences in biomass allocation patterns or shade avoidance responses, without relying on active responses to herbivore damage. Native genotypes of Jacobaea vulgaris that experience a herbivore community dominated by specialists exhibited a greater capacity for regrowth than invasive genotypes experiencing a herbivore community dominated by generalists (Lin et al., 2015). This exemplifies how herbivory may act as a driver of selection on the expression of growth and shade-avoidance mechanisms, especially when providing a cheaper or more effective form of resistance against herbivore damage than secondary metabolites. This not only signifies the importance of including shade avoidance responses in models of defence against herbivory, but also the importance of considering shade-avoidance responses in the context of herbivory. Physiological FSP models are excellent tools to study the tolerance-resistance trade-off as they mechanistically simulate source-sink dynamics, plant morphology, and shade avoidance responses that drive the interactions between resource competition, tolerance, and resistance to herbivory.

The two FSP models used in this thesis exemplify the importance of mechanistically simulating plant form and function to allow analysis of tolerance to herbivore damage as an emergent property. The physiological model of $B$. nigra used in chapters 3-5 simulated the mechanisms of source-sink dynamics and branching that would allow tolerance to be an emergent property of the model. Conversely, the evolutionary model used in chapter 6 used a far more static representation of plant morphology lacking the mechanisms to allow tolerance to be an emergent property of the model. The differences in how the two models simulate the mechanisms that promote tolerance to herbivory is reflected in the relation between optimal defence allocation and plant density reported in chapters 5 and 6 . On the one hand, the physiological model predicted optimal defence levels to be highest in intermediate densities. This can be caused by plasticity in shade-avoidance responses that allows the plant to tolerate herbivore damage in low densities and impose the trade-off between competitive growth and defence in high densities. The evolutionary model on the other hand, predicted optimal defence levels to be higher in low densities due to the static representation of plant morphology (e.g. lack of plastic shade-avoidance responses), restricting the plant's ability for regrowth. These 


\section{Box 7.2 The effect of variable herbivore distribution on optimal defence expression}

The model presented in chapter 6 was used to run additional simulations to show the effect of variable herbivore pressure on biomass allocation patterns. 100 plants were simulated in a field of $5 \times 5 \mathrm{~m}$ with a plant distance of $0.5 \mathrm{~m}$, resulting in a plant density of 4 plants $\mathrm{m}^{-2}$. The plants in all of these scenario's experienced intermediate levels of herbivore damage $(0.15)$ and nitrogen availability $\left(0.5 \mathrm{~g} \mathrm{~N}\right.$ plant $\left.^{-1}\right)$. Plants were infested with either control herbivores that evenly distributed over the canopy, generalist herbivores that avoided high defence leaves or specialist herbivores that preferred high defence leaves. In contrast to the model used in chapter 6 , where herbivore pressure was fixed at the plant level, the herbivore pressure in these simulations was fixed at the plot level and could vary between plants. The herbivore pressure experienced by a plant was, therefore, dependent on the defence expression of its own leaves relative to that of all other plants in the simulated plot. This resulted in a TOC where generalist herbivores selected for a higher level of defence and specialist herbivores selected for a lower level of defence compared to the control herbivores.

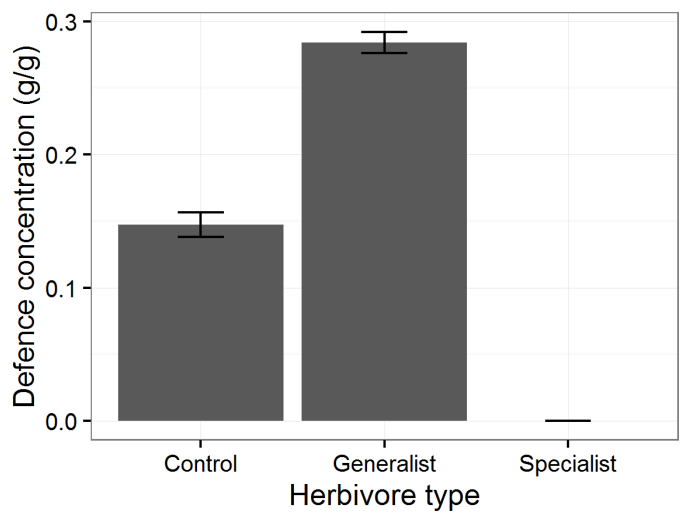

Figure 7.3 Average defence concentration ( $g$ defence $\mathrm{g}^{-1}$ leaf, $\mathrm{y}$-axis) of plants after 75 generations of evolution in a plot where plants were infested with either control herbivores that evenly distributed over the canopy, generalist herbivores that avoided high defence leaves or specialist herbivores that preferred high defence leaves (x-axis). In these simulations the herbivore pressure was fixed at the plot level and could vary between plants, in contrast to the model used in chapter 6 where herbivore pressure was fixed at the plant level.

differences highlight the importance of mechanistic rather than descriptive simulation of plant form and function when studying plant resistance to herbivory in an eco-evolutionary context.

\section{Plant-insect interactions and FPS modelling}

Taxon-specific secondary metabolites used in plant defence (e.g. qualitative defence) are used by insect herbivores in host selection, repelling generalist herbivores and attracting specialist herbivores for which it provides a reliable host selection cue (Jaenike, 1990; Badenes-Perez et 
al., 2014; Wei et al., 2015). These host-plant selection preferences make the herbivore pressure experienced by a plant dependent on the composition of the insect community, its own defence expression, as well as that of its neighbours (Bustos-Segura et al., 2017). Thus, the goal of plant defence may be to both minimise the damage of an attacking herbivore, and to deflect that herbivore onto a neighbouring plant, proving both direct and indirect benefits to the plant. This theoretically results in a TOC that drives the expression of defence provided by taxon-specific secondary metabolites away from what is optimal on the population level, in an attempt to deflect herbivore pressure onto neighbouring plants (McNickle \& Dybzinski, 2013). The direction in which this TOC drives selection for plant defence depends on the composition of the insect community, where generalists select for, and specialists select against, the expression of qualitative defence (Lankau, 2007). The consequences of this variable selection pressure may be tested using an adaptation of the evolutionary model used in chapter 6 , where herbivore damage on a leaf is dependent on the level of herbivore specialisation and the leaf defence expression relative to that of all other leaves in the plot (see Box 7.2). The additional simulations presented in Box 7.2 confirm that the preferences of generalists and specialists lead to opposite selection pressures and a TOC in defence expression. The results show that generalist herbivores that forgo leaves with high levels of secondary metabolites select for overexpression of plant defence relative to a control herbivore that evenly distributes itself over the canopy (Figure 7.3). Conversely, specialist herbivores that prefer leaves with high levels of secondary metabolites drive selection away from plant defence expression, in this case resulting in a phenotype that completely forwent investing in secondary metabolites (Figure 7.3). This selection against the investment in taxon-specific secondary metabolites may lead to an increased investment in quantitative forms of defence, such as trichomes, leaf toughness or tannins.

Plant-herbivore interactions are often studied in isolation, whereas in natural systems these interactions always occur in the context of a multitude of other interactions that influence how a single plant-herbivore interaction plays out (Strauss \& Irwin, 2004; Stam et al., 2014; Kroes et al., 2016; Stam et al., 2017). While it is still relevant to study these isolated interactions to understand the mechanisms involved in the interaction, their relevance diminishes when placed in an ecological or evolutionary context. However, studying plant-herbivore interactions in the context of its natural setting is exceedingly difficult to control and requires large scale experiments and the use of complex statistical tools. While this would provide opportunities for modelling approaches, I feel FSP modelling is unsuitable to study interactions between plant 
and herbivore communities because the species-specific nature of individual plant-herbivore interactions (Van Zandt \& Agrawal, 2004; Poelman et al., 2008). Interactions between members of the herbivore community are driven by their induction of plant defence, which may persist over the entire growing season and thereby influence subsequent colonisation of the plant by other herbivores (Stam et al., 2014). These plant-mediated interactions between the members of the herbivore community are specific to the plant and herbivore species and thereby forego the emergent nature of FSP modelling that defines the approach: The strength of FSP modelling lies in its ability to simulate interactions based on the mechanisms that underlie the interaction rather than using descriptive interaction coefficients, which are prevalent in mathematical and statistical models. For example, FSP modelling may be a strong tool to study how herbivore feeding preferences or plant performance are driven by the herbivore's feeding guild and level of specialisation. This mechanistic approach makes the outcome of a plantherbivore interaction or a plant-mediated interaction between two herbivores an emergent property, rather than an input parameter of the FSP model. However, this approach is unlikely to provide a realistic representation of a given plant-herbivore interaction, as both the herbivore and the plant responses are specific to both the plant and herbivore species involved. It has been shown that a specific plant-herbivore interaction not always conforms to, and sometimes even contradicts expectations based on generalisations such as host specialisation or feeding guild (Stotz et al., 2002; Schmidt et al., 2005; Weech et al., 2008; Bruessow et al., 2010; Ballaré, 2011). The outcome of a given plant-herbivore interaction is determined by a complex system of plant phytohormones (Spoel \& Dong, 2008; Ballaré, 2011; Thaler et al., 2012), which are activated by effectors, herbivore- and damage-associated molecular patterns (HAMP's and DAMP's respectively) related to the herbivore and its life history (Musser et al., 2002; Felton \& Tumlinson, 2008; Heidel-Fischer et al., 2014; Acevedo et al., 2015; Stuart, 2015). The mechanisms that underlie the outcome of a given plant-herbivore interaction are therefore too detailed and complex to realistically implement into an FSP model. This problem can be addressed by either describing a given plant-herbivore interaction using an interaction coefficient, or to simulate mechanisms of a higher level of integration and forgo relating the outcome of the model to a specific plant-herbivore interaction. These two ways of simplifying plant-herbivore interactions in FSP models are discussed in more detail later in this chapter. The realisations that plant-insect interactions are highly species-specific and that competition for resources on ecological and evolutionary scales is far more complex than initially anticipated, have led to a shift in perspective from plant-herbivore to plant-plant interactions. 
In chapter 2 of this thesis, I made an argument for the inclusion of dynamic interactions that play out on ecological and evolutionary scales when considering growth-defence integration. In this argument, I focussed on the interactions between the plant and herbivore communities as an important driver of the trade-off between competitive growth and defence expression. Even though this thesis has shown that it is paramount to consider the balance between competitive growth and defence in an eco-evolutionary context, the focus has shifted from plant-insect interactions to the interactions between plants, driven by competition for resources and by herbivory.

\section{Balancing the acquisition and protection of multiple resources}

Plants require multiple resources to grow, which are acquired both above- (light) and belowground (water, nutrients). Internally, plants have to balance the acquisition of these resources as their growth is generally limited not by total resource availability but by the availability of the scarcest resource. This balancing can be observed in the ratio of biomass allocated to roots and shoots, which responds to resource limitation by increasing biomass partitioning to the plant parts responsible for the acquisition of that resource (Ågren \& Ingestad, 1987; Poorter et al., 2012; Ledo et al., 2018). While plants show strong phenotypic responses to impending competition for light, biomass partitioning is more responsive to limitations in belowground resources (Poorter \& Nagel, 2000). This suggests that belowground resource limitation would be just as, if not more, liable to resource allocation trade-offs than aboveground resource limitation. However, research on the trade-off between competitive growth and defence has focussed on shade-avoidance growth to maximise light capture (Züst \& Agrawal, 2017) and has largely disregarded belowground resource acquisition. However, belowground resources, especially nutrients, can be of great importance in the context of growth-defence trade-offs, as herbivores tend to prefer feeding on plant parts with high concentrations of nutrients (Cates, 1980; Schoonhoven et al., 2005). Nutrients such as nitrogen are mostly allocated to green leaves during the vegetative phase (Rossato et al., 2001), favouring young and high light leaves to maximise photosynthesis (Anten et al., 1995; Hirose, 2005; Hikosaka et al., 2016). These leaves contribute most to light capture and are, therefore, vital to plant competitiveness (see chapter 3 ). During the generative phase, nitrogen allocation patterns shift to generative plant parts, remobilising nitrogen that was previously allocated to leaves, stems and roots (Rossato et al., 2001). The loss of high nutrient plant parts to herbivore attack not only robs the plant of the carbon used to build these tissues and the future photosynthetic gain and fitness they represent (see chapter 3 of this thesis), but also of the 
limited nutrients they contain. It is therefore vital to plant fitness to protect plant parts with high concentrations of nutrients, especially when these nutrients are limiting plant growth as their loss has a disproportionate effect on plant fitness (see chapter 6). Future experimental research can test these predictions made by the FSP models and elucidate the effect of nutrient limitation on how plants manage their investments in competitive growth and defence.

\section{Future perspectives for FSP models of growth and defence}

As with any modelling approach, the FSP models used in this thesis contain assumptions to simplify the study system and make predictions in the context of the mechanisms included, explicitly or implicitly, as well as those excluded for simplicity's sake. In the physiological model used in chapters 3-6, I focussed on a single plant species, B. nigra, growing in monostands with different planting densities. Current FSP models are well equipped to simulate the mono-stands that characterise some natural and most agricultural systems, and may be utilised to design idiotypes, and help optimise architectural and physiological traits of crop plants (Lynch, 2013; Perez et al., 2018). As discussed in chapter 5, physiological FSP models may play a role in elucidating whether decoupling the physiological linkage between shade avoidance growth and defence in crops would increase crop performance by increasing herbivore resistance and decreasing pesticide use (Ballaré \& Pierik, 2017). In natural ecosystems however, plants are often part of diverse plant communities rather than monostands, though natural mono-stands also exist. FSP models that simulate multiple plant species have been developed to study intercropping (Postma \& Lynch, 2012; Barillot et al., 2014; Zhu et al., 2015), but have only recently expanded to the simulation of multi-species interactions in natural systems (Faverjon et al., 2018). In chapter 6, I utilised an evolutionary FSP model that simulates how a diverse community of plants evolves over time. An evolutionary FSP model such as the one used in chapter 6 is particularly well equipped to simulate natural systems and the ecological and evolutionary dynamics that shape plant communities. Future advancements in this modelling approach may open avenues to study how natural selection may lead to coexistence of different plant species and the formation of biodiversity in plant communities.

In this thesis, I focussed on competition between plants for a single resource (light, chapters 3-6), or a pair of resources (light and nitrogen, chapter 7). While FSP models of aboveground (Evers et al., 2007; Lopez et al., 2010; Vos et al., 2010; Evers et al., 2018) and belowground (Diggle, 1988; Pagès et al., 1989; Lynch et al., 1997; Dunbabin et al., 2013) plant architecture and resource acquisition have existed for decades, models that cover both above- 
and belowground resources have been only recently developed (Louarn \& Faverjon, 2018). The development of fully mechanistic representations of both above- and belowground plant architecture is challenging from both a physiological and a computational perspective as the level of spatial and temporal detail differs between above- and belowground processes. Models of root architecture require a high spatial resolution, going to the level of fine roots or even root hairs, and require a high temporal resolution to simulate fluxes of water and nutrients. This combination makes mechanistic root architectural models computationally expensive, requiring approximation approaches to scale the details of root architecture and uptake dynamics to match the larger spatial and temporal scales of aboveground plant models. Future FSP models that merge above- and belowground architectures, processes, resources, and ecological interactions may play a pivotal role in our understanding of plant ecology and evolution in a dynamic environment.

The FSP models presented in this thesis applied a generic representation of herbivory, disregarding any interactions between different species in the herbivore community. In FSP models, herbivory can be simulated as either individual herbivores using agent-based modelling techniques (see chapter 3), or as a mathematical function that describes the average damage done by a community of herbivores (see chapters 4-6). The agent-based modelling approach is more mechanistic in nature and requires input on the growth, development, and behaviour of an individual herbivore and can be parameterised for different herbivore species using experimental data. The advantage of using an agent-based approach is the ability to mechanistically simulate a herbivore community and its effects on a stand of plants. Conversely, the mathematical representation of a herbivore community is more descriptive and uses only a few parameters that describe mechanisms such as herbivore arrival, abundance, growth rates, etc. aggregated into one function rather than separately defined. Another advantage of this mathematical representation of a herbivore community compared to the agentbased approach is the ability to reduce stochasticity, which greatly reduces the number of simulations required to show an effect. Research focussing on plant community dynamics benefits from the simplicity of the mathematical approach, whereas research on dynamic interactions between the plant and herbivore communities benefits from the mechanistic nature of the agent-based approach. One of the challenges in the field of plant-insect ecology is elucidating how interactions between herbivores shape the herbivore community experienced by a plant and how this herbivore community affects plant fitness (Stam et al., 2014; Poelman \& Kessler, 2016). To this end, an FSP model can be developed that couples plant architecture 
and an agent-based representation of herbivory, coupled with an interaction matrix. This matrix describes the effect of one herbivore species (inducer) on the colonisation and performance of all other herbivore species (receiver) in the community through its induction of the plant's defences. This interaction matrix, combined with an agent-based representation of individual herbivores, may be used to study the effect of a given herbivore community composition or interaction matrix on crop performance, plant fitness, or optimal defence expression. While the development, parameterisation and validation of such a model is challenging, it would provide opportunities for research that would be exceedingly challenging to conduct solely through experiment approaches.

In this thesis, I focussed on a single neighbour proximity signal that mediated both shadeavoidance and defence, i.e. the R:FR ratio, in the context of source-sink driven plant growth. However, other light cues such as blue (Keuskamp et al., 2012), green (Zhang et al., 2011) and UV (Ulm \& Jenkins, 2015), volatile cues such as ethylene (Ecker \& Davis, 1987; Pierik et al., 2004a; Pierik et al., 2004b), and mechanical cues such as touch (de Wit et al., 2012), all play a part in the expression of the shade avoidance syndrome (Pierik et al., 2013; Ballaré \& Pierik, 2017). A low R:FR ratio is known to downregulate direct forms of defence (Cortes et al., 2016; Ballaré \& Pierik, 2017), indirect forms of defence (Izaguirre et al., 2013) and volatile emissions (Cortes et al., 2016), although the latter actually increased indirect defence through the attraction of predators. Additionally, UV-B (Mazza \& Ballaré, 2015), ethylene (Adie et al., 2007), as well as other herbivore-induced volatile cues (Bruin \& Dicke, 2001; Kessler et al., 2006) are known to play a role in the control of plant defence. However, the relevance of other neighbour proximity signals in plant defence integration is still unknown. These cues and their effect on plant defence expression are potentially linked to plant ontogeny, which is known to affect both shade avoidance responses (Weinig, 2007) and whole-plant defence expression (Boege \& Marquis, 2005; Barton \& Koricheva, 2010; Barton \& Boege, 2017). A major challenge for future experimental research is elucidating what neighbour detection signals play a role in mediating plant defence, how these signals are integrated in the mediation of plant defence, and how this integration is linked to plant ontogeny. Then, future FSP models can help elucidate the role of these neighbour detection signals relative to one another and relative to the stage in plant development in which the signal occurs. This is exemplified by the use of FSP modelling to elucidate the timing of touching leaf tips and R:FR signals in the regulation of a hyponastic response in Arabidopsis thaliana (de Wit et al., 2012). Additionally, FSP models can place the responses to these signals in an ecological context and elucidate their evolutionary 
significance.

\section{Concluding remarks}

Research on plants and insects covers a wide range of temporal and spatial scales, from molecular processes that take seconds to evolutionary processes that happen on landscape scales and take thousands of years. Integrating multiple temporal and spatial scales is imperative to a deep understanding of plant functioning in both natural and agricultural settings. In my research, I utilised a 3D modelling tool that simulated how physiological mechanisms translate to ecological and evolutionary scales. With this tool, I have shown that the integration of competitive growth and defence in plants is shaped by ecological interactions in dynamic environments, which is apparent from physiological to evolutionary scales. FSP models have been developed for decades and the methodology has matured to become a potent tool in studying the interactions between a plant's internal processes and its environment. Further advancements of the methodology will be driven by the development of novel simulation techniques, innovations in computer sciences and progression of our understanding of plants and their environment through both experimental and modelling work. In time, I expect FSP modelling to become imperative in guiding future experimental research, plant breeding and crop management. 



\section{References}

Acevedo FE, Rivera-Vega LJ, Chung SH, Ray S, Felton GW. 2015. Cues from chewing insects-the intersection of DAMPs, HAMPs, MAMPs and effectors. Current Opinion in Plant Biology 26: 80-86.

Adie B, Chico JM, Rubio-Somoza I, Solano R. 2007. Modulation of plant defenses by ethylene. Journal of Plant Growth Regulation 26(2): 160-177.

Agrawal AA. 2000. Benefits and costs of induced plant defense for Lepidium virginicum (Brassicaceae). Ecology 81(7): 1804-1813.

Agrawal AA, Lau JA, Hamback PA. 2006. Community heterogeneity and the evolution of interactions between plants and insect herbivores. The Quarterly Review of Biology 81(4): 349-376.

Ågren GI, Ingestad T. 1987. Root: shoot ratio as a balance between nitrogen productivity and photosynthesis. Plant, Cell \& Environment 10(7): 579-586.

Alba C, Bowers MD, Hufbauer R. 2012. Combining optimal defense theory and the evolutionary dilemma model to refine predictions regarding plant invasion. Ecology 93(8): 1912-1921.

Anderson KE, Inouye BD, Underwood N. 2015. Can inducible resistance in plants cause herbivore aggregations? Spatial patterns in an inducible plant/herbivore model. Ecology 96(10): 2758-2770.

Anten NP, Martínez-Ramos M, Ackerly DD. 2003. Defoliation and growth in an understory palm: quantifying the contributions of compensatory responses. Ecology 84(11): 2905-2918.

Anten NPR. 2002. Evolutionarily stable leaf area production in plant populations. Journal of Theoretical Biology 217(1): 15-32.

Anten NPR, Pierik R. 2010. Moving resources away from the herbivore: regulation and adaptive significance. New Phytologist 188(3): 643-645.

Anten NPR, Schieving F, Werger MJA. 1995. Patterns of light and nitrogen distribution in relation to whole canopy carbon gain in C-3 and C-4 monocotyledonous and dicotyledonous species. Oecologia 101(4): 504-513.

Anten NPR, Vermeulen PJ. 2016. Tragedies and Crops: Understanding Natural Selection To Improve Cropping Systems. Trends in Ecolology \& Evolution 31(6): 429-439.

Aphalo PJ, Ballaré CL, Scopel AL. 1999. Plant-plant signalling, the shade-avoidance response and competition. Journal of Experimental Botany 50(340): 1629-1634.

Augner M, Fagerstrom T, Tuomi J. 1991. Competition, defense and games between plants. Behavioral Ecology and Sociobiology 29(4): 231-234.

Badenes-Perez FR, Gershenzon J, Heckel DG. 2014. Insect attraction versus plant defense: young leaves high in glucosinolates stimulate oviposition by a specialist herbivore despite poor larval survival due to high saponin content. PLOS One 9(4): e95766.

Badenes-Pérez FR, Reichelt M, Gershenzon J, Heckel DG. 2010. Phylloplane location of glucosinolates in Barbarea spp. (Brassicaceae) and misleading assessment of host suitability by a specialist herbivore. New Phytologist 189(2): 549-556.

Baldwin IT. 1998. Jasmonate-induced responses are costly but benefit plants under attack in native populations. Proceedings of the National Academy of Sciences 95(14): 8113-8118.

Baldwin IT, Schultz JC. 1983. Rapid changes in tree leaf chemistry induced by damage: evidence for communication between plants. Science 221(4607): 277-279.

Bale JS, Masters GJ, Hodkinson ID, Awmack C, Bezemer TM, Brown VK, Butterfield J, Buse A, Coulson JC, Farrar J, et al. 2002. Herbivory in global climate change research: direct effects of rising temperature on insect herbivores. Global Change Biology 8(1): 1-16.

Ballaré CL. 2009. Illuminated behaviour: phytochrome as a key regulator of light foraging and plant antiherbivore defence. Plant Cell and Environment 32(6): 713-725.

Ballaré CL. 2011. Jasmonate-induced defenses: a tale of intelligence, collaborators and rascals. Trends in Plant Science 16(5): 249-257.

Ballaré CL. 2014. Light regulation of plant defense. Annual review of plant biology 65(1): 335-363.

Ballaré CL, Mazza CA, Austin AT, Pierik R. 2012. Canopy light and plant health. Plant Physiology 160(1): 145-155.

Ballaré CL, Pierik R. 2017. The shade-avoidance syndrome: multiple signals and ecological consequences. Plant, Cell \& Environment 40(11): 2530-2543.

Ballaré CL, Scopel AL, Sanchez RA. 1989. Photomodulation of axis extension in sparse canopies: role of the stem in the perception of light-quality signals of stand density. Plant Physiology 89(4): 1324-1330.

Ballaré CL, Scopel AL, Sanchez RA. 1990. Far-red radiation reflected from adjacent leaves: an early signal of competition in plant canopies. Science 247(4940): 329-332.

Barillot R, Escobar-Gutierrez AJ, Fournier C, Huynh P, Combes D. 2014. Assessing the effects of architectural variations on light partitioning within virtual wheat-pea mixtures. Annals of Botany 114(4): 725-737.

Barto EK, Cipollini D. 2005. Testing the optimal defense theory and the growth-differentiation balance hypothesis in Arabidopsis thaliana. Oecologia 146(2): 169-178.

Barton KE, Boege K. 2017. Future directions in the ontogeny of plant defence: understanding the evolutionary causes and consequences. Ecology Letters 20(4): 403-411.

Barton KE, Koricheva J. 2010. The ontogeny of plant defense and herbivory: characterizing general patterns using meta-analysis. American Naturalist 175(4): 481-493.

Bekaert M, Edger PP, Hudson CM, Pires JC, Conant GC. 2012. Metabolic and evolutionary costs of herbivory 
defense: systems biology of glucosinolate synthesis. New Phytologist 196(2): 596-605.

Bennett RN, Wallsgrove RM. 1994. Secondary metabolites in plant defense-mechanisms. New Phytologist 127(4): 617-633.

Bentley S, Whittaker JB. 1979. Effects of grazing by a chrysomelid beetle, gastrophysa viridula, on competition between Rumex obtusifolius and Rumex crispus. Journal of Ecology 67(1): 79-90.

Berendsen RL, Pieterse CMJ, Bakker PAHM. 2012. The rhizosphere microbiome and plant health. Trends in Plant Science 17(8): 478-486.

Berendsen RL, Vismans G, Yu K, Song Y, de Jonge R, Burgman WP, Burmolle M, Herschend J, Bakker P, Pieterse CMJ. 2018. Disease-induced assemblage of a plant-beneficial bacterial consortium. The ISME Journal 12: 1496-1507.

Berger S, Bell E, Sadka A, Mullet JE. 1995. Arabidopsis thaliana Atvsp is homologous to soybean VspA and VspB, genes encoding vegetative storage protein acid phosphatases, and is regulated similarly by methyl jasmonate, wounding, sugars, light and phosphate. Plant Molecular Biology 27(5): 933-942.

Bisseling T, Scheres B. 2014. Nutrient computation for root architecture. Science 346(6207): 300-301.

Bloom AJ, Chapin FS, Mooney HA. 1985. Resource limitation in plants-an economic analogy. Annual Review of Ecology and Systematics 16(1): 363-392.

Boege K, Marquis RJ. 2005. Facing herbivory as you grow up: the ontogeny of resistance in plants. Trends in Ecology \& Evolution 20(8): 441-448.

Bongers FJ. 2017. How virtual shade sheds light on plant plasticity. Wageningen University.

Bongers FJ, Evers JB, Anten NPR, Pierik R. 2014. From shade avoidance responses to plant performance at vegetation level: using virtual plant modelling as a tool. New Phytologist 204(2): 268-272.

Bongers FJ, Pierik R, Anten NPR, Evers JB. 2018. Subtle variation in shade avoidance responses may have profound consequences for plant competitiveness. Annals of Botany 121(5): 863-873.

Boonman A, Anten NP, Dueck TA, Jordi WJ, van der Werf A, Voesenek LA, Pons TL. 2006. Functiona significance of shade-induced leaf senescence in dense canopies: an experimental test using transgenic tobacco. American Naturalist 168(5): 597-607.

Borgström P, Strengbom J, Viketoft M, Bommarco R. 2016. Aboveground insect herbivory increases plant competitive asymmetry, while belowground herbivory mitigates the effect. Peer] 4: e1867.

Bradshaw AD, Caspari EW, Thoday JM 1965. Evolutionary significance of phenotypic plasticity in plants. Advances in Genetics: Academic Press, 115-155.

Broekgaarden C, Voorrips RE, Dicke M, Vosman B. 2011. Transcriptional responses of Brassica nigra to feeding by specialist insects of different feeding guilds. Insect science 18(3): 259-272.

Bruessow F, Gouhier-Darimont C, Buchala A, Metraux JP, Reymond P. 2010. Insect eggs suppress plant defence against chewing herbivores. The Plant Journal 62(5): 876-885.

Bruin J, Dicke M. 2001. Chemical information transfer between wounded and unwounded plants: backing up the future. Biochemical Systematics and Ecology 29(10): 1103-1113.

Brütting C, Schäfer M, Vanková R, Gase K, Baldwin IT, Meldau S. 2017. Changes in cytokinins are sufficient to alter developmental patterns of defense metabolites in Nicotiana attenuata. The Plant Journal 89(1): $15-30$.

Bryant JP, Chapin FS, Klein DR. 1983. Carbon/nutrient balance of boreal plants in relation to vertebrate herbivory. Oikos 40(3): 357-368.

Bukovinszky T, Gols R, Kamp A, de Oliveira-Domingues F, Hambäck PA, Jongema Y, Bezemer TM, Dicke M, van Dam NM, Harvey JA. 2010. Combined effects of patch size and plant nutritional quality on local densities of insect herbivores. Basic and Applied Ecology 11(5): 396-405.

Burdon JJ, Chilvers GA. 1982. Host density as a factor in plant-disease ecology. Annual Review of Phytopathology 20: 143-166

Bustos-Segura C, Poelman EH, Reichelt M, Gershenzon J, Gols R. 2017. Intraspecific chemical diversity among neighbouring plants correlates positively with plant size and herbivore load but negatively with herbivore damage. Ecology Letters 20(1): 87-97.

Campos ML, Yoshida Y, Major IT, Ferreira DD, Weraduwage SM, Froehlich JE, Johnson BF, Kramer DM, Jander G, Sharkey TD, et al. 2016. Rewiring of jasmonate and phytochrome B signalling uncouples plant growth-defense tradeoffs. Nature Communications $\mathbf{7}$.

Cates RG. 1980. Feeding patterns of monophagous, oligophagous, and polyphagous insect herbivores: The effect of resource abundance and plant chemistry. Oecologia 46(1): 22-31.

Cerrudo I, Keller MM, Cargnel MD, Demkura PV, de Wit M, Patitucci MS, Pierik R, Pieterse CM, Ballaré CL. 2012. Low red/far-red ratios reduce Arabidopsis resistance to Botrytis cinerea and jasmonate responses via a COI1-JAZ10-dependent, salicylic acid-independent mechanism. Plant Physiology 158(4): 2042-2052.

Chen BJW, During HJ, Vermeulen PJ, Kroon H, Poorter H, Anten NPR. 2015. Corrections for rooting volume and plant size reveal negative effects of neighbour presence on root allocation in pea. Functional Ecology 29(11): 1383-1391.

Chen T-W, Henke M, De Visser PH, Buck-Sorlin G, Wiechers D, Kahlen K, Stützel H. 2014. What is the most prominent factor limiting photosynthesis in different layers of a greenhouse cucumber canopy? Annals of Botany 114(4): 677-688.

Cipollini D. 2007. Consequences of the overproduction of methyl jasmonate on seed production, tolerance to defoliation and competitive effect and response of Arabidopsis thaliana. New Phytologist 173(1): 146153.

Cipollini D, Walters D, Voelckel C 2014. Costs of resistance in plants: from theory to evidence. Annual Plant Reviews: John Wiley \& Sons, Ltd, 263-307.

Conrath U, Beckers GJ, Flors V, Garcia-Agustin P, Jakab G, Mauch F, Newman MA, Pieterse CM, Poinssot B, Pozo MJ, et al. 2006. Priming: getting ready for battle. Molecular Plant-Microbe 
Interactions 19(10): 1062-1071.

Cortes LE, Weldegergis BT, Boccalandro HE, Dicke M, Ballaré CL. 2016. Trading direct for indirect defense? Phytochrome $B$ inactivation in tomato attenuates direct anti-herbivore defenses whilst enhancing volatile-mediated attraction of predators. New Phytologist 212(4): 1057-1071.

de Vries J, Evers JB, Dicke M, Poelman EH. 2018a. Ecological interactions shape the adaptive value of plant defence: herbivore attack versus competition for light. Functional Ecology in press.

de Vries J, Evers JB, Poelman EH. 2017. Dynamic plant-plant-herbivore interactions govern plant growthdefence integration. Trends in Plant Science 22(4): 329-337.

de Vries J, Poelman EH, Anten NP, Evers JB. 2018b. Elucidating the interaction between light competition and herbivore feeding patterns using functional-structural plant modelling. Annals of Botany 121(5): 1019-1031.

de Wit M, Kegge W, Evers JB, Eijk MHV, Gankema P, Voesenek LACJ, Pierik R. 2012. Plant neighbor detection through touching leaf tips precedes phytochrome signals. Proceedings of the National Academy of Sciences 109(36): 14705-14710.

de Wit M, Spoel SH, Sanchez-Perez GF, Gommers CMM, Pieterse CMJ, Voesenek LACJ, Pierik R. 2013. Perception of low red:far-red ratio compromises both salicylic acid- and jasmonic acid-dependent pathogen defences in Arabidopsis. The Plant Journal 75(1): 90-103.

Dewar RC, Franklin O, Mäkelä A, McMurtrie RE, Valentine HT. 2009. Optimal function explains forest responses to global change. Bioscience 59(2): 127-139.

Dicke M, Hilker M. 2003. Induced plant defences: from molecular biology to evolutionary ecology. Basic and Applied Ecology 4(1): 3-14.

Diggle AJ. 1988. ROOTMAP-a model in three-dimensional coordinates of the growth and structure of fibrous root systems. Plant and Soil 105(2): 169-178.

Domagalska MA, Leyser 0. 2011. Signal integration in the control of shoot branching. Nature Reviews Molecular Cell Biology 12(4): 211-221.

Douma JC, Vermeulen PJ, Poelman EH, Dicke M, Anten NPR. 2017. When does it pay off to prime for defense? A modeling analysis. New Phytologist 216(3): 782-797.

Dunbabin VM, Postma JA, Schnepf A, Pagès L, Javaux M, Wu L, Leitner D, Chen YL, Rengel Z, Diggle AJ. 2013. Modelling root-soil interactions using three-dimensional models of root growth, architecture and function. Plant and Soil 372(1-2): 93-124.

Dybzinski R, Farrior C, Wolf A, Reich PB, Pacala SW. 2011. Evolutionarily stable strategy carbon allocation to foliage, wood, and fine roots in trees competing for light and nitrogen: an analytically tractable, individual-based model and quantitative comparisons to data. The American Naturalist 177(2): 153166.

Ecker JR, Davis RW. 1987. Plant defense genes are regulated by ethylene. Proceedings of the National Academy of Sciences 84(15): 5202-5206.

Erb M. 2018. Plant Defenses against Herbivory: Closing the Fitness Gap. Trends in Plant Science 23(3): 187194.

Evers J, Vos J, Yin X, Romero P, Van Der Putten P, Struik P. 2010. Simulation of wheat growth and development based on organ-level photosynthesis and assimilate allocation. Journal of Experimental Botany 61(8): 2203-2216.

Evers JB 2016. Simulating crop growth and development using functional-structural plant modeling. In: Hikosaka K, Niinemets Ü, Anten PRN eds. Canopy Photosynthesis: From Basics to Applications. Dordrecht: Springer Netherlands, 219-236.

Evers JB, Bastiaans L. 2016. Quantifying the effect of crop spatial arrangement on weed suppression using functional-structural plant modelling. Journal of plant research 129(3): 339-351.

Evers JB, Letort V, Renton M, Kang M. 2018. Computational botany: advancing plant science through functional-structural plant modelling. Annals of Botany 121(5): 767-772.

Evers JB, van der Krol AR, Vos J, Struik PC. 2011. Understanding shoot branching by modelling form and function. Trends in Plant Science 16(9): 464-467.

Evers JB, Vos J, Chelle M, Andrieu B, Fournier C, Struik PC. 2007. Simulating the effects of localized red:farred ratio on tillering in spring wheat (Triticum aestivum) using a three-dimensional virtual plant model. New Phytologist 176(2): 325-336.

Fagerstrom T, Larsson S, Tenow 0. 1987. On optimal defence in plants. Functional Ecology 1(2): 73-81.

Falster DS, Westoby M. 2003. Plant height and evolutionary games. Trends in Ecology \& Evolution 18(7): 337343.

Faverjon L, Escobar-Gutiérrez A, Litrico I, Julier B, Louarn G. 2018. A generic individual-based model can predict yield, nitrogen content, and species abundance in experimental grassland communities. Journal of Experimental Botany.

Feeny P 1976. Plant apparency and chemical defense. In: Wallace JW, Mansell RL eds. Biochemical Interaction Between Plants and Insects. Boston, MA: Springer US, 1-40.

Felton GW, Tumlinson JH. 2008. Plant-insect dialogs: complex interactions at the plant-insect interface. Current Opinion in Plant Biology 11(4): 457-463.

Fineblum WL, Rausher MD. 1995. Tradeoff between resistance and tolerance to herbivore damage in a morning glory. Nature 377: 517.

Franklin KA. 2008. Shade avoidance. New Phytologist 179(4): 930-944.

Fraser DP, Hayes S, Franklin KA. 2016. Photoreceptor crosstalk in shade avoidance. Current Opinion in Plant Biology 33: 1-7.

Freckleton RP, Watkinson AR. 2001. Asymmetric competition between plant species. Functional Ecology 15(5): 615-623.

Fry JD. 1992. The mixed-model analysis of variance applied to quantitative genetics: biological meaning of the 
parameters. Evolution 46(2): 540-550.

Gersani M, Brown Joel S, O'Brien Erin E, Maina Godfrey M, Abramsky Z. 2001. Tragedy of the commons as a result of root competition. Journal of Ecology 89(4): 660-669.

Gershenzon J 1994. The cost of plant chemical defense against herbivory: a biochemical perspective. In: Bernays EA ed. Insect-Plant Interactions. Boca Raton, FL: CRC Press, 105-173.

Giron D, Frago E, Glevarec G, Pieterse CMJ, Dicke M. 2013. Cytokinins as key regulators in plant-microbeinsect interactions: connecting plant growth and defence. Functional Ecology 27(3): 599-609.

Givnish TJ 1995. 1 - Plant stems: biomechanical adaptation for energy capture and influence on species distributions A2 - Gartner, Barbara L. Plant Stems. San Diego: Academic Press, 3-49.

Glazebrook J. 2005. Contrasting mechanisms of defense against biotrophic and necrotrophic pathogens. Annual Review of Phytopathology 43: 205-227.

Gols R, Wagenaar R, Bukovinszky T, van Dam NM, Dicke M, Bullock JM, Harvey JA. 2008. Genetic variation in defense chemistry in wild cabbages affects herbivores and their endoparasitoids. Ecology 89(6): 1616-1626.

Haag JJ, Coupe MD, Cahill JF. 2004. Antagonistic interactions between competition and insect herbivory on plant growth. Journal of Ecology 92(1): 156-167.

Hambäck PA, Beckerman AP. 2003. Herbivory and plant resource competition: A review of two interacting interactions. Oikos 101(1): 26-37.

Hättenschwiler S, Vitousek PM. 2000. The role of polyphenols in terrestrial ecosystem nutrient cycling. Trends in Ecology \& Evolution 15(6): 238-243.

Heidel-Fischer HM, Musser RO, Vogel H. 2014. Plant transcriptomic responses to herbivory. Annual Plant Reviews: Insect-Plant Interactions 47: 155-196.

Heil M. 2002. Ecological costs of induced resistance. Current Opinion in Plant Biology 5(4): 345-350.

Heil M, Baldwin IT. 2002. Fitness costs of induced resistance: emerging experimental support for a slippery concept. Trends in Plant Science 7(2): 61-67.

Heil M, Hilpert A, Kaiser W, Linsenmair KE. 2000. Reduced growth and seed set following chemical induction of pathogen defence: does systemic acquired resistance (SAR) incur allocation costs? Journal of Ecology 88(4): 645-654.

Heil M, Ton J. 2008. Long-distance signalling in plant defence. Trends in Plant Science 13(6): 264-272.

Hemmerling R, Kniemeyer O, Lanwert D, Kurth W, Buck-Sorlin G. 2008. The rule-based language XL and the modelling environment GroIMP illustrated with simulated tree competition. Functional Plant Biology 35(9-10): 739-750.

Herms DA, Mattson WJ. 1992. The dilemma of plants: to grow or defend. The Quarterly Review of Biology 67(3): 283-335.

Heuvelink E. 1996. Dry matter partitioning in tomato: validation of a dynamic simulation model. Annals of Botany 77(1): 71-80.

Hikosaka K, Anten NPR, Borjigidai A, Kamiyama C, Sakai H, Hasegawa T, Oikawa S, Iio A, Watanabe M, Koike T, et al. 2016. A meta-analysis of leaf nitrogen distribution within plant canopies. Annals of Botany 118(2): 239-247.

Hirose T. 2005. Development of the Monsi-Saeki theory on canopy structure and function. Annals of Botany 95(3): 483-494.

Hirose T, Werger MJA, Pons TL, Rheenen JWA. 1987. Canopy structure and leaf nitrogen distribution in a stand of Lysimachia vulgaris $L$. as influenced by stand density. Oecologia 77(2): 145-150.

Hochwender CG, Marquis RJ, Stowe KA. 2000. The potential for and constraints on the evolution of compensatory ability in Asclepias syriaca. Oecologia 122(3): 361-370.

Howe GA, Jander G. 2008. Plant immunity to insect herbivores. Annual review of plant biology 59: 41-66.

Izaguirre M, Mazza C, Astigueta M, Ciarla A, Ballaré C. 2013. No time for candy: passionfruit (Passiflora edulis) plants down-regulate damage-induced extra floral nectar production in response to light signals of competition. Oecologia 173(1): 213-221.

Jacquemoud S, Baret F. 1990. PROSPECT: A model of leaf optical properties spectra. Remote Sensing of Environment 34(2): 75-91.

Jaenike J. 1990. Host specialization in phytophagous insects. Annual Review of Ecology and Systematics 21(1): 243-273.

Jing J, Raaijmakers C, Kostenko O, Kos M, Mulder PPJ, Bezemer TM. 2015. Interactive effects of aboveand belowground herbivory and plant competition on plant growth and defence. Basic and Applied Ecology 16(6): 500-509.

Johnson MTJ, Agrawal AA. 2005. Plant genotype and environment interact to shape a diverse arthropod community on evening primrose (Oenothera biennis). Ecology 86(4): 874-885.

Jones T, Kulseth S, Mechtenberg K, Jorgenson C, Zehfus M, Brown P, Siemens DH. 2006. Simultaneous evolution of competitiveness and defense: induced switching in Arabis drummondii. Plant Ecology 184(2): 245-257.

Joshi J, Otway SJ, Koricheva J, Pfisterer AB, Alphei J, Roy BA, Scherer-Lorenzen M, Schmid B, Spehn E, Hector A 2008. Bottom-up effects and feedbacks in simple and diverse experimental grassland communities. In: Weisser WW, Siemann E eds. Insects and Ecosystem Function. Berlin, Heidelberg: Springer Berlin Heidelberg, 115-135.

Kang M, Heuvelink E, Carvalho SMP, de Reffye P. 2012. A virtual plant that responds to the environment like a real one: the case for chrysanthemum. New Phytologist 195(2): 384-395.

Karban R, Yang LH, Edwards KF. 2013. Volatile communication between plants that affects herbivory: a metaanalysis. Ecology Letters 17(1): 44-52.

Kegge W, Ninkovic V, Glinwood R, Welschen RAM, Voesenek LACJ, Pierik R. 2015. Red:far-red light conditions affect the emission of volatile organic compounds from barley (Hordeum vulgare), leading to 
altered biomass allocation in neighbouring plants. Annals of Botany 116(5): 845.

Kegge W, Pierik R. 2010. Biogenic volatile organic compounds and plant competition. Trends in Plant Science 15(3): 126-132.

Kegge W, Weldegergis BT, Soler R, Eijk MV-V, Dicke M, Voesenek LACJ, Pierik R. 2013. Canopy light cues affect emission of constitutive and methyl jasmonate-induced volatile organic compounds in Arabidopsis thaliana. New Phytologist 200(3): 861-874.

Kessler A. 2015. The information landscape of plant constitutive and induced secondary metabolite production. Current Opinion in Insect Science 8: 47-53.

Kessler A, Halitschke R, Diezel C, Baldwin IT. 2006. Priming of plant defense responses in nature by airborne signaling between Artemisia tridentata and Nicotiana attenuata. Oecologia 148(2): 280-292.

Keuskamp DH, Keller MM, Ballaré CL, Pierik R. 2012. Blue light regulated shade avoidance. Plant Signal and Behaviour 7(4): 514-517.

Kim TN, Underwood N, Inouye BD. 2013. Insect herbivores change the outcome of plant competition through both inter- and intraspecific processes. Ecology 94(8): 1753-1763.

King DA. 1990. The adaptive significance of tree height. The American Naturalist 135(6): 809-828.

Koricheva J. 2002. Meta-analysis of sources of variation in fitness costs of plant antiherbivore defenses. Ecology 83(1): 176-190.

Koricheva J, Barton KE 2012. Temporal changes in plant secondary metabolite production: patterns, causes and consequences. In: Iason GR, Dicke M, Hartley SE eds. The Ecology of Plant Secondary Metabolites: From Genes to Global Processes: Cambridge University Press, 34-55.

Krimmel B, Pearse IS. 2016. Tolerance and phenological avoidance of herbivory in tarweed species. Ecology 97(5): 1357-1363.

Kroes A, Stam JM, David A, Boland W, Loon JJA, Dicke M, Poelman EH. 2016. Plant-mediated interactions between two herbivores differentially affect a subsequently arriving third herbivore in populations of wild cabbage. Plant Biology 18(6): 981-991.

Lambdon PW, Hassall M, Boar RR, Mithen R. 2003. Asynchrony in the nitrogen and glucosinolate leaf-age profiles of Brassica: is this a defensive strategy against generalist herbivores? Agriculture, Ecosystems \& Environment 97(1): 205-214.

Lankau RA. 2007. Specialist and generalist herbivores exert opposing selection on a chemical defense. New Phytologist 175(1): 176-184.

Lankau RA, Kliebenstein DJ. 2009. Competition, herbivory and genetics interact to determine the accumulation and fitness consequences of a defence metabolite. Journal of Ecology 97(1): 78-88.

Lankau RA, Strauss SY. 2008. Community complexity drives patterns of natural selection on a chemical Defense of Brassica nigra. American Naturalist 171(2): 150-161.

Ledo A, Paul KI, Burslem DFRP, Ewel JJ, Barton C, Battaglia M, Brooksbank K, Carter J, Eid TH, England JR, et al. 2018. Tree size and climatic water deficit control root to shoot ratio in individual trees globally. New Phytologist 217(1): 8-11.

Leone M, Keller MM, Cerrudo I, Ballaré CL. 2014. To grow or defend? Low red: far-red ratios reduce jasmonate sensitivity in Arabidopsis seedlings by promoting DELLA degradation and increasing JAZ10 stability. New Phytologist 204(2): 355-367.

Lim PO, Kim HJ, Gil Nam H. 2007. Leaf senescence. Annual review of plant biology 58(1): 115-136.

Lin T, Klinkhamer PGL, Vrieling K. 2015. Parallel evolution in an invasive plant: effect of herbivores on competitive ability and regrowth of Jacobaea vulgaris. Ecology Letters 18(7): 668-676.

Lopez G, Favreau RR, Smith C, DeJong TM. 2010. L-PEACH: a computer-based model to understand how peach trees grow. HortTechnology 20(6): 983-990.

Louarn G, Faverjon L. 2018. A generic individual-based model to simulate morphogenesis, $\mathrm{C}-\mathrm{N}$ acquisition and population dynamics in contrasting forage legumes. Annals of Botany 121(5): 875-896.

Lynch J, Nielsen K, Davis R, Jablokow A. 1997. SimRoot: Modelling and visualization of root systems. Plant and Soil 188(1): 139-151.

Lynch JP. 2013. Steep, cheap and deep: an ideotype to optimize water and $\mathrm{N}$ acquisition by maize root systems. Annals of Botany 112(2): 347-357.

Malagoli P, Lainé P, Le Deunff E, Rossato L, Ney B, Ourry A. 2004. Modeling nitrogen uptake in oilseed rape cv Capitol during a growth cycle using influx kinetics of root nitrate transport systems and field experimental data. Plant Physiology 134(1): 388-400.

Maynard Smith J. 1982. Evolution and the Theory of Games: Cambridge University Press.

Mazza CA, Ballaré CL. 2015. Photoreceptors UVR8 and phytochrome B cooperate to optimize plant growth and defense in patchy canopies. New Phytologist 207(1): 4-9.

McArt SH, Halitschke R, Salminen J-P, Thaler JS. 2013. Leaf herbivory increases plant fitness via induced resistance to seed predators. Ecology 94(4): 966-975.

McKey D. 1974. Adaptive patterns in alkaloid physiology. The American Naturalist 108(961): 305-320.

McNickle GG, Dybzinski R. 2013. Game theory and plant ecology. Ecology Letters 16(4): 545-555.

McNickle GG, Evans WD. 2018. Toleration games: compensatory growth by plants in response to enemy attack is an evolutionarily stable strategy. AOB PLANTS 10(4): ply035-ply035.

Mommer L, Kirkegaard J, van Ruijven J. 2016. Root-root interactions: towards a rhizosphere framework. Trends in Plant Science 21(3): 209-217.

Mommer L, van Ruijven J, Jansen C, van de Steeg HM, de Kroon H. 2012. Interactive effects of nutrient heterogeneity and competition: implications for root foraging theory? Functional Ecology 26(1): 66-73.

Moreno JE, Tao Y, Chory J, Ballaré CL. 2009. Ecological modulation of plant defense via phytochrome control of jasmonate sensitivity. Proceedings of the National Academy of Sciences 106(12): 4935-4940.

Musser RO, Hum-Musser SM, Eichenseer H, Peiffer M, Ervin G, Murphy JB, Felton GW. 2002. Caterpillar saliva beats plant defences. Nature 416: 599. 
Nabity PD, Zavala JA, DeLucia EH. 2009. Indirect suppression of photosynthesis on individual leaves by arthropod herbivory. Annals of Botany 103(4): 655-663.

Neilson EH, Goodger JQD, Woodrow IE, Møller BL. 2013. Plant chemical defense: at what cost? Trends in Plant Science 18(5): 250-258.

Niinemets Ü, Anten NPR 2009. Packing the photosynthetic machinery: from leaf to canopy. In: Laisk A, Nedbal $\mathrm{L}$, Govindjee eds. Photosynthesis in silico : Understanding Complexity from Molecules to Ecosystems. Dordrecht: Springer Netherlands, 363-399.

O'Brien EE, Brown JS, Moll JD. 2007. Roots in space: a spatially explicit model for below-ground competition in plants. Proceedings of the Royal Society B: Biological Sciences 274(1612): 929.

O'Brien EE, Gersani M, Brown JS. 2005. Root proliferation and seed yield in response to spatial heterogeneity of below-ground competition. New Phytologist 168(2): 401-412.

Ohnmeiss TE, Baldwin IT. 2000. Optimal defense theory predicts the ontogeny of an induced nicotine defense. Ecology 81(7): 1765-1783.

Oksanen L 1990. Predation, herbivory, and plant strategies along gradients of primary productivity. San Diego, California: Academic Press, Inc. 445-474.

Otway SJ, Hector A, lawton JH. 2005. Resource dilution effects on specialist insect herbivores in a grassland biodiversity experiment. Journal of Animal Ecology 74(2): 234-240.

Pagès L, Jordan MO, Picard D. 1989. A simulation model of the three-dimensional architecture of the maize root system. Plant and Soil 119(1): 147-154.

Pantazopoulou CK, Bongers FJ, Küpers JJ, Reinen E, Das D, Evers JB, Anten NPR, Pierik R. 2017. Neighbor detection at the leaf tip adaptively regulates upward leaf movement through spatial auxin dynamics. Proceedings of the National Academy of Sciences 114(28): 7450-7455.

Parker GA, Smith JM. 1990. Optimality theory in evolutionary biology. Nature 348: 27.

Perez RPA, Dauzat J, Pallas B, Lamour J, Verley P, Caliman J-P, Costes E, Faivre R. 2018. Designing oil palm architectural ideotypes for optimal light interception and carbon assimilation through a sensitivity analysis of leaf traits. Annals of Botany 121(5): 909-926.

Philippot L, Raaijmakers JM, Lemanceau P, van der Putten WH. 2013. Going back to the roots: the microbial ecology of the rhizosphere. Nature reviews. Microbiology 11(11): 789-799.

Pierik R, Ballaré CL, Dicke M. 2014. Ecology of plant volatiles: taking a plant community perspective. Plant Cell and Environment 37(8): 1845-1853.

Pierik R, Cuppens MLC, Voesenek LACJ, Visser EJW. 2004a. Interactions between ethylene and gibberellins in phytochrome-mediated shade avoidance responses in tobacco. Plant Physiology 136(2): 2928.

Pierik R, de Wit M. 2014. Shade avoidance: phytochrome signalling and other aboveground neighbour detection cues. Journal of Experimental Botany 65(11): 2815-2824.

Pierik R, Mommer L, Voesenek LACJ. 2013. Molecular mechanisms of plant competition: neighbour detection and response strategies. Functional Ecology 27(4): 841-853.

Pierik R, Whitelam GC, Voesenek LACJ, De Kroon H, Visser EJW. 2004b. Canopy studies on ethyleneinsensitive tobacco identify ethylene as a novel element in blue light and plant-plant signalling. The Plant Journal 38(2): 310-319.

Pieterse CM, Van der Does D, Zamioudis C, Leon-Reyes A, Van Wees SC. 2012. Hormonal modulation of plant immunity. Annual Review of Cell and Developmental Biology 28: 489-521.

Poelman EH. 2015. From induced resistance to defence in plant-insect interactions. Entomologia Experimentalis Et Applicata 157(1): 11-17.

Poelman EH, Broekgaarden C, Van Loon JJA, Dicke M. 2008a. Early season herbivore differentially affects plant defence responses to subsequently colonizing herbivores and their abundance in the field. Molecular Ecology 17(14): 3352-3365.

Poelman EH, Dicke M 2014. Plant-mediated interactions among insects within a community ecological perspective. Annual Plant Reviews: John Wiley \& Sons, Ltd, 309-337.

Poelman EH, Kessler A. 2016. Keystone herbivores and the evolution of plant defenses. Trends in Plant Science 21(6): 477-485.

Poelman EH, van Dam NM, van Loon JJA, Vet LEM, Dicke M. 2009. Chemical diversity in Brassica oleracea affects biodiversity of insect herbivores. Ecology 90(7): 1863-1877.

Poelman EH, van Loon JJA, Dicke M. 2008b. Consequences of variation in plant defense for biodiversity at higher trophic levels. Trends in Plant Science 13(10): 534-541.

Poelman EH, Van Loon JJA, Van Dam NM, Vet LEM, Dicke M. 2010. Herbivore-induced plant responses in Brassica oleracea prevail over effects of constitutive resistance and result in enhanced herbivore attack. Ecological Entomology 35(2): 240-247.

Poorter H, Nagel O. 2000. The role of biomass allocation in the growth response of plants to different levels of light, CO2, nutrients and water: a quantitative review. Functional Plant Biology 27(12): 1191-1191.

Poorter H, Niklas KJ, Reich PB, Oleksyn J, Poot P, Mommer L. 2012. Biomass allocation to leaves, stems and roots: meta-analyses of interspecific variation and environmental control. New Phytologist 193(1): 30-50.

Postma JA, Lynch JP. 2012. Complementarity in root architecture for nutrient uptake in ancient maize/bean and maize/bean/squash polycultures. Annals of Botany 110(2): 521-534.

Qi J, Zhang M, Lu C, Hettenhausen C, Tan Q, Cao G, Zhu X, Wu G, Wu J. 2018. Ultraviolet-B enhances the resistance of multiple plant species to lepidopteran insect herbivory through the jasmonic acid pathway. Scientific Reports 8(1): 277.

Rasmussen CR, Weisbach AN, Thorup-Kristensen K, Weiner J. 2017. Size-asymmetric root competition in deep, nutrient-poor soil. Journal of Plant Ecology: rtx064-rtx064.

Ratnadass A, Fernandes P, Avelino J, Habib R. 2012. Plant species diversity for sustainable management of crop pests and diseases in agroecosystems: a review. Agronomy for Sustainable Development $\mathbf{3 2}(1)$ : 
273-303.

Rees M, Brown VK. 1992. Interactions between Invertebrate Herbivores and Plant Competition. Journal of Ecology 80(2): 353-360.

Renton M, Poot P. 2014. Simulation of the evolution of root water foraging strategies in dry and shallow soils. Annals of Botany 114(4): 763-778.

Rhainds M, English-Loeb G. 2003. Testing the resource concentration hypothesis with tarnished plant bug on strawberry: density of hosts and patch size influence the interaction between abundance of nymphs and incidence of damage. Ecological Entomology 28(3): 348-358.

Richards CL, Bossdorf O, Muth NZ, Gurevitch J, Pigliucci M. 2006. Jack of all trades, master of some? On the role of phenotypic plasticity in plant invasions. Ecology Letters 9(8): 981-993.

Rosenthal JP, Kotanen PM. 1994. Terrestrial plant tolerance to herbivory. Trends in Ecology \& Evolution 9(4): $145-148$

Rossato L, Lainé P, Ourry A. 2001. Nitrogen storage and remobilization in Brassica napus L. during the growth cycle: nitrogen fluxes within the plant and changes in soluble protein patterns. Journal of Experimental Botany 52(361): 1655-1663.

Schadler M, Brandl R, Haase J. 2007. Antagonistic interactions between plant competition and insect herbivory. Ecology 88(6): 1490-1498.

Schmidt DD, Voelckel C, Hartl M, Schmidt S, Baldwin IT. 2005. Specificity in ecological interactions. Attack from the same lepidopteran herbivore results in species-specific transcriptional responses in two solanaceous host plants. Plant Physiology 138(3): 1763.

Schoonhoven LM, van Loon JJA, Dicke M. 2005. Insect-plant biology. Oxford: Oxford University Press.

Siddiqi MY, Glass ADM, Ruth TJ, Rufty TW. 1990. Studies of the uptake of nitrate in barley. Plant Physiology 93(4): 1426.

Sparkes DL, Holme SJ, Gaju 0. 2006. Does light quality initiate tiller death in wheat? European Journal of Agronomy 24(3): 212-217.

Spoel SH, Dong X. 2008. Making sense of hormone crosstalk during plant immune responses. Cell Host \& Microbe 3(6): 348-351.

Stam JM, Chrétien L, Dicke M, Poelman EH. 2017. Response of Brassica oleracea to temporal variation in attack by two herbivores affects preference and performance of a third herbivore. Ecological Entomology 42(6): 803-815

Stam JM, Kroes A, Li Y, Gols R, van Loon JJ, Poelman EH, Dicke M. 2014. Plant interactions with multiple insect herbivores: from community to genes. Annual review of plant biology 65: 689-713.

Stockhoff BA. 1994. Maximization of daily canopy photosynthesis - effects of herbivory on optimal nitrogen distribution. Journal of Theoretical Biology 169(3): 209-220.

Stotz HU, Koch T, Biedermann A, Weniger K, Boland W, Mitchell-Olds T. 2002. Evidence for regulation of resistance in Arabidopsis to Egyptian cotton worm by salicylic and jasmonic acid signaling pathways. Planta 214(4): 648-652.

Stowe KA. 1998. Experimental evolution of resistance in Brassica rapa: Correlated response of tolerance in lines selected for glucosinolate content. Evolution 52(3): 703-712.

Stowe KA, Marquis RJ, Hochwender CG, Simms EL. 2000. The evolutionary ecology of tolerance to consumer damage. Annual Review of Ecology and Systematics 31: 565-595.

Strauss SY, Agrawal AA. 1999. The ecology and evolution of plant tolerance to herbivory. Trends in Ecology \& Evolution 14(5): 179-185.

Strauss SY, Irwin RE. 2004. Ecological and evolutionary consequences of multispecies plant-animal interactions. Annual Review of Ecology, Evolution, and Systematics 35(1): 435-466.

Strauss SY, Rudgers JA, Lau JA, Irwin RE. 2002. Direct and ecological costs of resistance to herbivory. Trends in Ecology \& Evolution 17(6): 278-285.

Stuart J. 2015. Insect effectors and gene-for-gene interactions with host plants. Current Opinion in Insect Science 9: 56-61.

Sultan SE. 2000. Phenotypic plasticity for plant development, function and life history. Trends in Plant Science 5(12): 537-542.

Tack AJM, Dicke M. 2013. Plant pathogens structure arthropod communities across multiple spatial and temporal scales. Functional Ecology 27(3): 633-645.

Thaler JS, Humphrey PT, Whiteman NK. 2012. Evolution of jasmonate and salicylate signal crosstalk. Trends in Plant Science 17(5): 260-270.

Thornley JHM. 1972. A balanced quantitative model for root: shoot ratios in vegetative plants. Annals of Botany 36(2): 431-441.

Tilman D. 1988. Plant strategies and the dynamics and structure of plant communities. Monographs in Population Biology 26.

Trumble JT, Kolodny-Hirsch DM, Ting IP. 1993. Plant compensation for arthropod herbivory. Annual Review of Entomology 38(1): 93-119.

Tuomi J, Niemelä P, Stuart Chapin F, Bryant JP, Sirén S 1988. Defensive responses of trees in relation to their carbon/nutrient balance. New York, NY: Springer New York. 57-72.

Ullmann-Zeunert L, Stanton MA, Wielsch N, Bartram S, Hummert C, Svatoš A, Baldwin IT, Groten K. 2013. Quantification of growth-defense trade-offs in a common currency: nitrogen required for phenolamide biosynthesis is not derived from ribulose-1,5-bisphosphate carboxylase/oxygenase turnover. The Plant Journal 75(3): 417-429.

UIm R, Jenkins GI. 2015. Q\&A: How do plants sense and respond to UV-B radiation? BMC Biology 13(1): 45.

van Dam NM, Baldwin IT. 1998. Costs of jasmonate-induced responses in plants competing for limited resources. Ecology Letters 1(1): 30-33.

Van Dam NM, Baldwin IT. 2001. Competition mediates costs of jasmonate-induced defences, nitrogen 
acquisition and transgenerational plasticity in Nicotiana attenuata. Functional Ecology 15(3): 406-415.

Van Dam NM, De Jong TJ, Iwasa Y, Kubo T. 1996. Optimal distribution of defences: Are plants smart investors? Functional Ecology 10(1): 128-136.

van Dam NM, Raaijmakers CE. 2006. Local and systemic induced responses to cabbage root fly larvae (Delia radicum) in Brassica nigra and B. oleracea. CHEMOECOLOGY 16(1): 17-24.

van Dam NM, Witte L, Theuring C, Hartmann T. 1995. Distribution, biosynthesis and turnover of pyrrolizidine alkaloids in Cynoglossum officinale. Phytochemistry 39(2): 287-292.

Van Der Meijden E. 1996. Plant defence, an evolutionary dilemma: contrasting effects of (specialist and generalist) herbivores and natural enemies. Entomologia Experimentalis Et Applicata 80(1): 307-310.

van Hulten M, Pelser M, van Loon LC, Pieterse CMJ, Ton J. 2006. Costs and benefits of priming for defense in Arabidopsis. Proceedings of the National Academy of Sciences 103(14): 5602-5607.

Van Zandt PA, Agrawal AA. 2004. Community-wide impacts of herbivore-induced plant responses in milkweed (Asclepias syriaca). Ecology 85(9): 2616-2629.

Viswanathan DV, Narwani AJT, Thaler JS. 2005. Specificity in induced plant responses shapes patterns of herbivore occurrence on Solanum dulcamara. Ecology 86(4): 886-896.

Vos J, Evers JB, Buck-Sorlin GH, Andrieu B, Chelle M, de Visser PHB. 2010. Functional-structural plant modelling: a new versatile tool in crop science. Journal of Experimental Botany 61(8): 2101-2115.

Weech M-H, Chapleau M, Pan L, Ide C, Bede JC. 2008. Caterpillar saliva interferes with induced Arabidopsis thaliana defence responses via the systemic acquired resistance pathway. Journal of Experimental Botany 59(9): 2437-2448.

Wei X, Vrieling K, Mulder PPJ, Klinkhamer PGL. 2015. Testing the generalist-specialist dilemma: the role of pyrrolizidine alkaloids in resistance to invertebrate herbivores in Jacobaea species. Journal of Chemical Ecology 41(2): 159-167.

Weiner J. 1990. Asymmetric competition in plant populations. Trends in Ecology \& Evolution 5(11): 360-364.

Weinig C. 2007. Plasticity versus canalization: population differences in the timing of shade-avoidance responses. Evolution 54(2): 441-451.

Wheat CW, Vogel H, Wittstock U, Braby MF, Underwood D, Mitchell-Olds T. 2007. The genetic basis of a plant-insect coevolutionary key innovation. Proceedings of the National Academy of Sciences 104(51): 20427.

Whitham TG, Bailey JK, Schweitzer JA, Shuster SM, Bangert RK, Leroy CJ, Lonsdorf EV, Allan GJ, Difazio SP, Potts BM, et al. 2006. A framework for community and ecosystem genetics: from genes to ecosystems. Nature Reviews Genetics 7(7): 510-523.

Whitham TG, Gehring CA, Lamit LJ, Wojtowicz T, Evans LM, Keith AR, Smith DS. 2012. Community specificity: life and afterlife effects of genes. Trends in Plant Science 17(5): 271-281.

Yin X, Goudriaan JAN, Lantinga EA, Vos JAN, Spiertz HJ. 2003. A flexible sigmoid function of determinate growth. Annals of Botany 91(3): 361-371.

Yin X, Laar. 2005. Crop Systems Dynamics: An Ecophysiological Simulation Model for Genotype-by-Environment Interactions.

Yoshinaka K, Nagashima H, Yanagita Y, Hikosaka K. 2018. The role of biomass allocation between lamina and petioles in a game of light competition in a dense stand of an annual plant. Annals of Botany 121(5): $1055-1064$

Zangerl AR. 1986. Leaf value and optimal defense in Pastinaca sativa L. (Umbelliferae). The American Midland Naturalist 116(2): 432-436.

Zhang D-Y, Sun G-J, Jiang X-H. 1999. Donald's ideotype and growth redundancy: a game theoretical analysis. Field Crops Research 61(2): 179-187.

Zhang T, Maruhnich SA, Folta KM. 2011. Green light induces shade avoidance symptoms. Plant Physiology 157(3): 1528-1536.

Zhong X, Peng S, Sheehy JE, Vesperas RM, Liu H. 2002. Relationship between tillering and leaf area index: quantifying critical leaf area index for tillering in rice. Journal of Agricultural Science(138): 269-279.

Zhu J, van der Werf W, Anten NPR, Vos J, Evers JBC. 2015. The contribution of phenotypic plasticity to complementary light capture in plant mixtures. New Phytologist 207(4): 1213-1222.

Züst T, Agrawal AA. 2017. Trade-offs between plant growth and defense against insect herbivory: an emerging mechanistic synthesis. Annual review of plant biology 68: 513-534.

Züst T, Joseph B, Shimizu KK, Kliebenstein DJ, Turnbull LA. 2011. Using knockout mutants to reveal the growth costs of defensive traits. Proceedings of the Royal Society of London B: Biological Sciences 278(1718): 2598-2603. 


\section{English summary}

Plants grow in dynamic environments where their fitness is determined by a multitude of biotic and abiotic factors in the environment. These environments house complex webs of interactions in which plants must compete with neighbours for limited resources such as light, while having to defend against a multitude of heterotrophic organisms such as insect herbivores. Plants respond to cues related to neighbour presence and herbivore damage with a suite of induced shade avoidance and defence responses, respectively. One of the signals that interlinks these shade avoidance and defence responses is the red to far-red ratio (R:FR), which is a neighbour detection cue that induces shade avoidance responses and downregulates direct defence responses. This physiological linkage has been hypothesised as a mechanism that balances a resource allocation trade-off by promoting resource acquisition through competitive growth, at the expense of defence. However, the optimal expression of traits related to competitive growth and defence are not only dependent on biotic environmental factors, but also on interactions with the plant and herbivore communities. The aim of this thesis was to elucidate how ecological interactions shape the optimal balance between the acquisition and protection of resources. To reach this objective, I used a functional-structural plant (FSP) modelling approach to simulate physiological mechanisms that interact with the environment, how these mechanisms drive ecological interactions between plants and herbivores, and the evolutionary dynamics that arise from these interactions. In the general introduction (chapter 1), the linkage between competitive growth and defence is introduced from physiological, ecological and evolutionary perspectives, and FSP modelling is introduced as a tool to study the balance between competitive growth and defence. In this thesis, I describe and utilise two FSP models; an FSP model of Brassica nigra that focusses on detailed physiological responses and is parameterised and validated using field and greenhouse experiments, and an FSP model of a generic dicotyledon that focusses on evolutionary dynamics.

In chapter 2, I outline my view on using FSP modelling to study interactions between the plant and herbivore communities and accentuate the importance of considering these interactions in a dynamic environment. In this chapter, I propose four alternative hypotheses to the origin of the physiological linkage between shade avoidance and defence responses that go beyond the perspective of resource allocation trade-offs between growth and defence. The downregulation of direct defence by a low R:FR cue may function to (i) focus defence on 
valuable tissues, (ii) deter specialist herbivores, (iii) control interactions within the insect herbivore community or (iv) prevent the emission of volatile cues to which neighbouring plants may respond.

Chapter 3 presents an FSP model of B. nigra that combines mechanistic simulation of plant growth, shade avoidance responses and herbivore feeding, which are parameterised and validated using field and greenhouse experiments. This model is used to analyse the impact of herbivore feeding at different locations within the plant that relate to herbivore specialisation on plant fitness in a dynamic environment where plants compete for light. This analysis shows that herbivore damage had a larger effect on plant fitness when plants were competing for light and when the damage was directed at young leaves at the top of the canopy.

Chapter 4 expands on the FSP model presented in chapter 3 with the addition of plant defence, and is used to analyse how the direct costs (e.g. metabolic costs) and benefits of defence as well as indirect costs and benefits of defence imposed through ecological interactions impact plant fitness. This analysis shows that ecological costs of defence imposed by inter-genotypic competition is the strongest determinant of plant fitness, amplifying size differences resulting from differences in defence investment. Additionally, the model shows that the benefit of plant defence relies on reducing herbivore damage rather than dispersing herbivore damage away from young leaves and towards older leaves that are of less value to the plant.

Chapter 5 expands on the FSP model presented in chapter 4 with the addition of R:FR mediation of plant defence, to analyse the function of this physiological regulation from the perspective of simple versus competitive optimisation (e.g. mono-stands vs mixtures). The model results show that plant-level defence investment was a strong determinant of plant fitness, and that leaf-level mediation of plant defence by R:FR may provide an additional fitness benefit in high plant densities. Furthermore, the model shows that the optimal plant-level defence expression does not monotonically decrease with plant density. This indicates that $\mathrm{R}: F R$ mediation of defence alone is not sufficient to optimise plant-level defence between densities, as R:FR does monotonically decrease with plant density.

Chapter 6 presents a new FSP model that simulates the evolutionary dynamics of a plant population, driven by competition for light and nitrogen in combination with herbivore damage. This evolutionary FSP model is used to analyse how optimal biomass allocation patterns 
changed with plant density, nitrogen availability and herbivore damage, and how these factors impact the optimal balance between the acquisition and protection of resources. The model results conform with the functional equilibria described by optimal partitioning theory (OPT), which predicts an increase in biomass allocation towards plant parts that partake in the acquisition of a limiting resource. The model further shows that optimal defence levels negatively correlate with nitrogen availability, and positively correlate with plant defence if an increase in plant density decreases the nitrogen availability per plant. This chapter concludes that the adaptive value of plant defence is dependent on the availability of, and competition for both above- and belowground resources.

In the general discussion (chapter 7), I discuss the concepts and results presented in chapters 2-6 with respect to the main objective specified in the general introduction (chapter 1). First, I discuss how ecological interactions in a dynamic environment shape growth-defence integration and the challenges and opportunities of using FSP models to simulate these dynamic environments. To illustrate this I present additional simulations that show the effect of a variable plant density on optimal stem allocation, which indicates selection for a high density phenotype. Second, I discuss the role and viability of tolerance as an alternative to plant defence, which highlights the importance of modelling form and function when studying growth-defence integration. Third, I discuss the importance of considering herbivore community dynamics when studying growth-defence integration. To illustrate this importance I present additional simulations that show how specialist herbivores select against taxonspecific forms of defence expression, while generalist herbivores select for overexpression of taxon-specific forms of defence. Fourth, I discuss how considering multiple resources may change the optimal balance between the acquisition and protection of resources. Finally, I present my view on the future perspectives for FSP models of growth and defence, discussing the integration of multiple resources, neighbour detection cues, and dynamics resulting from a complex representation of plant and herbivore communities. 



\section{Nederlandse samenvatting}

Om te kunnen groeien, heeft een plant licht, water en voedingstoffen nodig. Licht wordt ingevangen met de bladeren, terwijl water en voedingsstoffen uit de grond worden opgenomen door de wortels van de plant. Deze drie componenten worden vervolgens gebruikt om door middel van fotosynthese $\mathrm{CO}_{2}$ om te zetten in suikers, de bouwstof voor de plant. Planten komen zelden alleen voor en zullen daarom de concurrentie aan moeten met buurplanten voor het verkrijgen van licht, water en voedingsstoffen. Naast hun buurplanten hebben planten ook te maken met organismen die de plant gebruiken als voeding, zoals plantenetende insecten. Als de bladeren van een plant worden aangevreten door deze insecten is dat schadelijk voor de plant, omdat hij niet alleen de energie en voedingsstoffen verliest die hij in die bladeren heeft geïnvesteerd, maar ook omdat hij minder licht kan invangen door het verlies van bladoppervlak en daardoor minder kan groeien. Om hun bladeren tegen plantenetende insecten te verdedigen maken planten giftige stoffen aan. Het aanmaken van deze stoffen kost echter energie en voedingsstoffen die ook gebruikt hadden kunnen worden om te groeien en mogelijk de concurrentieslag te winnen van de buurplanten. Planten moeten dus een balans zien te vinden tussen het investeren van kostbare energie en voedingsstoffen ofwel in groei, ofwel in verdediging. De optimale balans tussen deze twee mechanismen hangt af van de omgeving waarin de plant zich bevindt, welke nooit constant, maar altijd in verandering is. Planten kunnen dan ook reageren op de aanwezigheid van buurplanten en plantenetende insecten met het stimuleren of afremmen van groei en verdediging. Om te kunnen reageren op veranderingen in de omgeving, gebruikt de plant verschillende signalen. Doordat bladeren rood licht absorberen en ver-rood licht reflecteren of doorlaten, is de ratio rood tot ver-rood licht in het spectrum (R:FR) een belangrijk signaal waarmee de aanwezigheid van buurplanten kan worden gedetecteerd. Dit lichtsignaal stimuleert groeipatronen die nodig zijn om de concurrentie om licht met de buurplanten te winnen. Tegelijkertijd verlaagt het de verdediging van de plant, waardoor hij kwetsbaarder wordt voor schade door plantenetende insecten. Men denkt dat dit mechanisme een manier is om de optimale balans te vinden tussen investeren in groei en verdediging, waarbij het winnen van de concurrentie om licht belangrijker is dan het verdedigen tegen plantenetende insecten. Het lastige is echter dat de optimale balans tussen groei en verdediging afhangt van interacties met de aanwezige plant- en insectengemeenschappen en daarom zeer complex is. Het doel van dit proefschrift is om uit te zoeken hoe deze ecologische interacties de optimale balans tussen competitieve groei en verdediging beïnvloeden. 
Om dit doel te bereiken maak ik gebruik van driedimensionale computermodellen die expliciet de architectuur van de plant simuleren en dat koppelen aan een mechanistische beschrijving van functionele processen zoals fotosynthese en verdediging tegen insecten. Deze modellen zijn uitermate geschikt om de interactie tussen de plant en zijn omgeving te simuleren, waarbij de interacties tussen planten ontstaan uit de mechanismen die in het model zijn beschreven. Wanneer bijvoorbeeld een blad wordt aangevreten door een insect vermindert dit het bladoppervlak van de plant en daarmee het vermogen van de plant om licht in te vangen. Als gevolg daarvan zal de plant minder kunnen groeien. Daarnaast zal een buurplant juist meer kunnen groeien, door het licht in te vangen wat eerst door het aangevreten blad werd ingevangen. Met deze modellen kan ik het effect van één enkele verandering in de structuur of functionaliteit van een plant doorrekenen in verschillende condities, bijvoorbeeld hoe een grotere investering in afweer de prestaties van de plant beïnvloedt bij een hoge populatiedichtheid. Door deze modellen te koppelen met een evolutionair algoritme kan ik nog een stap verder gaan en doorrekenen wat de optimale combinatie van eigenschappen is onder een bepaalde combinatie van condities.

In deze thesis laat ik zien dat de dynamische interacties met de omgeving en de organismen in die omgeving zeer bepalend zijn voor de optimale balans van groei en verdediging en dat deze leidend zijn geweest in de evolutie van planten. Ik laat zien dat de identiteit van de insecten die de plant aanvallen, de aanwezigheid en strategie van de buurplanten en de beschikbaarheid van voedingsstoffen in de bodem allemaal van invloed zijn op de optimale balans tussen groei en verdediging. Ook laat ik zien dat competitie tussen planten ervoor zorgt dat planten zich niet optimaal verdedigen tegen vraat en dat de rol van R:FR signalering alleen niet voldoende is om een optimale investering van afweer bij verschillende populatiedichtheden de realiseren. In dit proefschrift demonstreer ik dat driedimensionale computermodellen voldoende zijn doorontwikkeld om de complexe interacties tussen de plant en zijn omgeving te kunnen bestuderen. Ik denk dat toekomstige ontwikkelingen in driedimensionale computermodellen verdere inzichten zullen opleveren in de werking van groei en verdediging en dat deze methodiek onmisbaar zal worden voor toekomstig experimenteel onderzoek, de veredeling van gewassen en het beheren van landbouwsystemen. 


\section{Acknowledgements}

I want to start by thanking my supervision team. Jochem, thank you for giving me the freedom to carve my own path in both my MSc and $\mathrm{PhD}$ projects, yet providing just the right amount of guidance to keep me on track. I am grateful that you drove me back and forth to Wageningen when I was injured, and that you allowed me to barge in with a quick question at any time. Niels, I am glad that you introduced me to modelling during my first MSc project, and grateful that you brought me to Wageningen for a second MSc project and later for my $\mathrm{PhD}$. Your insights into a game-theoretical way of thinking has been instrumental to this thesis, and your influence is very clear in its final two chapters. Erik, thank you for your kindness, hospitality and your insights in a world of complex interactions, and thank you for allowing me the freedom to reduce your beloved insects to the simplest of mathematical equations. Marcel, thank you for looking after me even though you saw very little of me, and thank you for your critical comments on my manuscripts, they really made a difference.

Although I owe much to all of my colleagues, both in the Centre for Crop Systems Analysis and Entomology, I want to mention a few colleagues to whom I have been especially close. Bob, I have really enjoyed our collaboration, while it did take some effort to combine our different viewpoints and modus operandi, you have taught me a great deal about myself and the way I do research. I'm very proud that our efforts resulted in the fifth chapter of this thesis and a swift publication in an excellent journal, let's do that again sometime. Franca, I deeply miss our heated discussions at the Plant Form and Function meetings, to the point that others thought we hated each other. That still makes me smile. Thank you for asking me to stand by your side as you defended your thesis, I very much enjoyed it. Rachel, it has been great fun to go from your MSc supervisor to co-worker to friend, and I really enjoyed us organising the PFF meetings together. Quint, thank you for all the laughs we've had, I especially enjoyed our time at the Gordon conference, surfing in the Pacific was awesome. I am very grateful to Rachel and Quint for agreeing to be at my side when I defend this thesis.

I want to thank Junqui, Marcello, Alejandro, Martin, Maarten, Nighyi, Arjen, Ioannis, Gu, Ambra and all of the other MSc's and PhD's (and the occasional guest) who participated in the fruitful discussions at the PFF meetings, your insights have been instrumental in the development of my FSP models and of me as a researcher. I also want to thank Erik's team, Marjolein, Feng, Jeltje, Yavanna, Quint, Martin, Daan, Maite, Janneke, Ilka and Klaas, thank 
you all for helping to gain a better understanding of the wonderfully complex world of insects. I want to thank everyone who helped out in the field, the Ento colleagues who rushed in to help with planting, Niewold and Bob who offered their assistance on a couple of measurement days, the Unifarm crew who kept my fields free of bugs and weeds, and the insect rearing team who lovingly raised the insects used in the experiments. Ambra, Luuk and Martin, thanks for bringing the fun into our little fish tank. TJ, thanks for all the interesting brainstorms, and Henny, bedankt voor het lachen!

Diep, Yi, Martina, Jos, Jojanneke and Lingli, thanks for wanting to do your MSc thesis with me, you have taught me a great deal and have provided me with valuable insights through your experimental work. A special note to Jos, whose dedication and persistence I admire greatly, and to Martina, whose kindness struck me.

Liesje and Jasper, I have always enjoyed our meetings trying to keep the root modelling work rolling, and I am super exited that we finally have the opportunity to bring our collaboration into a publication. Liesje and Jochem, your pitch performance was awesome!

And finally, I want to thank Lydian for being with me throughout this journey to share both the challenges and the successes. I hope we get to share a lot more of the latter in the coming years. I am happy that I get to go adventuring with you. 


\section{Curriculum Vitae}

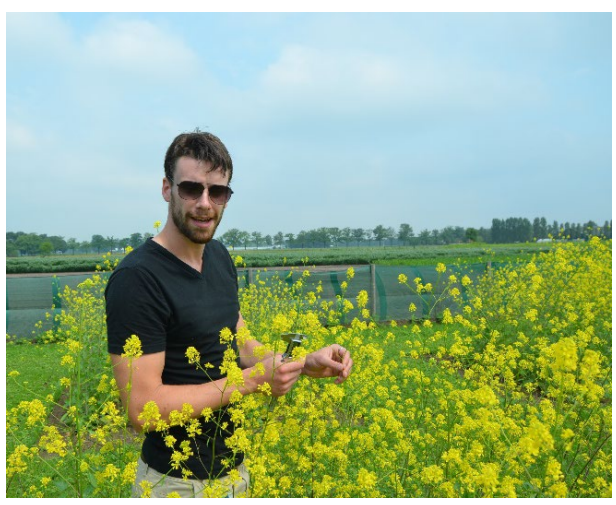

Jorad de Vries was born on December $7^{\text {th }}, 1986$ in Breda, The Netherlands. After finishing high school in Den Haag, he moved to Utrecht to study Biology at Utrecht University. After finishing his bachelor Biology with a focus on ecology, he started the master Environmental Biology at Utrecht University. During the first of two research internships he came into contact with plant growth models and conducted a theoretical study on forest productivity under the supervision of Dr. Niels P.R. Anten. For the second research internship, he went to Wageningen University and developed a functionalstructural root growth model under the supervision of Dr. Jochem B. Evers. In 2013, he started his $\mathrm{PhD}$ research at the laboratory of Entomology and Centre for Crop Systems Analysis of Wageningen University under the supervision of Prof. Dr Marcel Dicke, Prof. Dr Niels P.R. Anten, Dr. Erik H. Poelman and Dr. Jochem B. Evers. In his PhD project he studied how plants balance their investment in competitive growth and defence against herbivorous insects, using a functional-structural plant modelling approach. In the development of these models he combined field experiments with physiological, ecological and evolutionary principles. In December of 2018 he started a 4-month post-doctoral project to expand and publish the root modelling work he started in his second MSc research internship, in collaboration with Jochem Evers and Dr. Liesje Mommer of the Plant Ecology and Nature Conservation group. In March 2019, Jorad will move to Zürich to continue his scientific career. 



\section{Publications}

de Vries J, Evers JB, Poelman EH. 2017. Dynamic plant-plant-herbivore interactions govern plant growth-defence integration. Trends in Plant Science 22(4): 329-337.

de Vries J, Poelman EH, Anten NP, Evers JB. 2018a. Elucidating the interaction between light competition and herbivore feeding patterns using functional-structural plant modelling. Annals of Botany 121(5): 1019-1031.

de Vries J, Evers JB, Dicke M, Poelman EH. 2018b. Ecological interactions shape the adaptive value of plant defence: herbivore attack versus competition for light. Functional Ecology: 1-10.

Douma JC, de Vries J, Poelman EH, Dicke M, Anten NPR, Evers JB. 2019. Ecological significance of light quality in optimising plant defence. Plant Cell and Environment: in press. 



\section{PE\&RC Training and Education Statement}

With the training and education activities listed below the $\mathrm{PhD}$ candidate has complied with the requirements set by the C.T. de Wit Graduate School for Production Ecology and Resource Conservation (PE\&RC) which comprises of a minimum total of 32 ECTS (=22 weeks of activities)

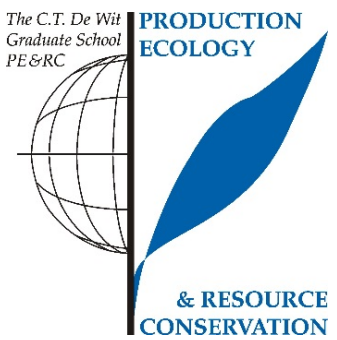

\section{Review of literature (6 ECTS)}

- Dynamic plant-plant-herbivore interactions govern growth-defence integration (2017)

\section{Post-graduate courses (3 ECTS)}

- Introduction to R for statistical analysis; PE\&RC (2014)

- FSPM Summer school; Angers (2015)

- FSPM Summer school; Wageningen (2016)

Invited review of (unpublished) journal manuscript (2 ECTS)

- Annals of applied biology: root architecture and competition (2016)

- Functional ecology: the role of volatiles in plant host finding (2016)

\section{Competence strengthening / skills courses (1.8 ECTS)}

- Scientific writing; Library/WGS (2015)

\section{PE\&RC Annual meetings, seminars and the PE\&RC weekend (1.2 ECTS)}

- Symposium on plant-insect interactions $(2014,2017)$

- Netherlands annual ecology meeting (2015)

\section{Discussion groups / local seminars / other scientific meetings (7.5 ECTS)}

- Plant insect interactions discussion group (2014-2018)

- Plant form and function meeting (2015-2018)

International symposia, workshops and conferences (6.9 ECTS)

- iCROP; Berlin (2016)

- FSPMA; Quingdao (2016)

- Gordon conference on plant herbivore interactions; Ventura CA (2017)

\section{Lecturing / Supervision of practicals / tutorials (13.5 ECTS)}

- Ecological aspects of bio-interactions (2014-2017)

- Functional diversity (2014-2018)

\section{Supervision of MSc students}

- Jos Paul: effect of plant competition and herbivory by Pieris brassicae on photosynthesis and defence response of Brassica nigra

- Jojanneke Voorhoeven: leaf tip of Brassica nigra is most sensitive to low R:FR ratio's

- Rachel Schipper: nitrogen driven plasticity in the root system architecture of Anthoxanthum odoratum 
The research described in this thesis was financially supported by the Netherlands

Organisation for Scientific Research (NWO - ALW Open competition grant 823.01.004 to E.H.P)

Layout and cover design by the author

Printed by GVO drukkers \& vormgevers, Ede, the Netherlands 


\section{Propositions}

1. Plant growth-defence integration is an arms race on multiple fronts, and should be considered in the context of evolutionary game theory.

(this thesis)

2. The adaptive value of a plant strategy cannot be assessed in a single ecological setting, but should be considered in the context of long-term variation in biotic and abiotic factors.

(this thesis)

3. The concepts of natural selection and game theory are imperative to tackle global problems such as poverty and climate change.

4. $\mathrm{PhD}$ projects should be designed around collaboration rather than standing alone to improve their efficiency and scientific impact.

5. Chair groups should employ more managers and technical staff to allow the brightest and most experienced researchers to contribute more to science.

6. Science should stimulate the publication of results that support null-hypotheses, to reduce conformation bias and increase the efficiency of science as a whole.

7. People in knowledge-based occupations, such as in science, should work fewer hours to increase both creativity and productivity.

8. Misinformation of the public by politicians gives them an unfair advantage during elections that is akin to doping in sports and should be punished as such.

Propositions belonging to the $\mathrm{PhD}$ thesis entitled:

"How plants balance competitive growth and defence: an analysis of virtual plants in dynamic interactions"

Jorad de Vries

8 February 2019 


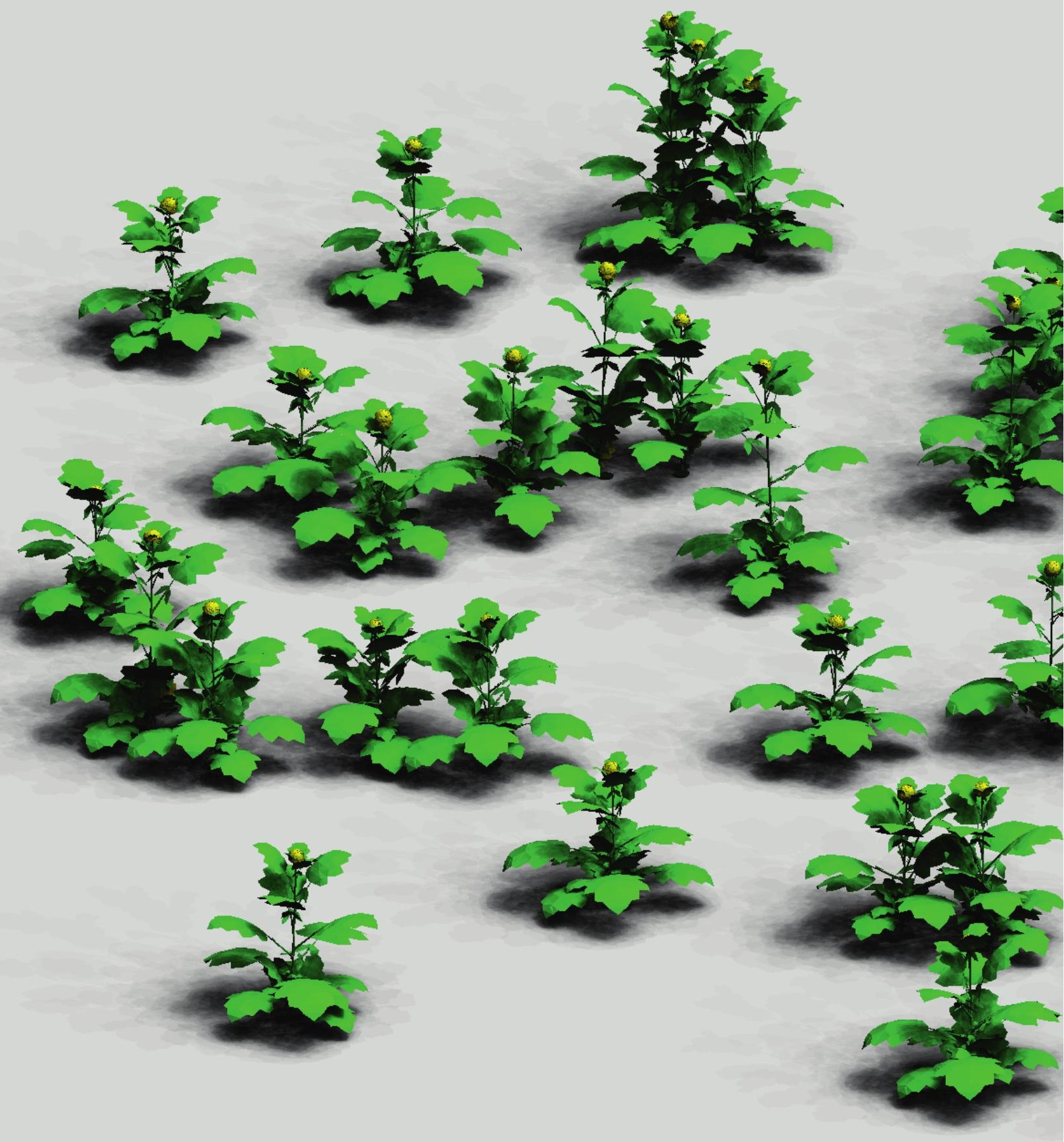

